Fall 1959

\title{
1959 Miracle Yearbook
}

\section{Cedarville College}

Follow this and additional works at: https://digitalcommons.cedarville.edu/yearbooks

Part of the Higher Education Commons, Organizational Communication Commons, and the Public Relations and Advertising Commons

\section{Recommended Citation}

Cedarville College, "1959 Miracle Yearbook" (1959). Yearbooks. 40.

https://digitalcommons.cedarville.edu/yearbooks/40

This Book is brought to you for free and open access by DigitalCommons@Cedarville, a service of the Centennial Library. It has been accepted for inclusion in Yearbooks by an authorized administrator of DigitalCommons@Cedarville. For more information, please contact digitalcommons@cedarville.edu. 

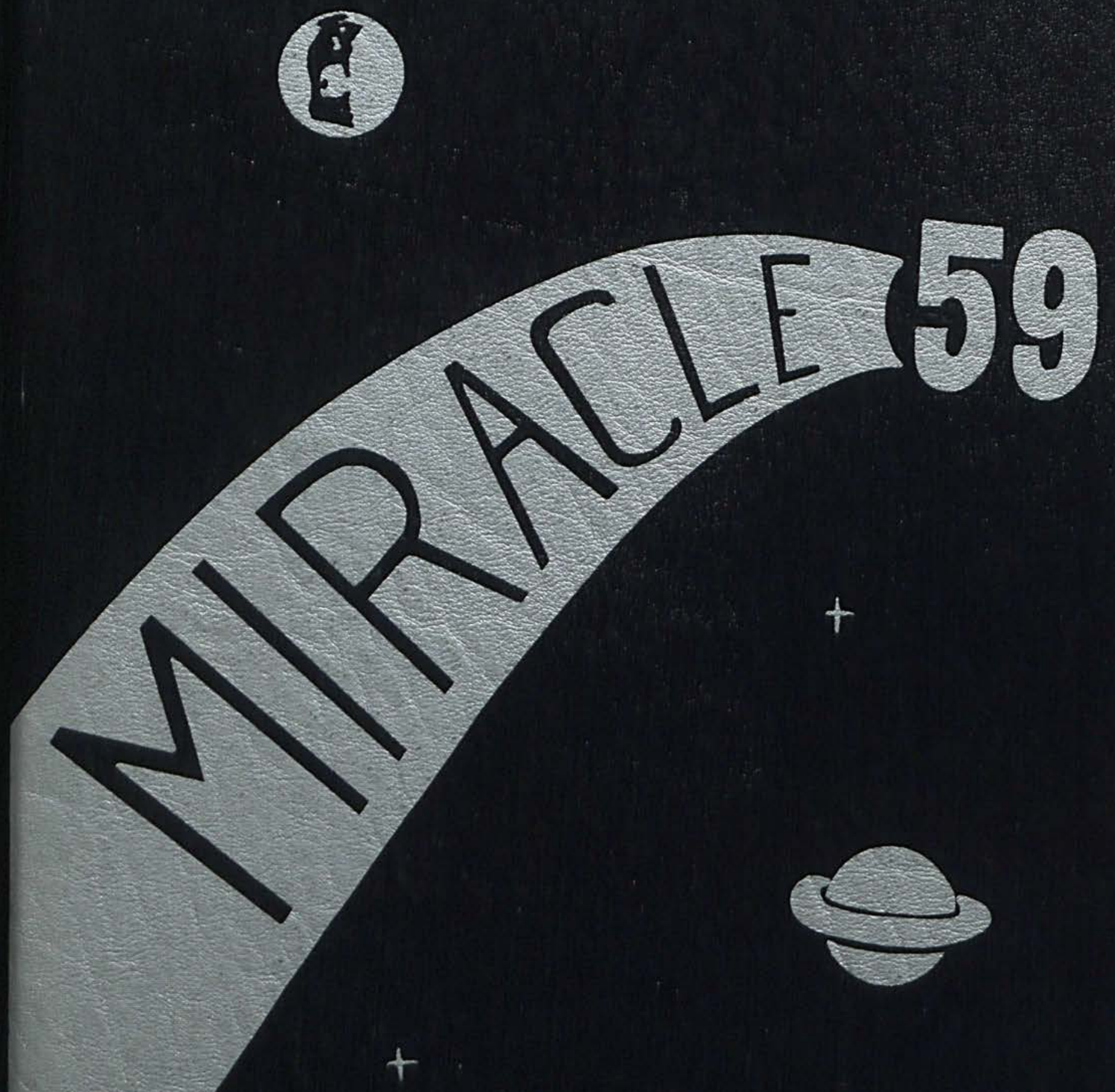


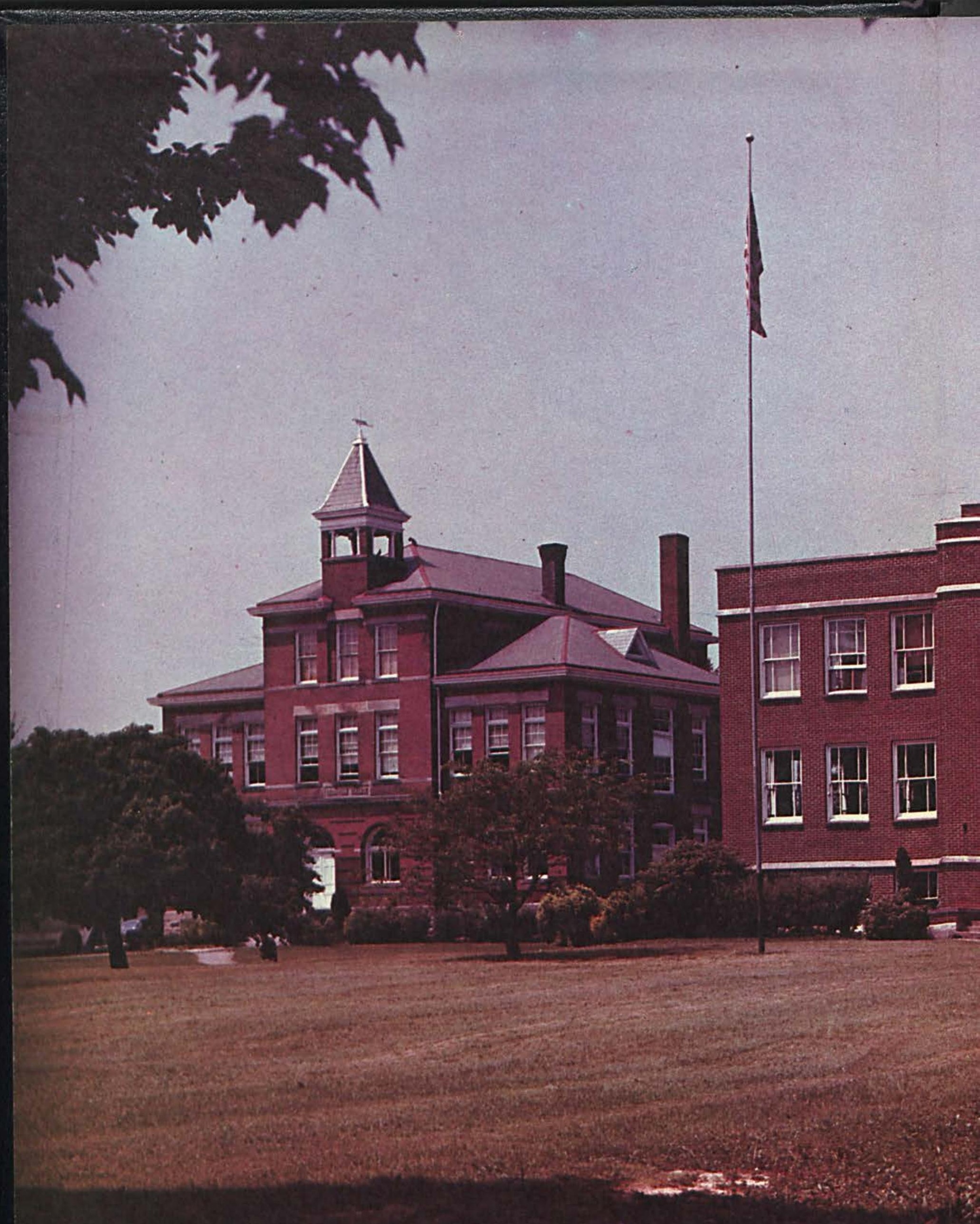




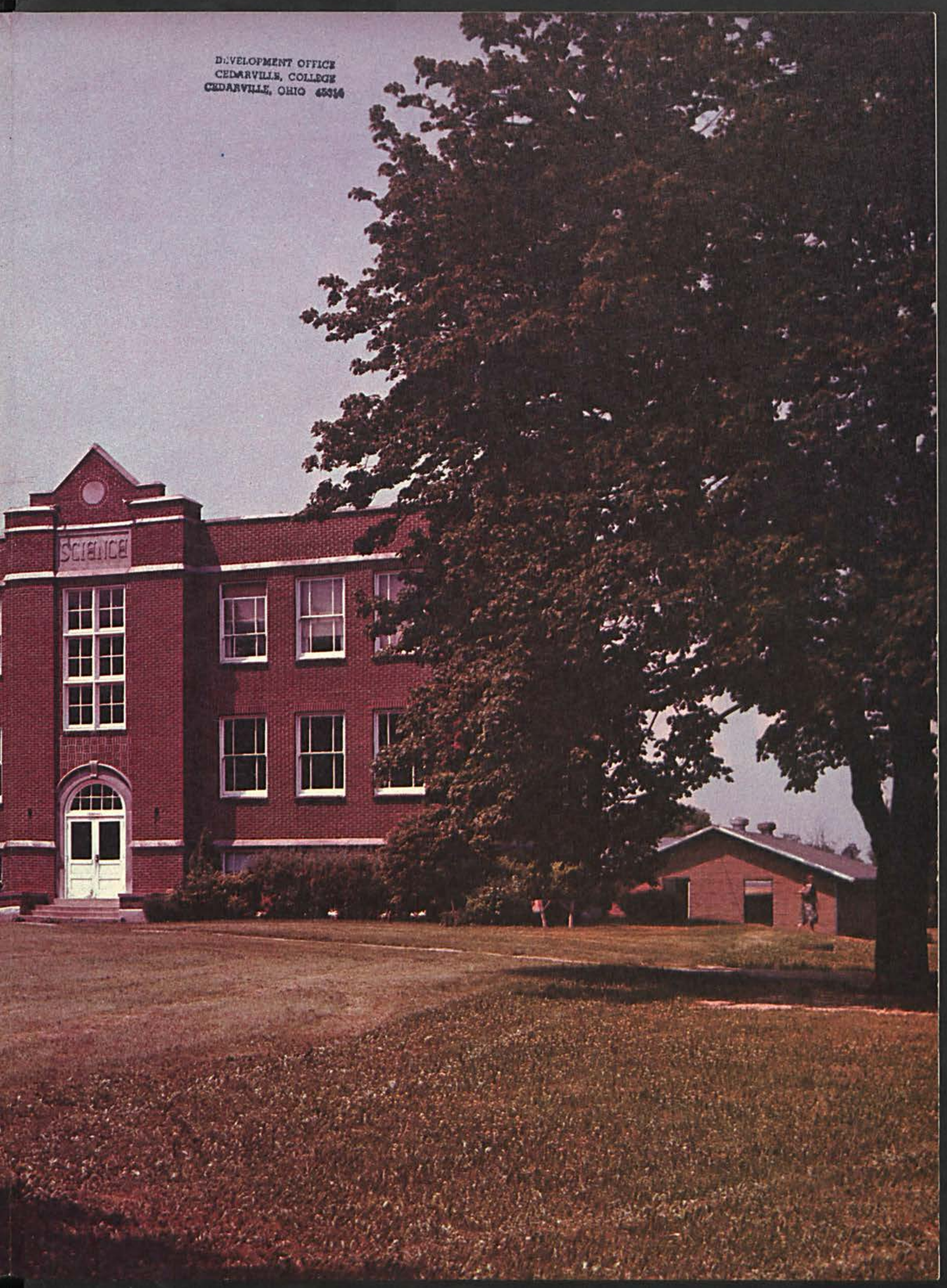




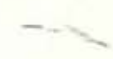




\section{The Miracle}

From early childhood . . . through student days . . . to adult achievement . . . discovery is a continuing process of life through which each must pass. It may be through music or the scientific realm, by the gaining of knowledge and skills in a classroom, by diligent, constant study on our own, or through the guidance and instruction from those who help in molding our lives for the future.

Discovery is not all hard work. Joy, pleasure, and lasting friendships help to make it a pleasant experience, whether through student parties, roommates, or the exercising of our bodies in sports. Yes, life is discovery. Only time, work, and study will reveal the fulfillment of that dream of early childhood as it becomes a reality at Cedarville College. Herein is recorded one year of our great discovery. 


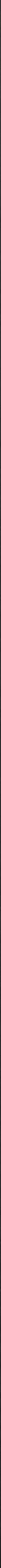





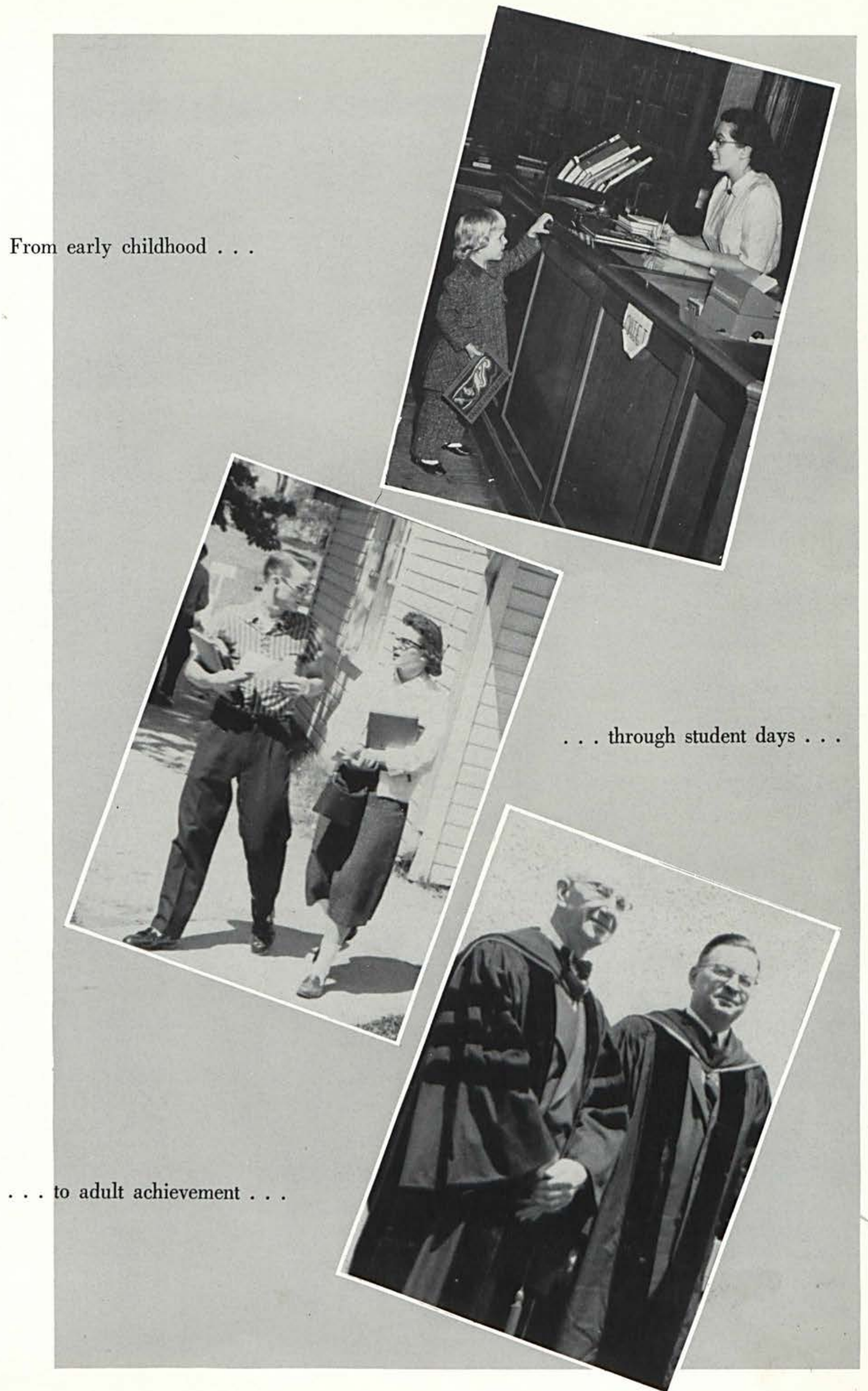




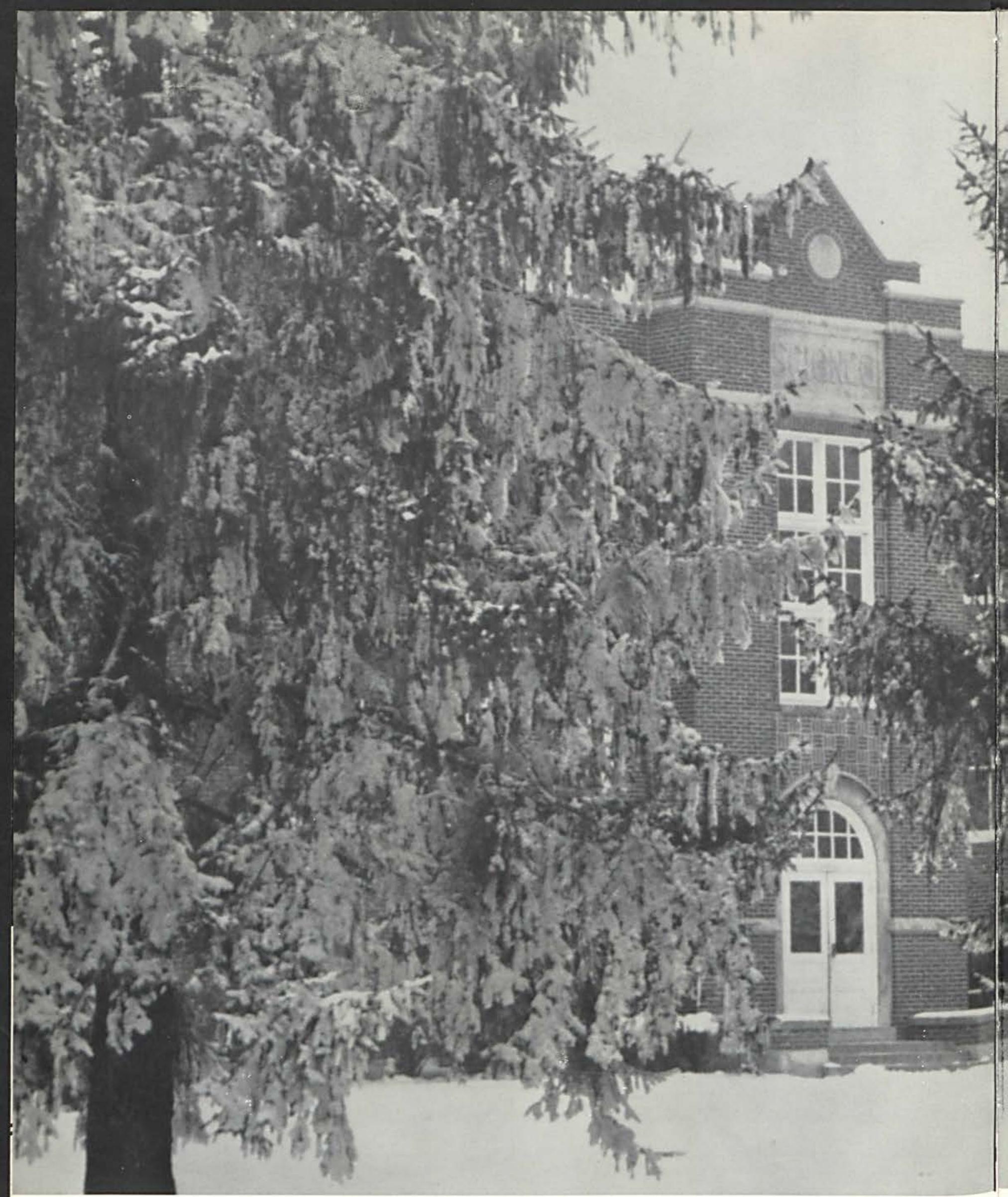

.. discovery is a continuing process of life through which each must pass. It may be through music or the scientific realm . . . 

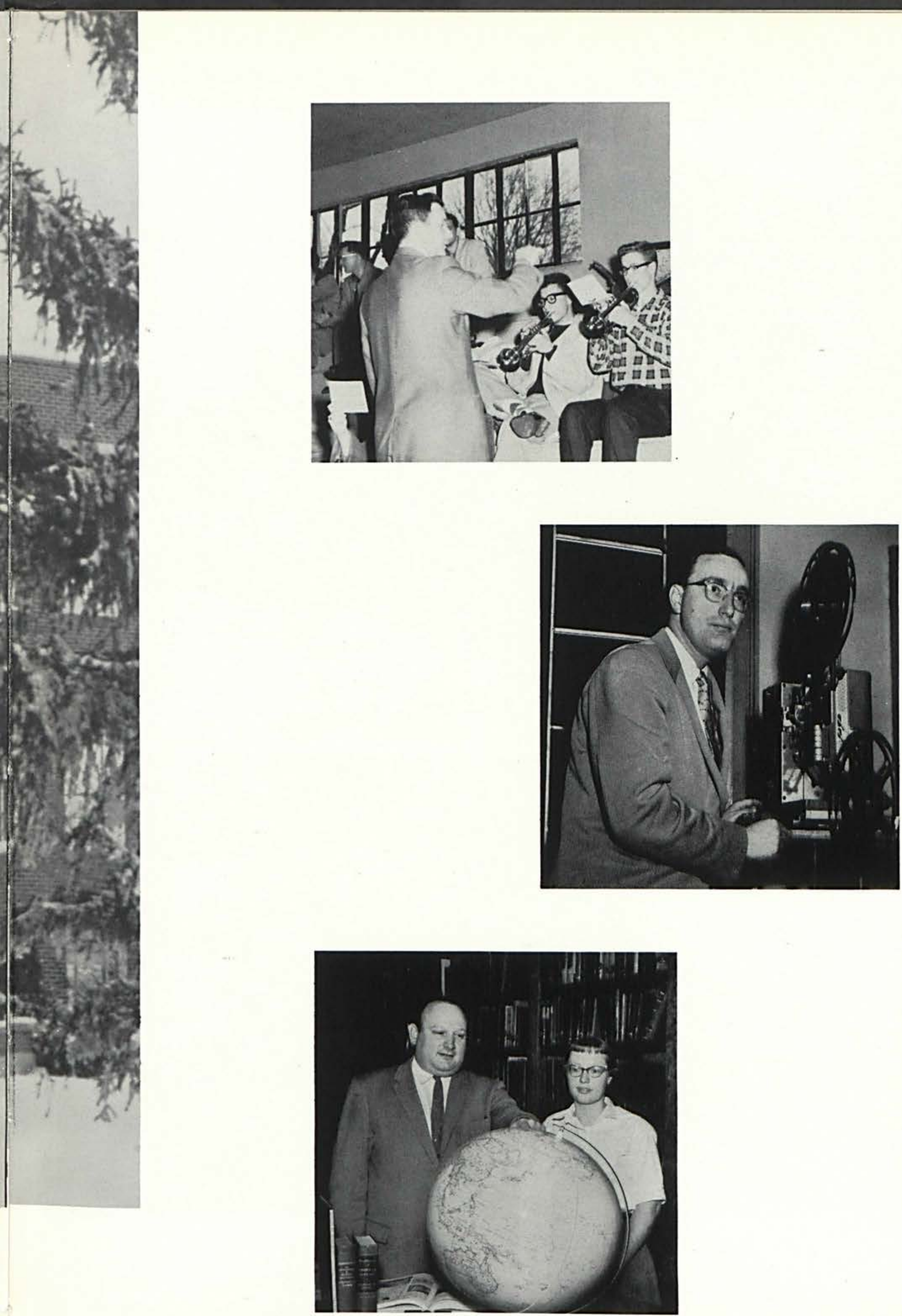

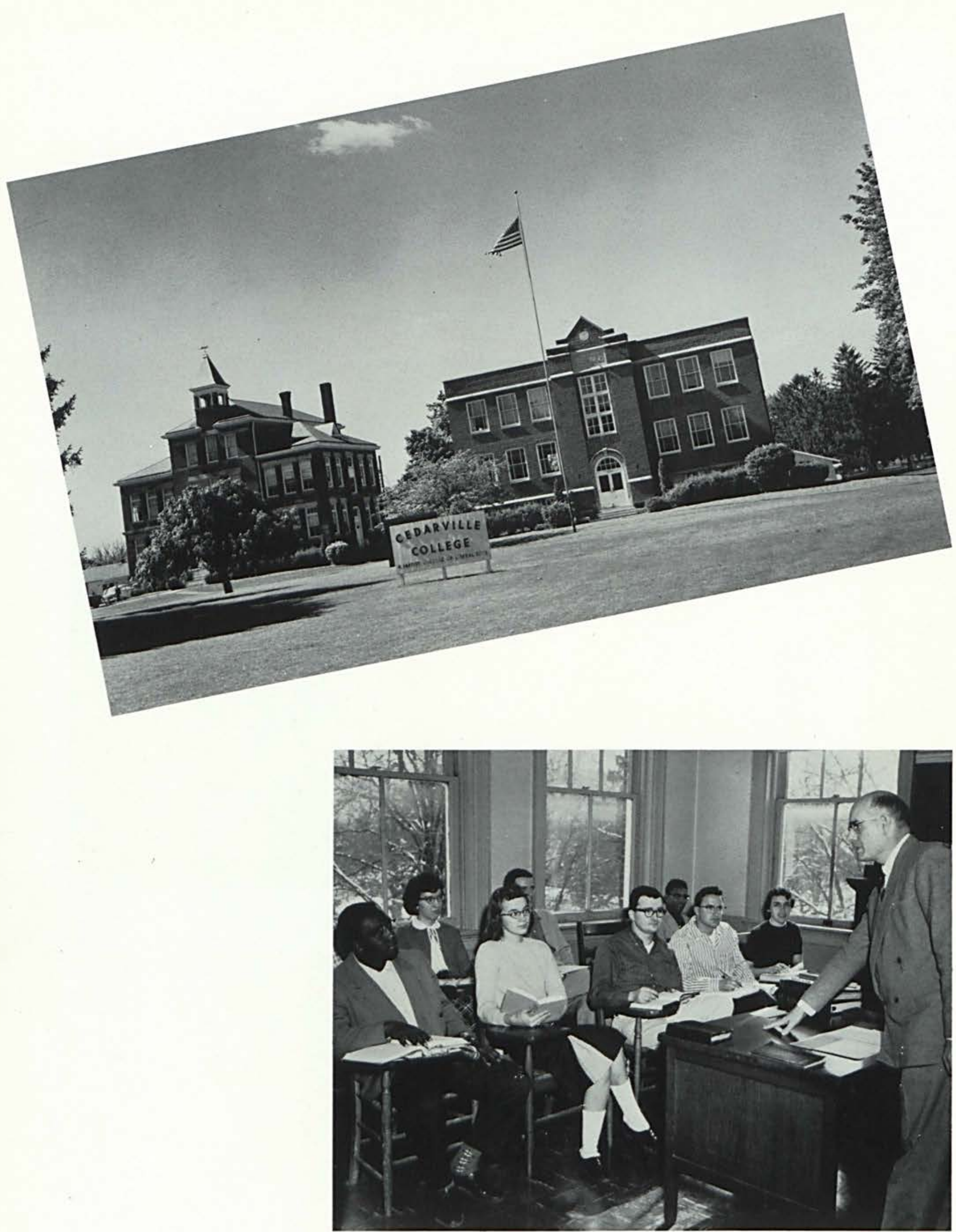

... the gaining of knowledge and skills in a classroom... 


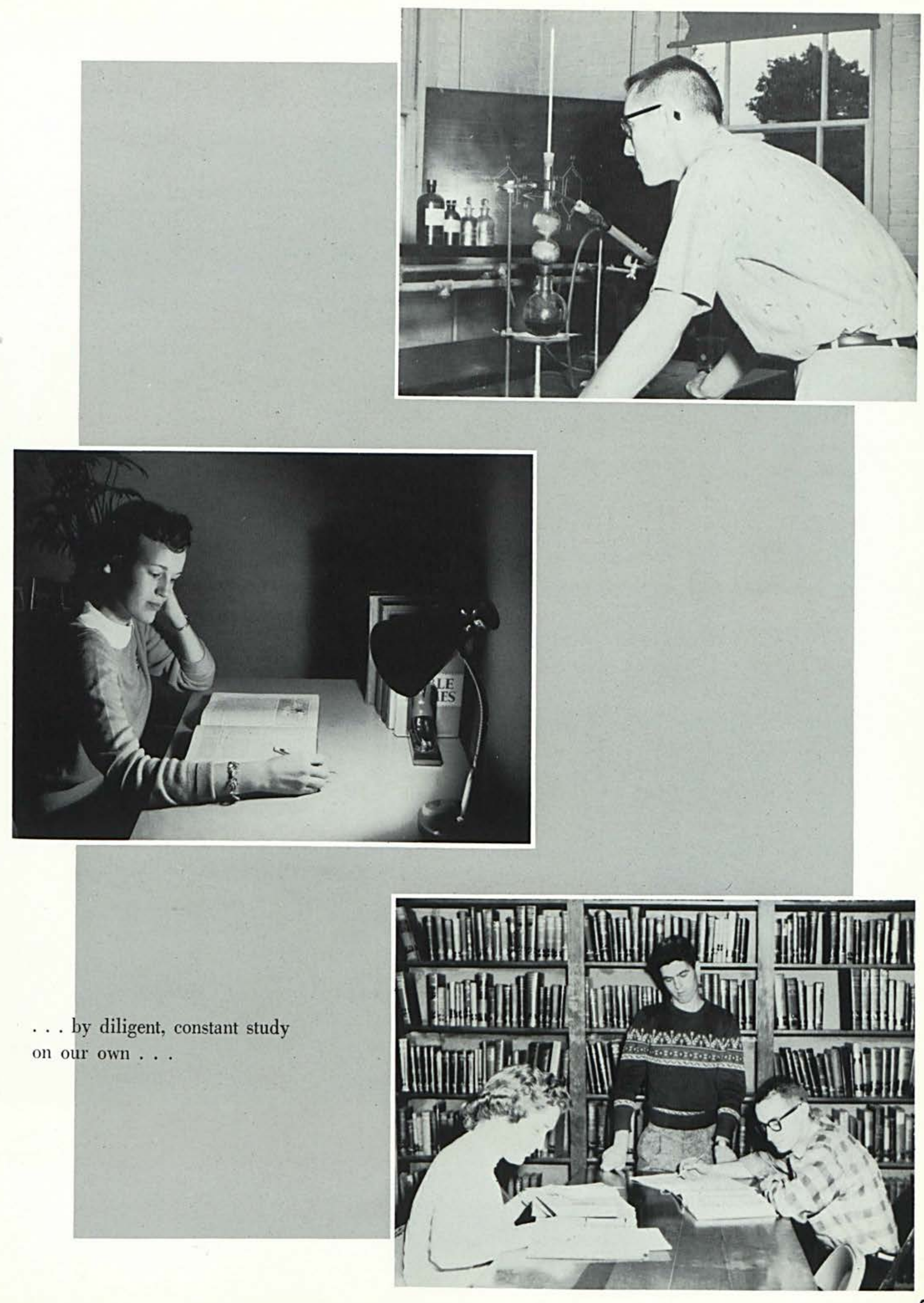




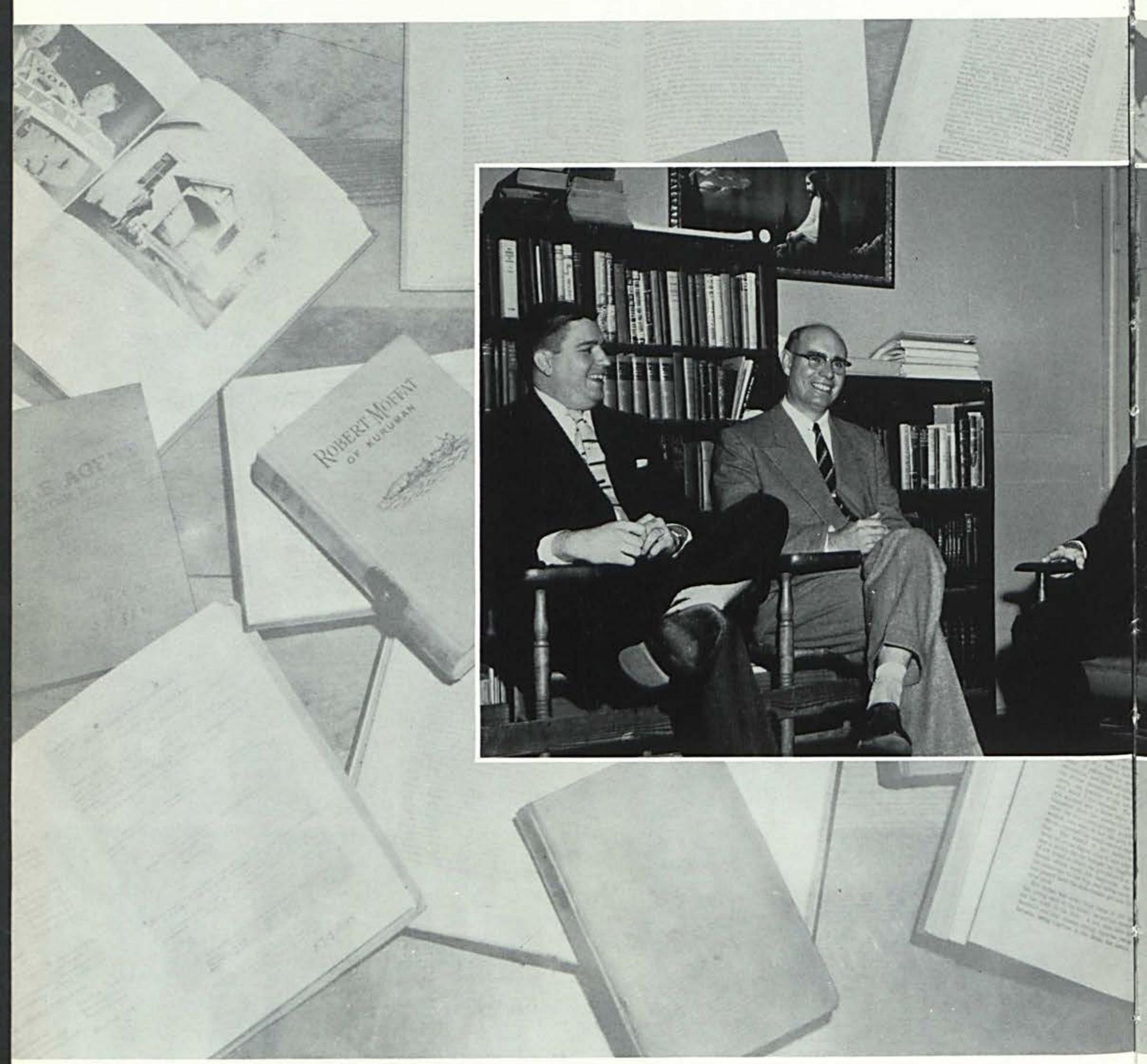

... all guided and instructed by those who help in molding our lives to the future ... these, our professors. 


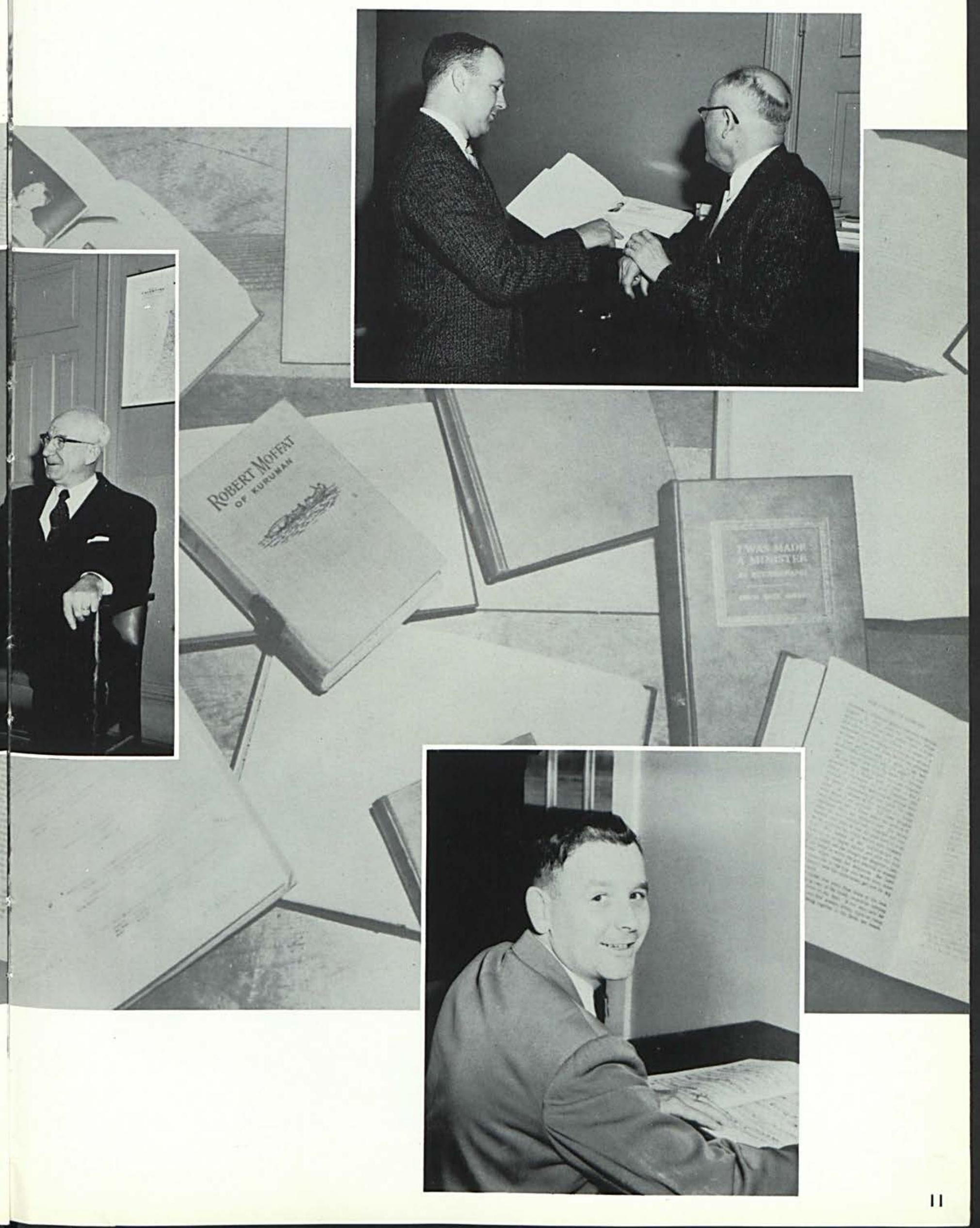


Discovery is not all hard work. Joy, pleasure and lasting friendships help to make a more pleasant experience . . . whether through student parties, roommates or... 


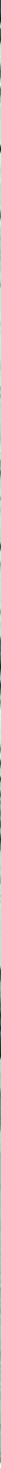

... by the exercising of our bodies in sports. 


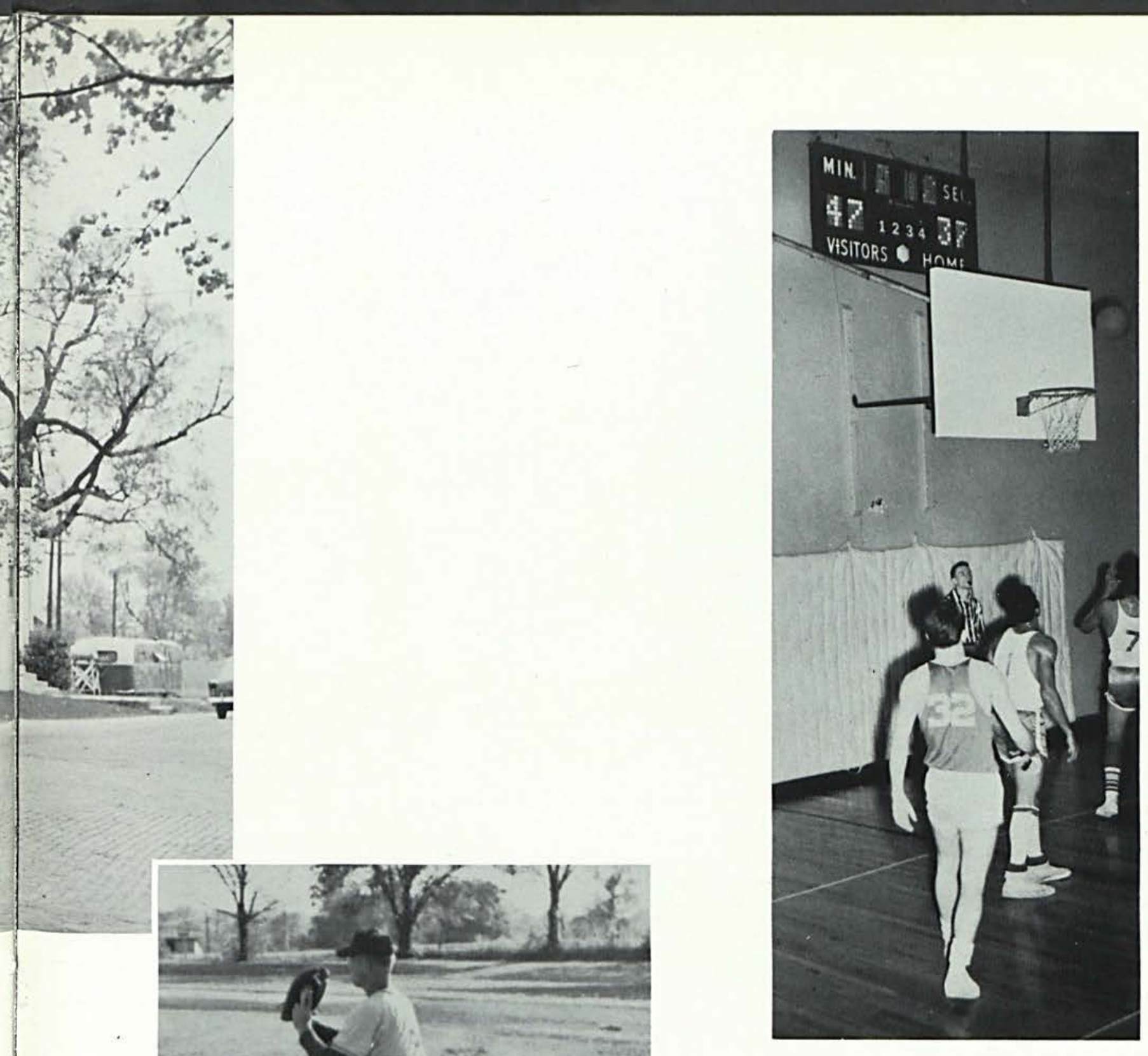




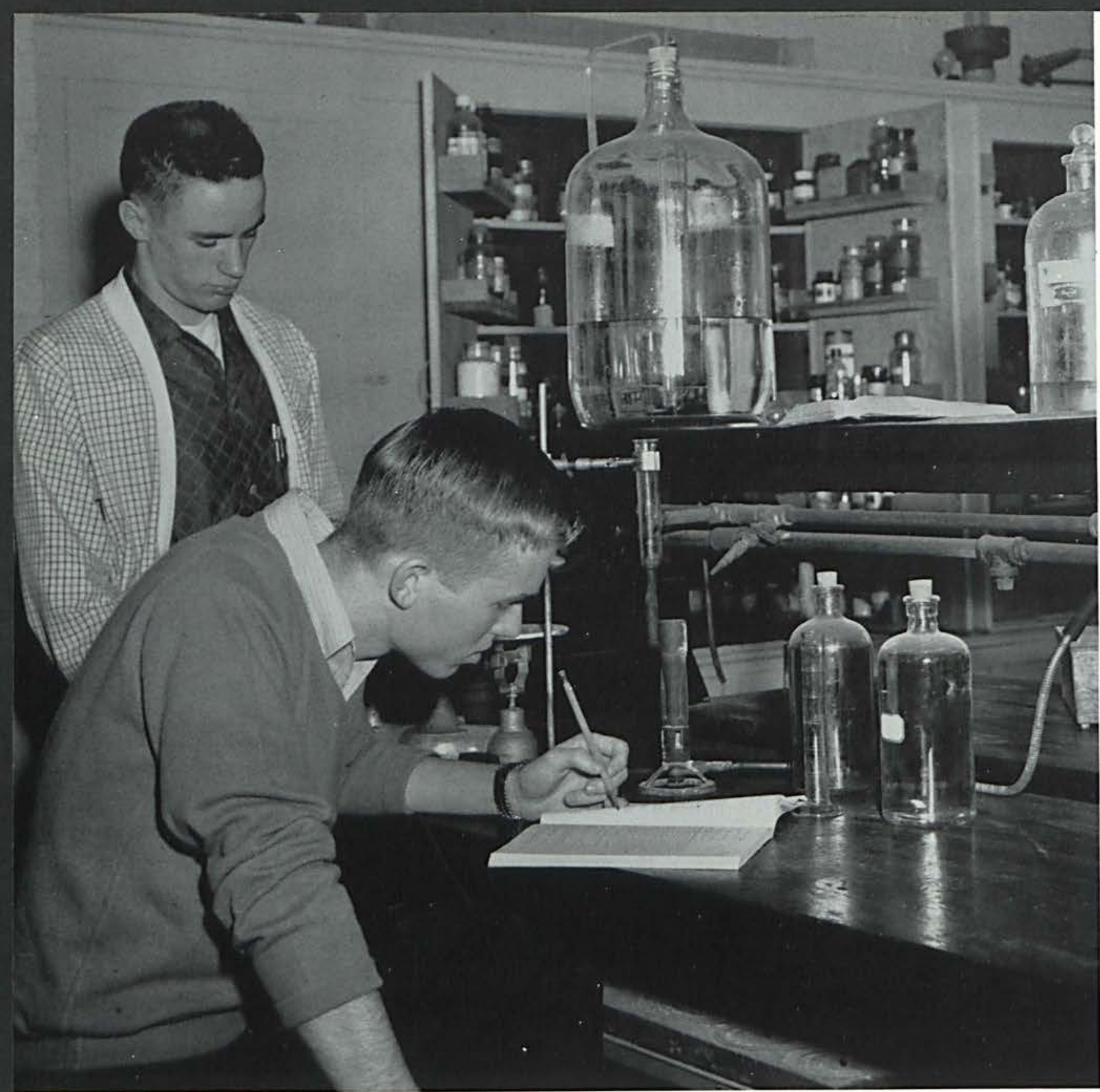

Only time, work, and study will reveal the fulfillment of that dream of early childhood as it becomes reality at Cedarville College . . . our great discovery. 


\section{TABLE OF CONTENTS}

DEDICATION......... laborers together

MASTERS.........faculty

SEARCHERS......... students

DISCOVERIES.......... knowledge

friends

joy

skill

pleasure

truth 


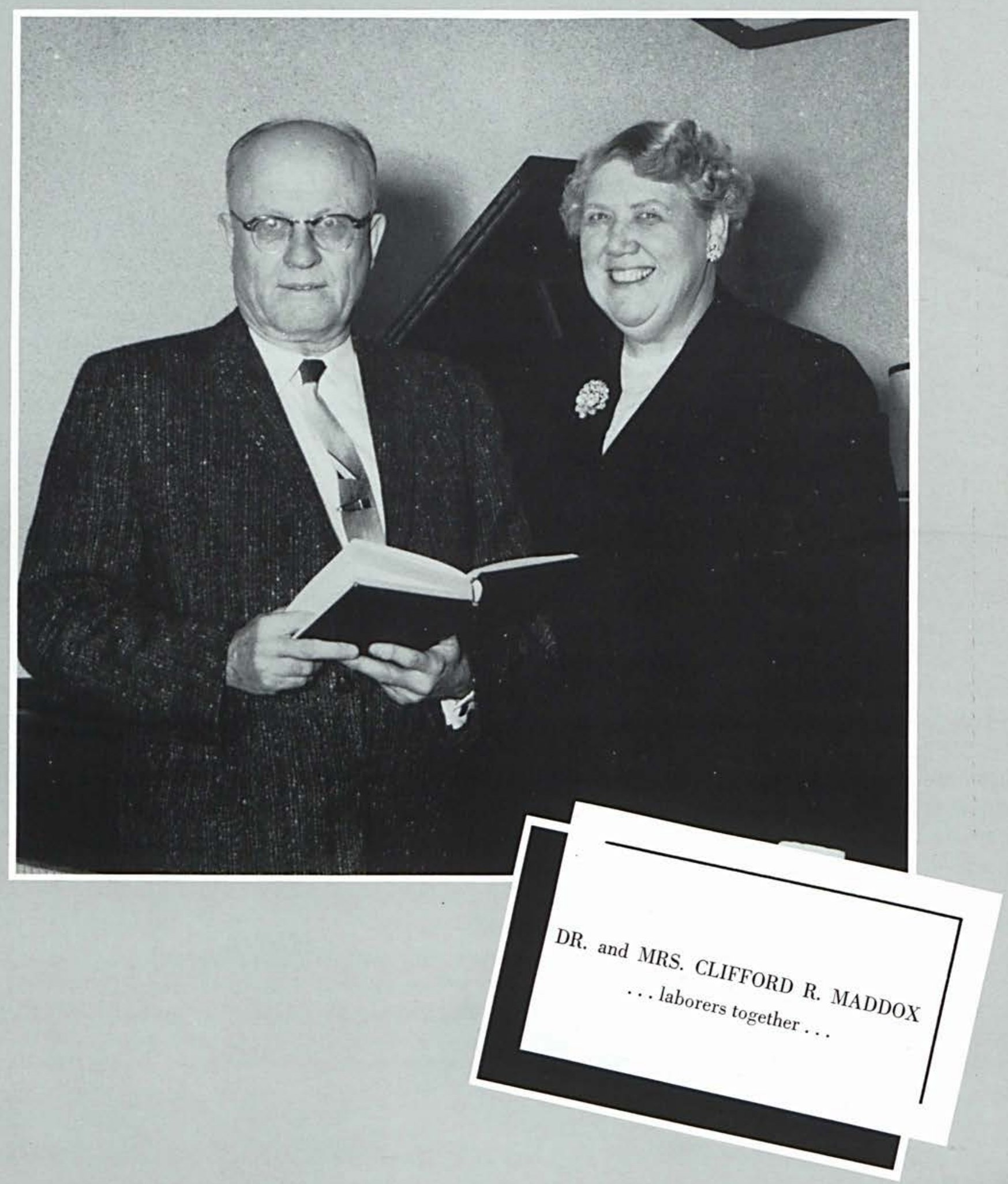




\section{DEDICATION}

Because of your vast contributions to the academic, social, and spiritual welfare of our school, we the Junior Class proudly dedicate our yearbook to you Dr. and Mrs. Clifford R. Maddox.

During your years at Cedarville you have given unselfishly of your time and talents to the betterment of our school. Your untiring enthusiasm and faithful service will long be remembered as contributing factors in our school's history. We cannot even begin in this short space to enumerate your efforts put forth to help Cedarville College grow in the wisdom and knowledge of our Lord.

We, the Junior Class, sincerely thank you and will continue to uphold you in prayer as you labor for Him who loved us and gave Himself for us. 


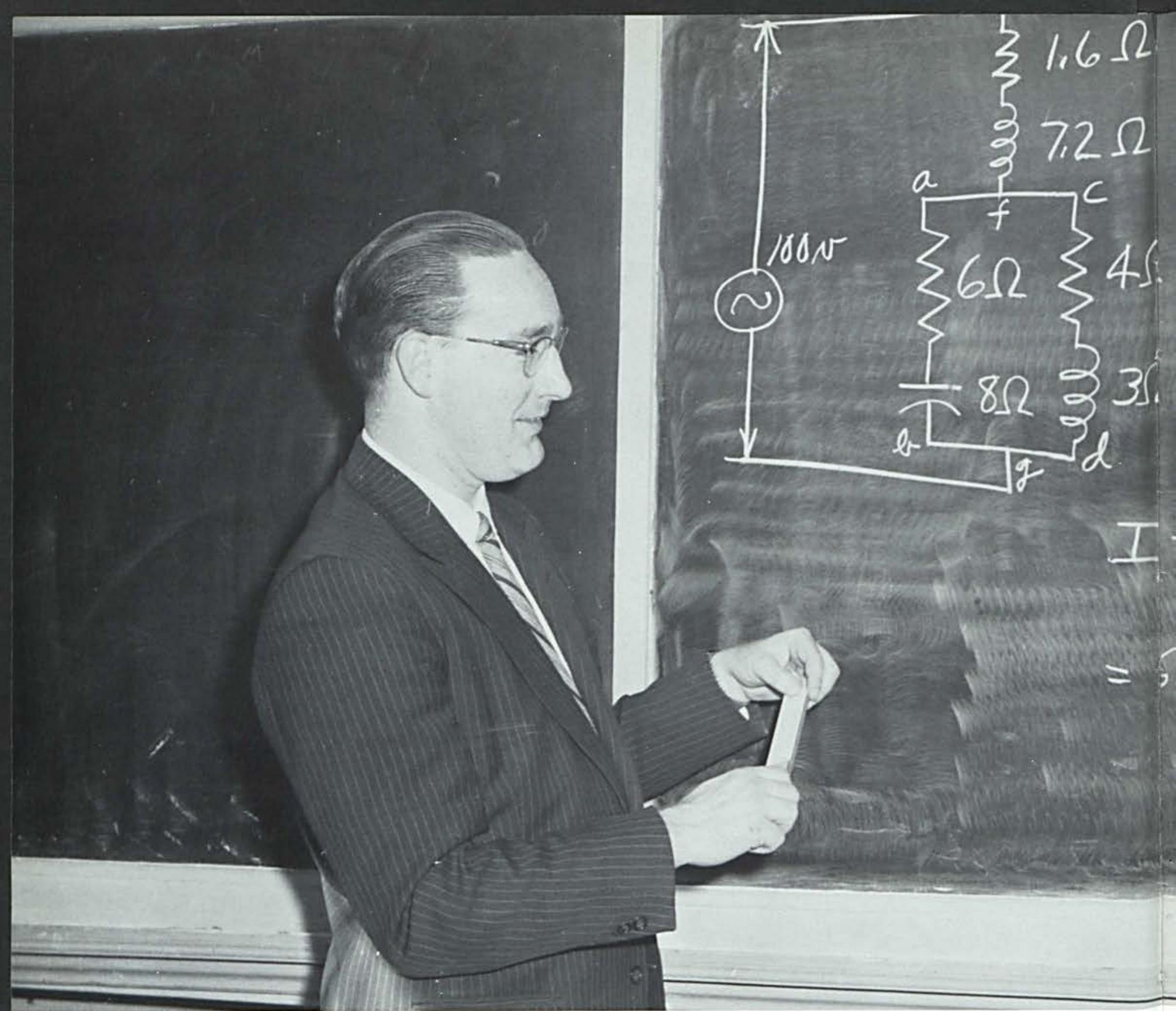




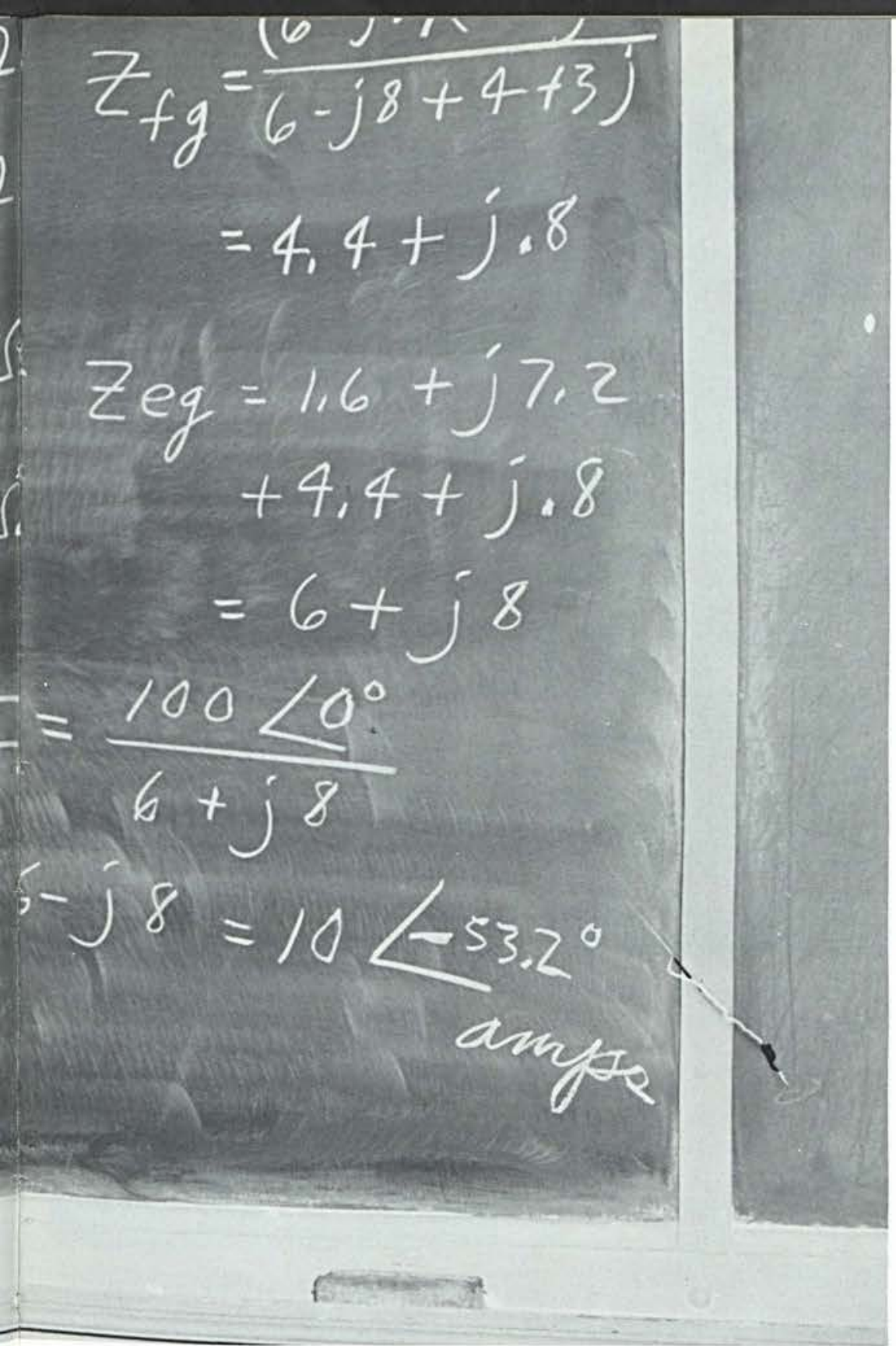




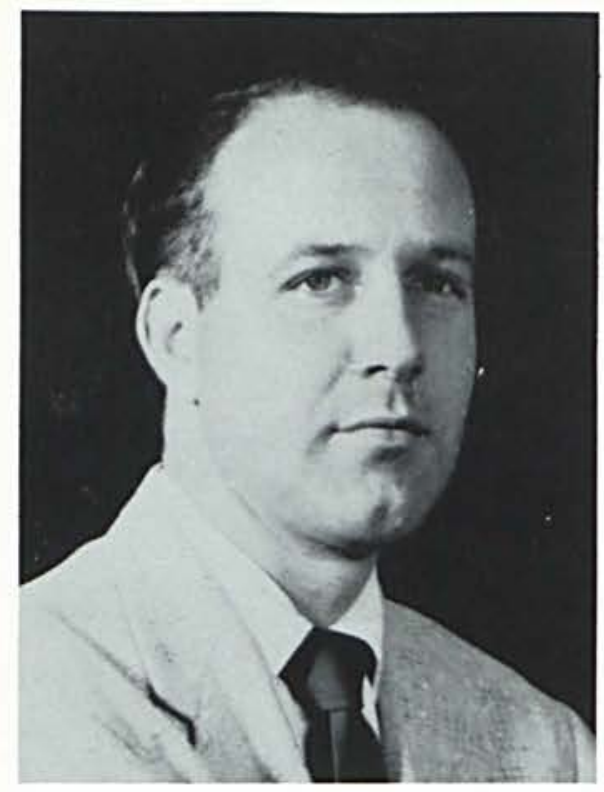

ROBERT UNDERWOOD, M.A.

Registrar

Education

\section{Deans}

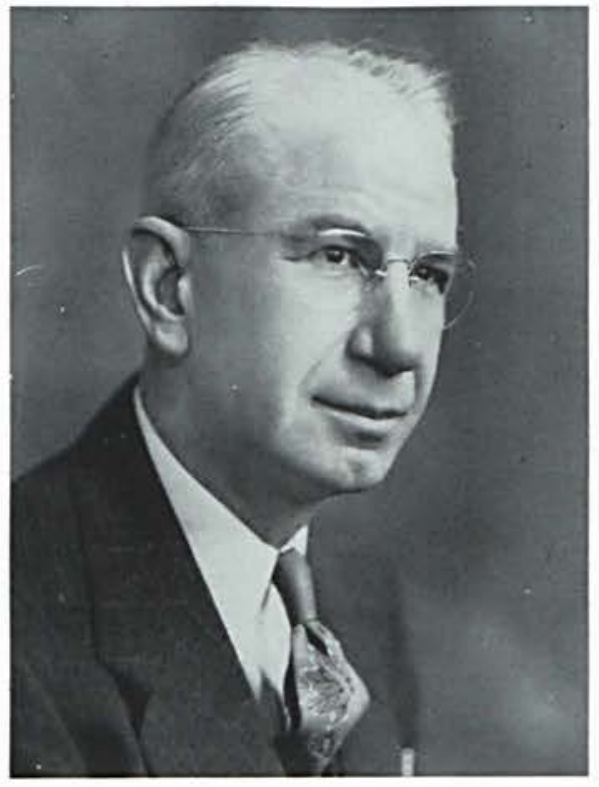

ARTHUR F. WILLIAMS, B.A., D.D. Dean of Students Bible

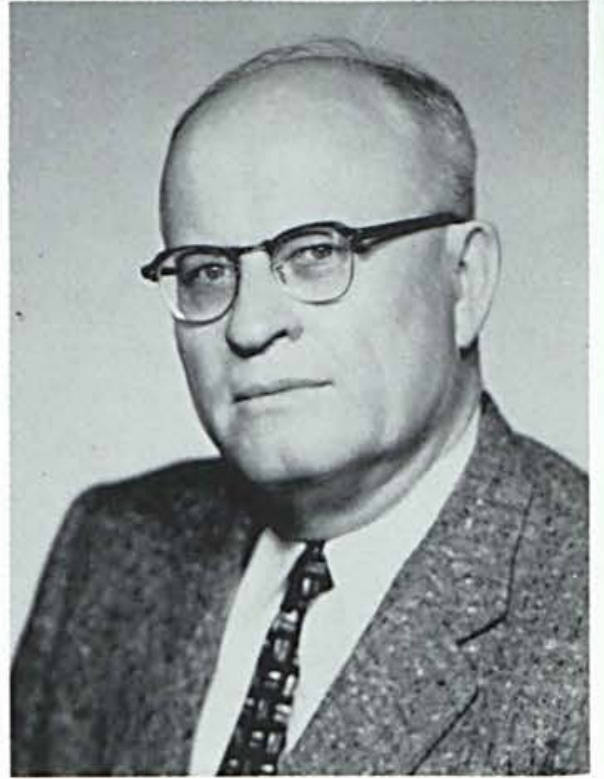

CLIFFORD R. MADDOX, Ph.D. Academic Dean

Psychology and Education

\section{Executive Committee}

George S. Milner, Chairman C. R. Maddox
George L. Boyd

Wm. Patterson

Earl Willetts
Wm. Brook

Wilbur Rooks Arthur Williams
James T. Jeremiah

Robert Underwood

\section{Board of Trustees}

Charles Barth

Joseph H. Bower

Glenn Davis

Herman W. Harvey

George S, Milner, Chairman

William B. Patterson

Gerald Smelser

Earl Willetts

Alfred Colwell
George A. Bates

George L. Boyd

John Draxler

James T. Jeremiah

Kenneth A. Muck

Wilbur C. Rooke

Eugene B. Smith

Donald Woodby
William Brock

Norman B. Chappell

Arthur Dyke

Allen E. Lewis

Dale Murphy

R. O. Sanborn

Charles Wallace

Thomas Younger 


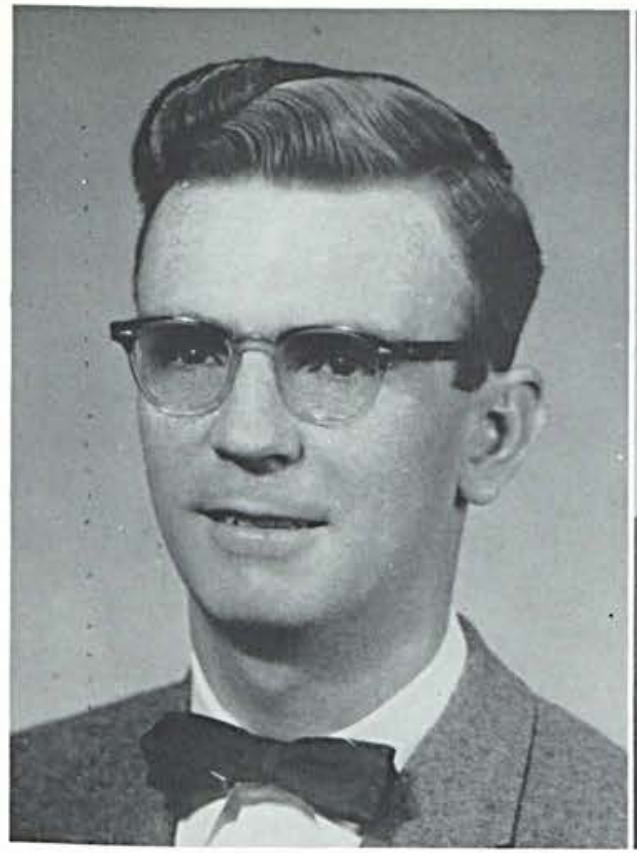

HARMON BERGEN, B.A.

English

Language

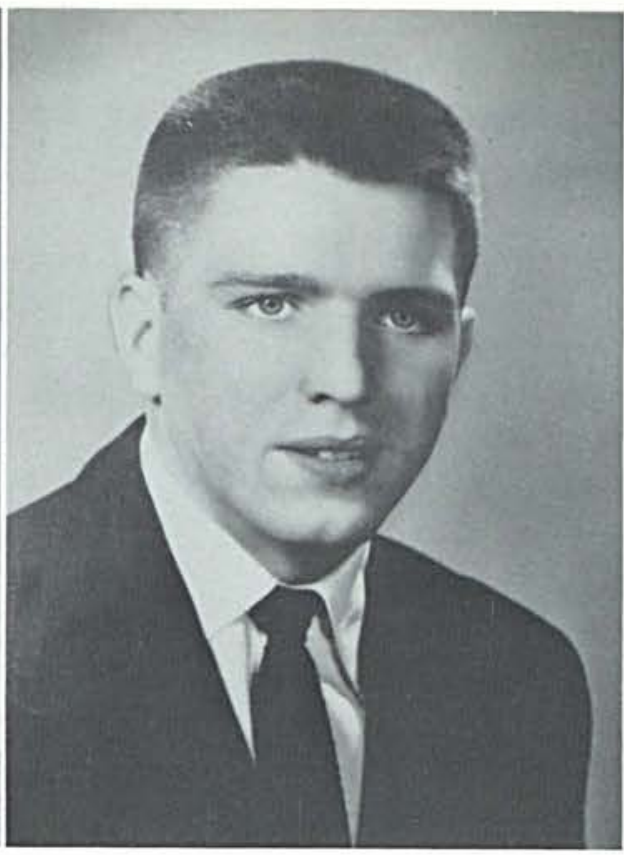

SHERWIN BOWSER, B.S.

Physical Education
RICHARD COOKE, M.S. Music
RUTH KANTZER, B.A. English
CLEVELAND McDONALD, M.Litt. Social Science

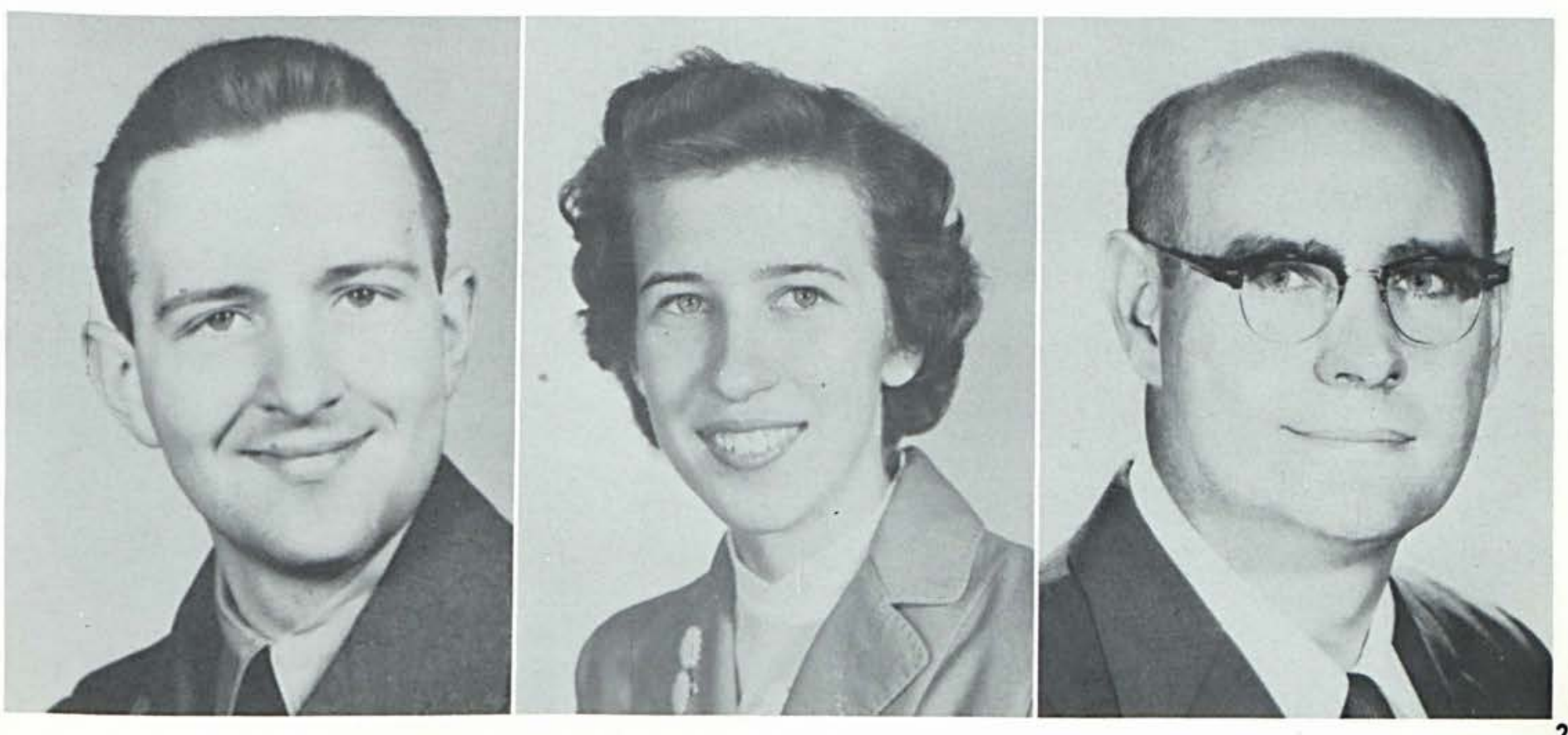




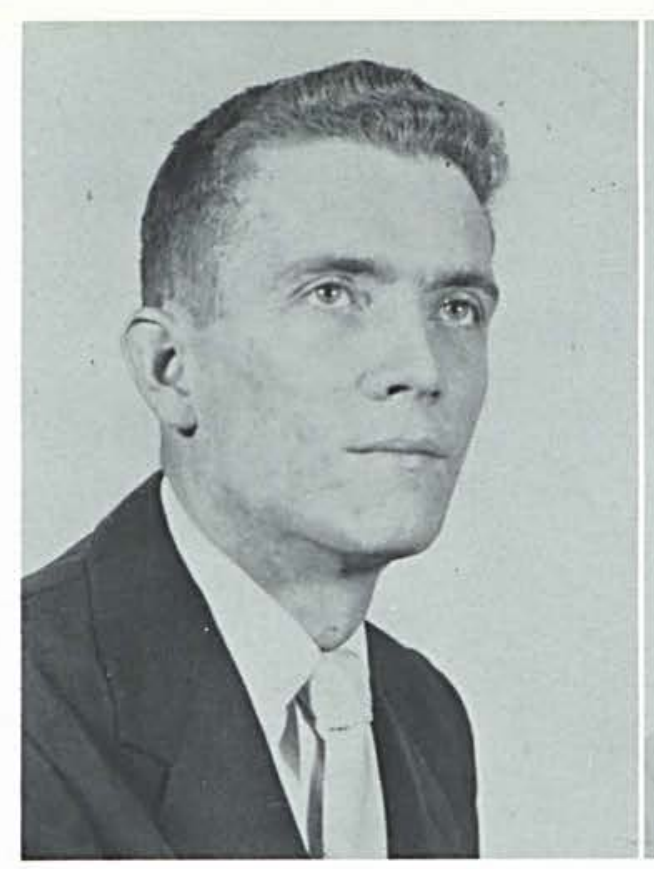

DALE THOMSON, B.A. Biological Science

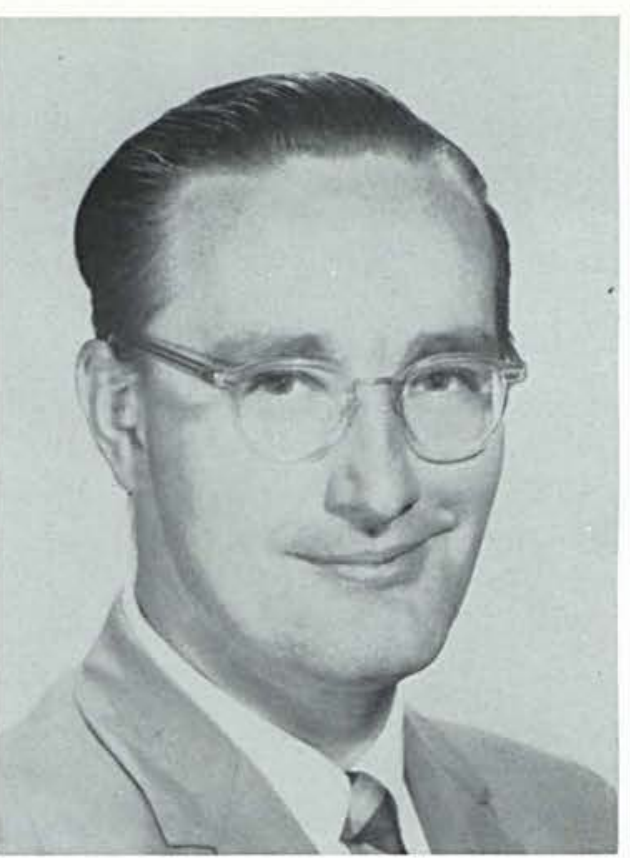

BRUCE TURNBULL, B.D., M.S. Chemical Science
WARREN WEBBER, M.Mus.Ed. Music
G. PAUL WYLAND, M.A. History
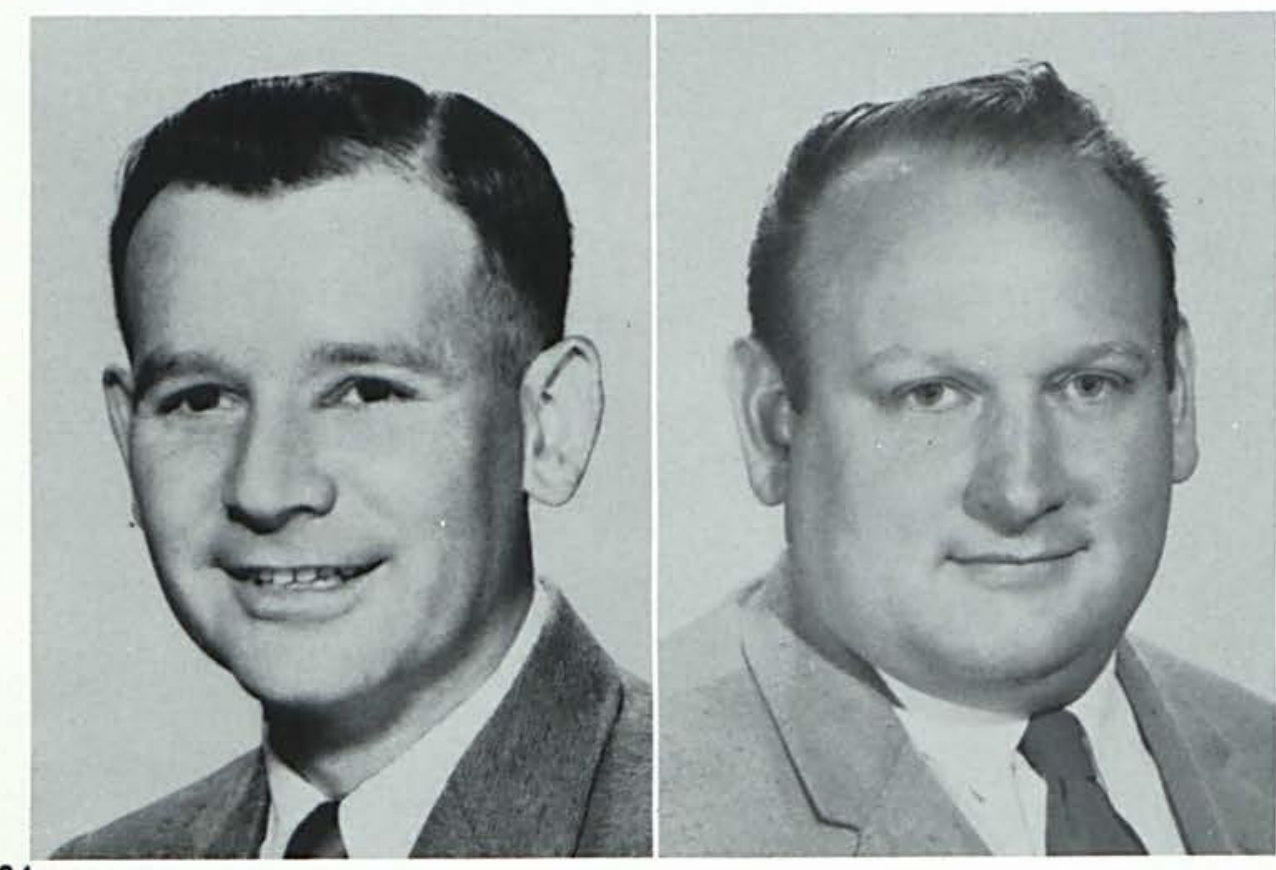


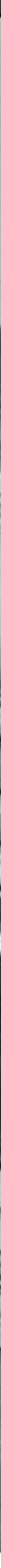




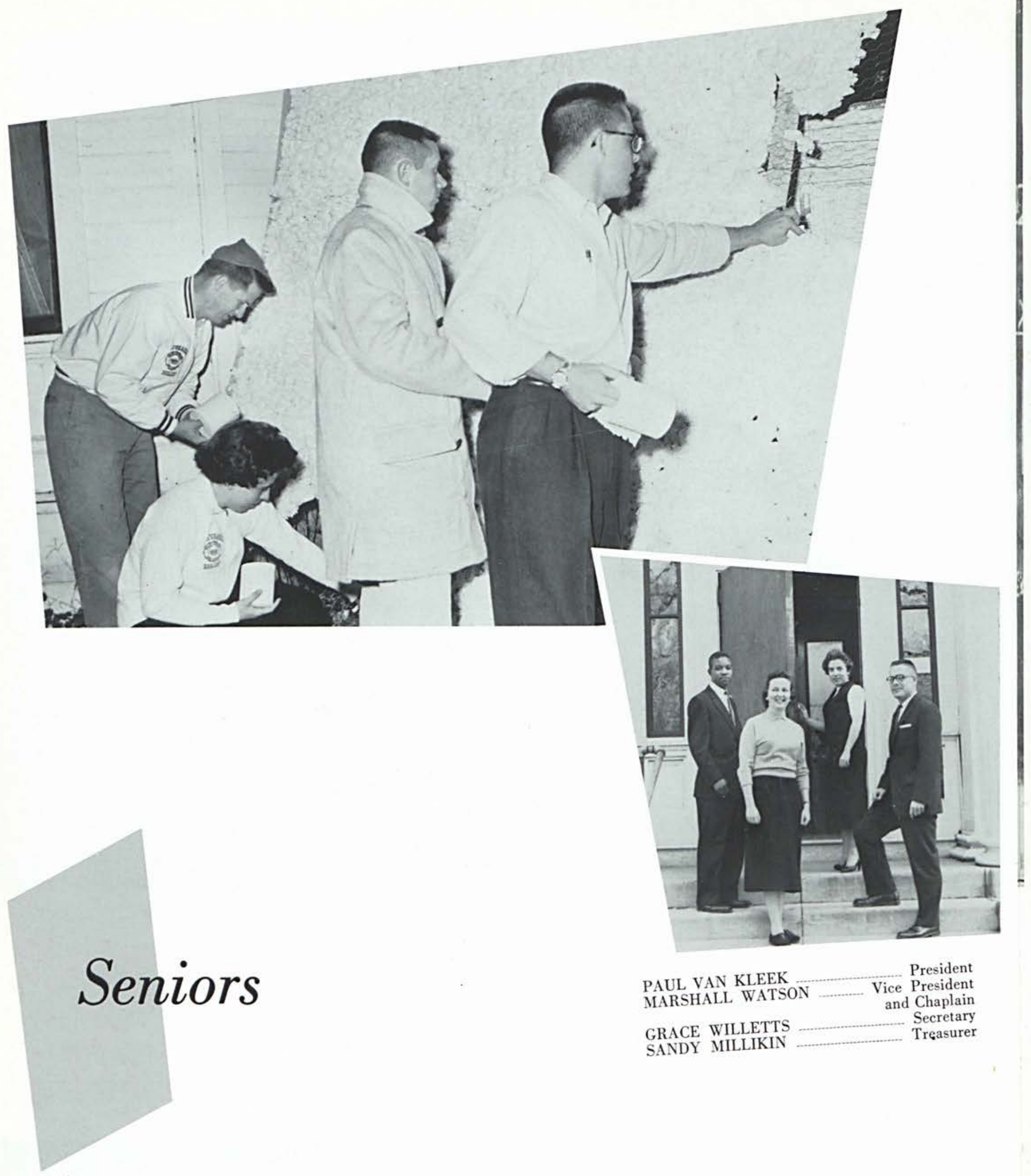

"The life that goes forward triumphs." 


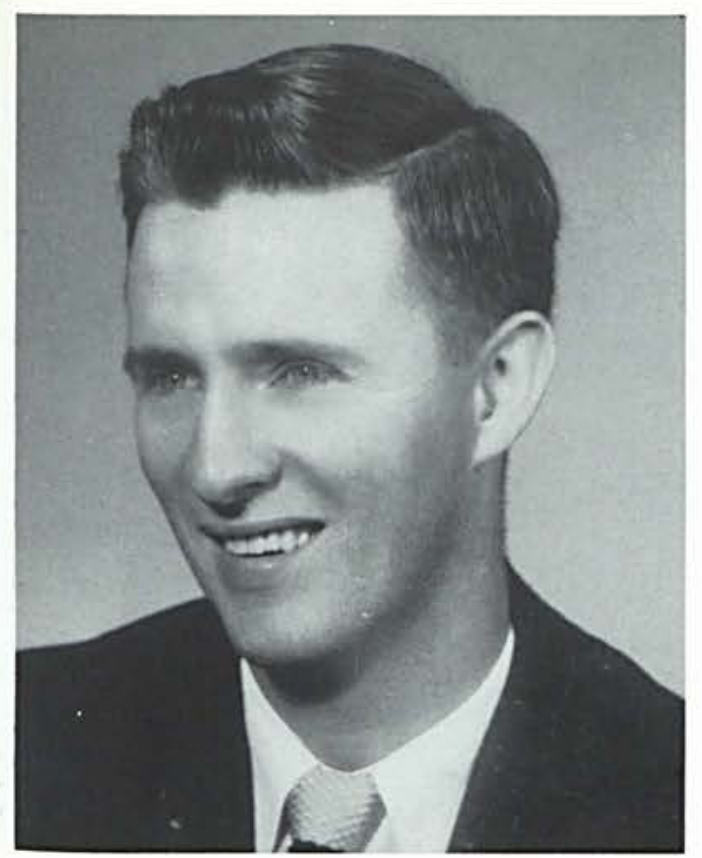

CLIFFORD BOESSEL

Hobart, Indiana

First Baptist Church

B.A., History

Class President 3; Basketball 1; Baseball 2.

Kalkaska, Michigan

Kalkaska Baptist Church

B.A., Social Science

\section{SANDRA MILLIKIN ENTNER}

Class Secretary 3, Treasurer 4; Miracle Staff 3; Whispering Cedars, Assistant Editor 2, 3; Trio 4; Choir 1, 2, 3, 4; Gamma Chi 2, Social Chairman 3, 4; Cheerleader 1, 2, 3, 4; Girls' Chorus 3; Pep Band 1, 2, 4; Girls' Basketball 1, 2, 3, 4 .
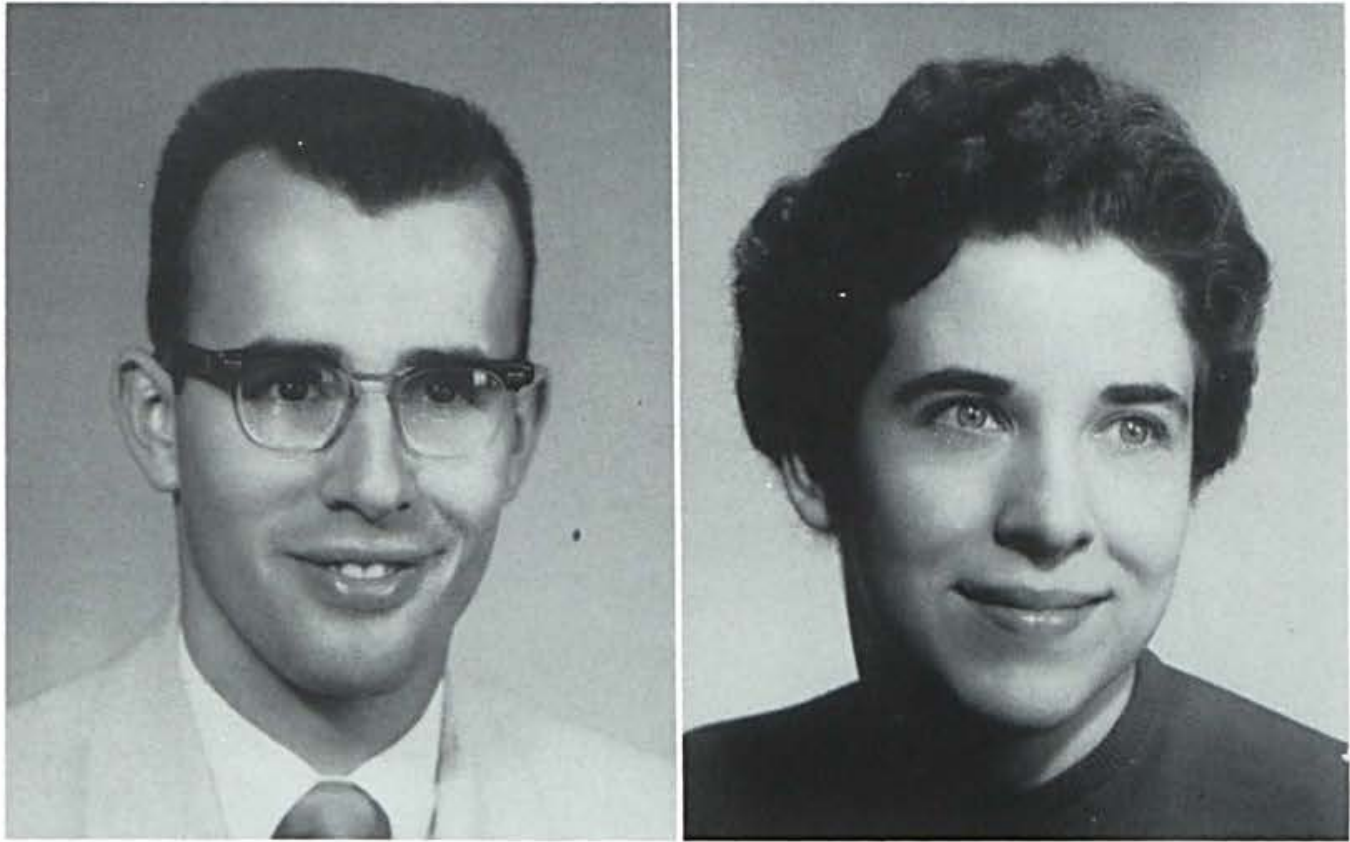

\section{EUGENE CHRISTIAN}

Cedarville, Ohio

Grace Baptist Church

B.A., History

Class President 1; Basketball 1; Miracle Staff 3;

Choir 1; Intramurals 2, 3, 4 .

\section{ROBERT HOWDER}

Angola, New York

First Baptist Church

B.S., Physical Science

Wheaton College Summer School

Class Vice President 3; Student Council 1, Treas. urer 4; Varsity "C" 3, Business Manager 4; Basketball 1, 2, 3, 4; Baseball Manager 2, 3; Miracle Staff 3; Trumpet Trio 3, 4; Alpha Chi 2, Secretary and Treasurer 3, President 4; Traffic Court 4.

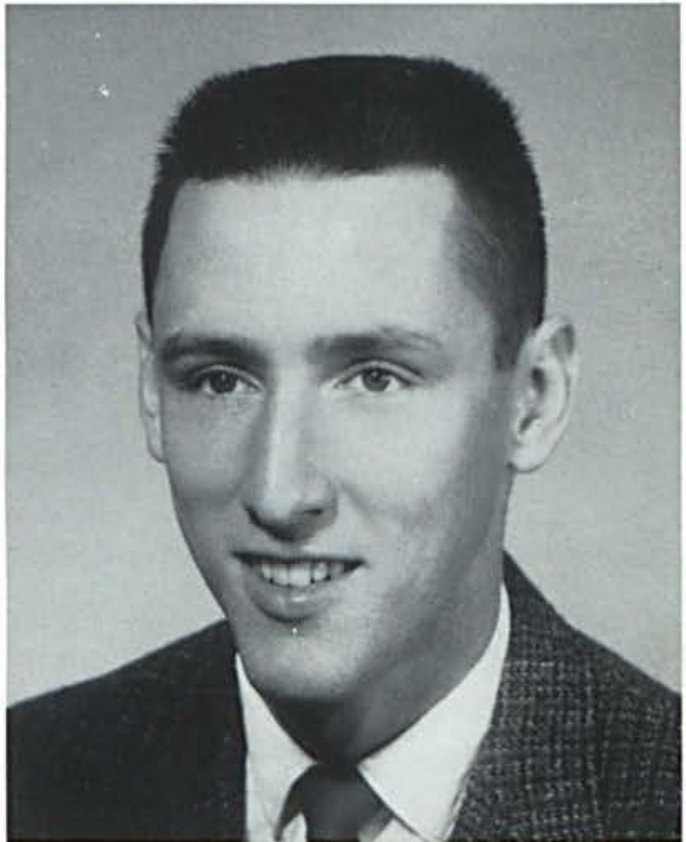


CAROL JOHNSON

West Palm Beach, Florida

Blessed Hope Baptist Church

B.A., Bible

Class Secretary 2, Treasurer 3; Student Council

Secretary 4; Miracle Staff 3; Girls' Chorus 3;

FWM Secretary 2, 4.

\section{RAYMOND REINER}

North Boston, New York Hamburg Baptist Church

B.A., Bible

Miracle Staff 3; FWM President 4.
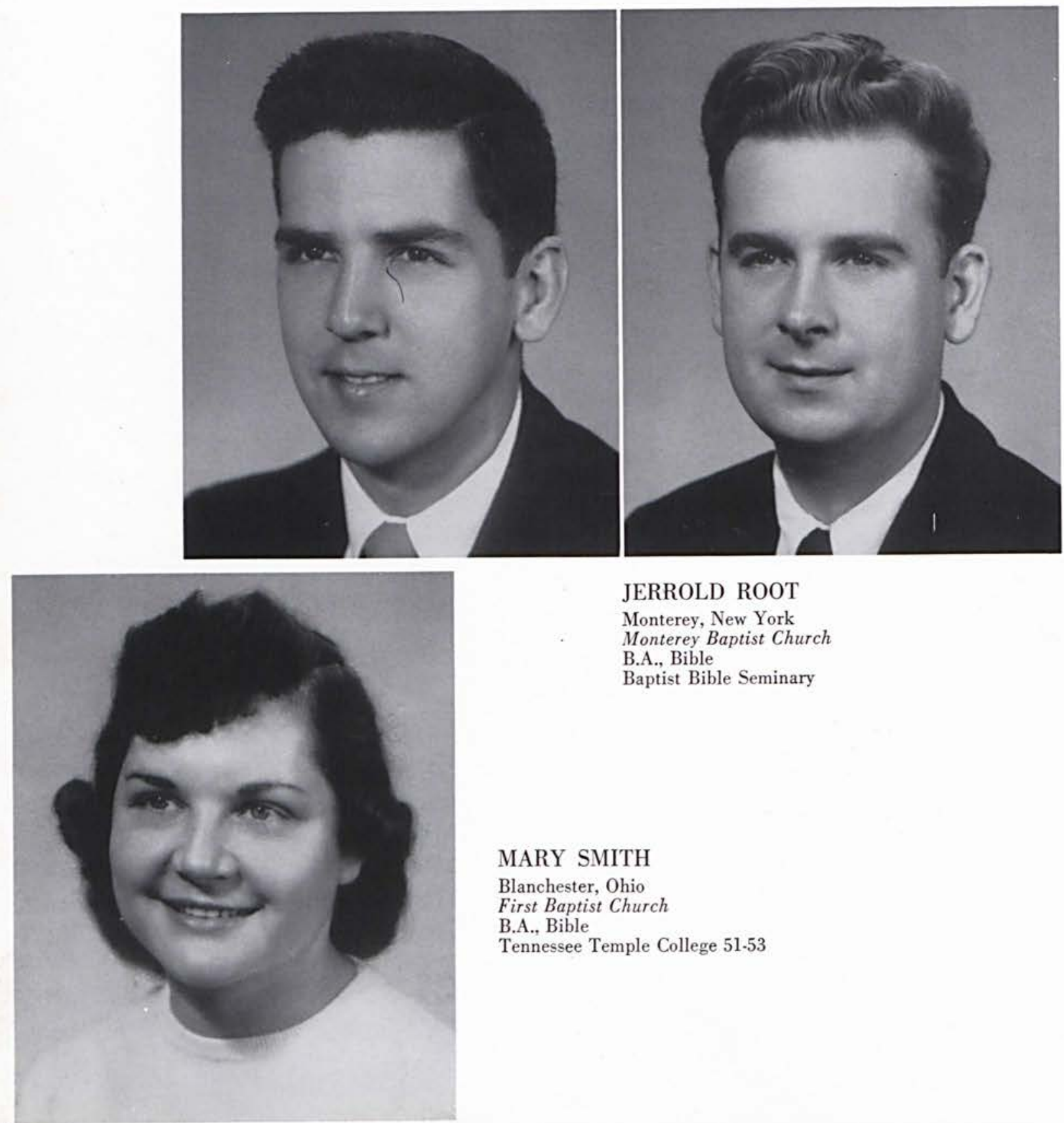

\section{MARY SMITH}

Blanchester, Ohio

First Baptist Church

B.A., Bible

Tennessee Temple College 51-53

JERROLD ROOT

Monterey, New York

Monterey Baptist Church

B.A., Bible

Baptist Bible Seminary

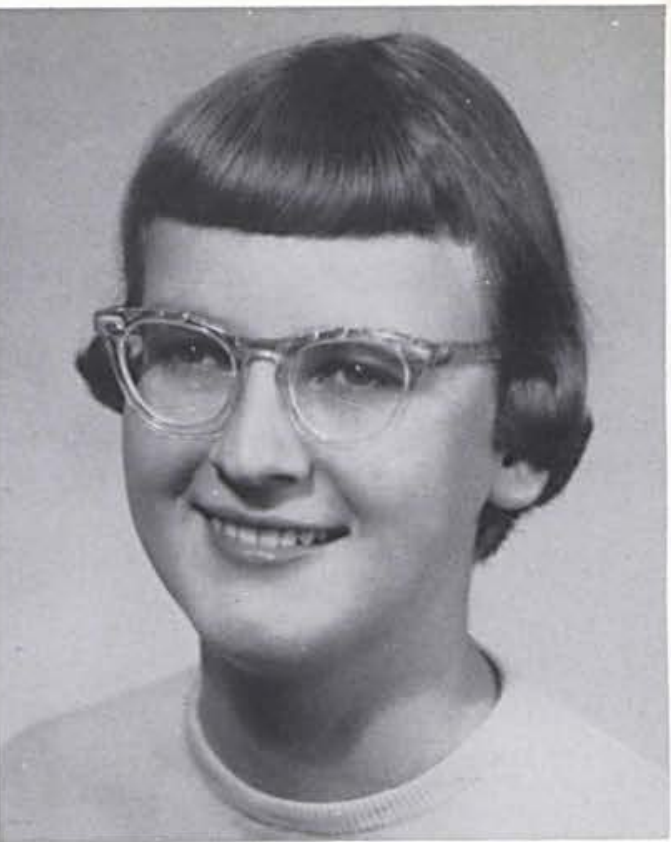




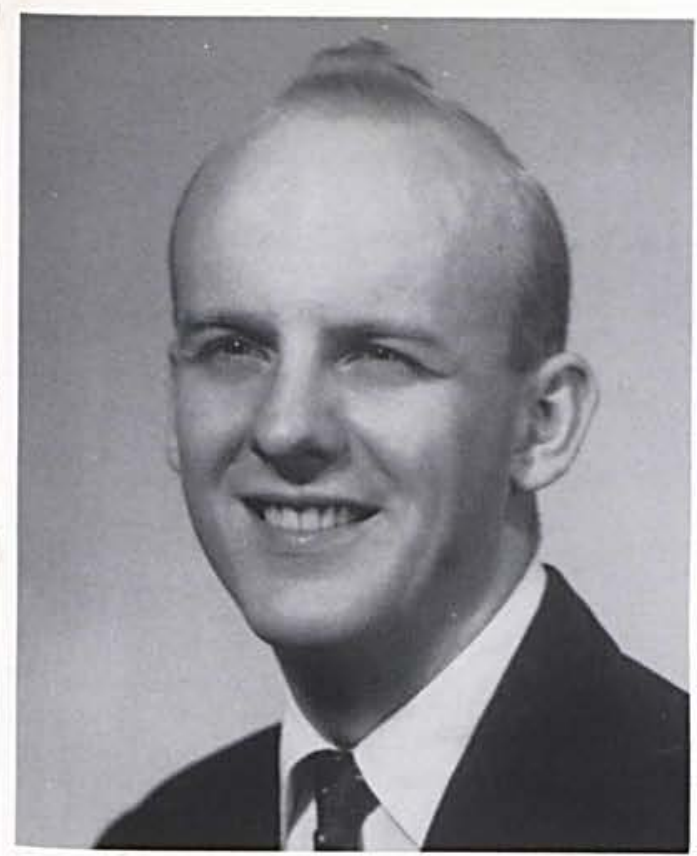

\section{DAVID THOMAS}

Dayton, Ohio

Christian Missionary Alliance

B.S., General Science

Student Council 3, 4; Varsity "C" 2, Secretary 3

and 4; Basketball Manager 1, 2, 3, 4; Baseball

Manager 1, 2, 3, 4; Whispering Cedars 4; Alpha

Chi 2, 3, Vice President 4; Student Court 3, 4;

Chairman of Election Board 4; FWM 4; Home-

coming Play 4.

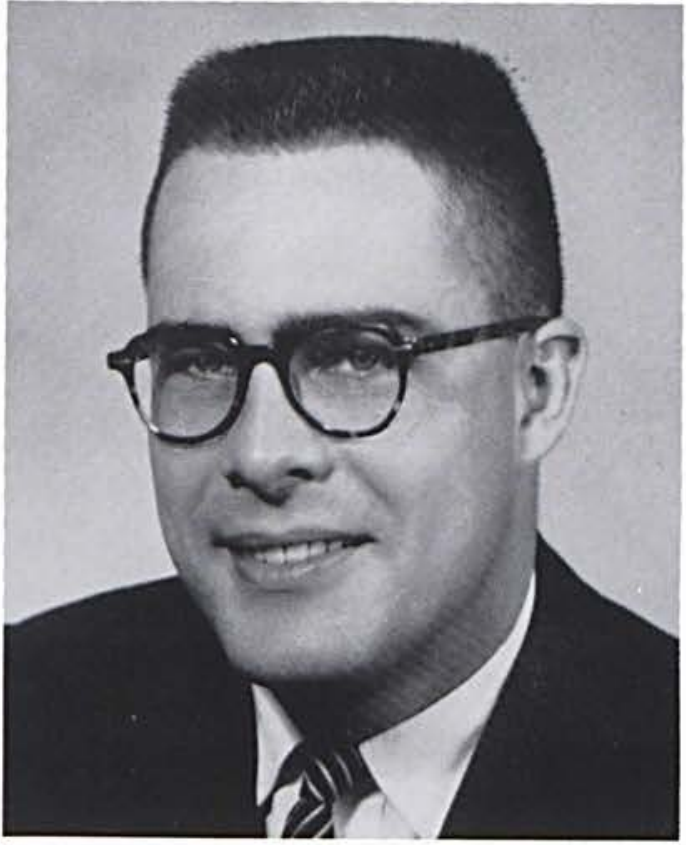

\section{PAUL VAN KLEEK}

Lapeer, Michigan

First Baptist Church

B.A., Social Science

Class President 4; Student Council 2,

President 3; Discipline Committee 3;

Miracle Staff 3; Whispering Cedars 4; Choir 1, 3, 4; Alpha Chi 2, 3, 4; Pep Band 1, 2, 3, 4; Tract Club President 1.

MARSHALL WATSON JR.

Cedarville, Ohio

Zion Baptist Church

B.A., History

Class President 1, Chaplain 2, Vice President 4, Chaplain 4.

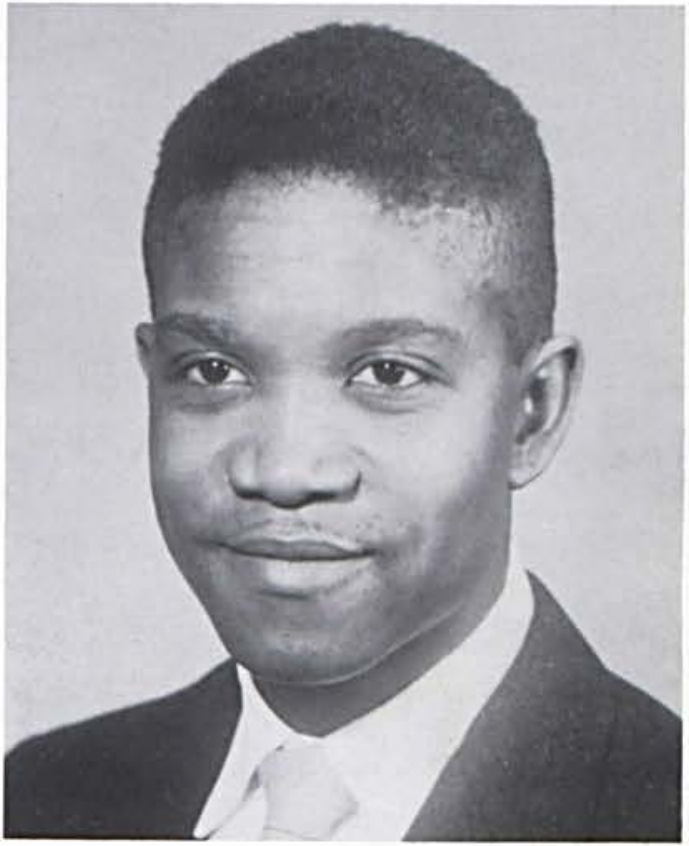




\section{LES WEBSTER}

Hamburg, New York First Baptist Church

B.A., Bible

Class Vice President 2, Chaplain 3; Varsity "C" 4; Basketball Statistician 3; Tennis 2; Alpha Chi, Program Chairman 1, 2, 3, 4; FWM Vice President 1.

GRACE WILLETTS

\section{Berea, Ohio}

Berea Baptist Church

B.A., Music

Class Treasurer 2, Secretary 4; Student Council 3, 4; Discipline Committee 3, 4; Miracle Staff 1, 3; Trio 1, 2, 3, 4; Choir 1, 2, 3, 4; Music Masters 3; Student Council 3, 4; Gamma Chi 2, 3, Vice President 4; Girls' Chorus 3; Social Committee 1, 2; Homecoming Play 3, 4; Women's Council Vice President 3, 4; Girls' Basketball 1 .
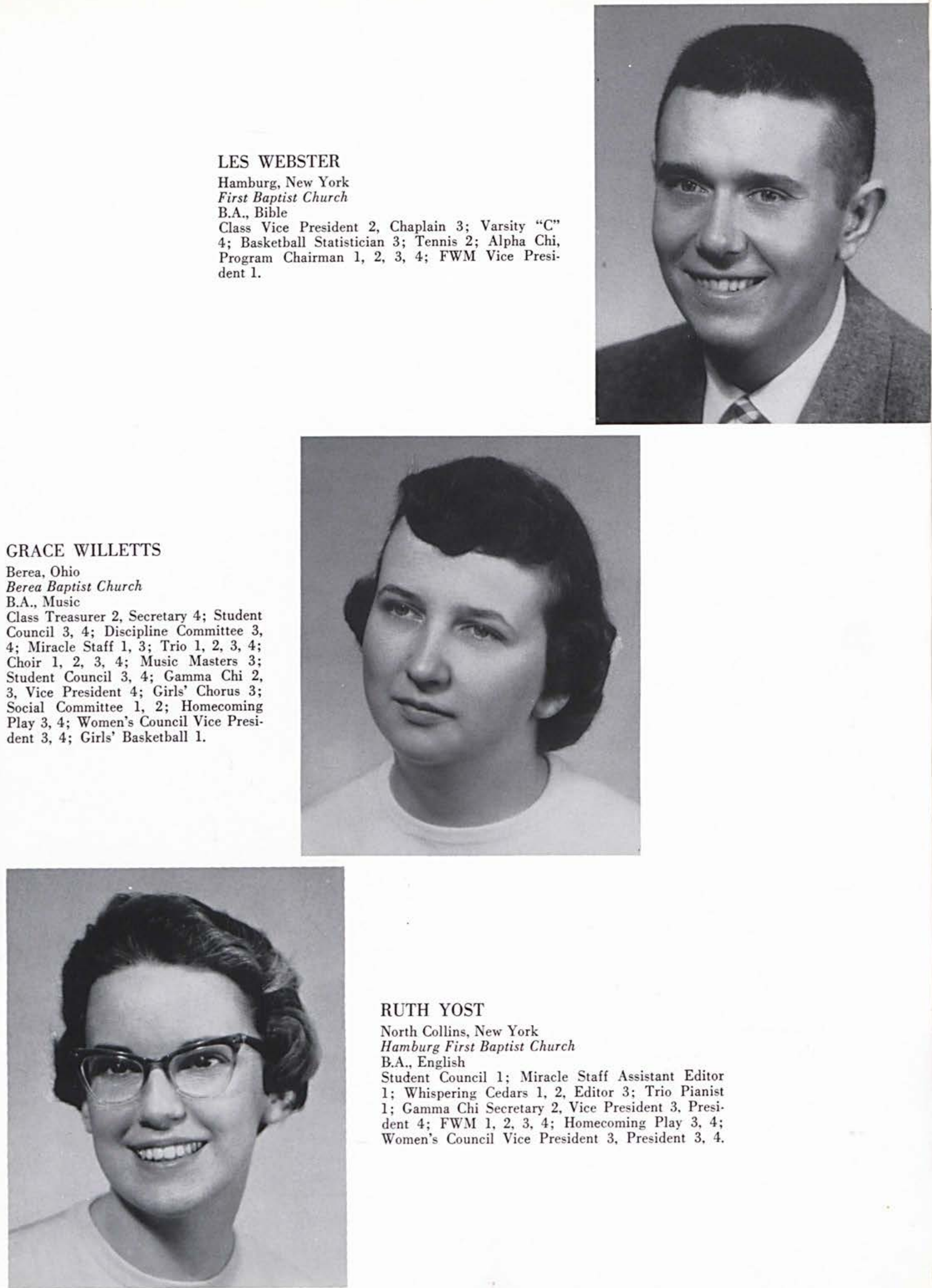

\section{RUTH YOST}

North Collins, New York

Hamburg First Baptist Church

B.A., English

Student Council 1; Miracle Staff Assistant Editor

1; Whispering Cedars 1, 2, Editor 3; Trio Pianist

1; Gamma Chi Secretary 2, Vice President 3, President 4; FWM 1, 2, 3, 4; Homecoming Play 3, 4;

Women's Council Vice President 3, President 3, 4. 


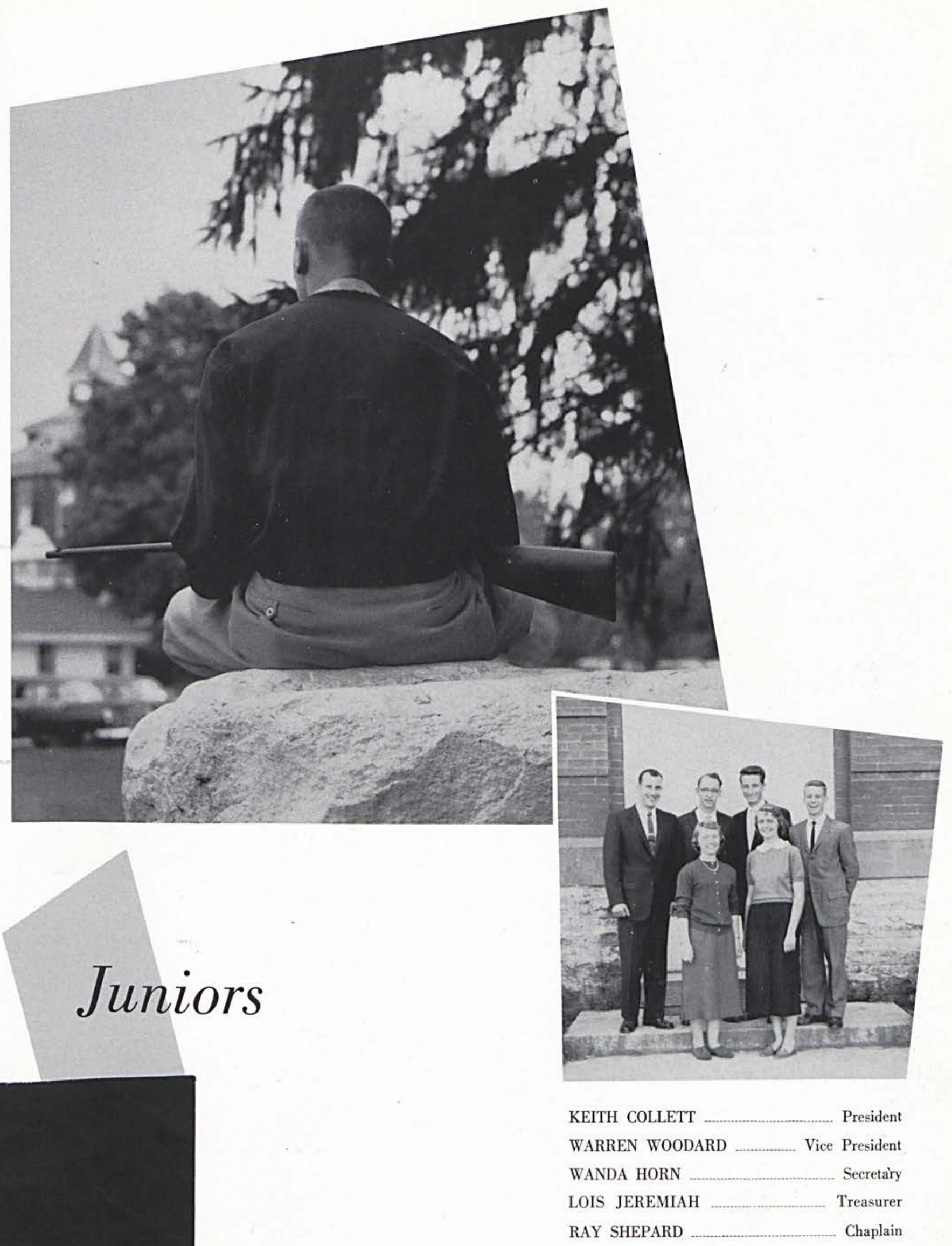

"To God the Glory, to men the Gospel." 


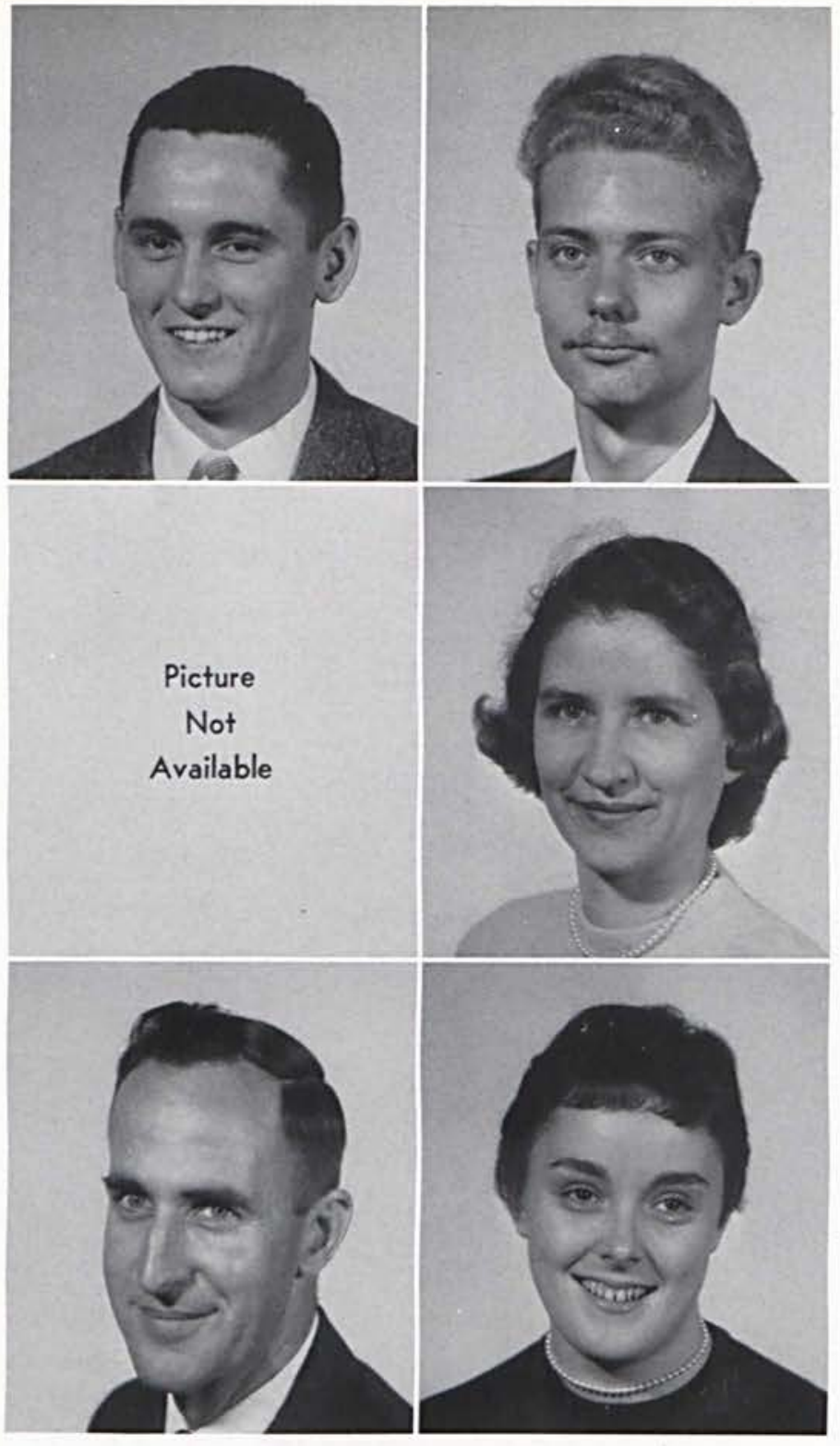

KEITH COLLETT

Cedarville, Ohio

DAVID DAUTEL

Portsmouth, Ohio

Temple Baptist Church

KEN DOCTOR

Ellsworth, Michigan

Eastport Baptist Church

JOHN ENTNER

Connersville, Indiana

Community Baptist Church
MERLIN AGER

Verona. Wisconsin

Memorial Baptist Church

PAUL ANDERSON

Gary, Indiana

Miller Baptist Church

ROY CARR

Sinclairville, New York

First Baptist Church

ALBERTA CHAFFE

Thompson. Ohio

South Madison Community Church

STUART CHAFFE

Thompson, Ohio

South Madison Community Church

\section{KAREN COLLETT}

Cedarville, Ohio

Berea Baptist Church

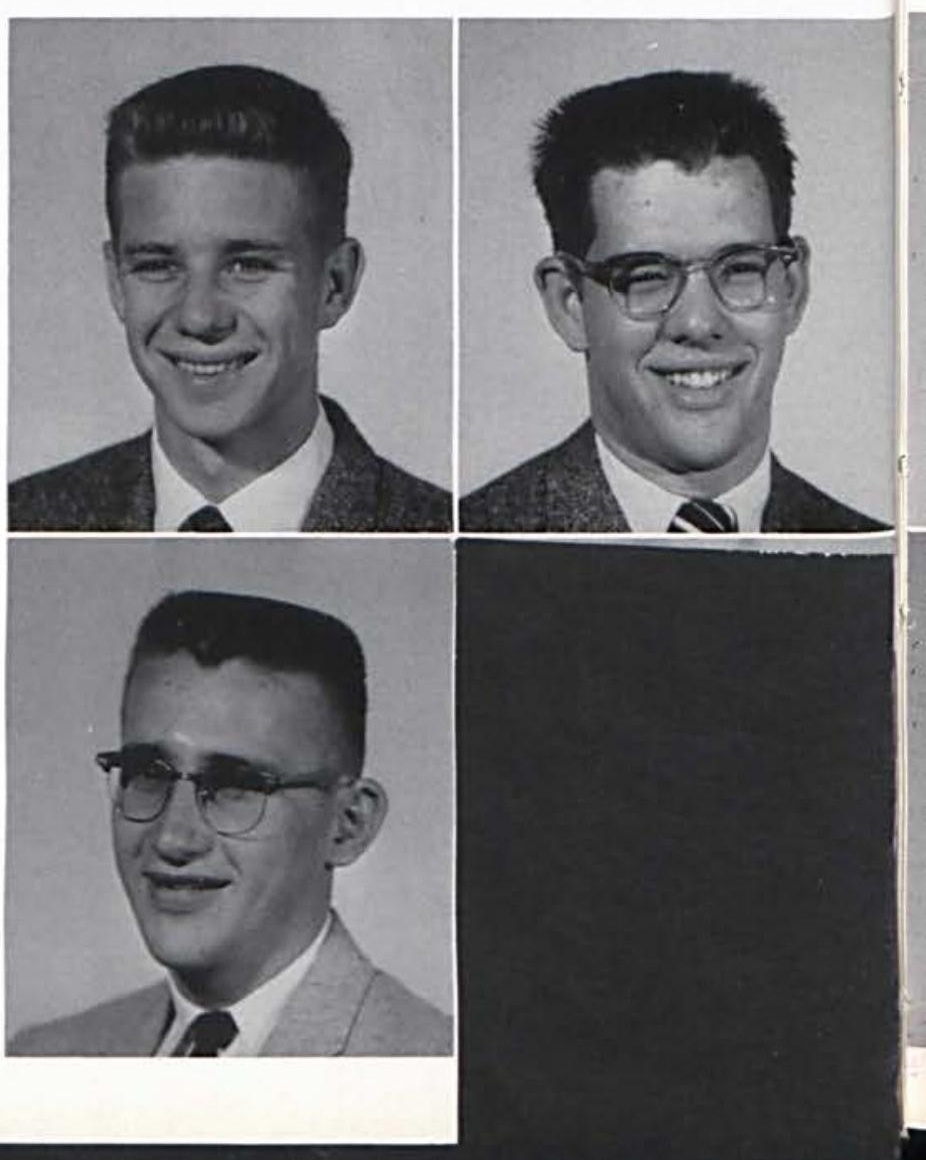




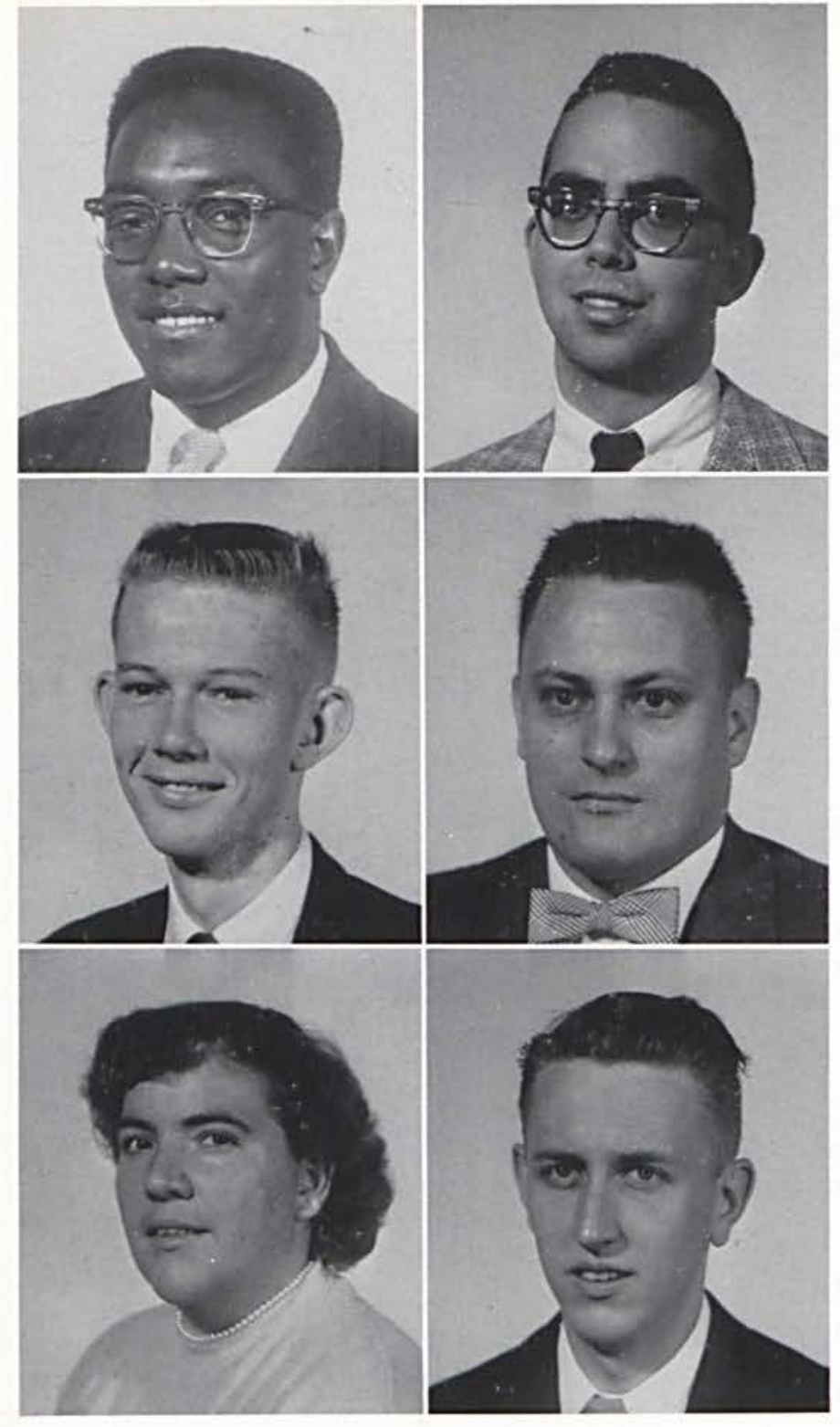

WARREN PROUD

Grand Rapids, Michigan

Trinity Baptist Church

PETE REESE

Cedarville, Ohio

Clifton Presbyterian Church
WILLIAM MASON

Columbus, Ohio

Vallejo Bible Church

DAVE MATSON

Plymouth, Indiana

First Baptist Church

DAVID MULDER

Donna, Texas

Calvary Baptist Church

JAMES NEELY

Glenview, Illinois

Community Baptist Church
NORMA NULPH

Climax, Pennsylvania

Climax Baptist Church

KENNETH PIERPONT

Grandview, Illinois

Emmanuel Baptist Church
SALLY ROUDYBUSH Butler, Pennsylvania

First Baptist Church

LEON ROWLAND

Springfield, Ohio

Blessed Hope Baptist Church

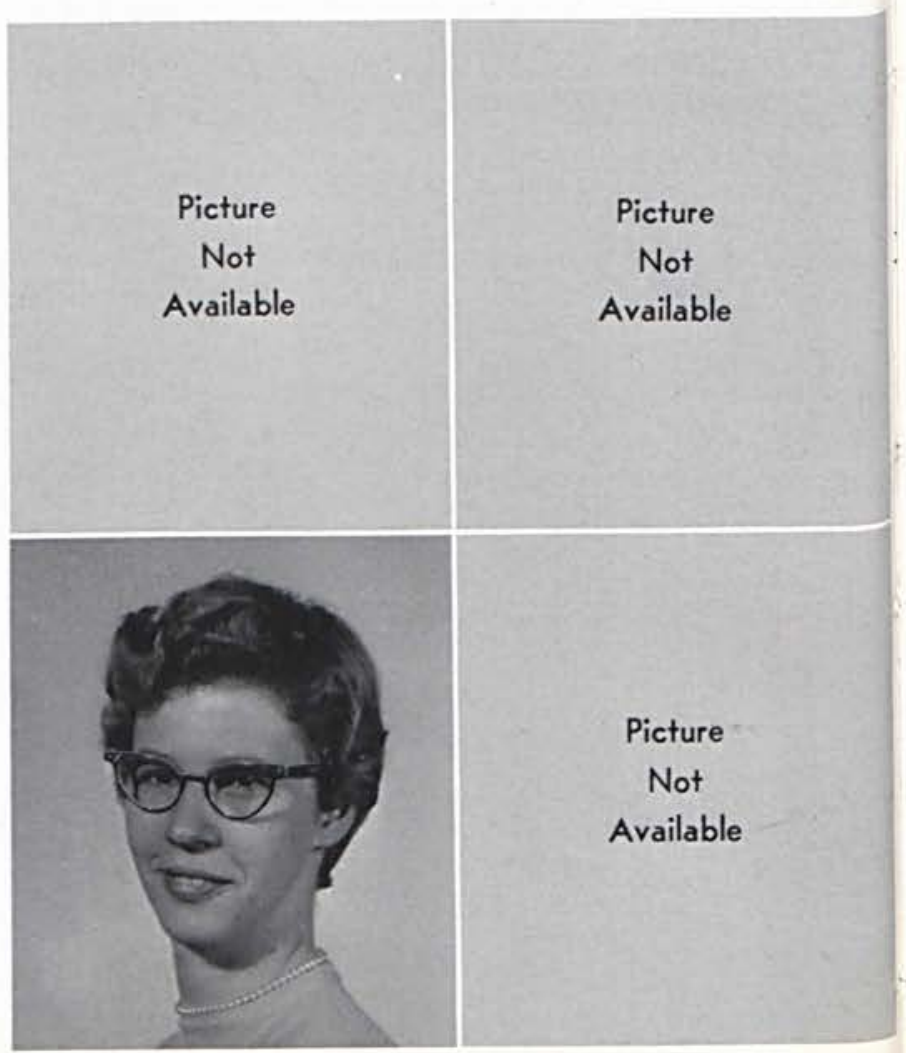


DONALD SEWELL

Batavia, Ohio

RAY SHEPHERD

Cincinnati, Ohio

Norwood Baptist Church

BARBARA SHERRY

Worthington, Pennsylvania

First Baptist Church

RUTH SMELSER

Cleveland Heights, Ohio

Bethlehem Baptist Church

LARRY SMITH

Macon, Illinois

Riverside Baptist Church

ROSEMARY SMITH

Kirkersville, Ohio

Reynoldsburg Bible Mission

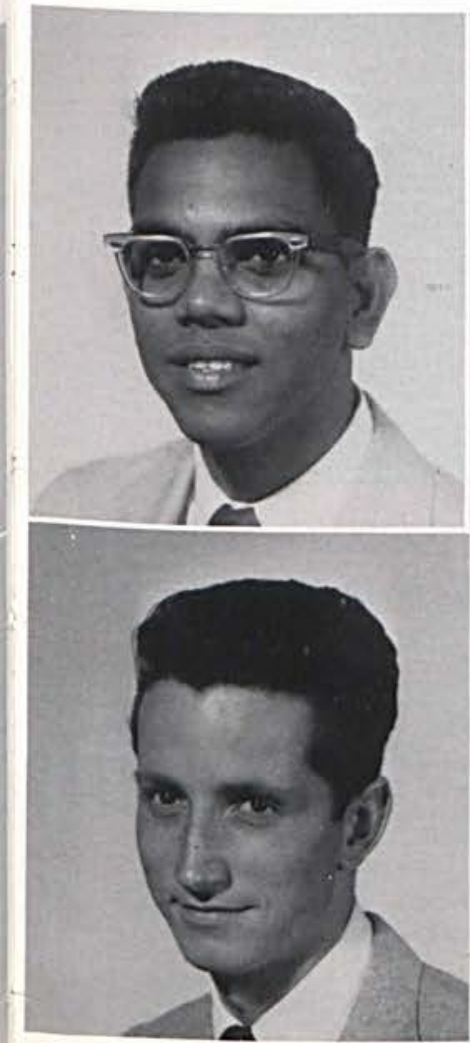

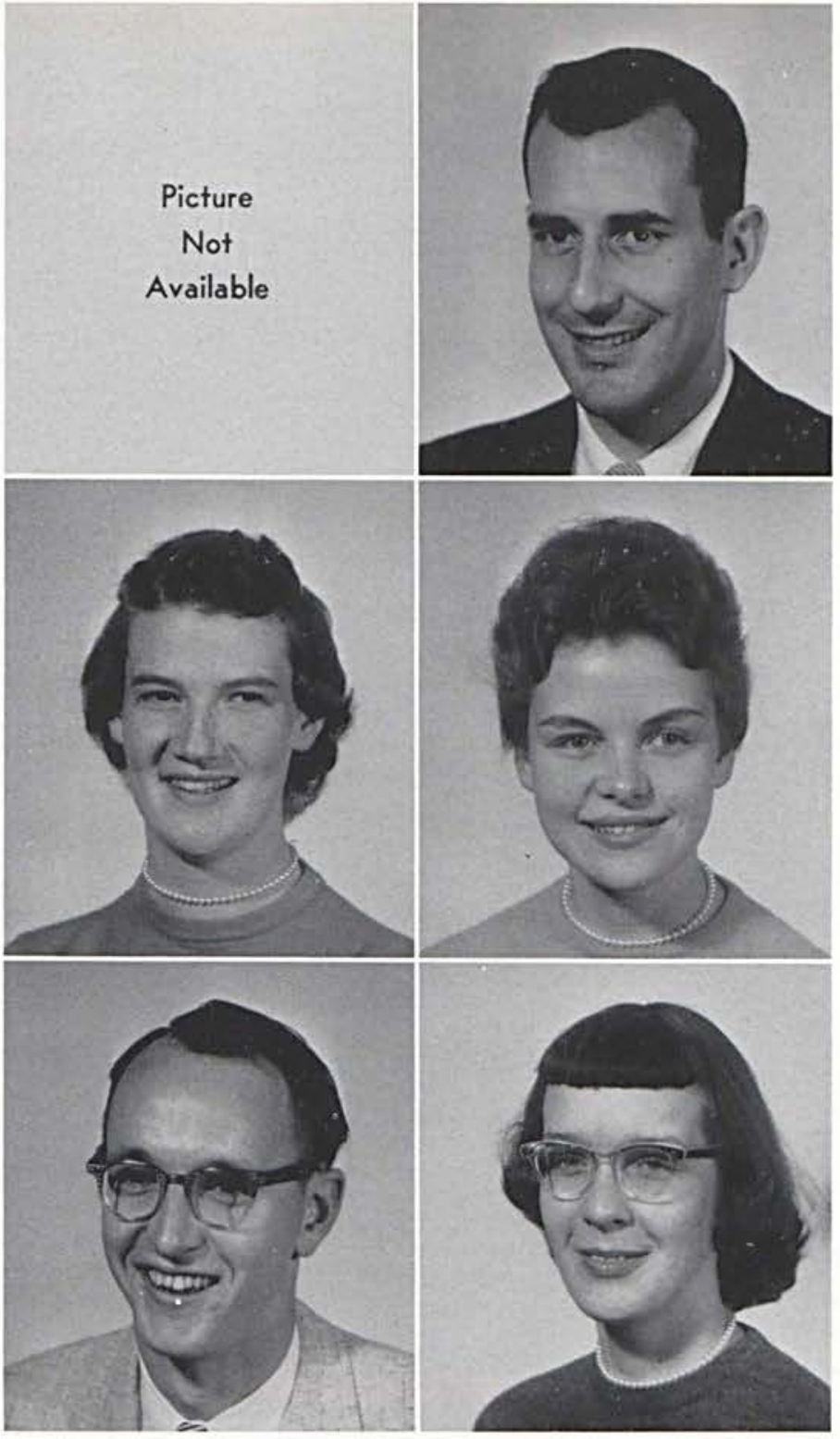

HUBLALL SOOKRAM

Corentyne, British Guiana

First Regular Baptist

HORACE WARD

Springfield, Ohio

Pentecostal Church
WARREN WOODARD

Jamestown, New York

Chandler St. Baptist Church 


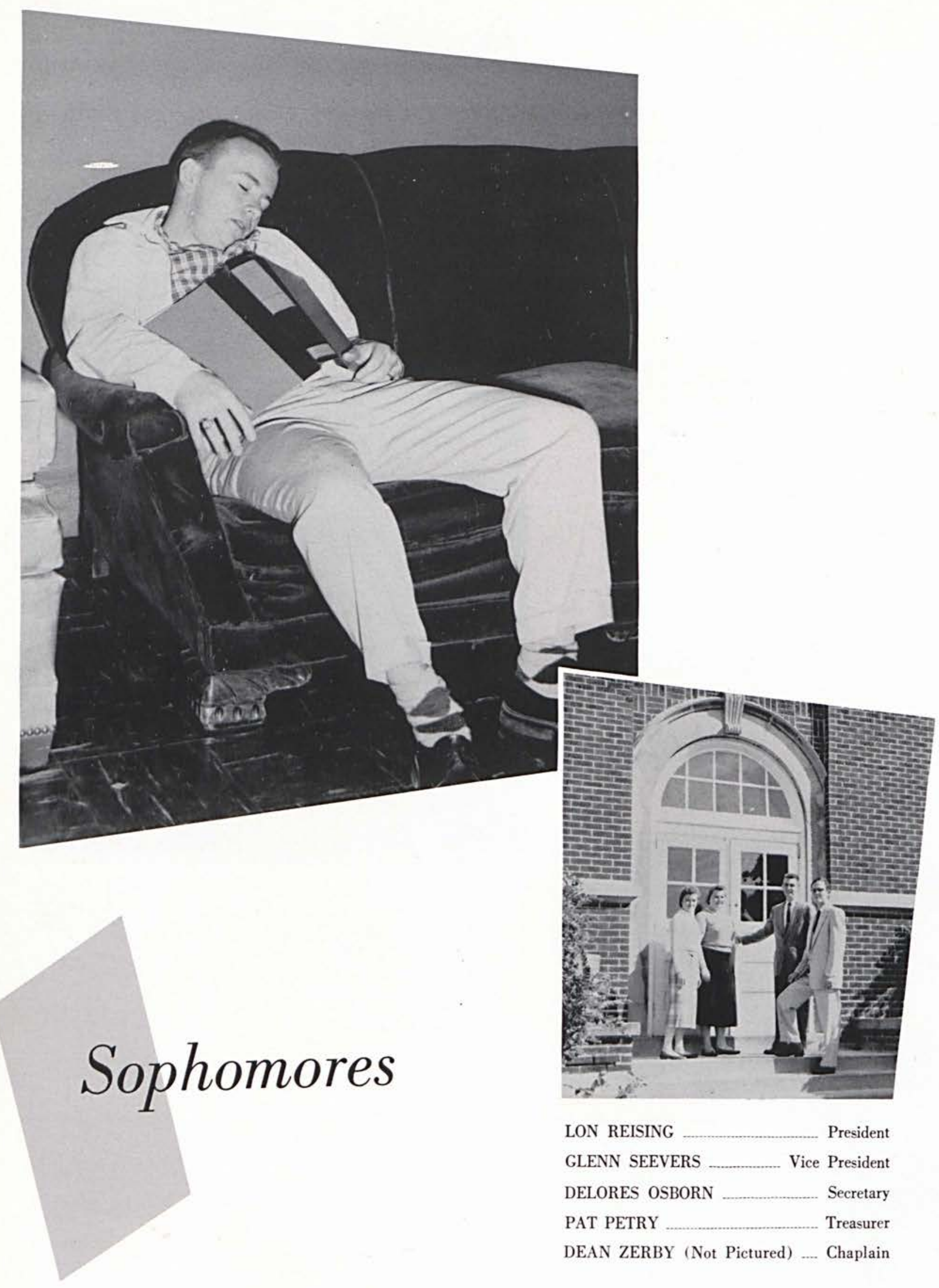

"With eternity's values in view" 


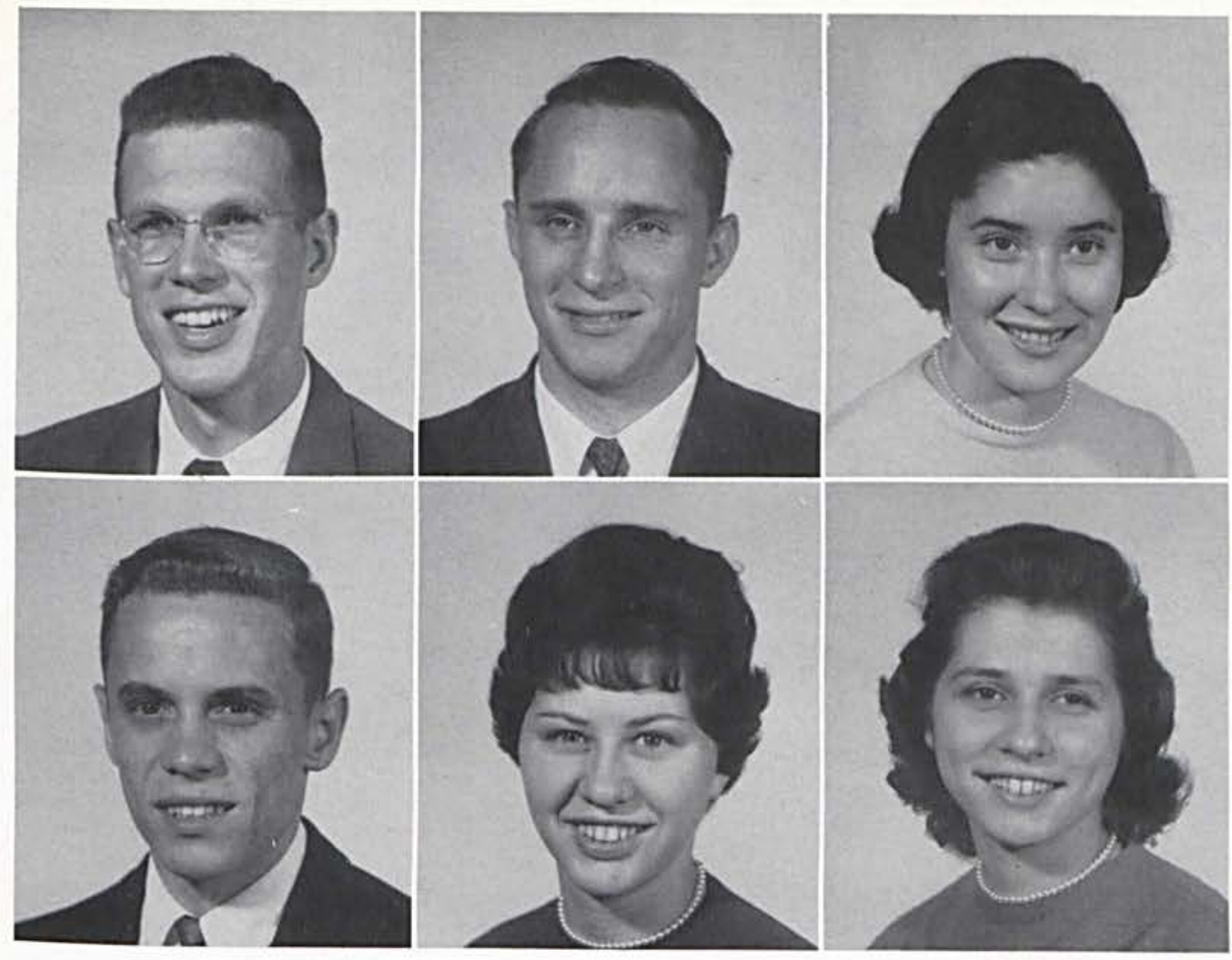

JOHN BUTLER

Greene, Iowa

Alameda Baptist Church

DAVID COCHRAN

Russell, Iowa

Center Community

DONIS COLLIER

Moweaqua, Illinois

Grace Baptist Church

DICK COOK

Flint, Michigan

Riverdale Baptist Church

MARCIA CROTHERS

Lakeville, Indiana

First Baptist Church

MARLENE DAVIS

Parma, Ohio

First Baptist Church

PHYLLIS DOBBS

Kuna, Idaho

First Baptist Church

LOIS DODSON

Winona Lake, Indiana

Winona Lake Baptist Church

JACK DOWDEN

Ames, Iowa

Campus Baptist Church

JAMES ENTNER

Connersville, Indiana

Community Baptist Church

CHARLES FRY

Crawfordsville, Indiana

First Baptist Church

TERRY GOODRICH

Boise, Idaho

Baptist Temple

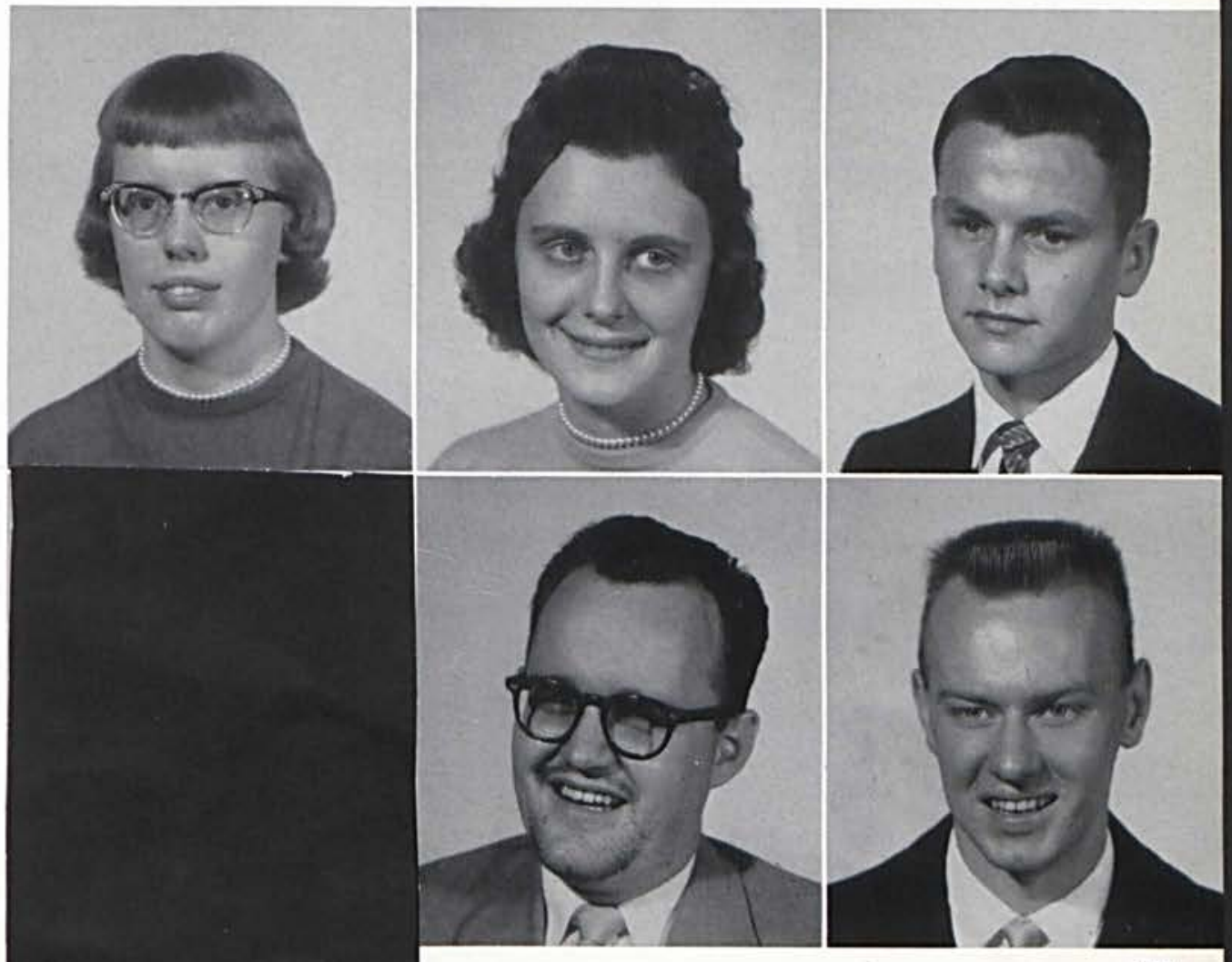




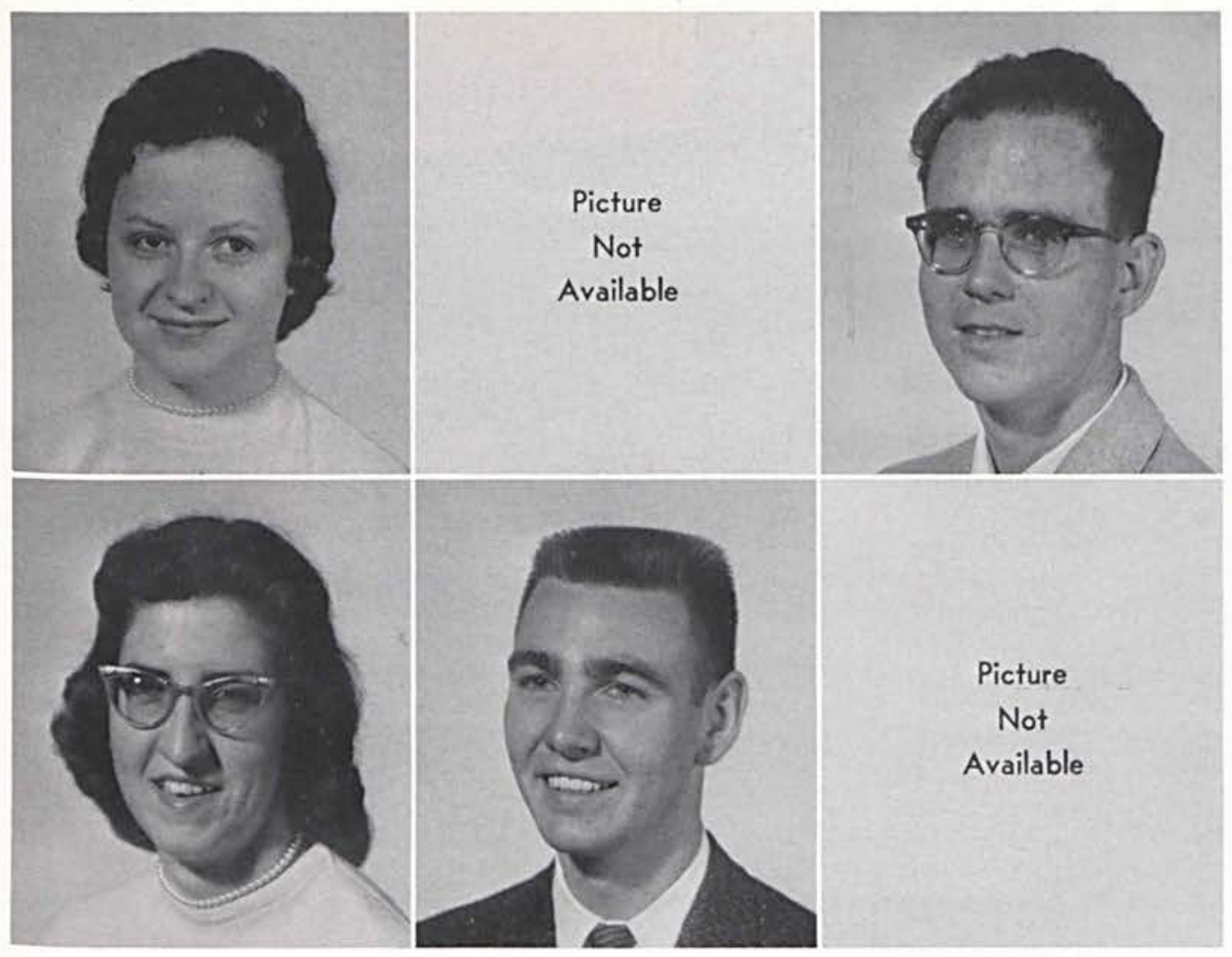

PAT PETRY

New Madison, Ohio

Immanuel Baptist Church

DAN PURDY

Columbia Station, Ohio

Berea Baptist Church

LON REISING

Chesterfield, Missouri

Lackland Road Baptist

SHARON ROUSH

Flint, Michigan

Riverdale Baptist Church

GLENN SEEVERS

Cleveland, Ohio

Parma Heights Baptist

ROBERT SELF

Toledo, Ohio

Emmanuel Baptist Church

ESTHER WEISS

Fairview Park, Ohio

Berea Baptist Church

WILLARD WENTZEL

Lorain, Ohio

DEAN ZERBY

Berrien Springs, Michigan

TERRY ZERBY

Berrien Springs, Michigan

CAROL ZOELLNER

Sandusky, Ohio

Woodlawn Baptist Church

RONALD ZOELLNER

Sandusky, Ohio

Woodlawn Baptist Church
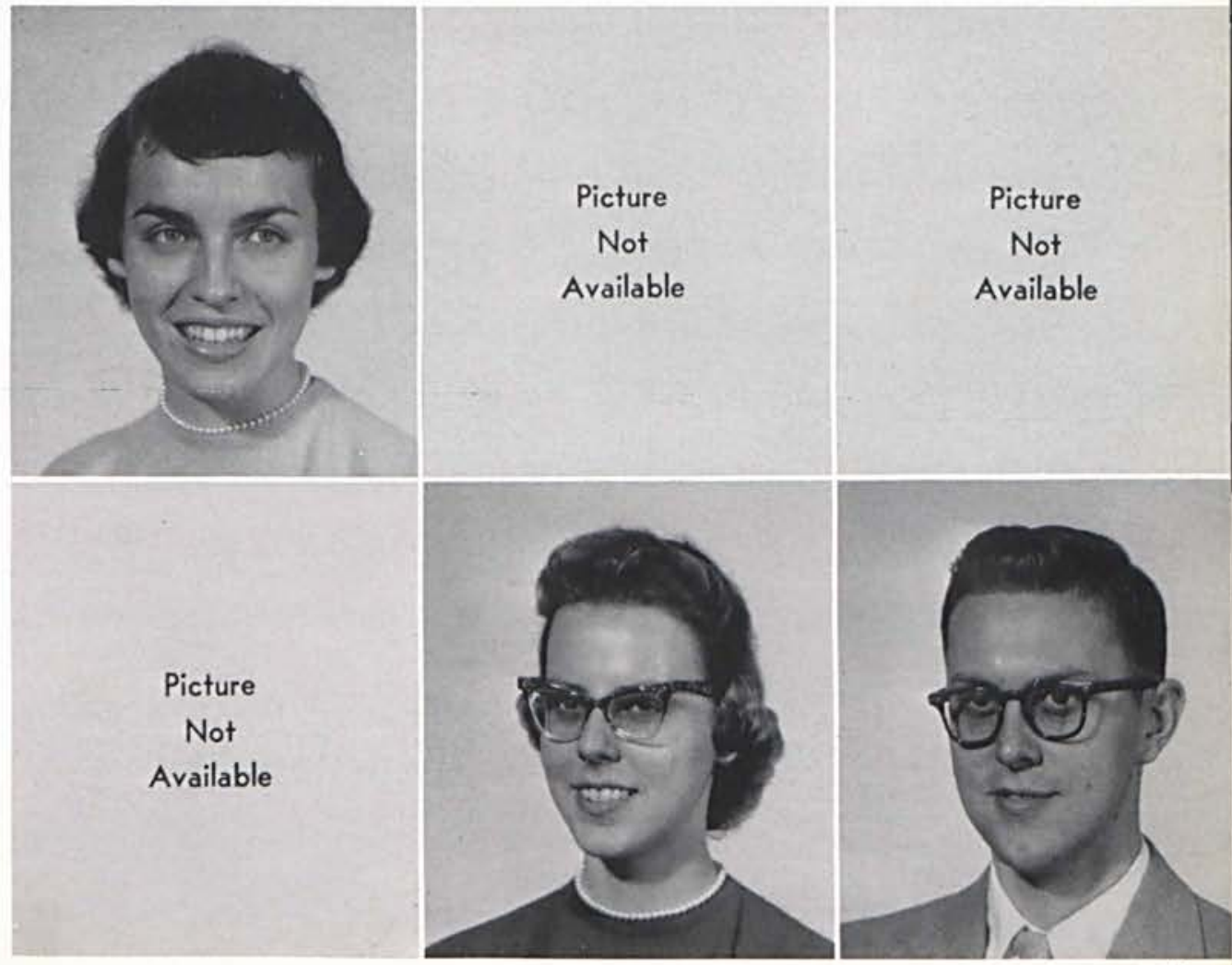


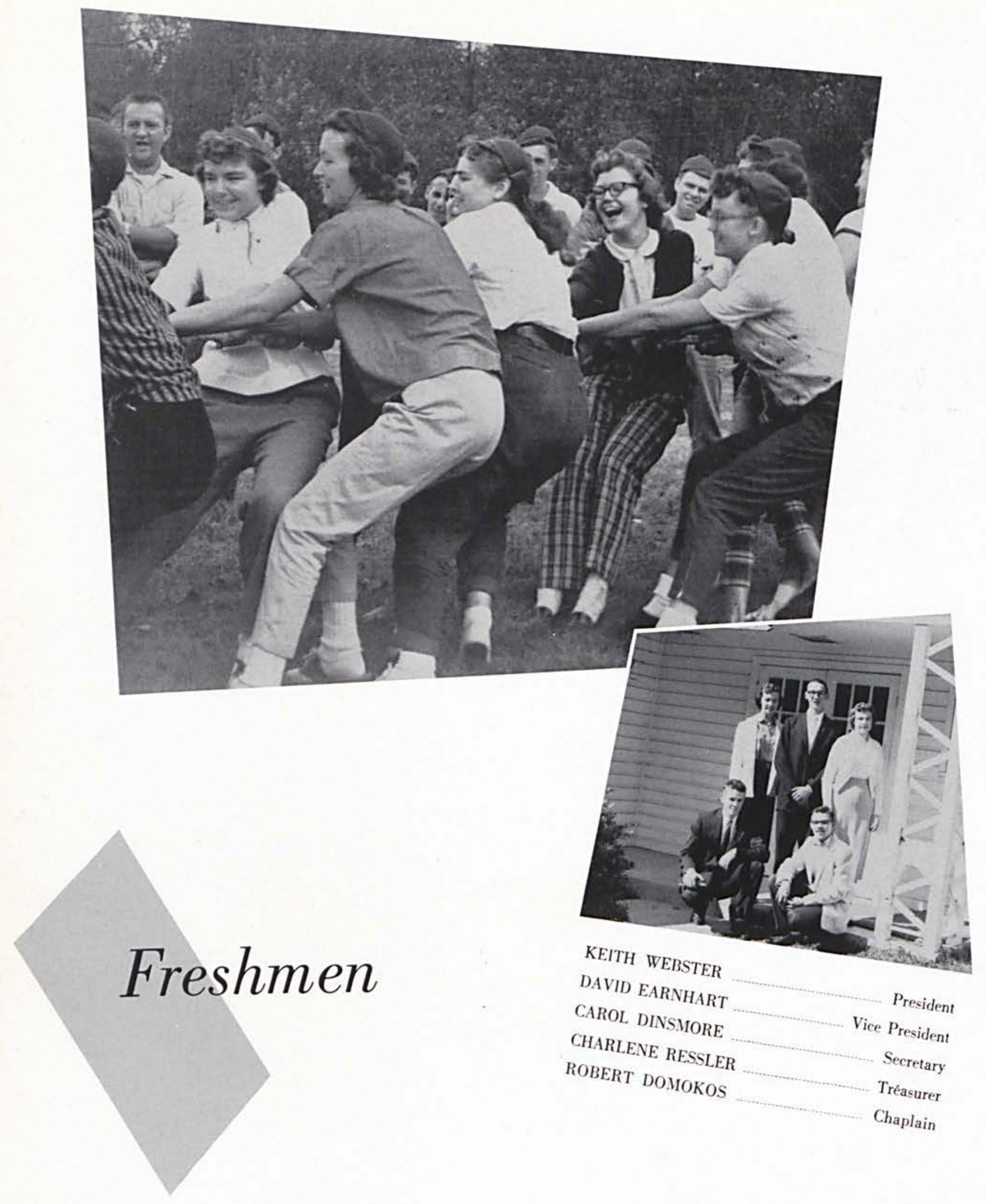

"We enter to learn and go forth to serve." 
DALE ADAMS

Meadville, Pennsylvania Calvary Baptist Church

VANCE ASHLEY

Avon Lake, Ohio

Trinity Baptist Church

LONNIE BELL

Cincinnati, Ohio

Union Baptist Church

ALLEN BIDDLE

Rutland, Ohio

First Baptist Church

ROBERT BLUMENSTOCK

Teaneck, New Jersey

First Baptist Church

STEVE BOALT

Cleveland, Ohio

Cedar Hill Baptist

ROBERT BOORMAN JR.

Portsmouth, Ohio

Temple Baptist Church

MARGARET CANINE

Blanchester, Ohio

First Baptist Church

LORRAINE CARTWRIGHT

Ewington, Ohio

First Baptist Church

ELDONNA COONS

Brownsburg, Indiana

Waveland Baptist Church

JUDY COUSINEAU

Saginaw, Michigan

Grace Baptist Church

VIRGINIA COX

Russell, Iowa

Center Community Church
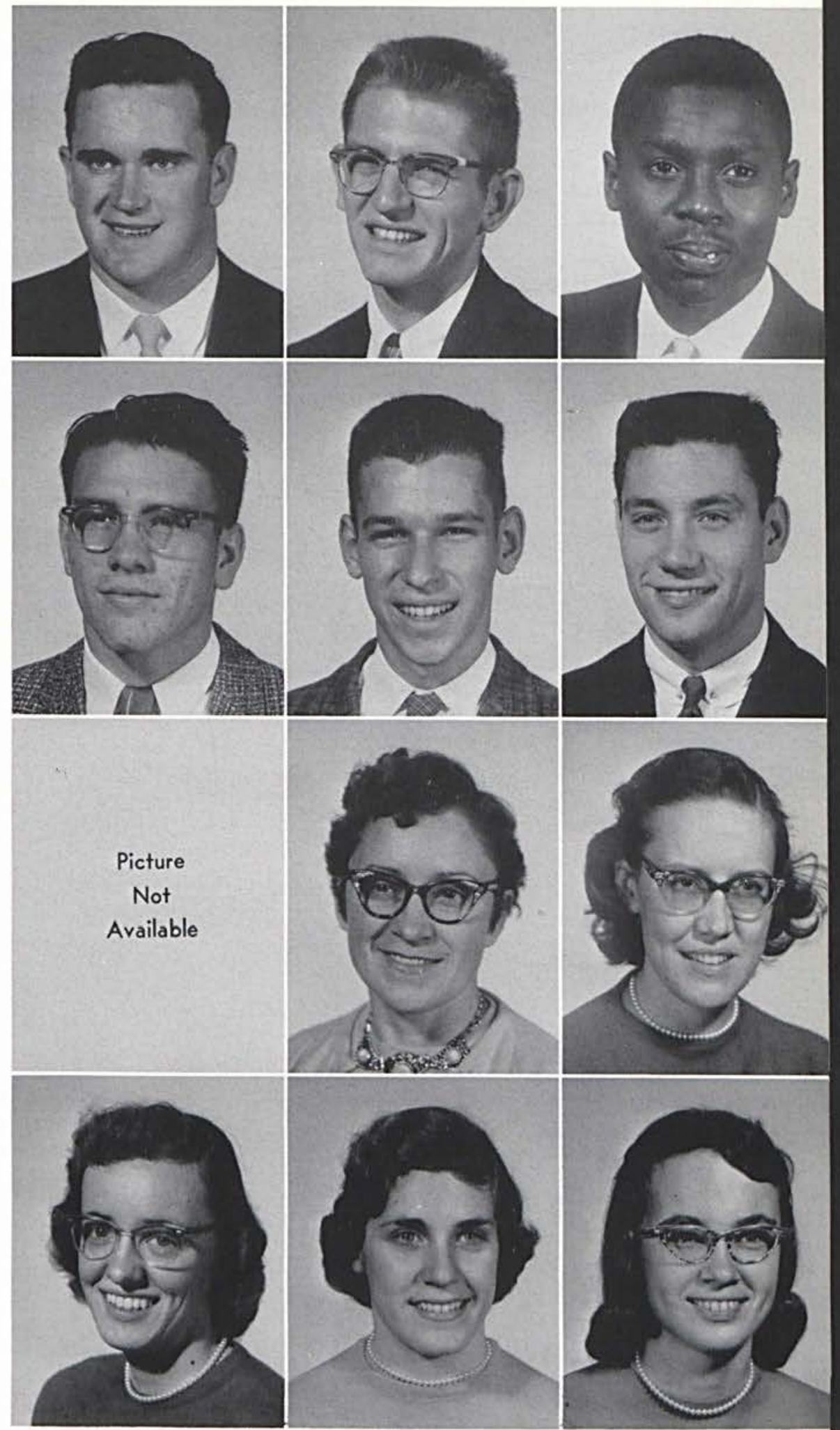

\section{Freshmen}



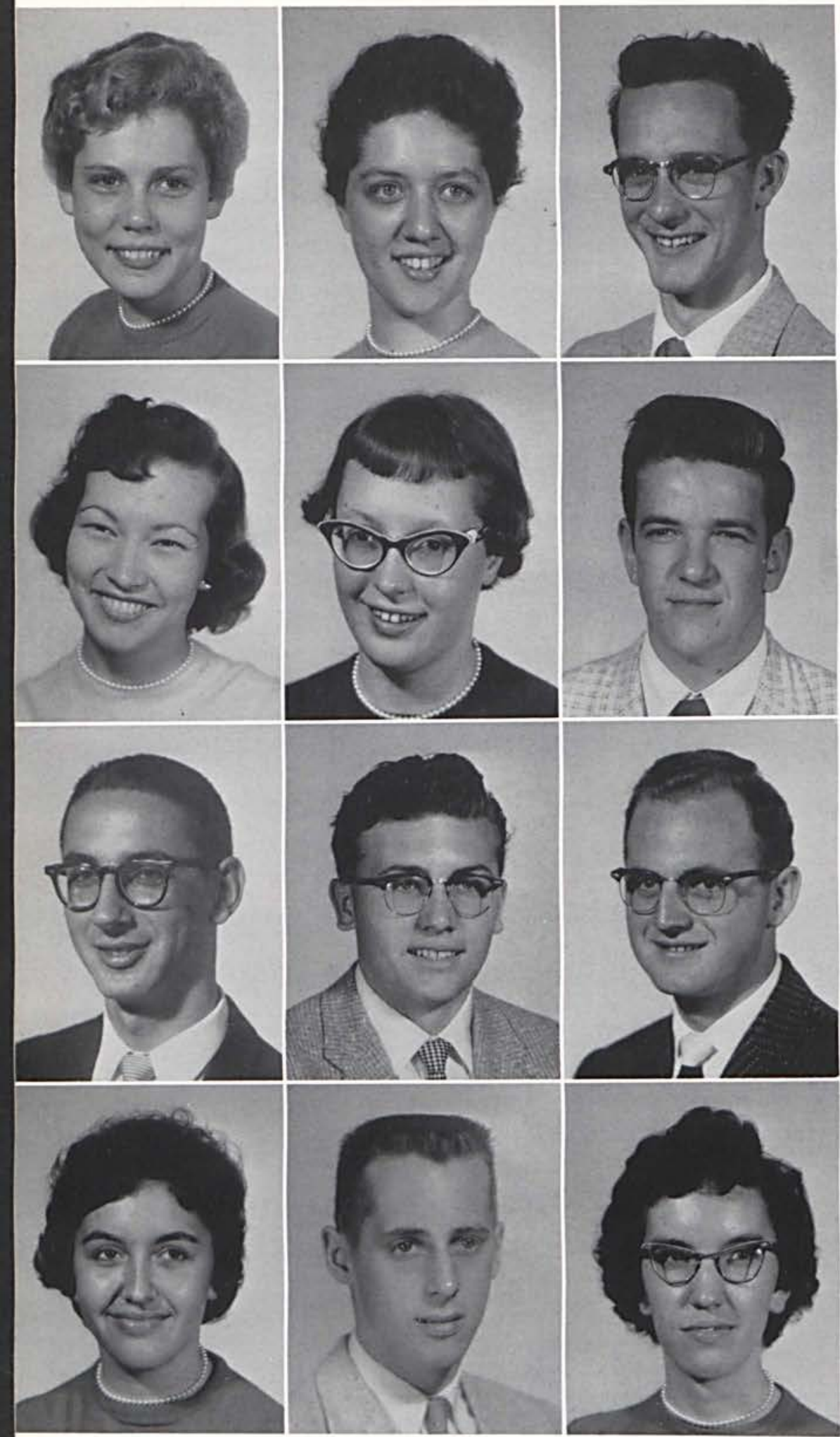
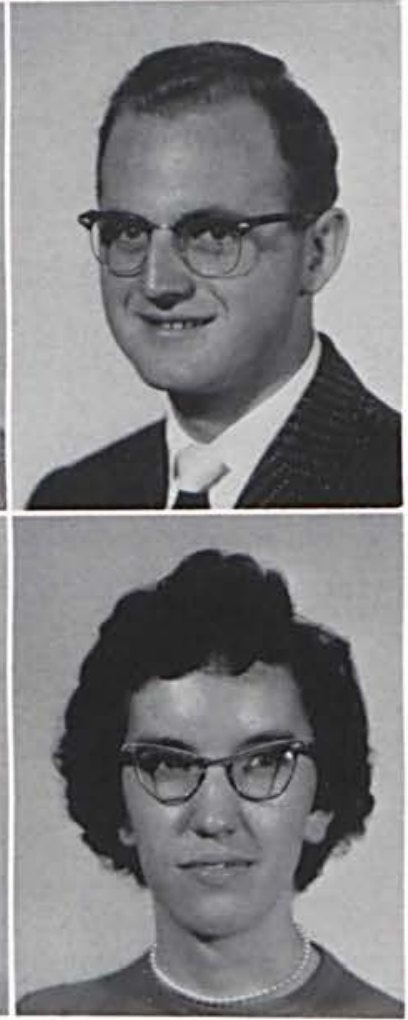

CLAUDIA CROSS

Rochester, Michigan

First Baptist Church

DARLENE DERR

Flint, Michigan

Grace Baptist Church

WAYNE DICKASON

Ortonville, Michigan

Ortonville Baptist Church

CAROL DINSMORE

Cleveland, Ohio

Euclid-Nottingham Baptist Church

SHARON DOBBS

Kuna, Idaho

Kuna Baptist Church

ROBERT DOMOKOS

Windsor, Ohio

Huntsburg Baptist Church

\section{DAVID EARNHART}

Waynesville, Ohio

Xenia Emmanuel Baptist Church

MARVIN FRANK

Hammond, Indiana

Hessville Baptist Church

WALLACE FRANK

Kuna, Idaho

Kuna Baptist Church

SANDRA HAMMERSTROM

North Royalton, Ohio

North Royalton Baptist Church

JAMES HOLCOMB

Paw Paw, Michigan

Community Bible Church

MARGARET HOWARD

Meadville, Pennsylvania

Calvary Baptist Church

\section{Freshmen}


JAMES INMAN

Olean, New York

Christian and Missionary Alliance

DOLLY JESSE

Elyria, Ohio

First Baptist Church

FRED JOHNSON

Cambridge, Ohio

First U.P. Church

\section{RUTH JONES}

Springfield, Ohio

Blessed Hope Baptist Church

ELAINE JORDAN

Richmond, Virginia

Berean Baptist Church

TONY KILBOURN

Hudson, Michigan

Locust Corners Baptist Church

BARBARA KRUTSINGER

Argenta, Illinois

Riverside Baptist Church

KAY LAMB

Crawfordsville, Indiana

East Side Baptist Church

CAROLE LANIUS

Covington, Kentucky

Calvary Baptist Church

DAVID LEWIS

Portsmouth, Ohio

Temple Baptist Church

PAULINE LOWE

Osseo, Michigan

Locust Corners Baptist Church

MARVIN McCASLIN

Lorain, Ohio

Penfield Junction Baptist Church
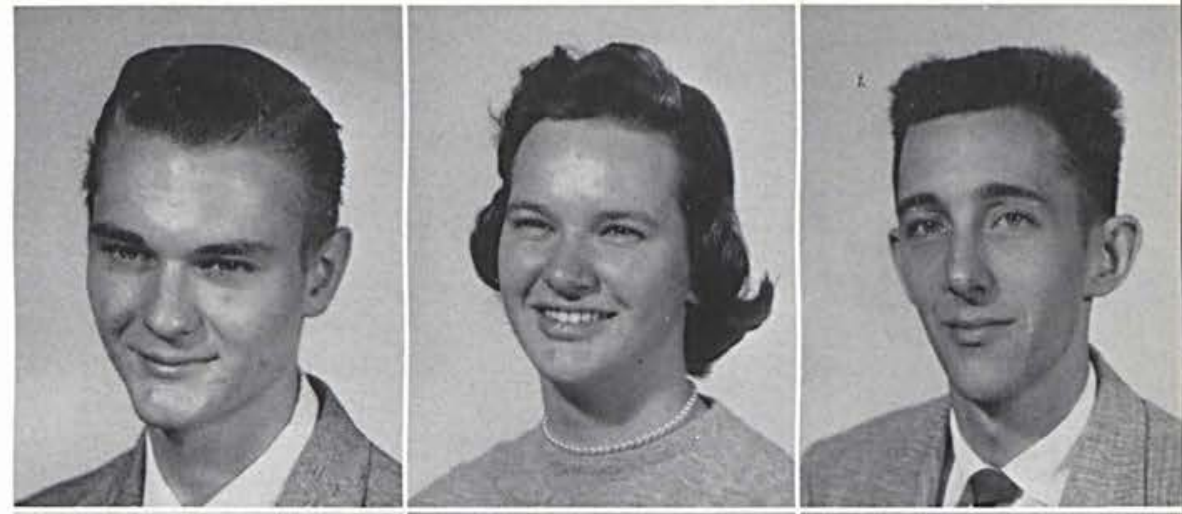

Picture

Not

Available
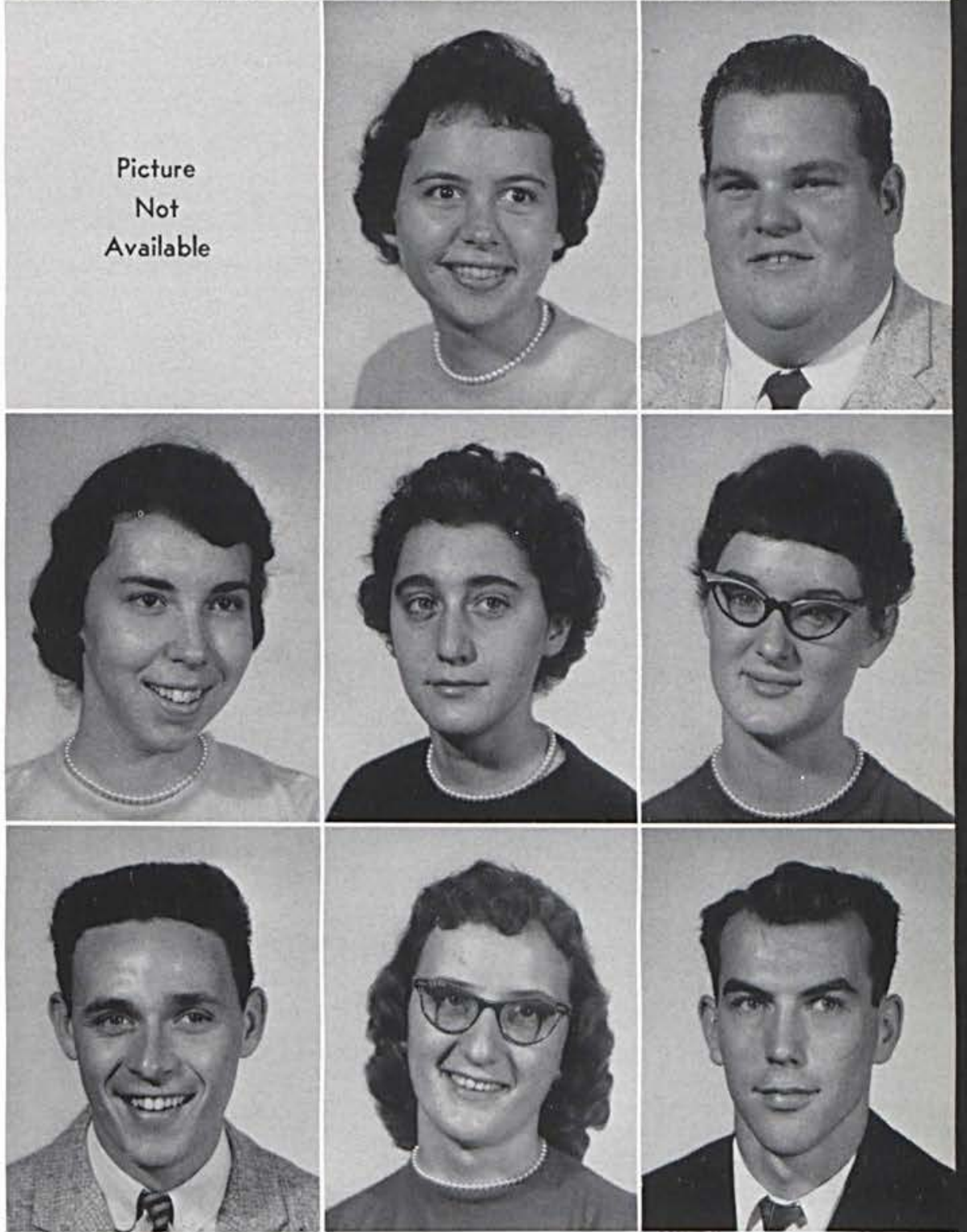
HAROLD RODIN

Burlington, Jowa

Kaimuki Community Church

CATHERINE SHELTON

Clinton. Michigan

Clinton Church

RICHARD SHOFF

Cheektowaga, New York

DAVID SKAGGS

Portsmouth, Ohio

Temple Baptist Church

DAVID SMELSER

Medina, Ohio

First Baptist Church

MARILYN SMITH

Xenia, Ohio

First Rejormed Church

BOGLE STANDIFER

Galion, Ohio

First Baptist Church

DENNIS STILLWELL

Huntsburg, Ohio

Huntsburg Baptist Church

JACK STITT

Altoona, Pennsylvania

Calvary Baptist Church

OTIS STONE

Rochelle, Illinois

First Baptist Church

ESTHER STUTESMAN

Tecumseh, Michigan

Britton Bethel Baptist

CHARLES TAYLOR

Traverse City, Michigan

Immanuel Baptist Church
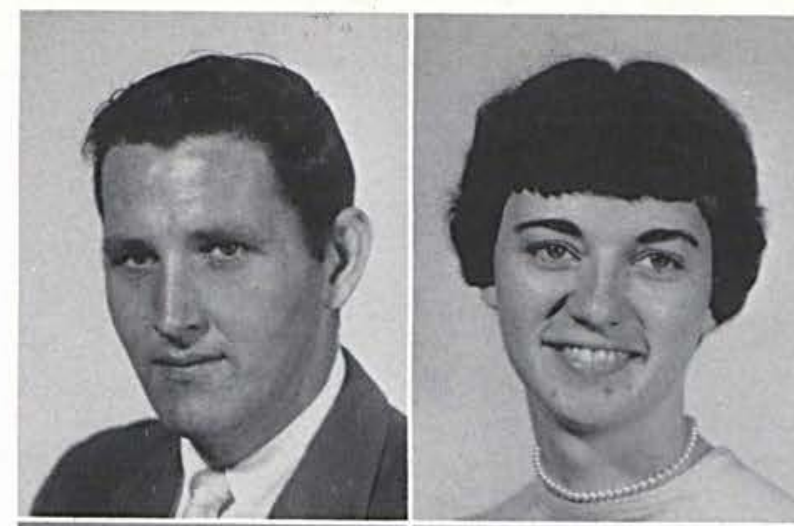

Picture

Not

Available
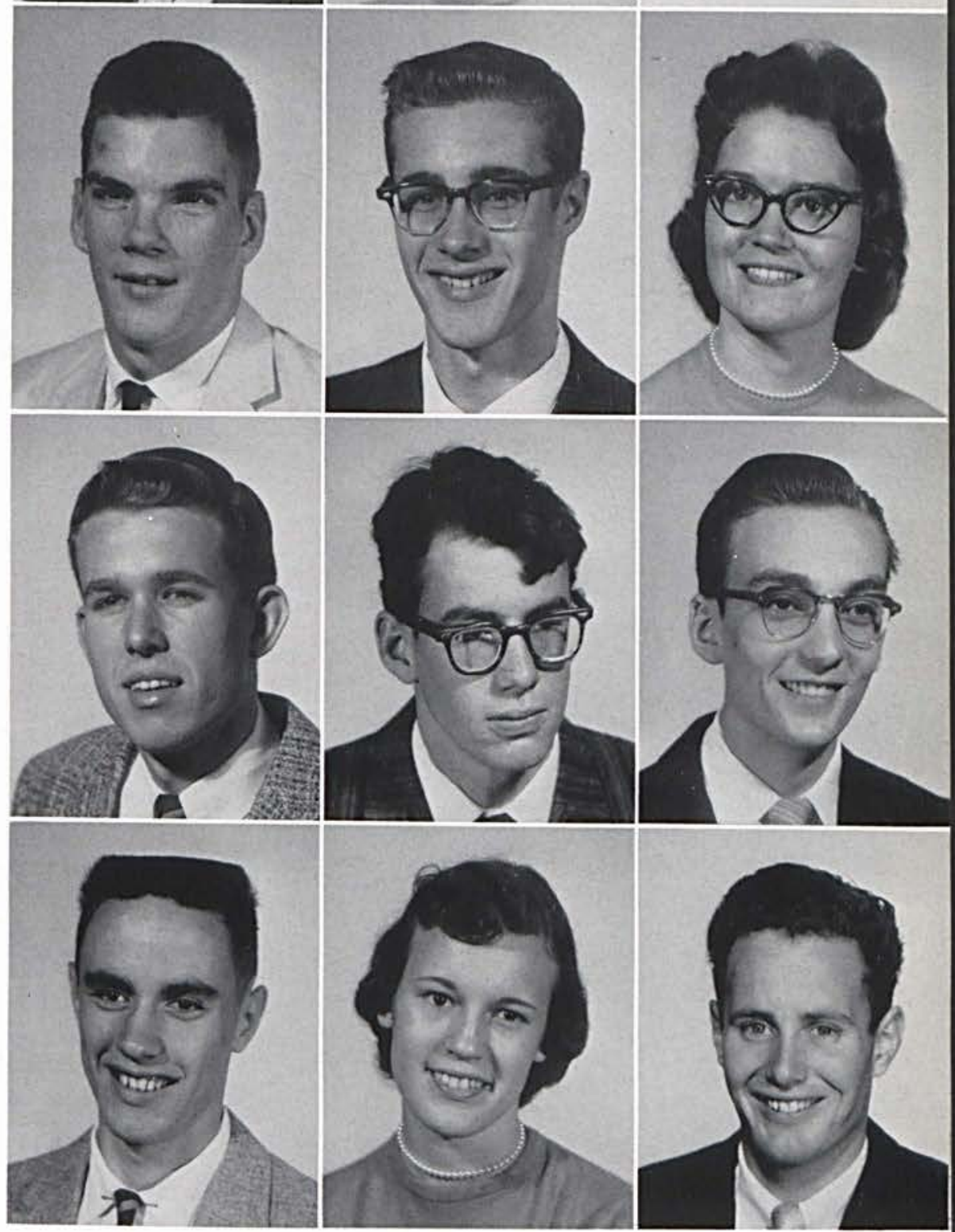


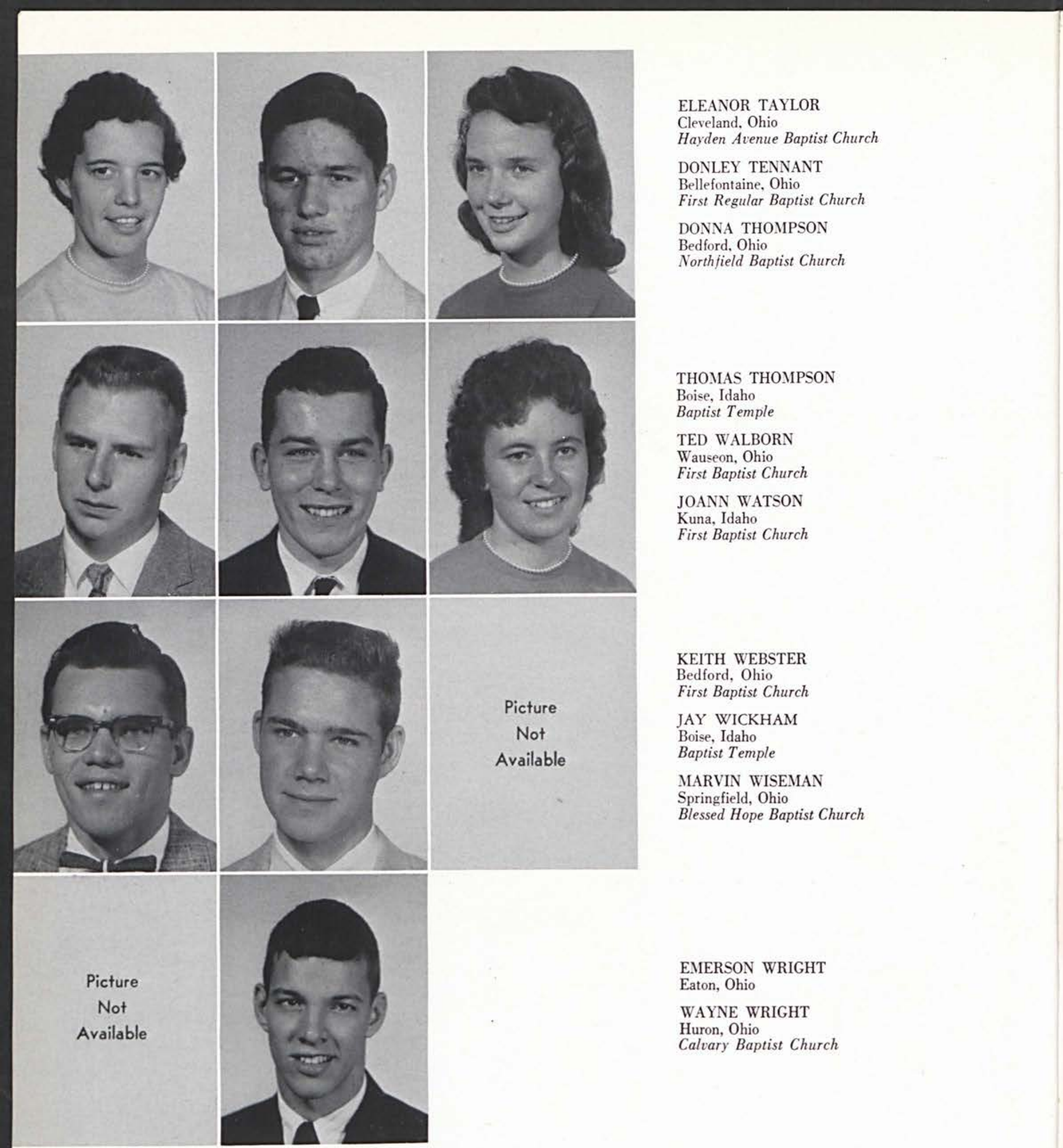

ELEANOR TAYLOR

Cleveland, Ohio

Hayden Avenue Baptist Church

DONLEY TENNANT

Bellefontaine, Ohio

First Regular Baptist Church

DONNA THOMPSON

Bedford, Ohio

Northfield Baptist Church

THOMAS THOMPSON

Boise, Idaho

Baptist Temple

TED WALBORN

Wauseon, Ohio

First Baptist Church

JOANN WATSON

Kuna, Idaho

First Baptist Church

KEITH WEBSTER

Bedford, Ohio

First Baptist Church

JAY WICKHAM

Boise, Idaho

Baptist Temple

MARVIN WISEMAN

Springfield, Ohio

Blessed Hope Baptist Church

EMERSON WRIGHT

Eaton, Ohio

WAYNE WRIGHT

Huron, Ohio

Calvary Baptist Church 


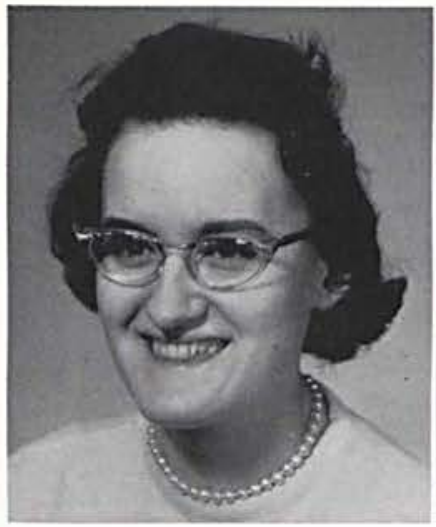

JEAN LOCKERBIE

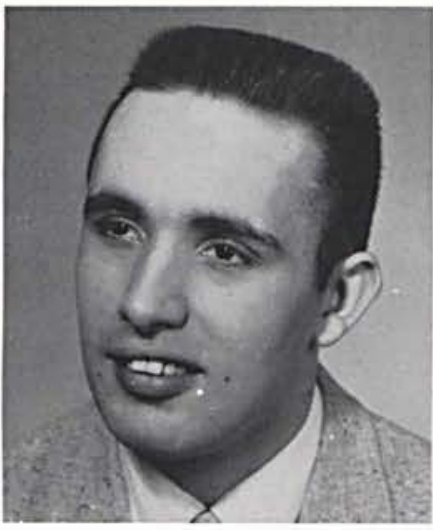

GARY LUSCOMBE

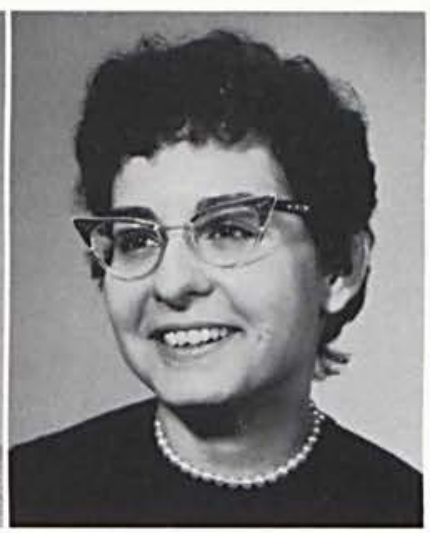

BEVERLY LYON

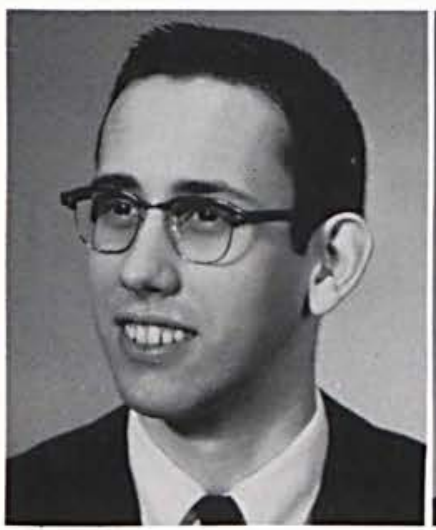

LOREN SCHENCK

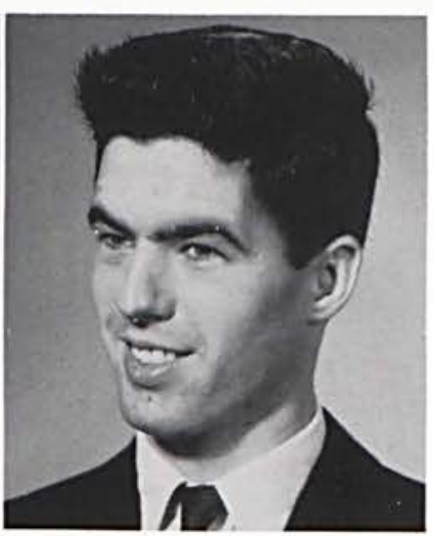

WAYNE STANCHFIELD
Second Semester Students

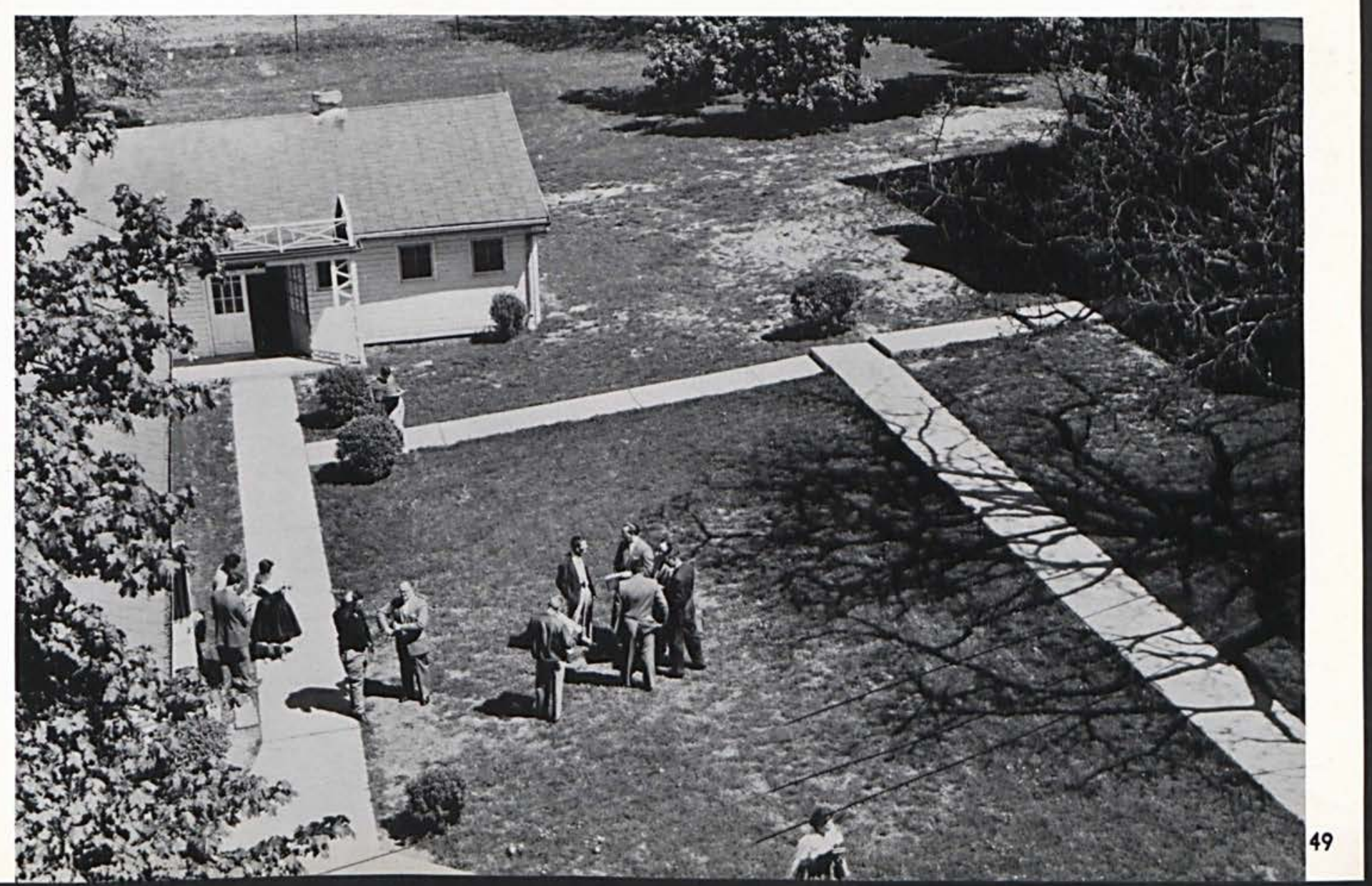




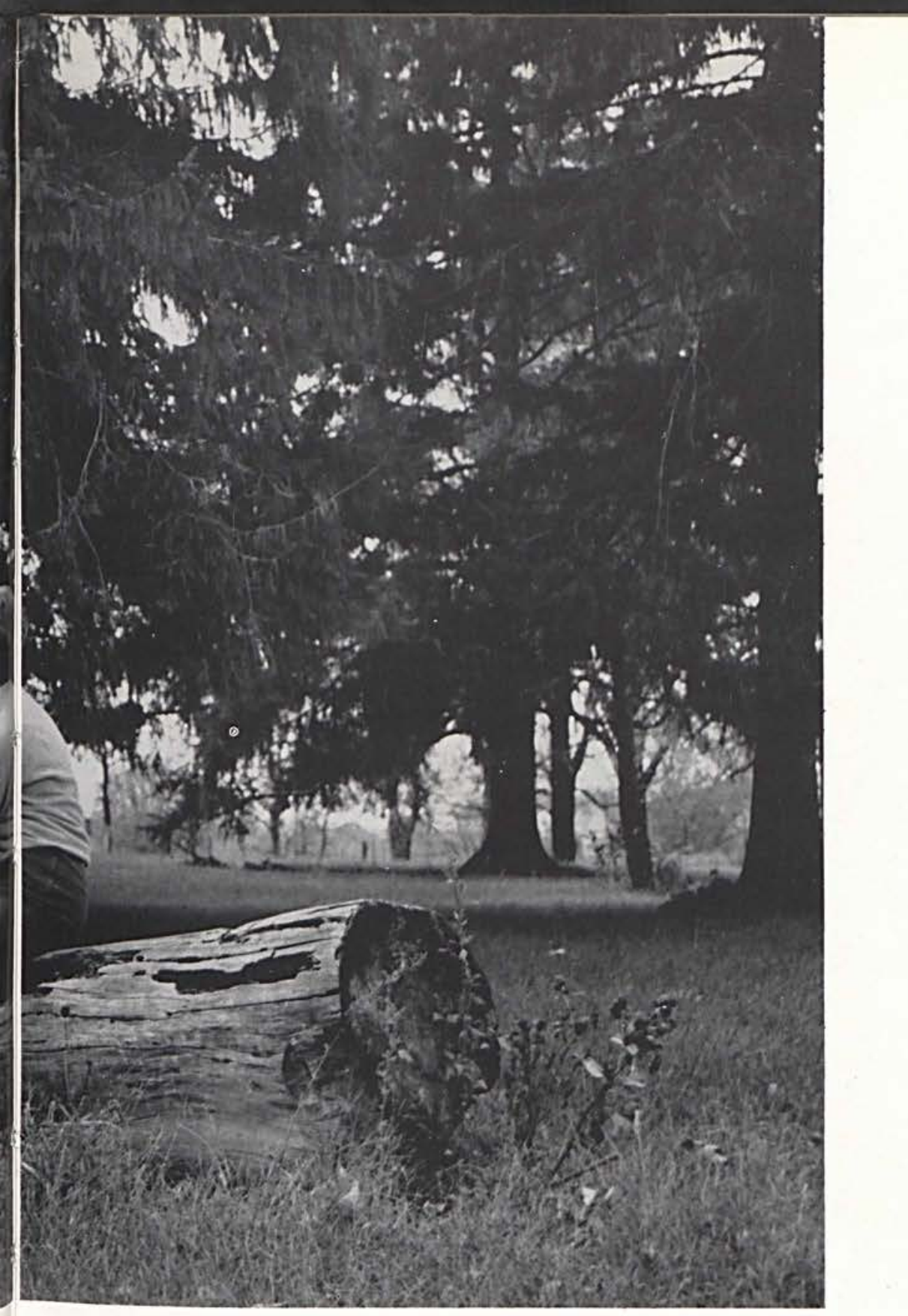




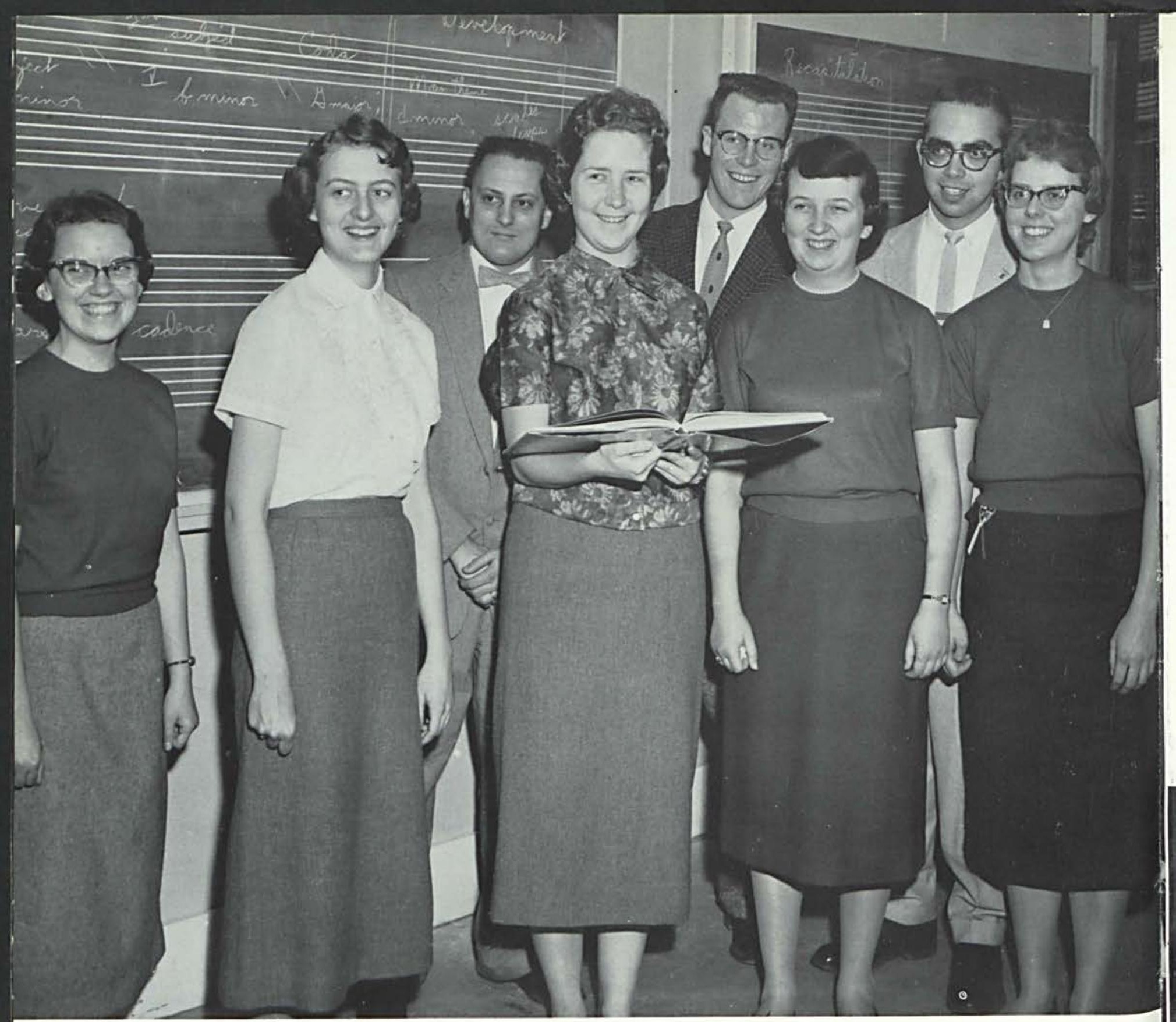

LEFT TO RIGHT:Ruth Yost, Lois Jeremiah, James Neely, Shirley Harrington, Dwayne Frank, Grace Willetts, Dave Matson, Carol Zoellner.

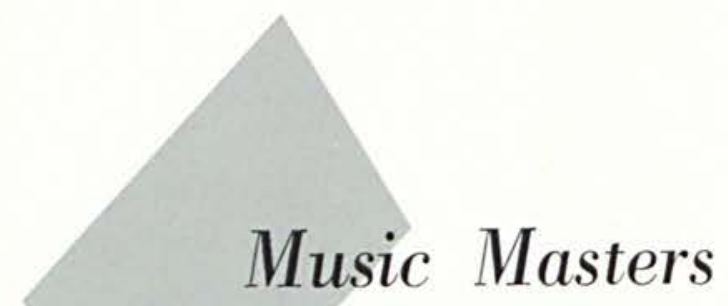

Each year students, outstanding in leadership, cooperation, character, scholarship and service, are chosen for membership in the Modern Music Masters, National Honor Societies. 


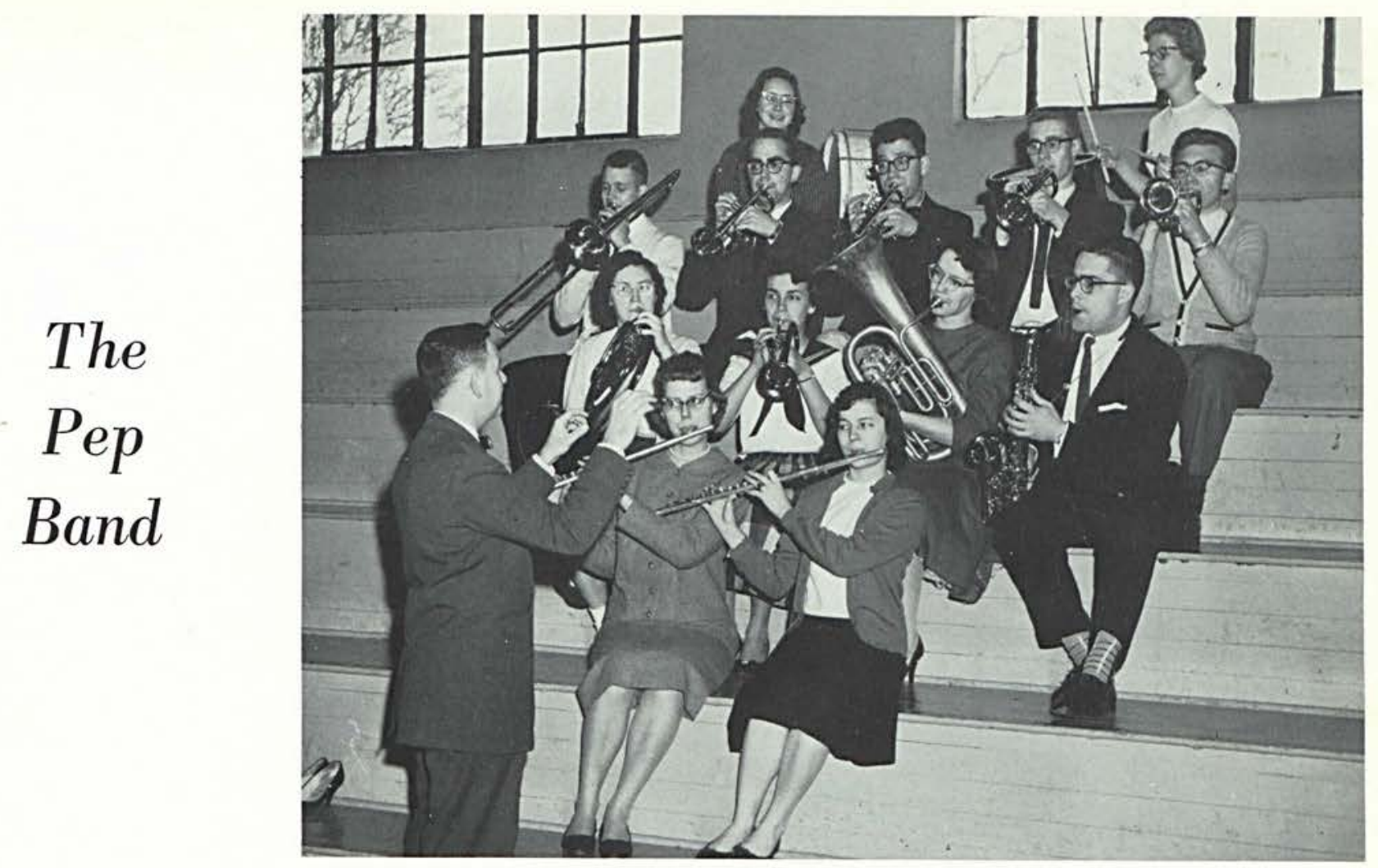

BACK ROW: Carmen Manchester, Sally Roudybush. THIRD ROW: Keith Collett, Dave Matson, Steve Boalt, Dave Smelser, Marvin Frank. SECOND ROW: Joyce Grant, Esther Weiss, Rosemary Smith, Paul Van Kleek. FIRST ROW: Carol Zoellner, Maurine Larsen. Director: Mr. Warren Webber.

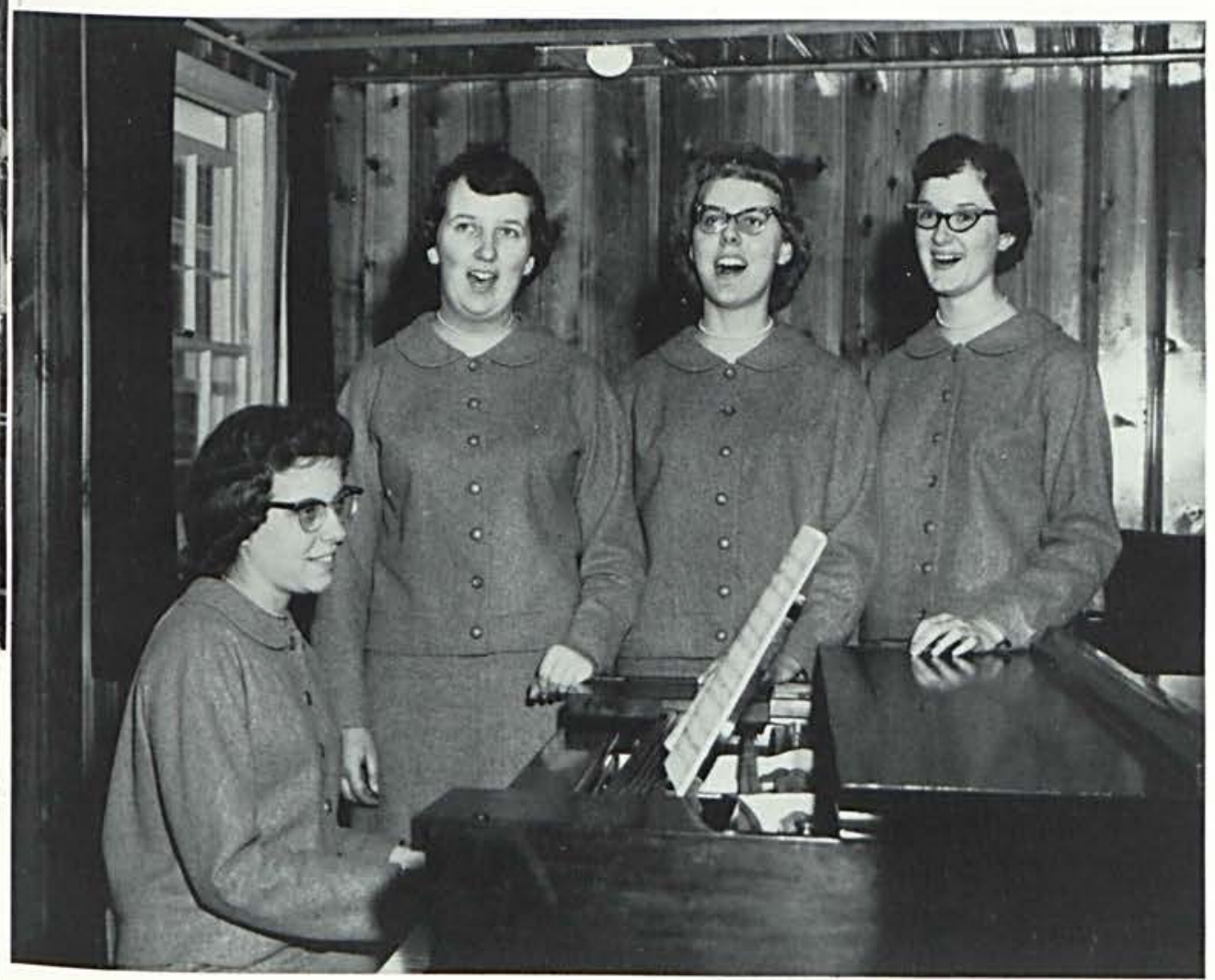

\section{The Ladies' Trio}

Grace Willetts, Carol Zoellner, Carole Lanius. Accompanist: Carolyn Hale. 


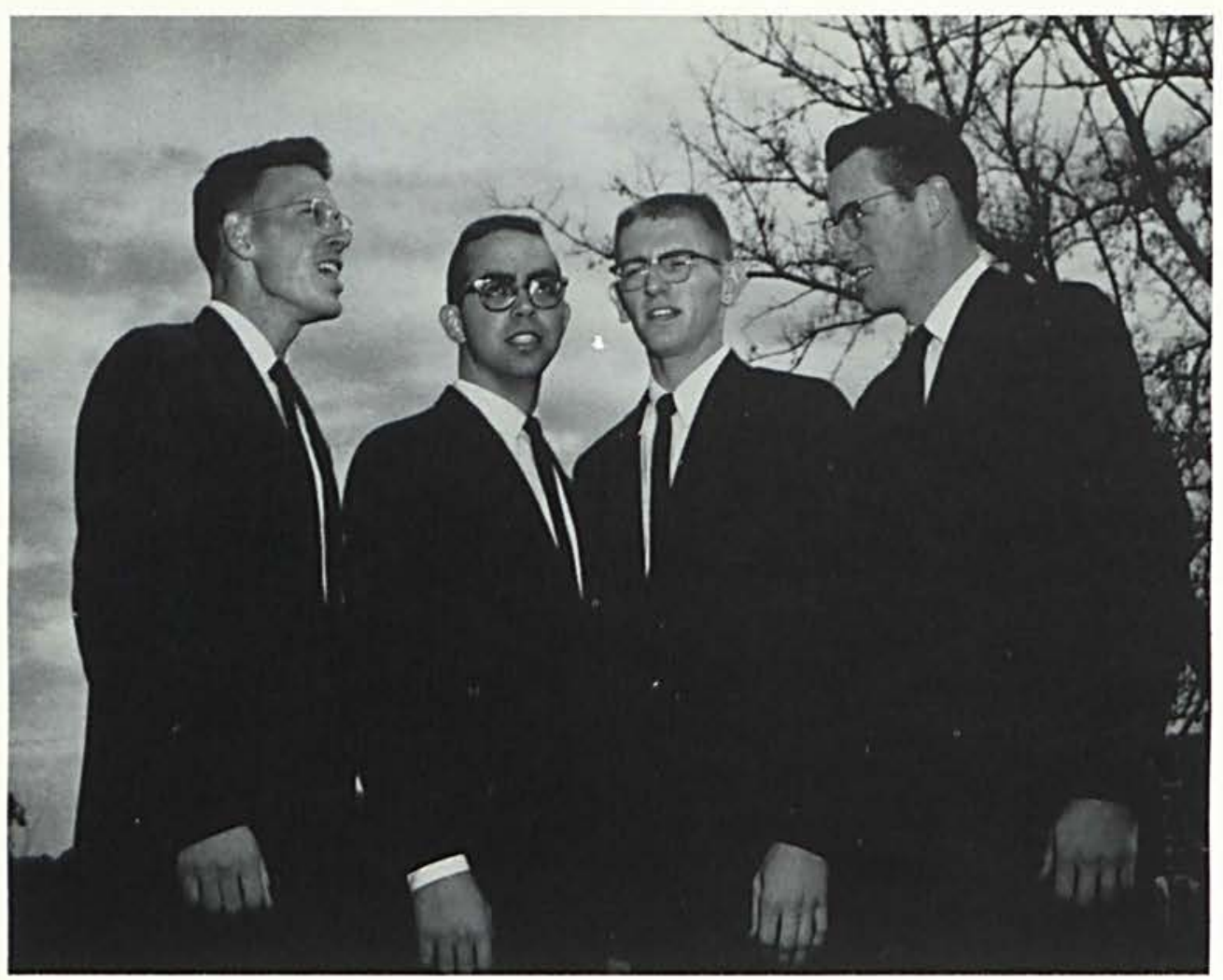

John Butler, Dave Matson, Vance Ashley, Dwayne Frank

\section{The Men's Quartets}

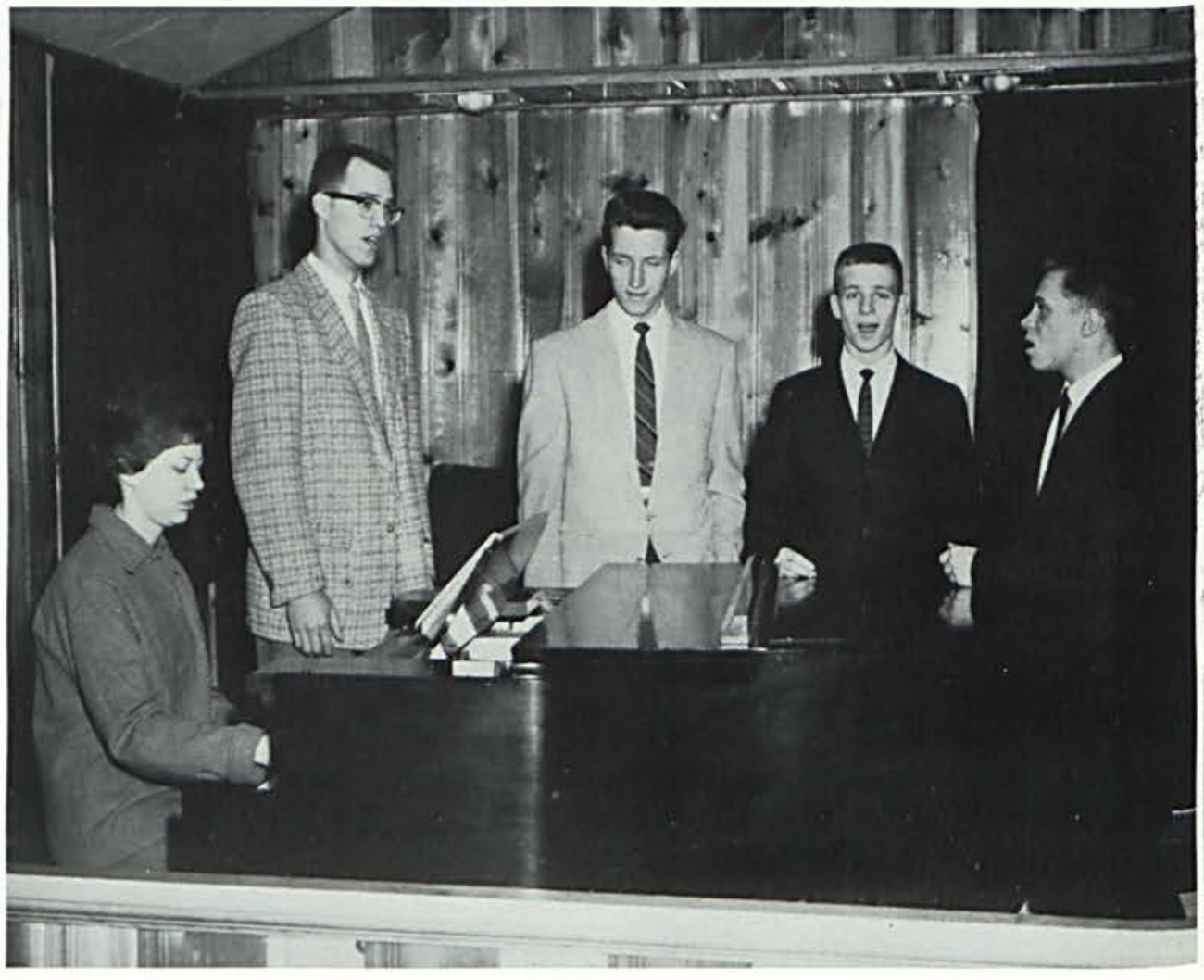

Roy Carr, Warren Woodard, Keith Collett, Dick Cook. Accompanist: Marcia Crothers. 


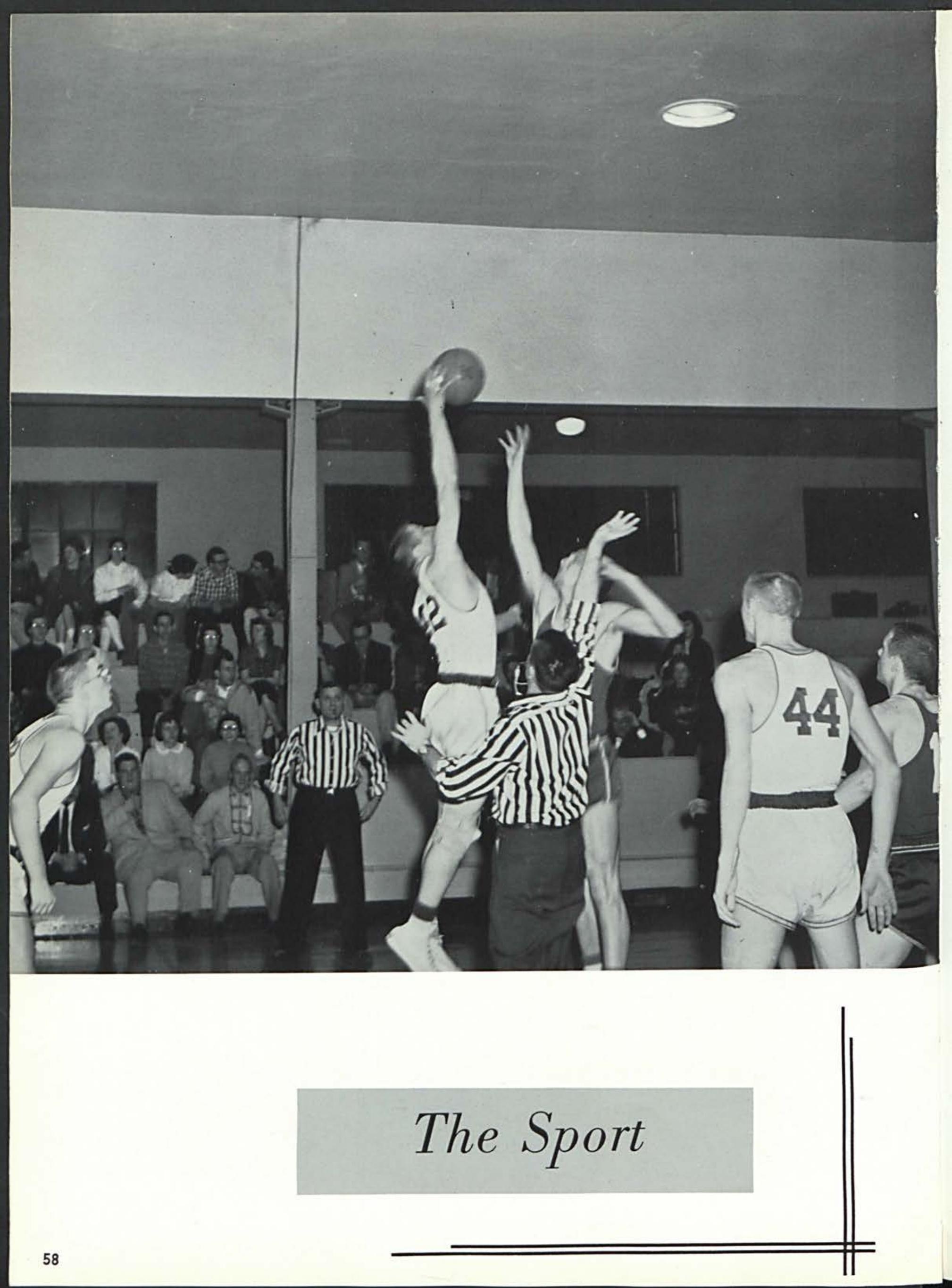



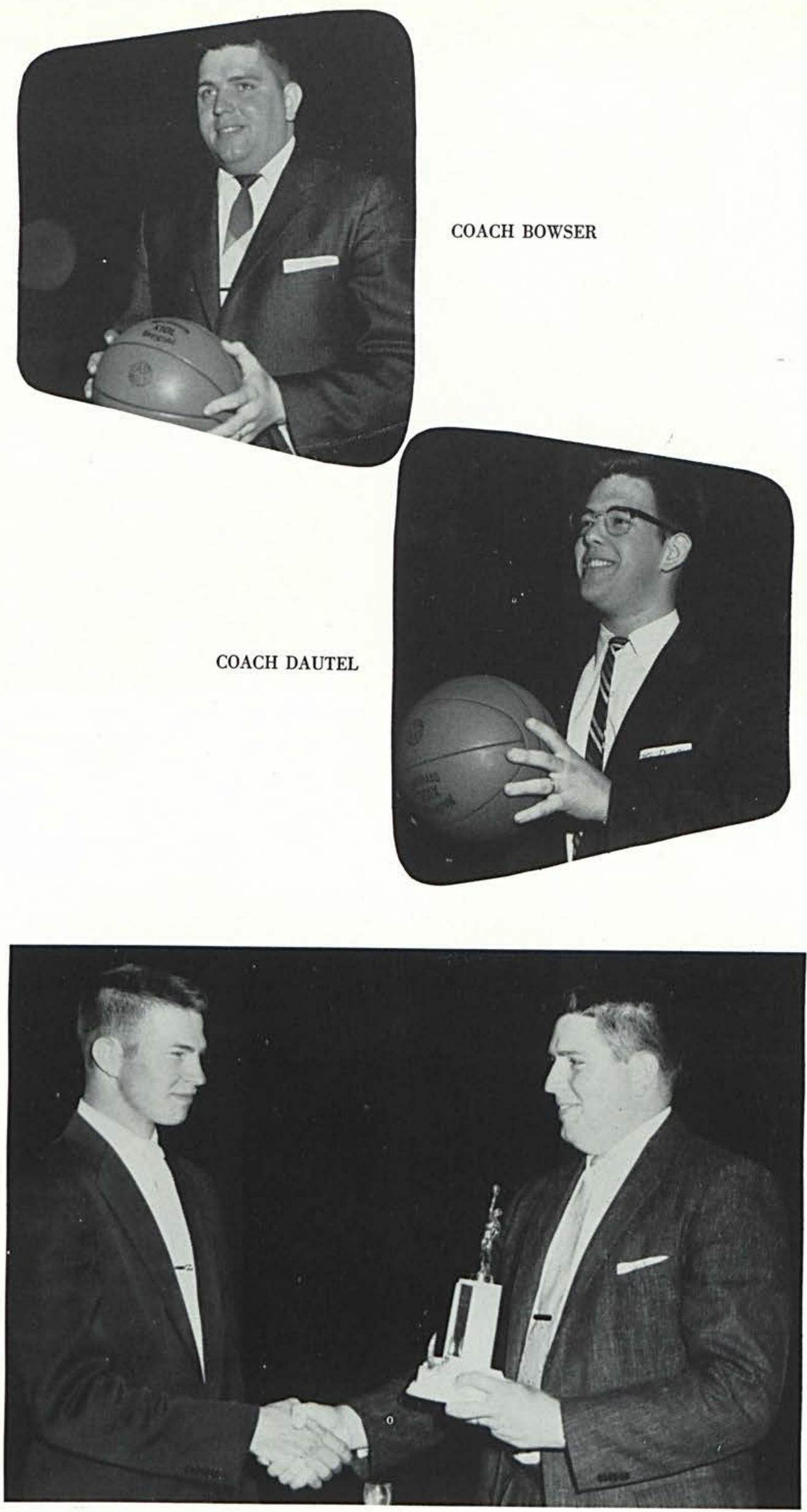

Pete Reese receiving 1957-58 M.V.P. award. 


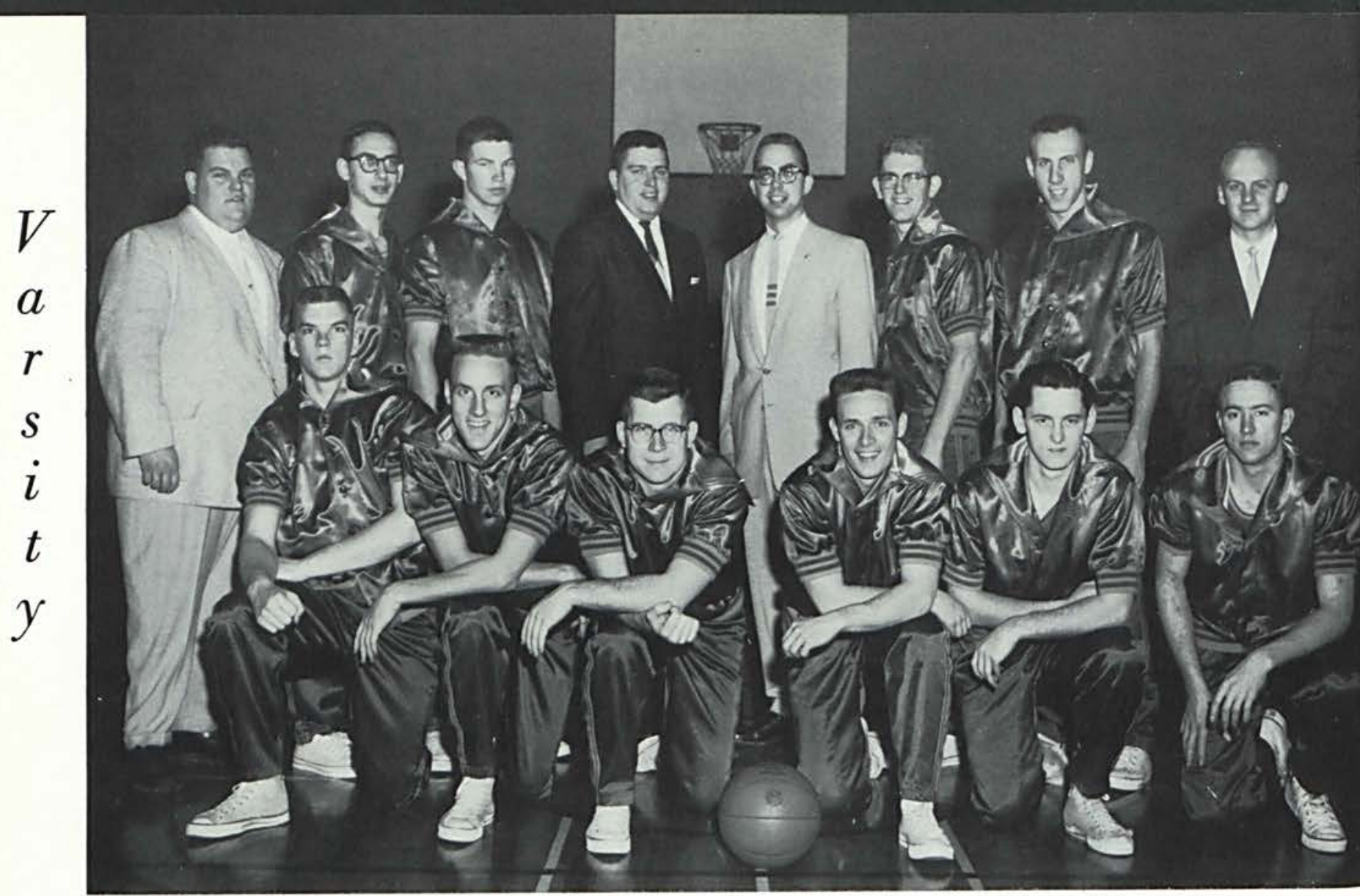

BACK ROW:Tony Kilbourn, Trainer; Dave Earnhart, Pete Reese, Coach Bowser, Dave Matson, Publicity Director; Vance Ashley, John Entner, Dave Thomas, Manager. FRONT ROW: Dave Skaggs, Jim Entner, Bob Humphreys, Dave Lewis, Merlin Ager, Terry Zerby.

\section{Schedule 1958-59}

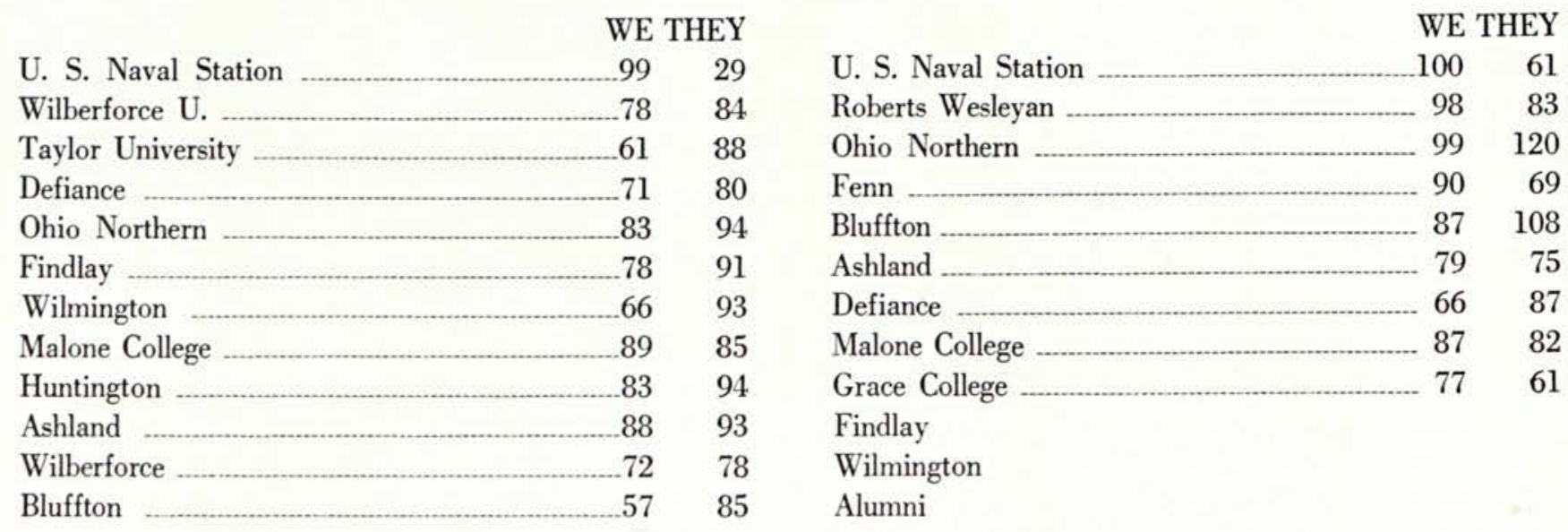



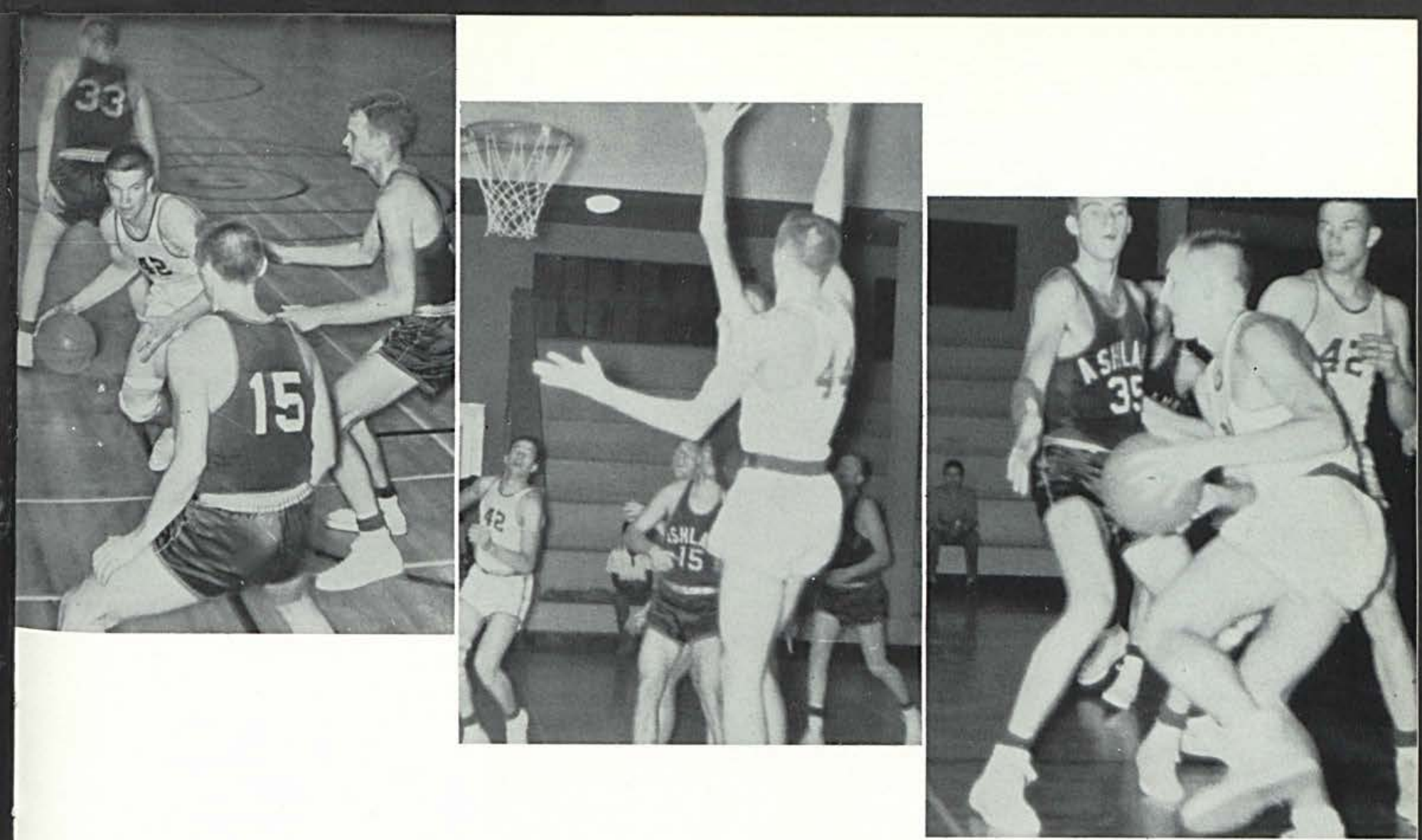

BACK ROW:Dave Earnhart, John Butler, Coach Dautel, Bob Howder, Vance Ashley. FRONT ROW: Otis Stone, Marvin McCaslin, Merlin Ager.

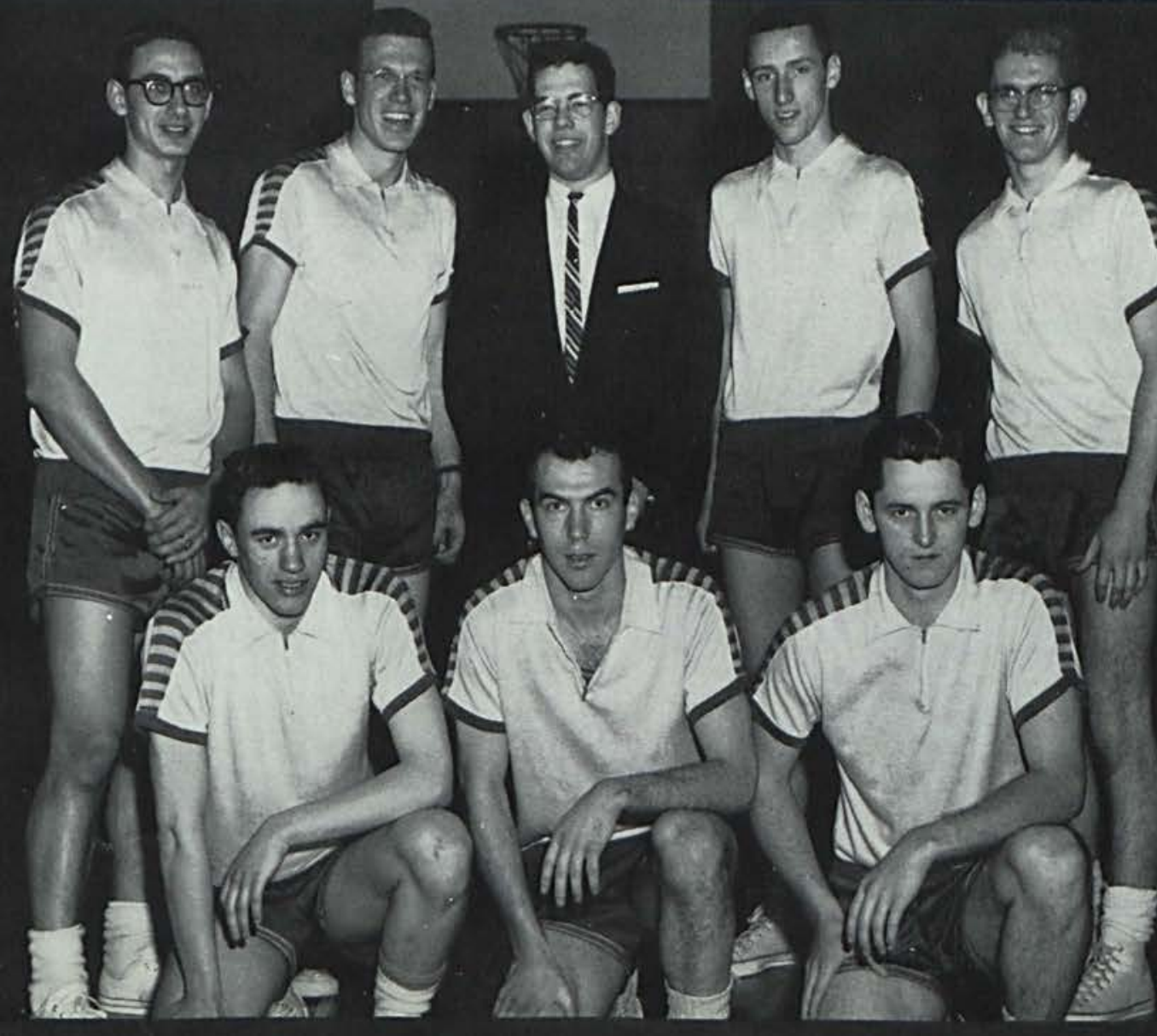




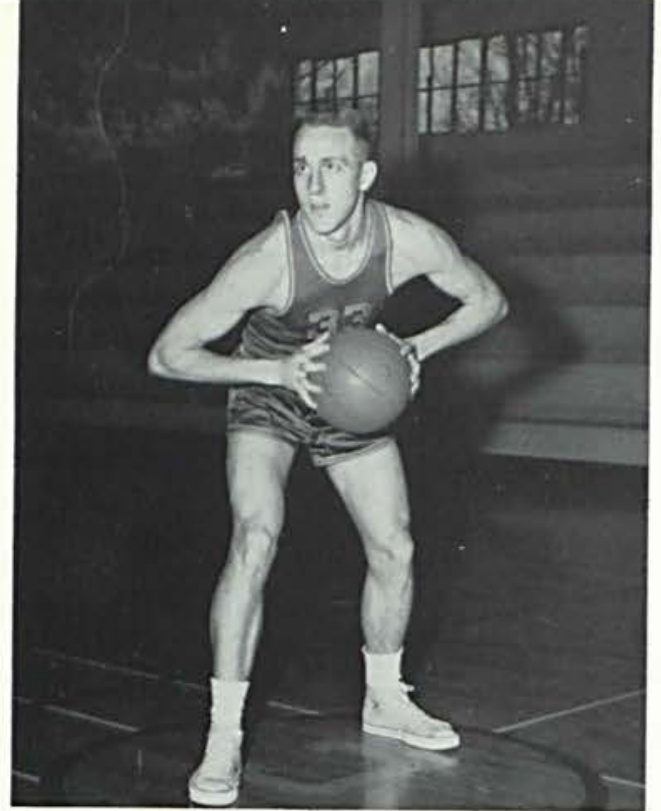

JIM ENTNER

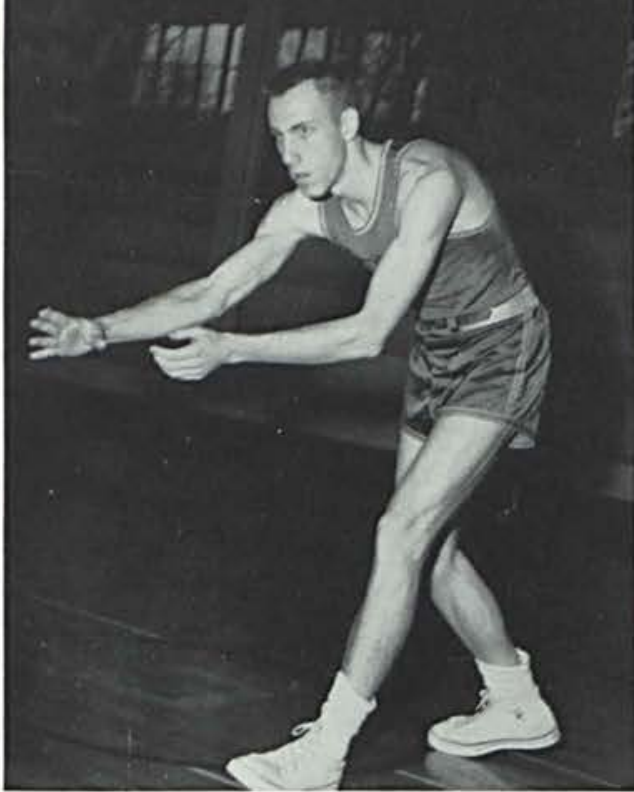

JOHN ENTNER
PETE REESE

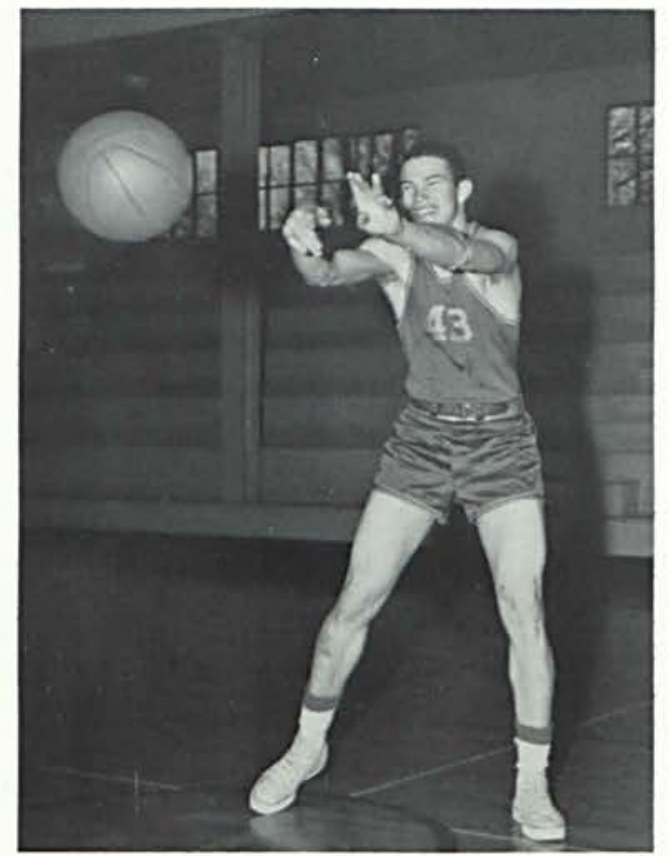

DAVE LEWIS

DAVE SKAGGS

The
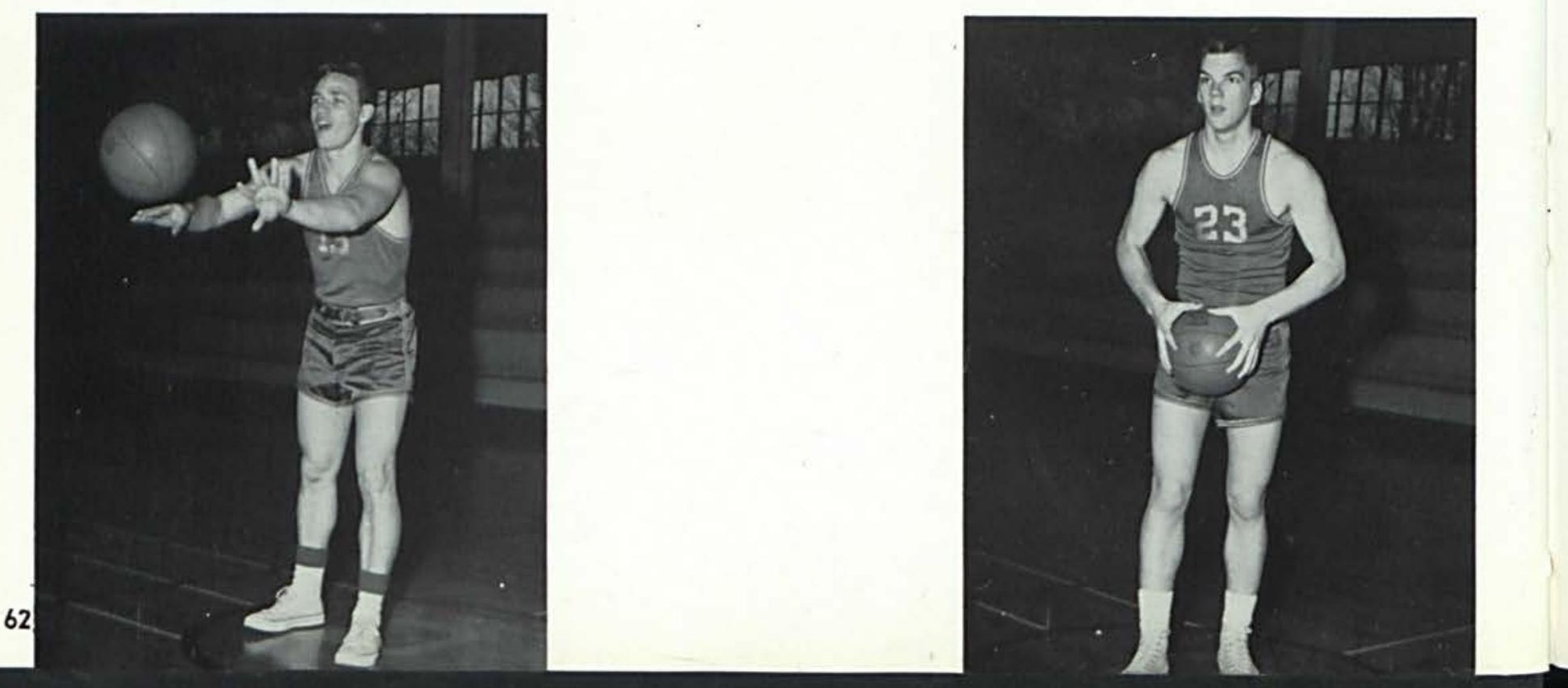
Esther Weiss, Donna Thompson, Sandy Hammerstrom, Sandy Entner
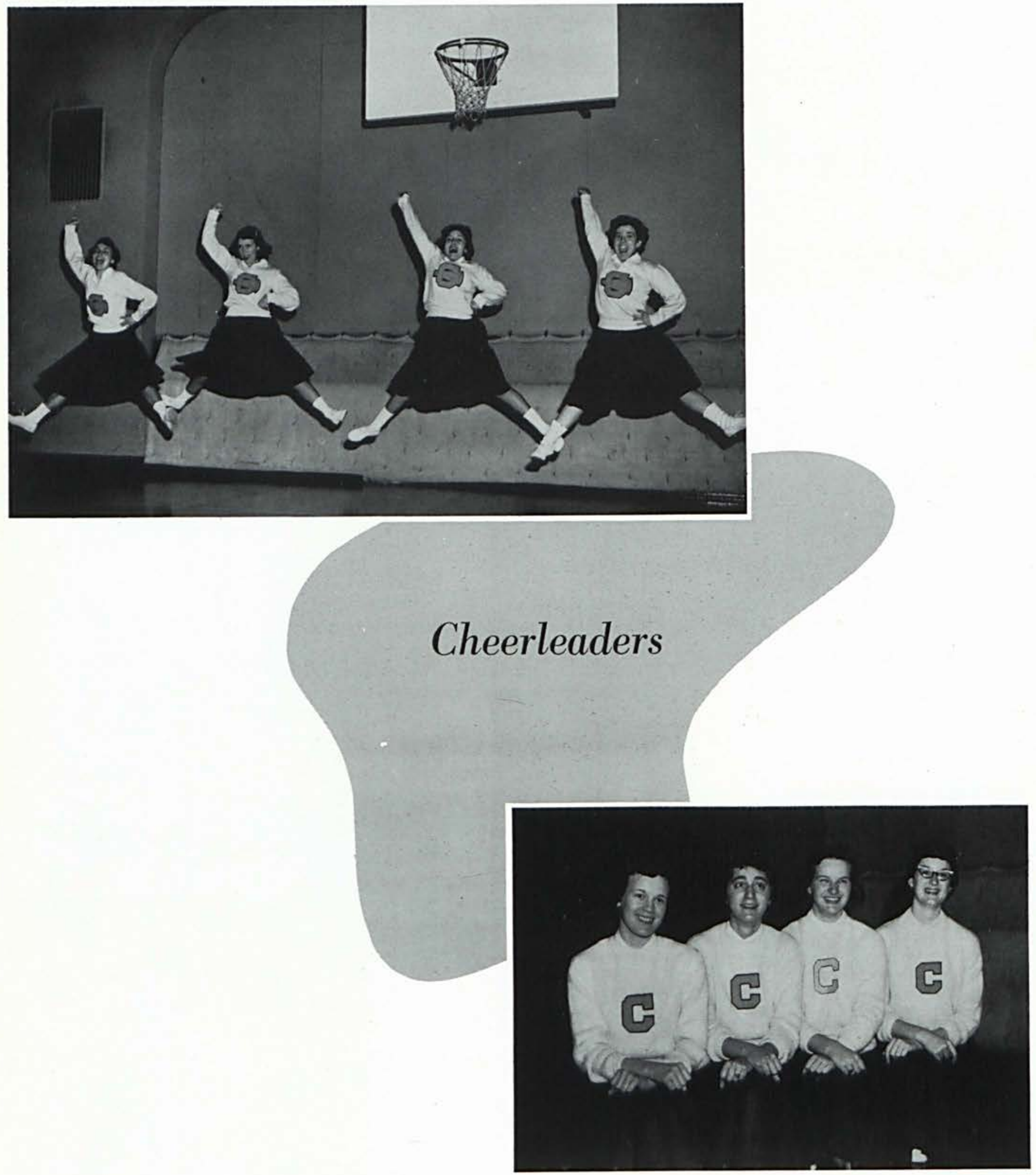

Esther Stutesman, Kay Lamb, Dolly Jesse, Carole Lanius 


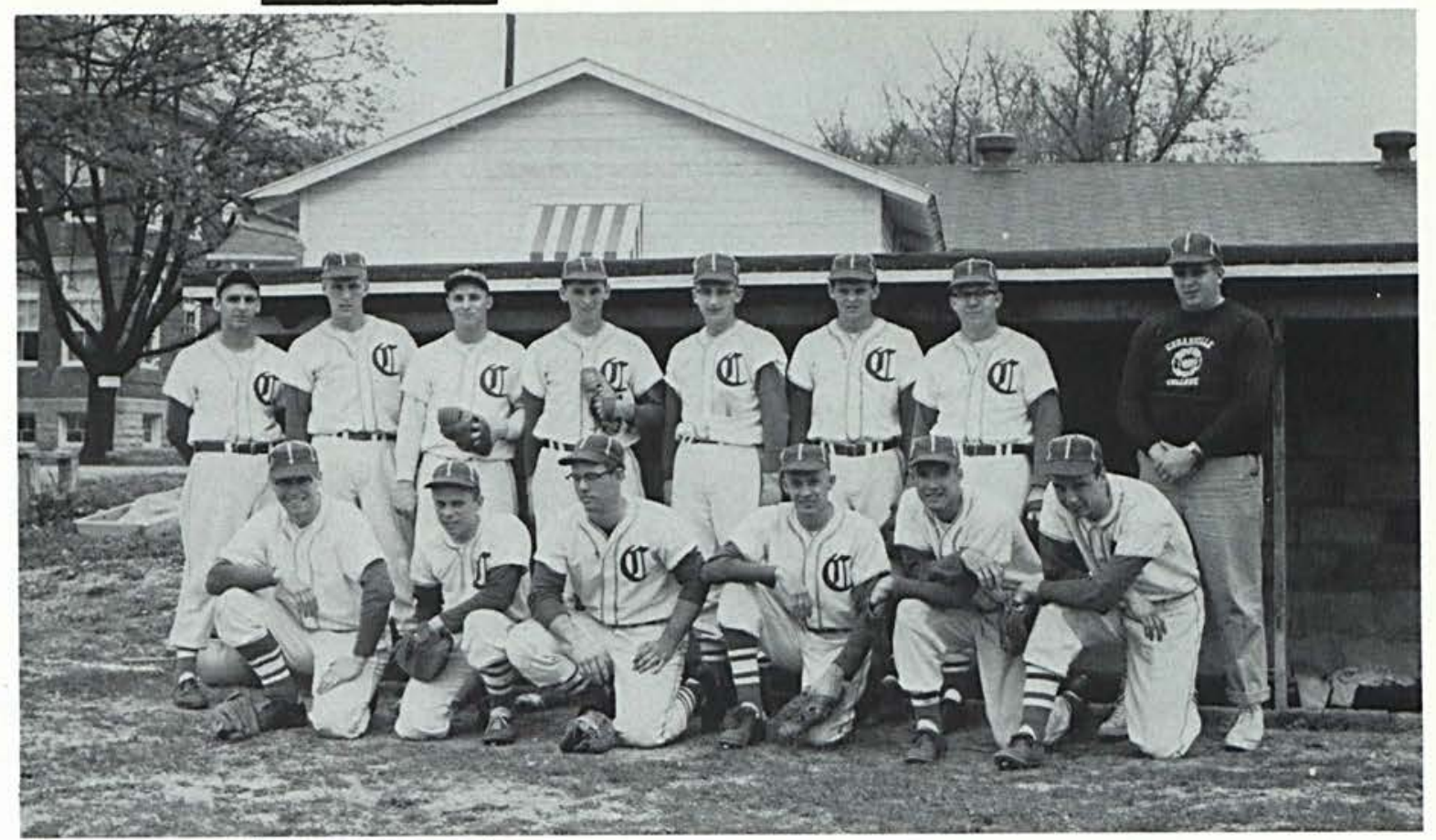

The Yellowjacket Baseball Team

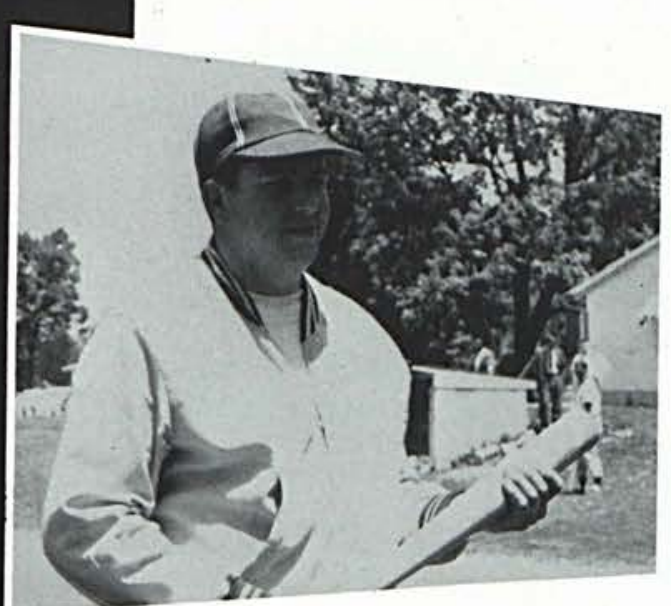

COACH BOWSER

\section{Baseball}

The 1958 Yellowjacket baseball team found the going hard during the season, winning one and losing fourteen. The loss of several players at the beginning of the season handicapped their efforts. Most of this year's team are returning next year and with the prospect of new talent better things should be in store for the Jackets. 


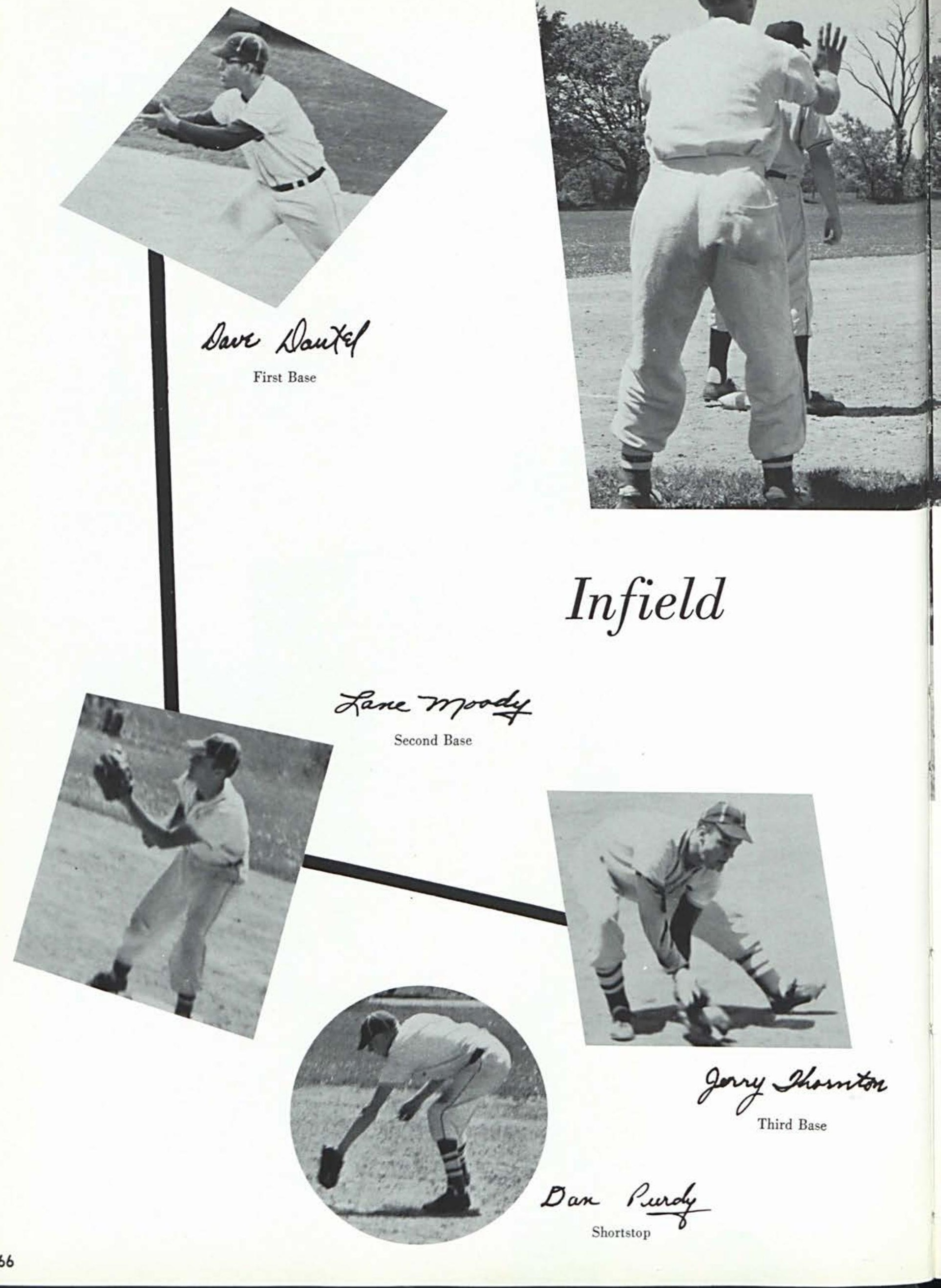



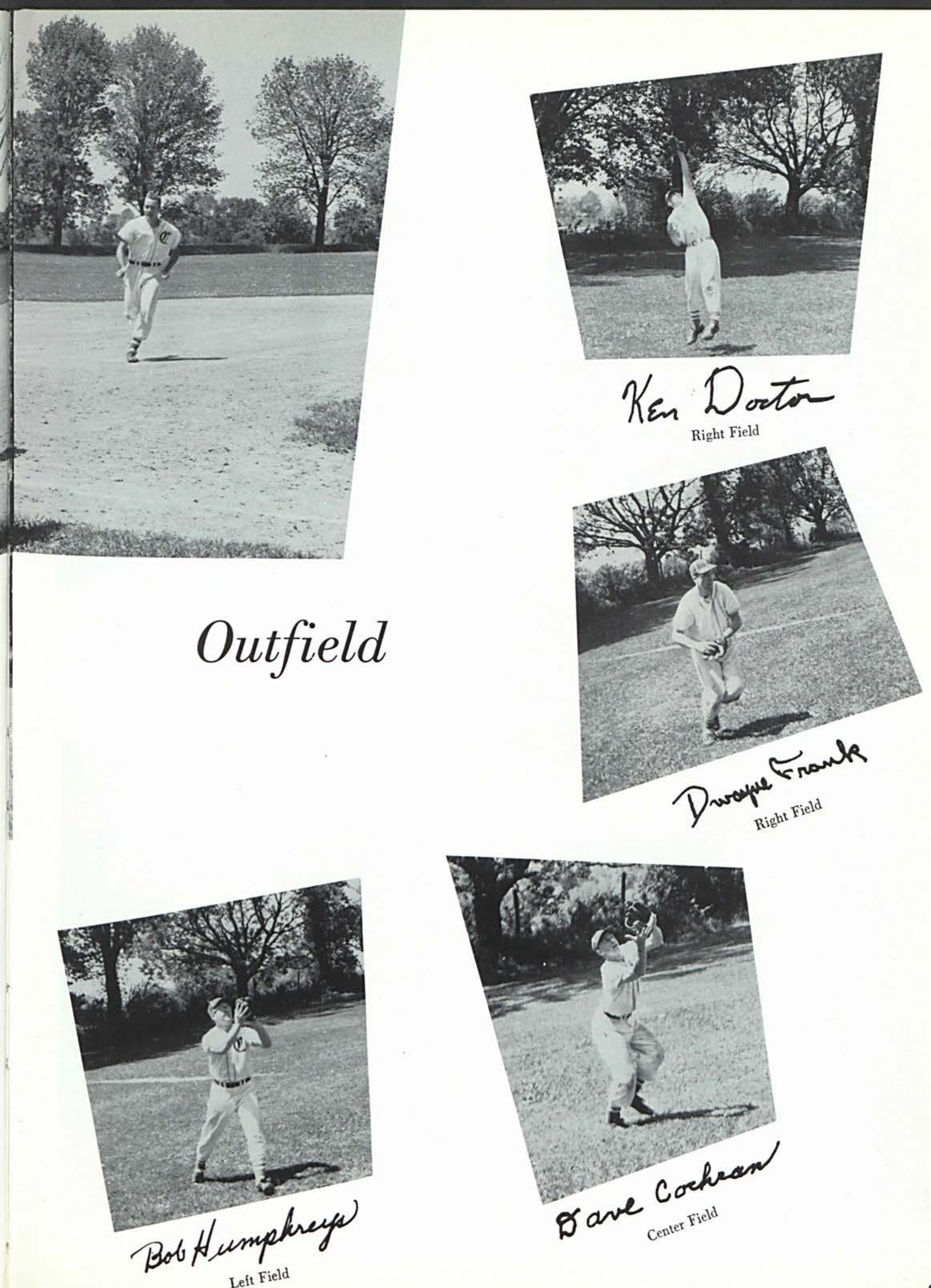


\section{Battery}
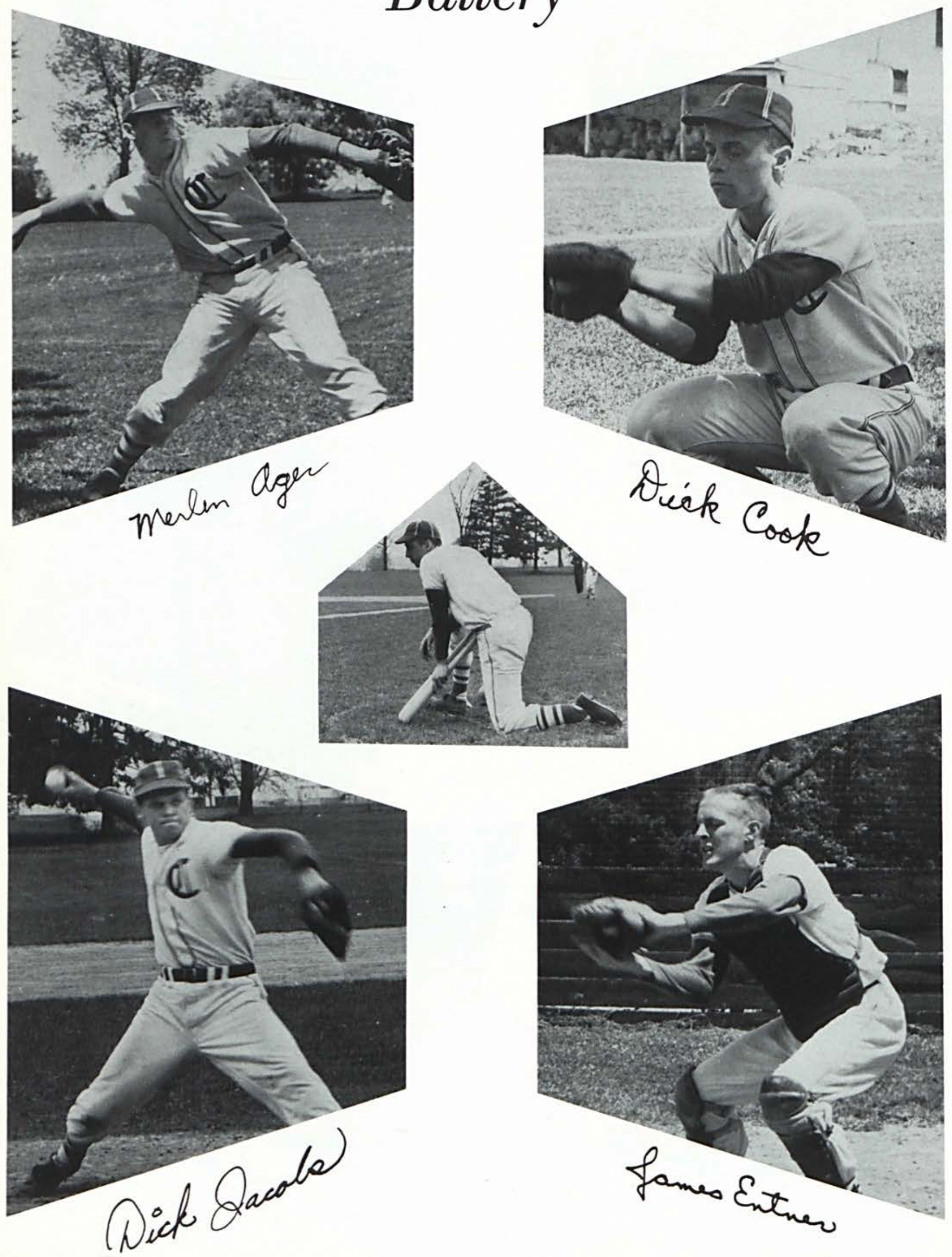


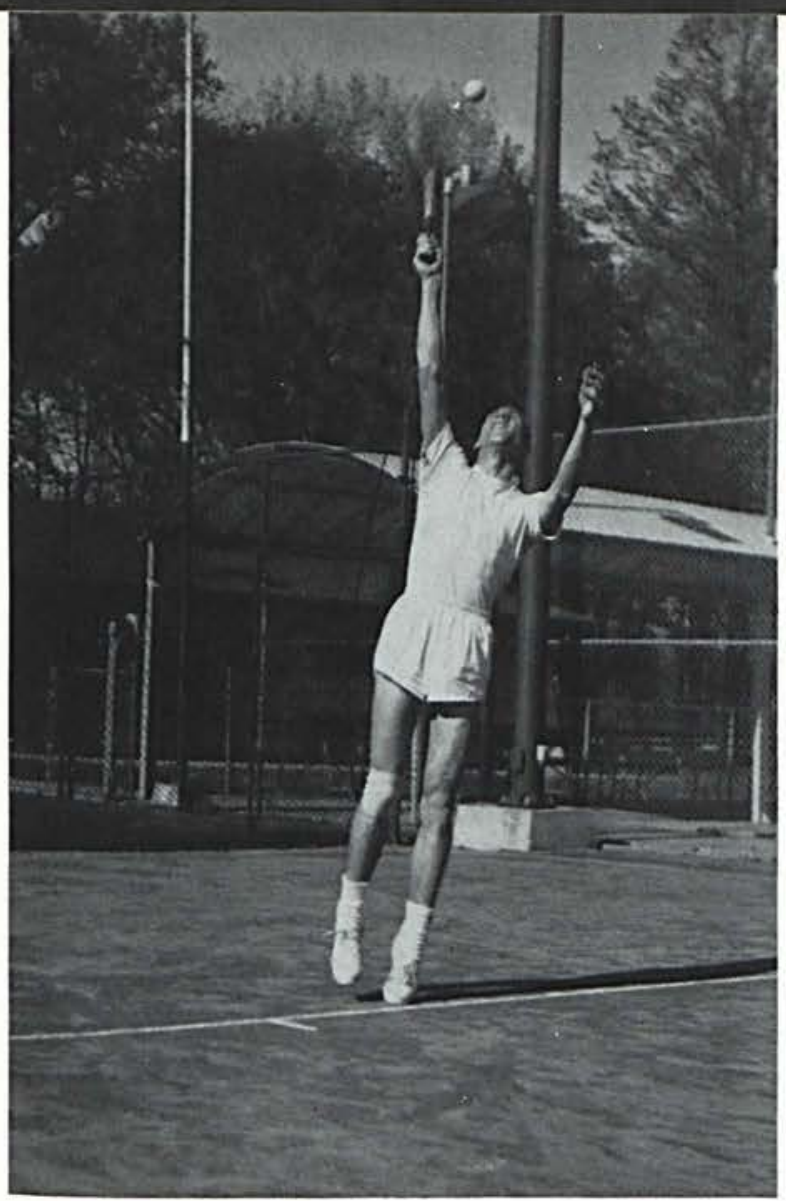

\section{Tennis}

Last spring the Yellowjacket netters found that the season was harder than anticipated. The squad won two out of their six games. In the Mid-Ohio League the Jackets tied for second place. Next year promises to be better with all this year's squad returning except Dick Wentzel and Land Moody who were lost by graduation.
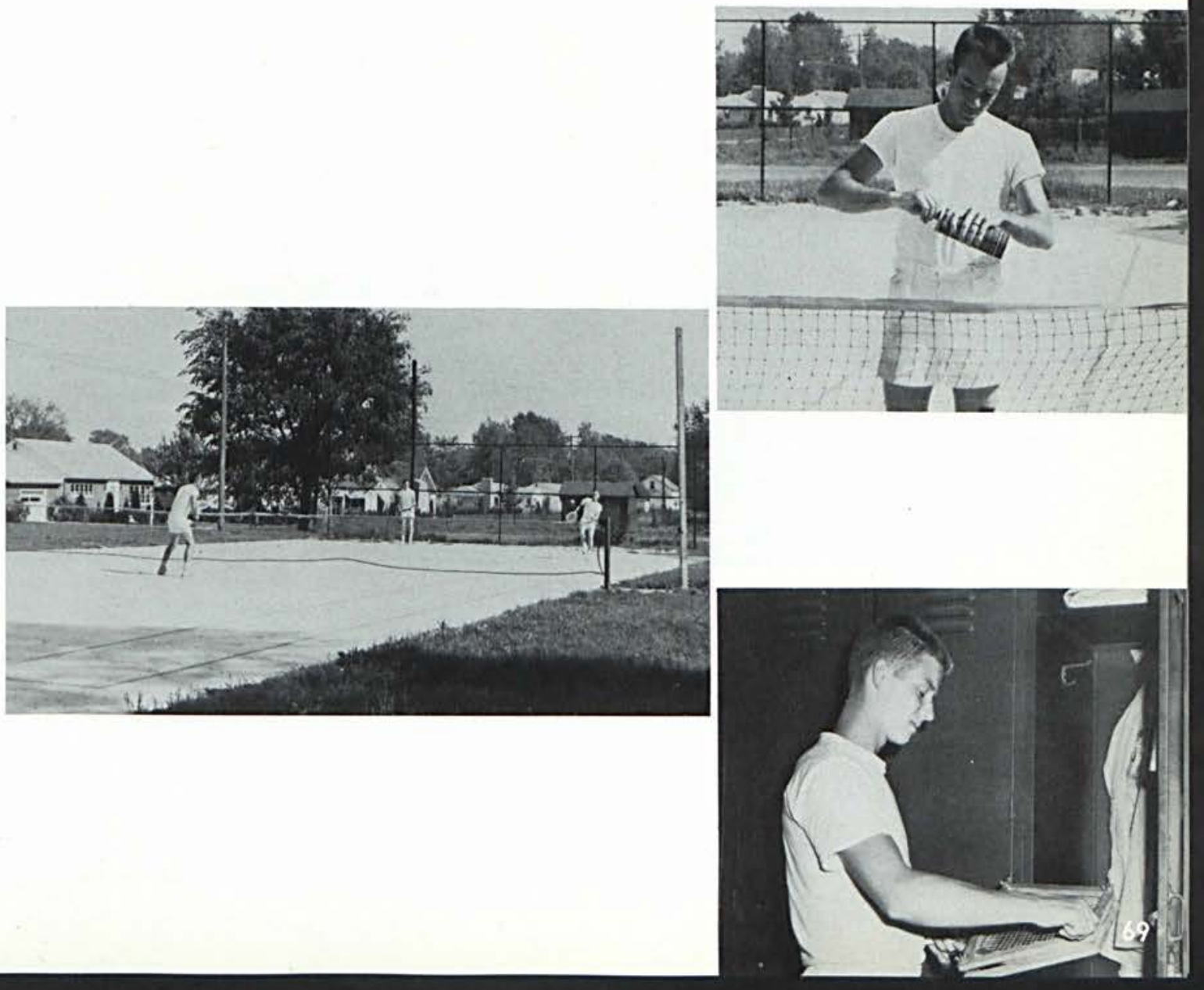


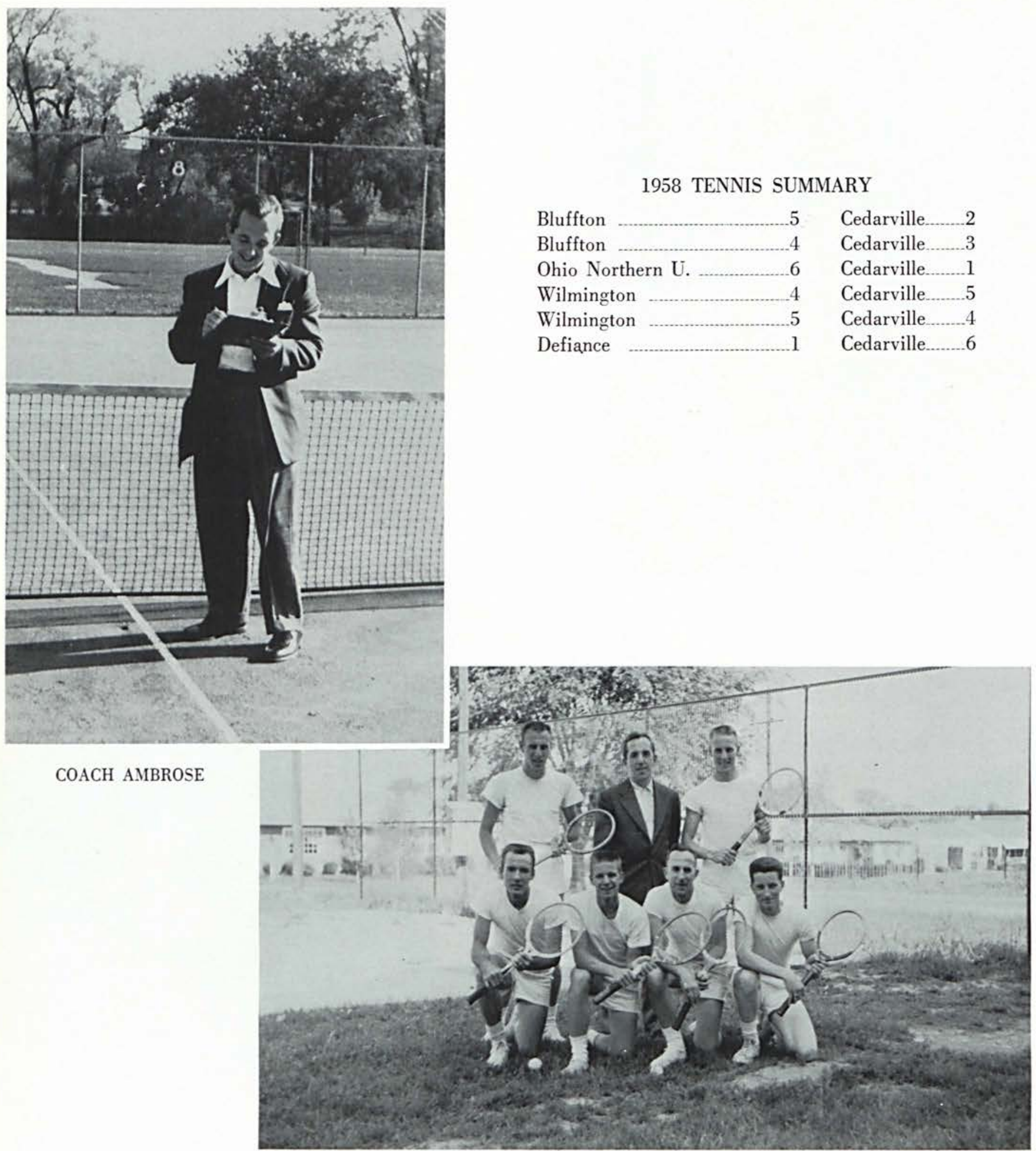

LEFT TO RIGHT: John Entner, Coach Ambrose, Jim Entner, Lane Moody, Keith Collett, Stuart Chaffe, Warren Woodard. 


\section{Prospective Students' Day}

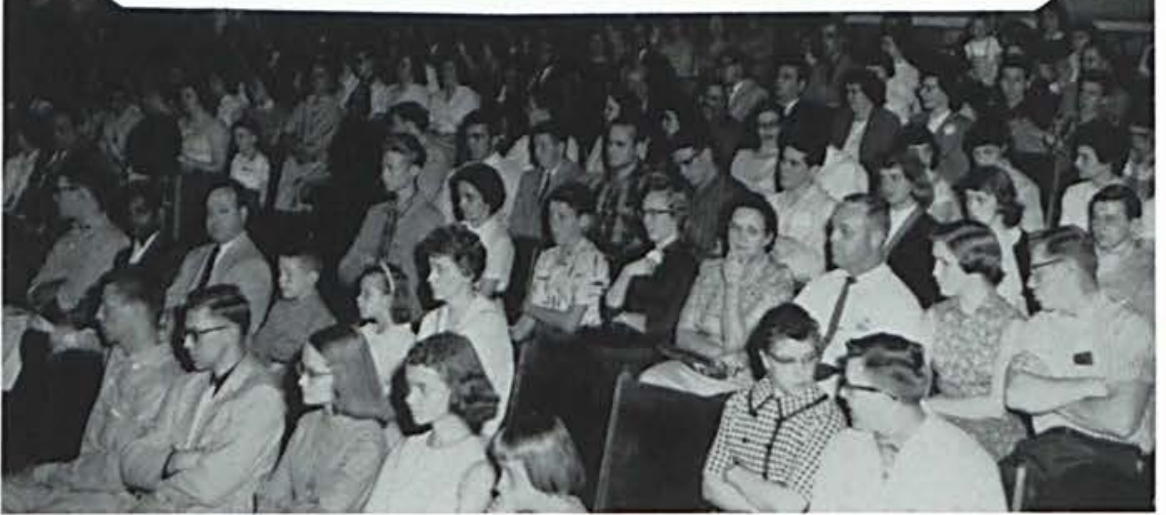

Students and guests enjoy film "Red River of Life."

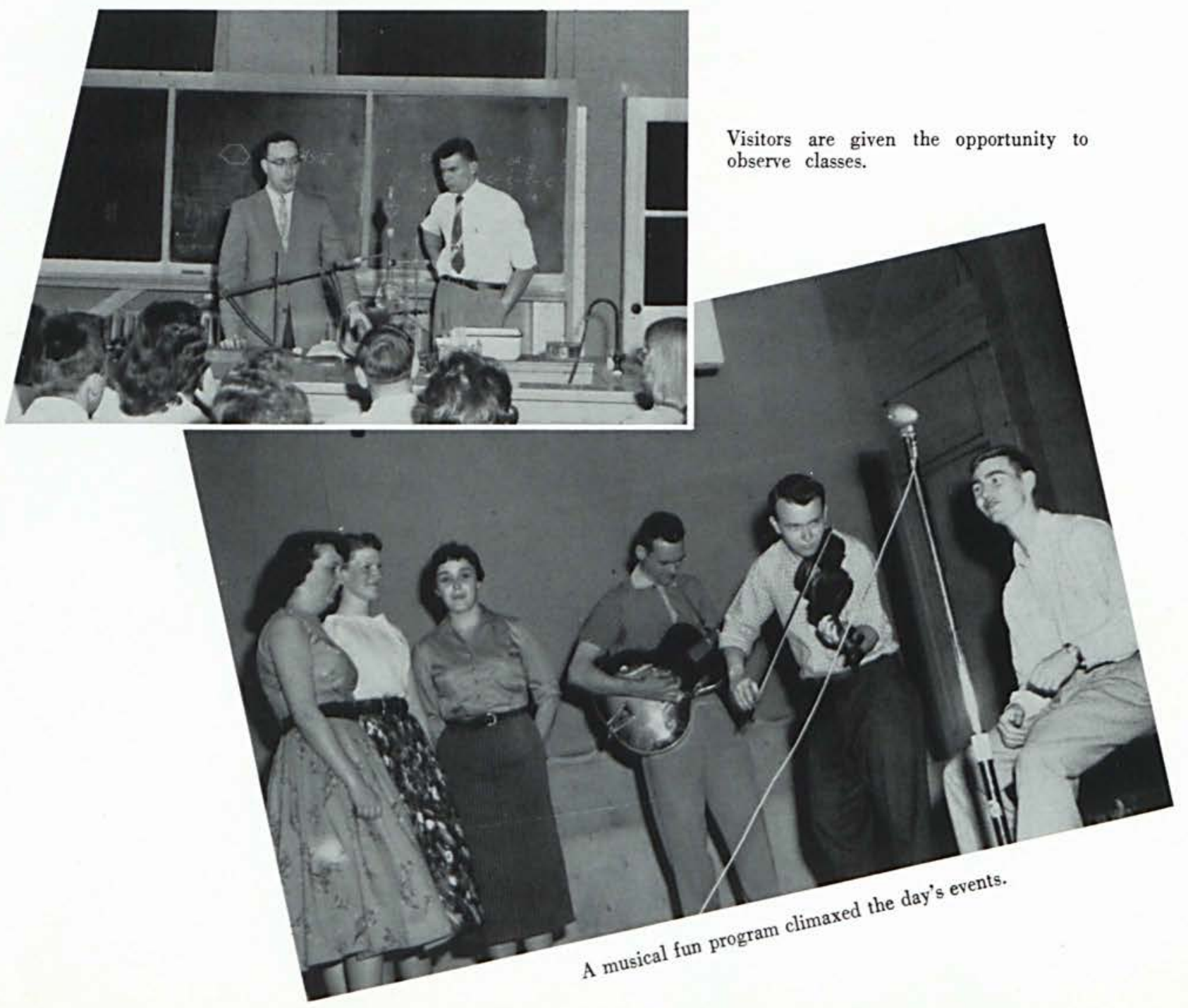





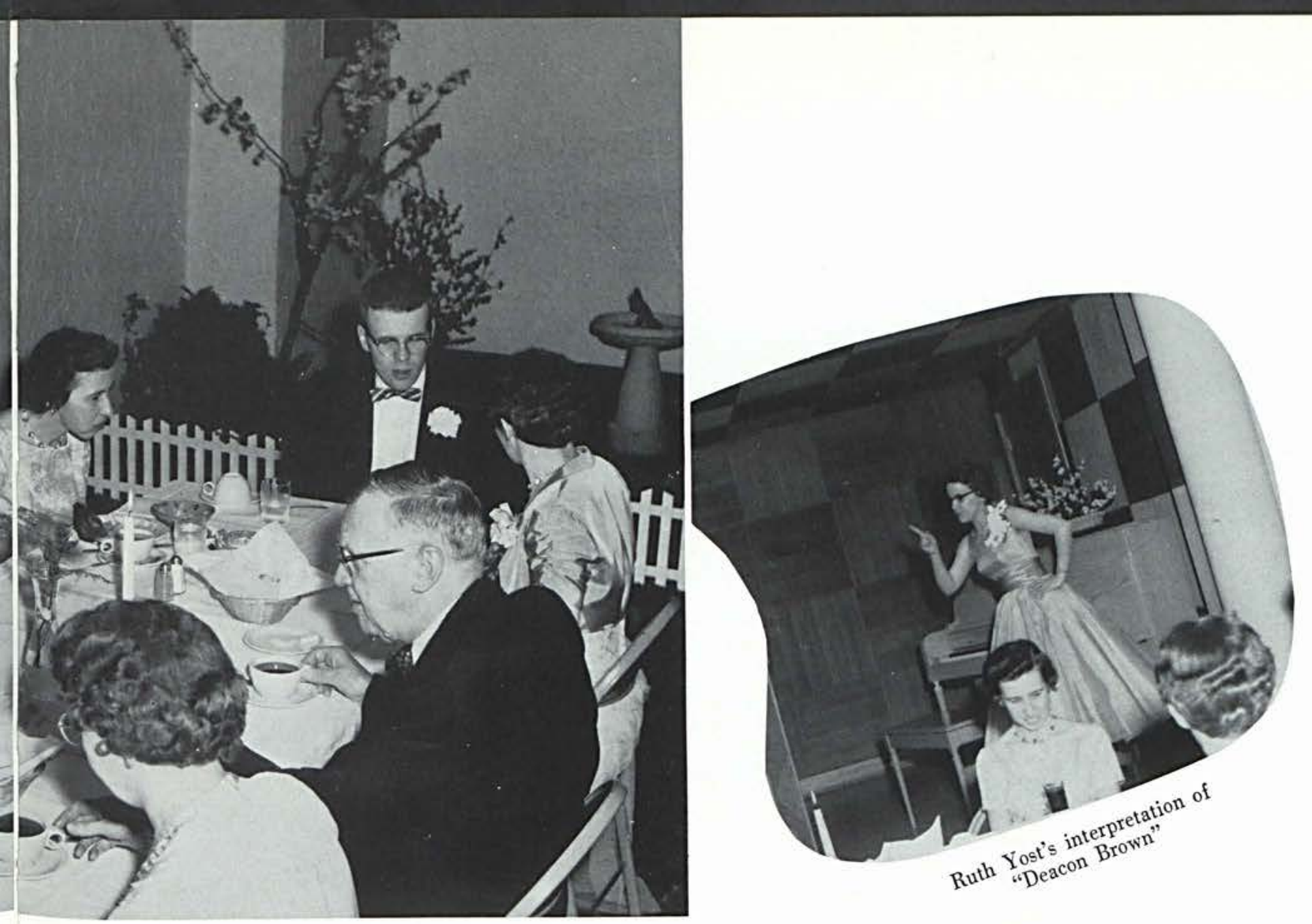

the red, white, and gold colors of the Senior class, added to the festivity of the event.

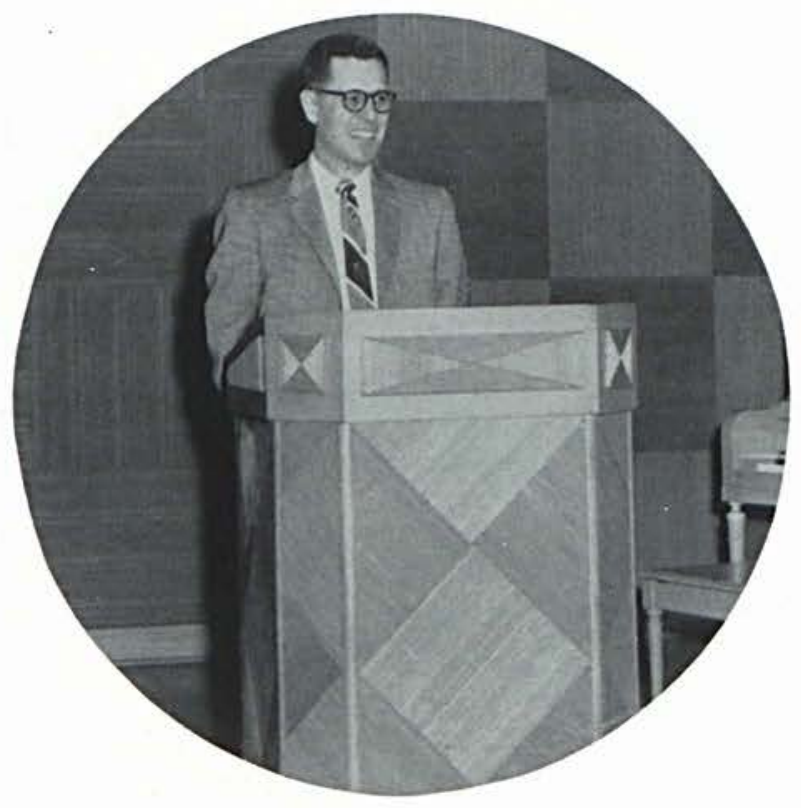

\section{Senior Banquet}

Dr. Kantzer, guest speaker,

"Theology in our Time" 


\section{Junior}

\section{Rock}

The Junior Class-some triumphant, some doubting

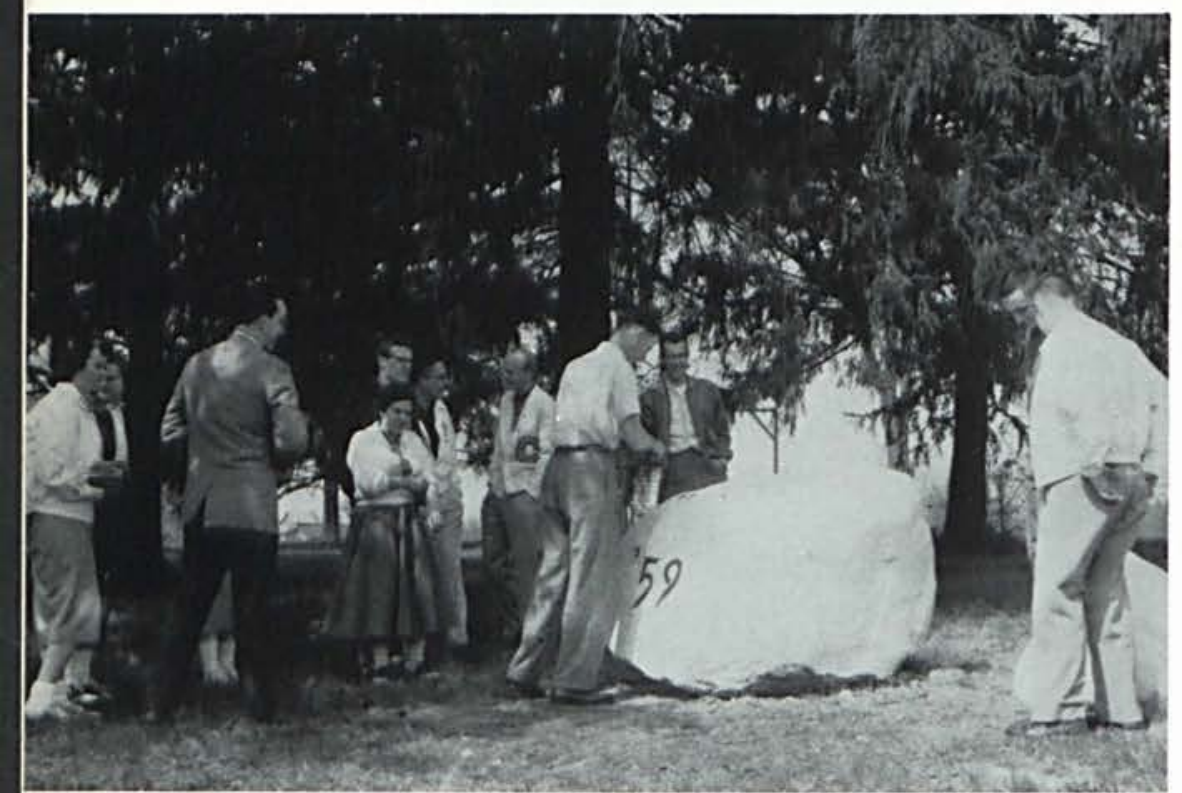

Cliff B., Junior class president, initials the rock.

The Conspirators

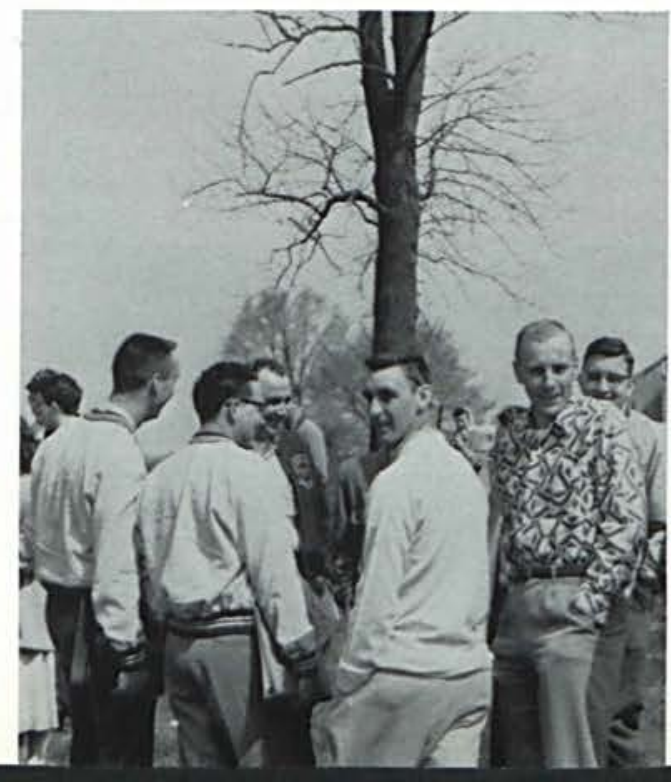




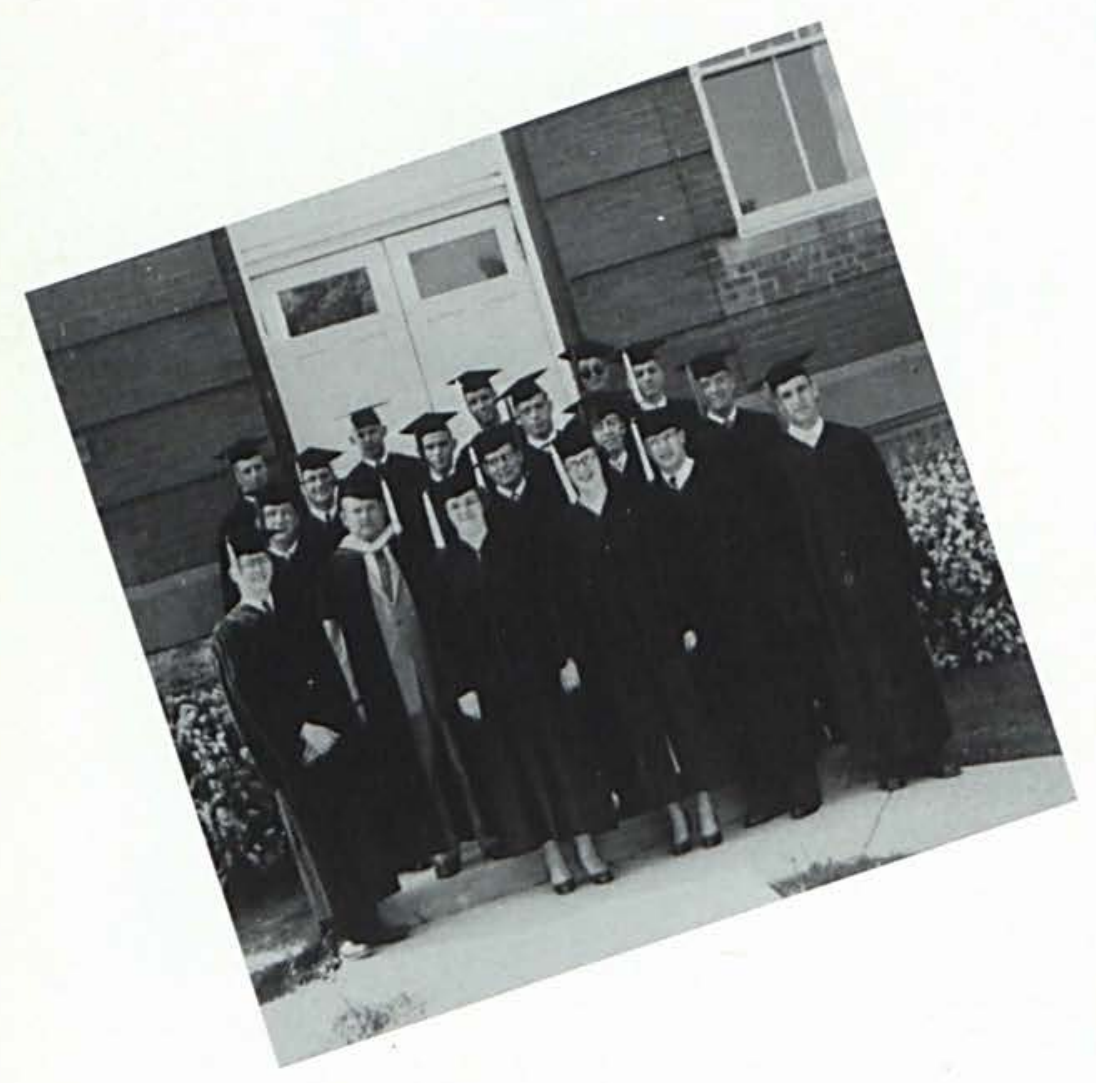

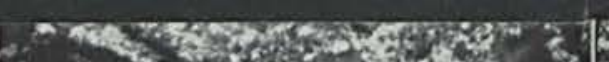

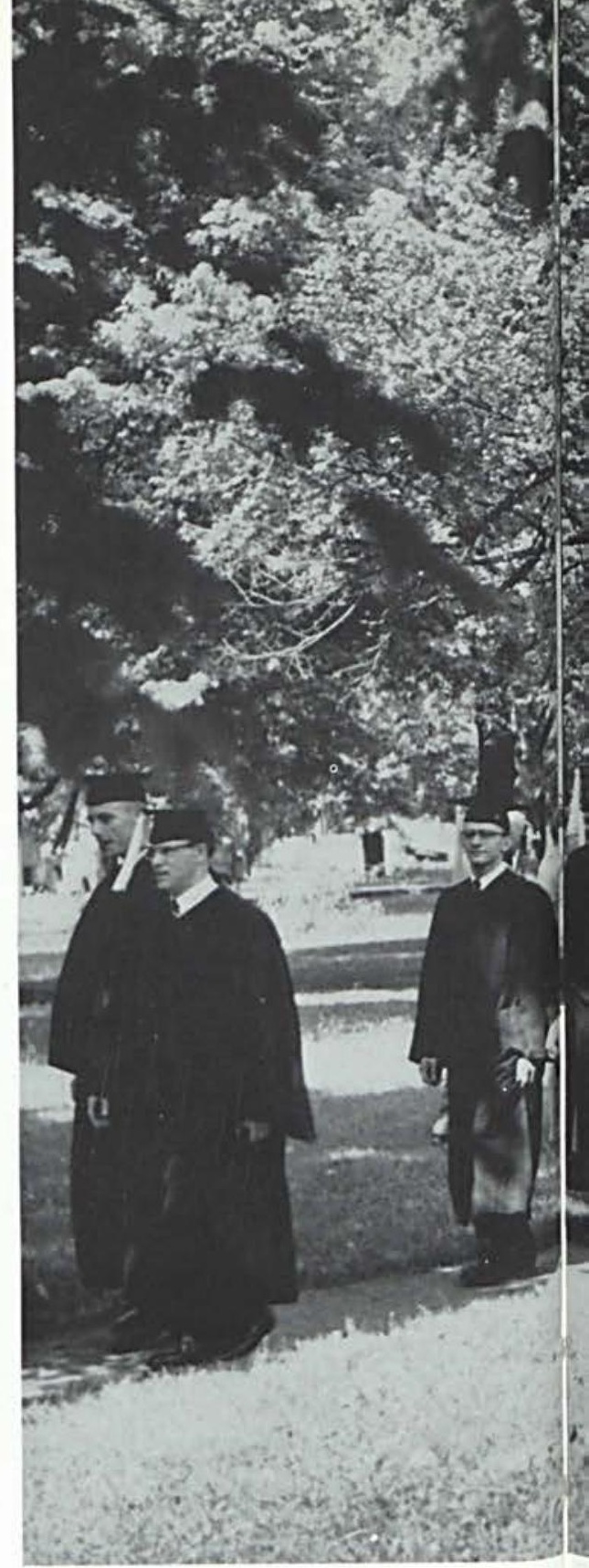




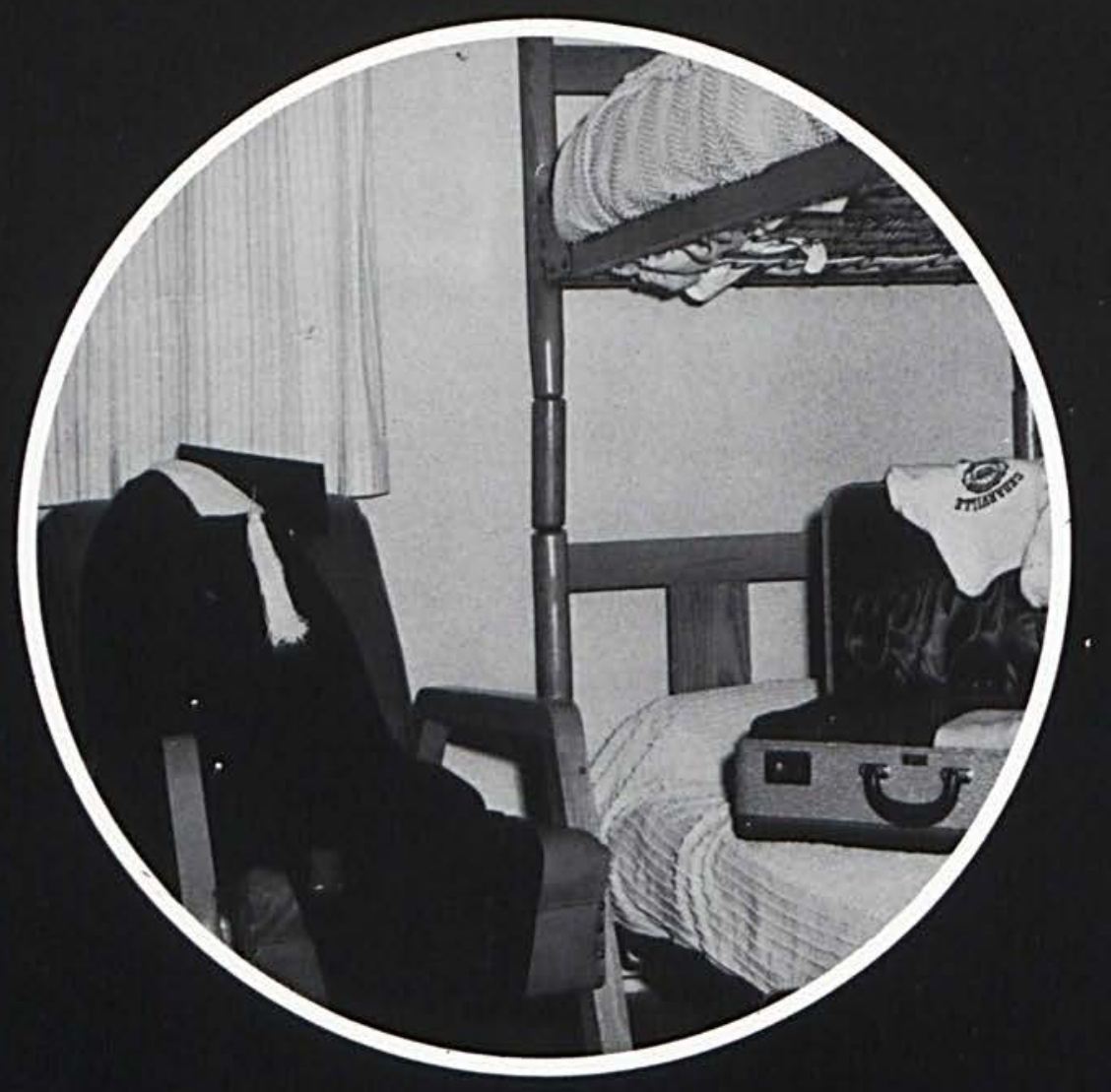

Yes, college days are a time of discovering ... but the most important discovery this year's graduating class made was that commencement marks the beginning of great discovery . . . "We cannot see beyond today as God can see ... many joys are waiting yet ..." 


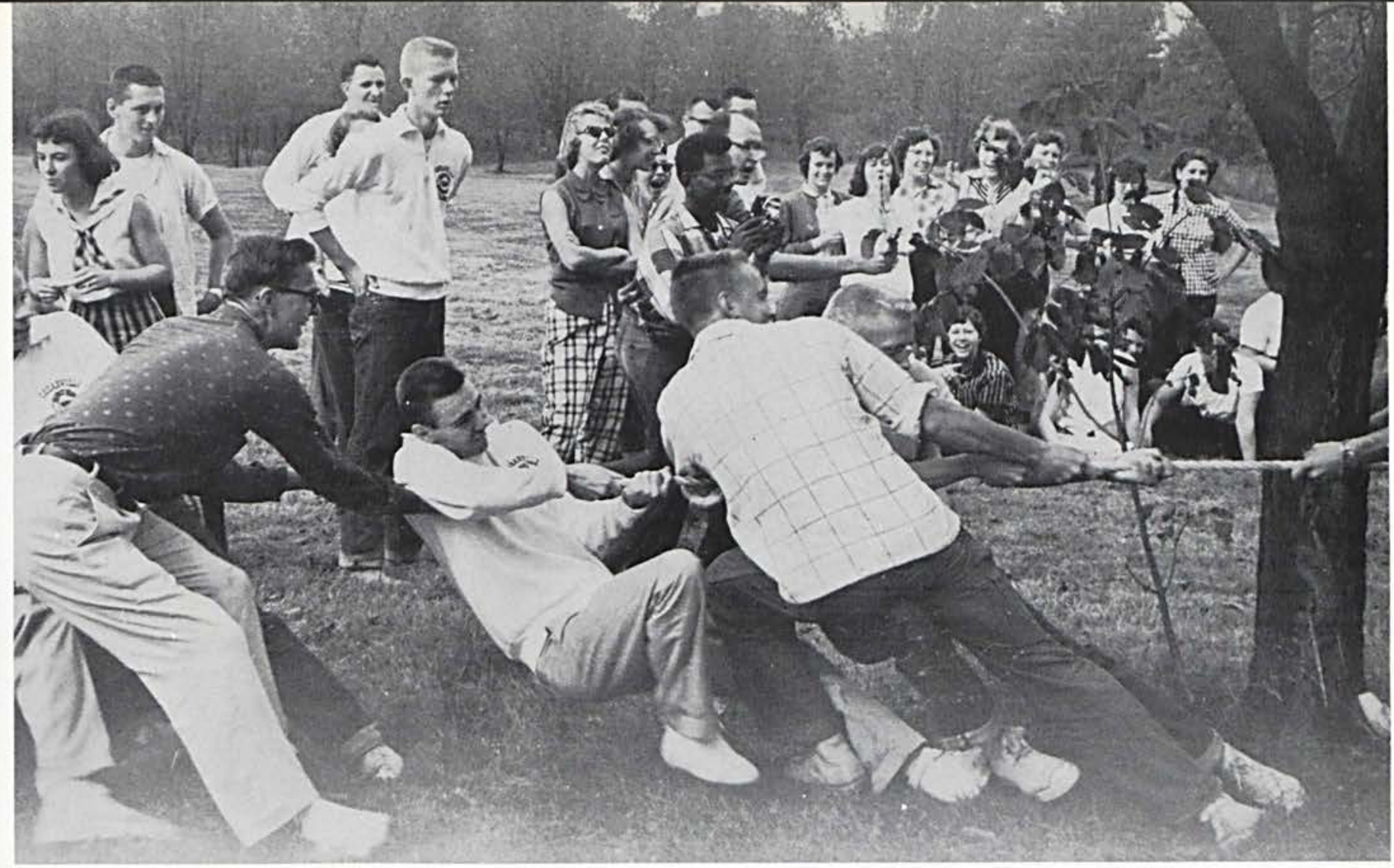

The beginning of a year-long battle

\section{Picnic}

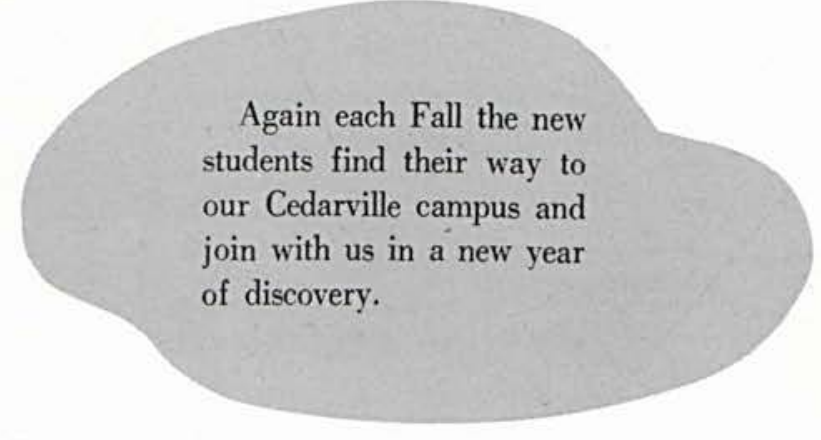

\section{Cleanup Day}
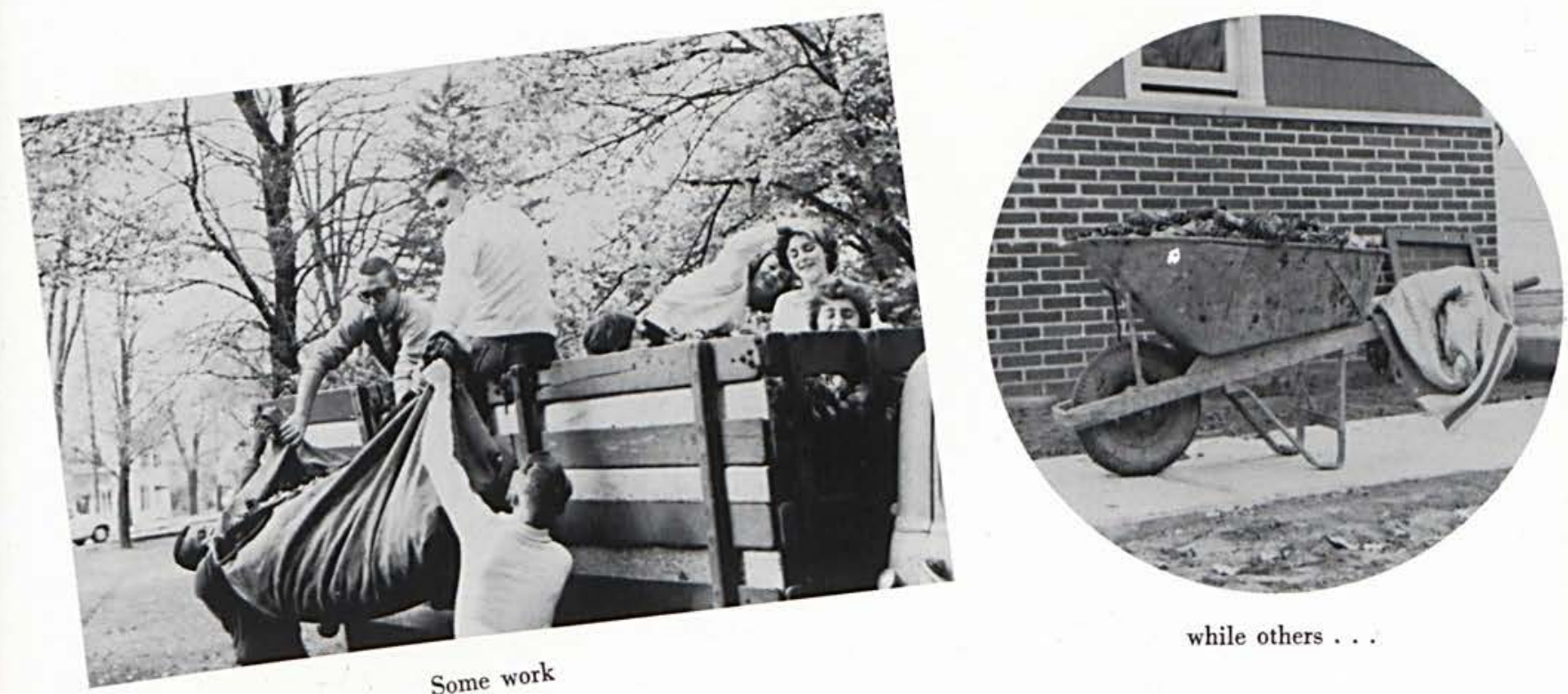


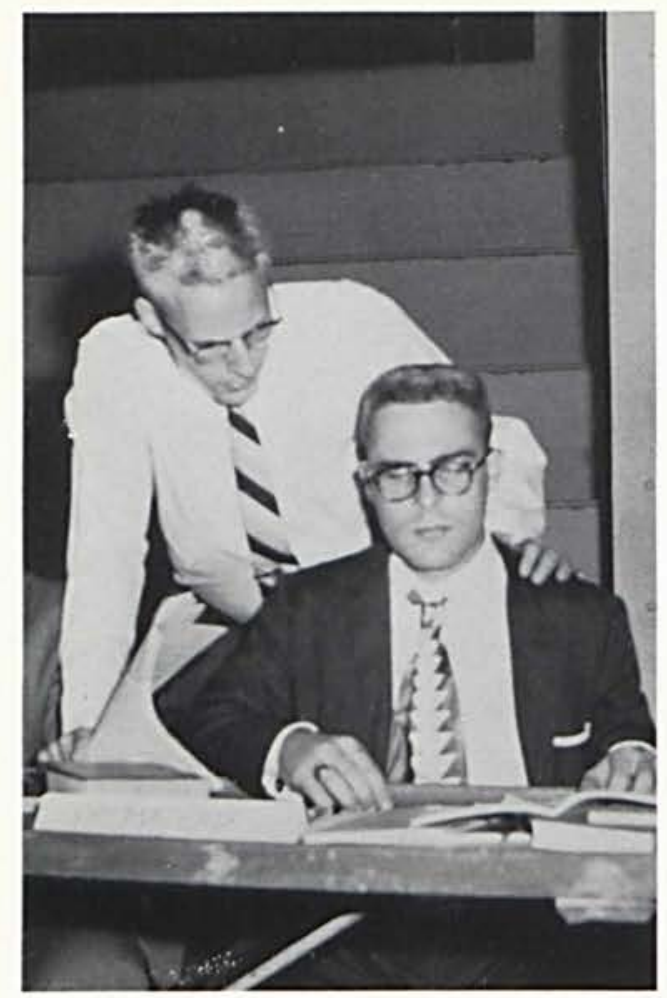

Student-Faculty Reception

What's up, Doc!
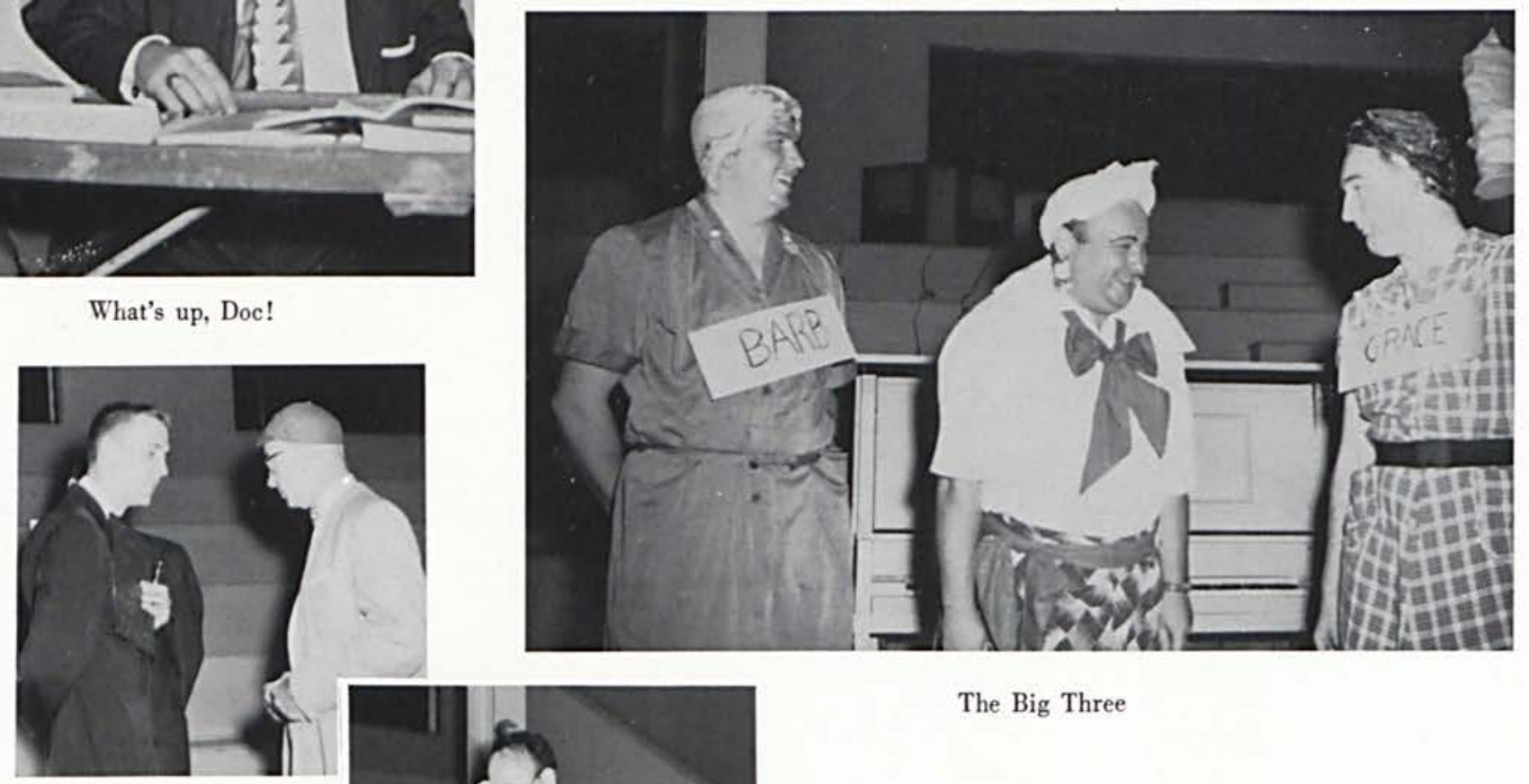

A Green Freshman

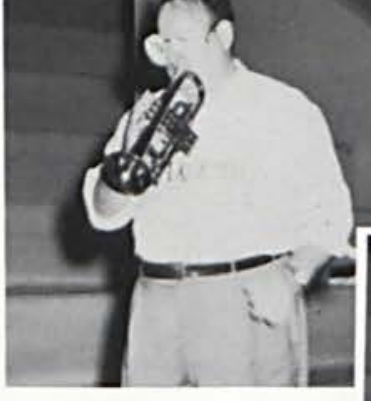

Mat Daveson

The Big Three

Beep-Beep 


\section{Homecoming Weekend}

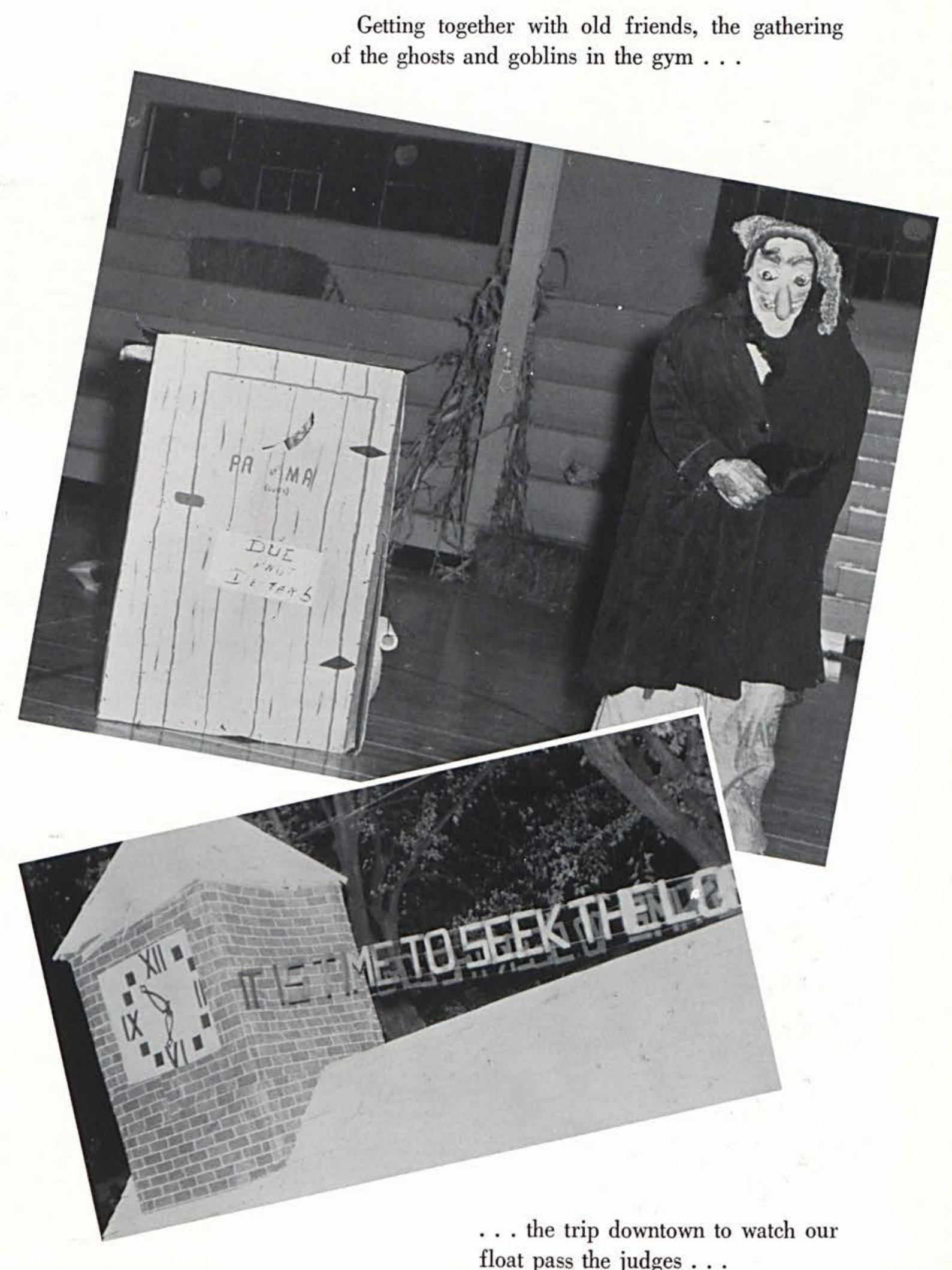




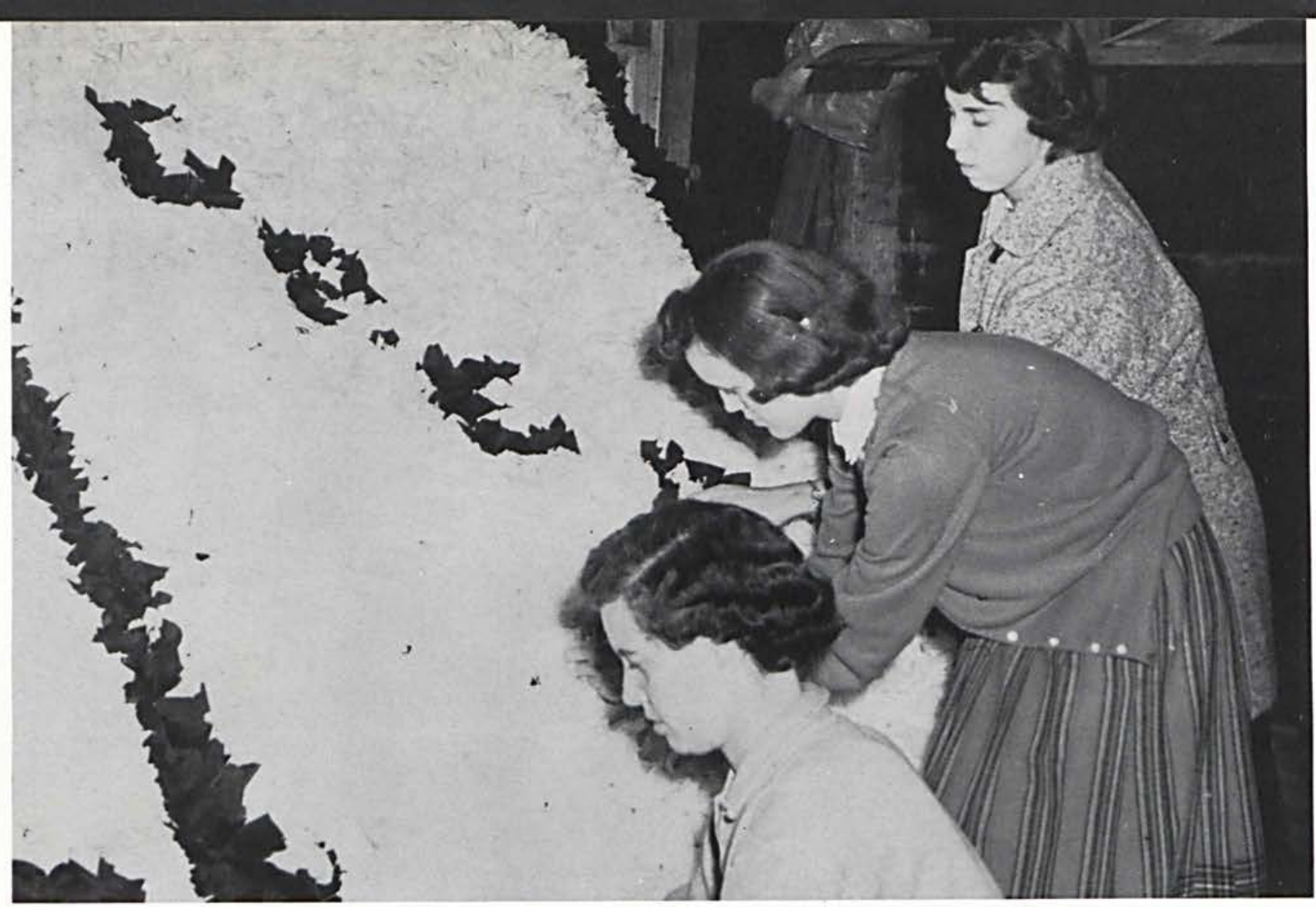

Many hours of work went into the building of the displays. The Freshman display won second prize.
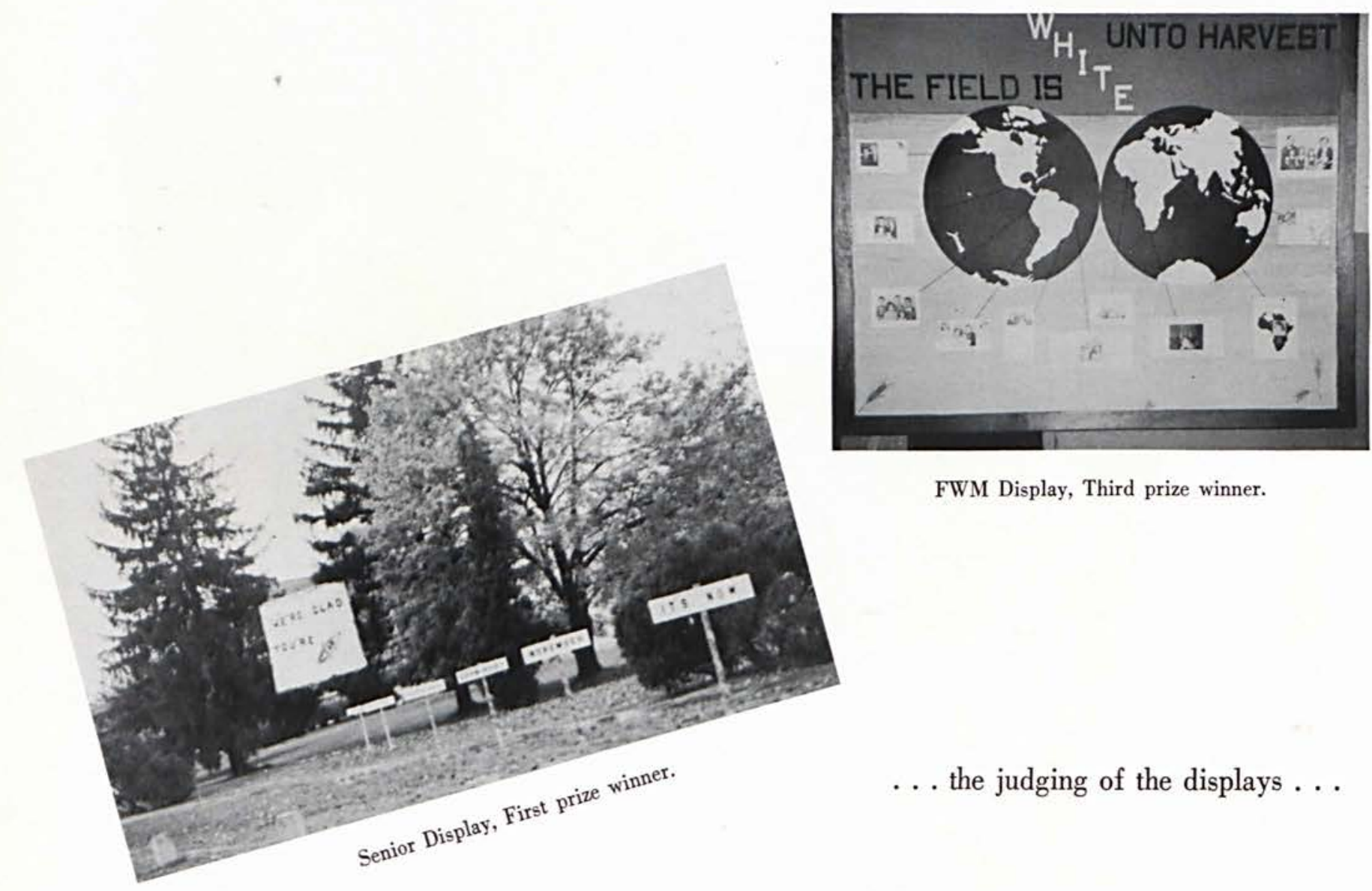

FWM Display, Third prize winner.

... the judging of the displays ... 


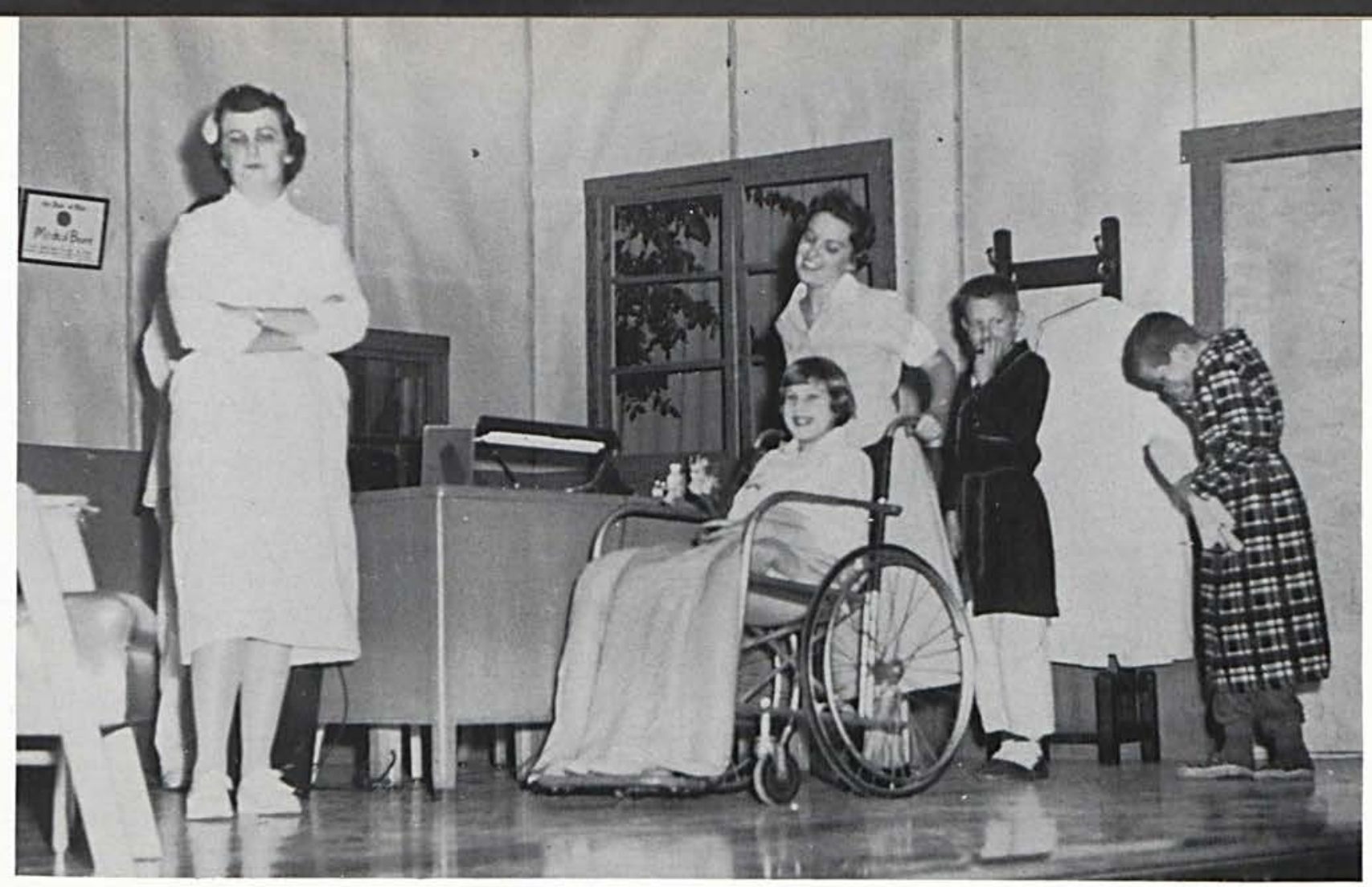

\section{Dr. Hudson's Secret Journal}

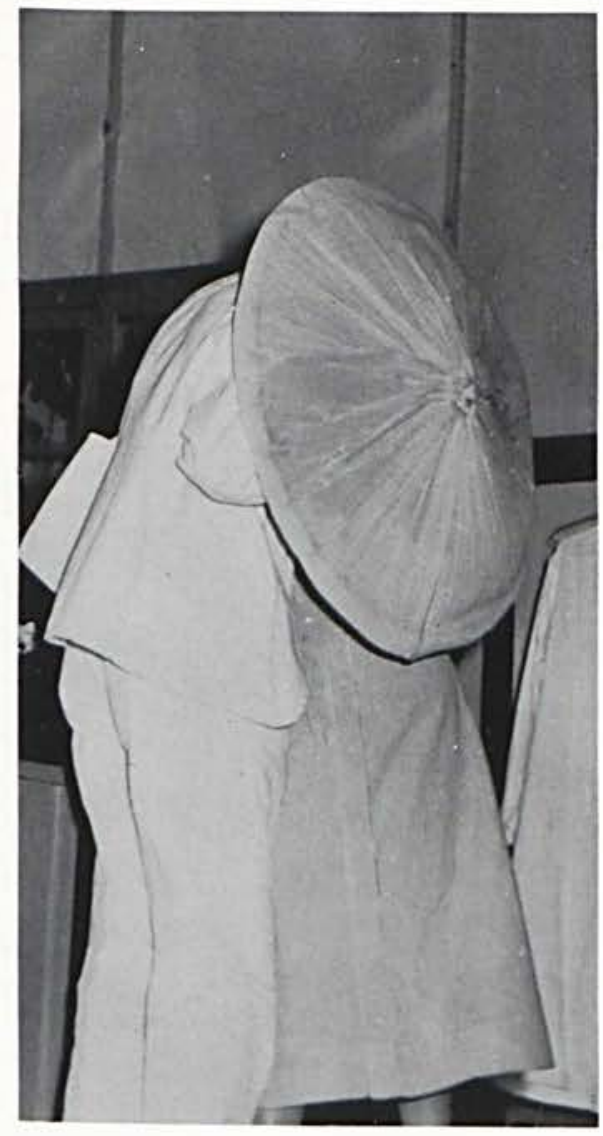

"What's your specialty, Barbara?"

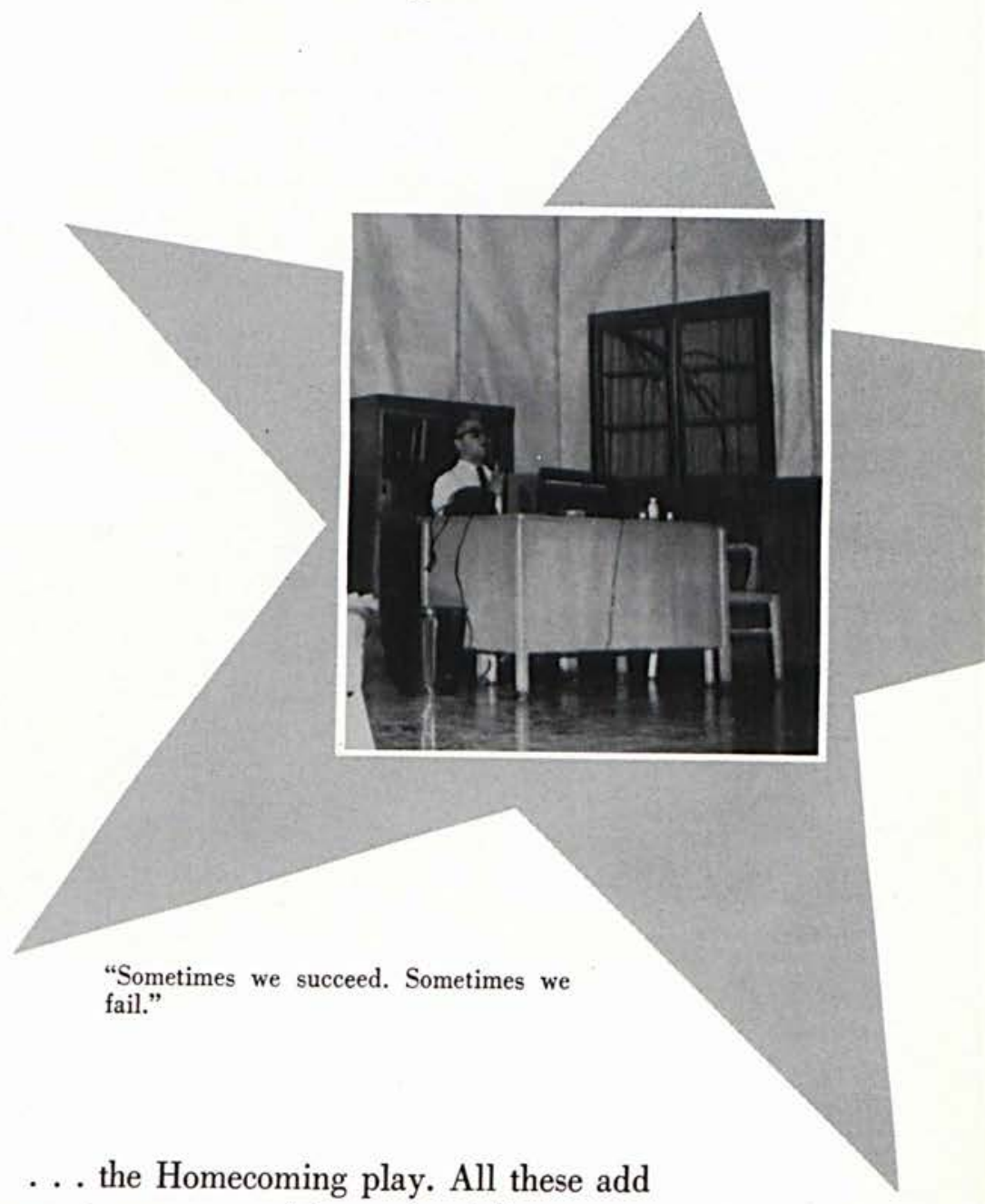

up to a memorable weekend." 


\section{Senior Sneak}

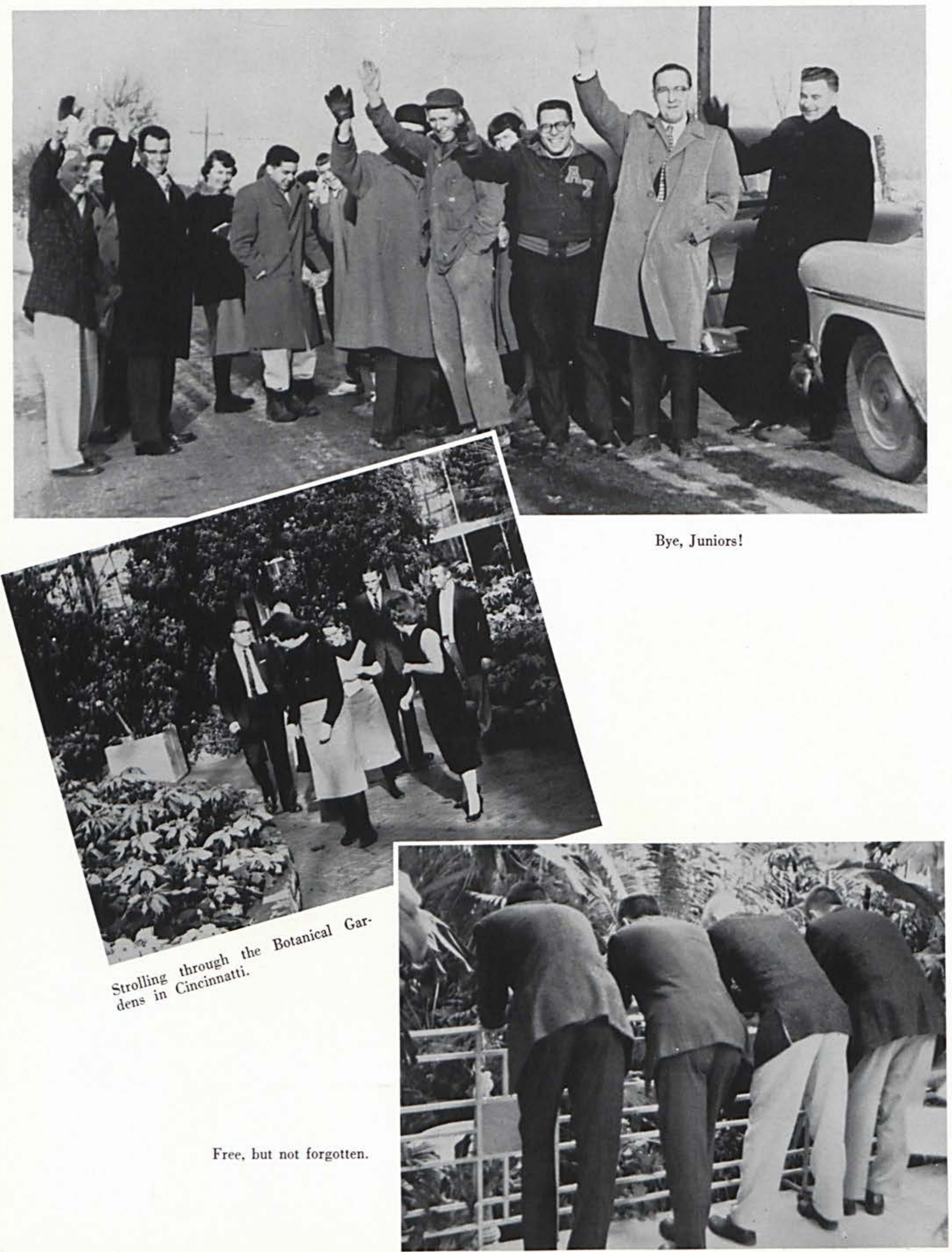




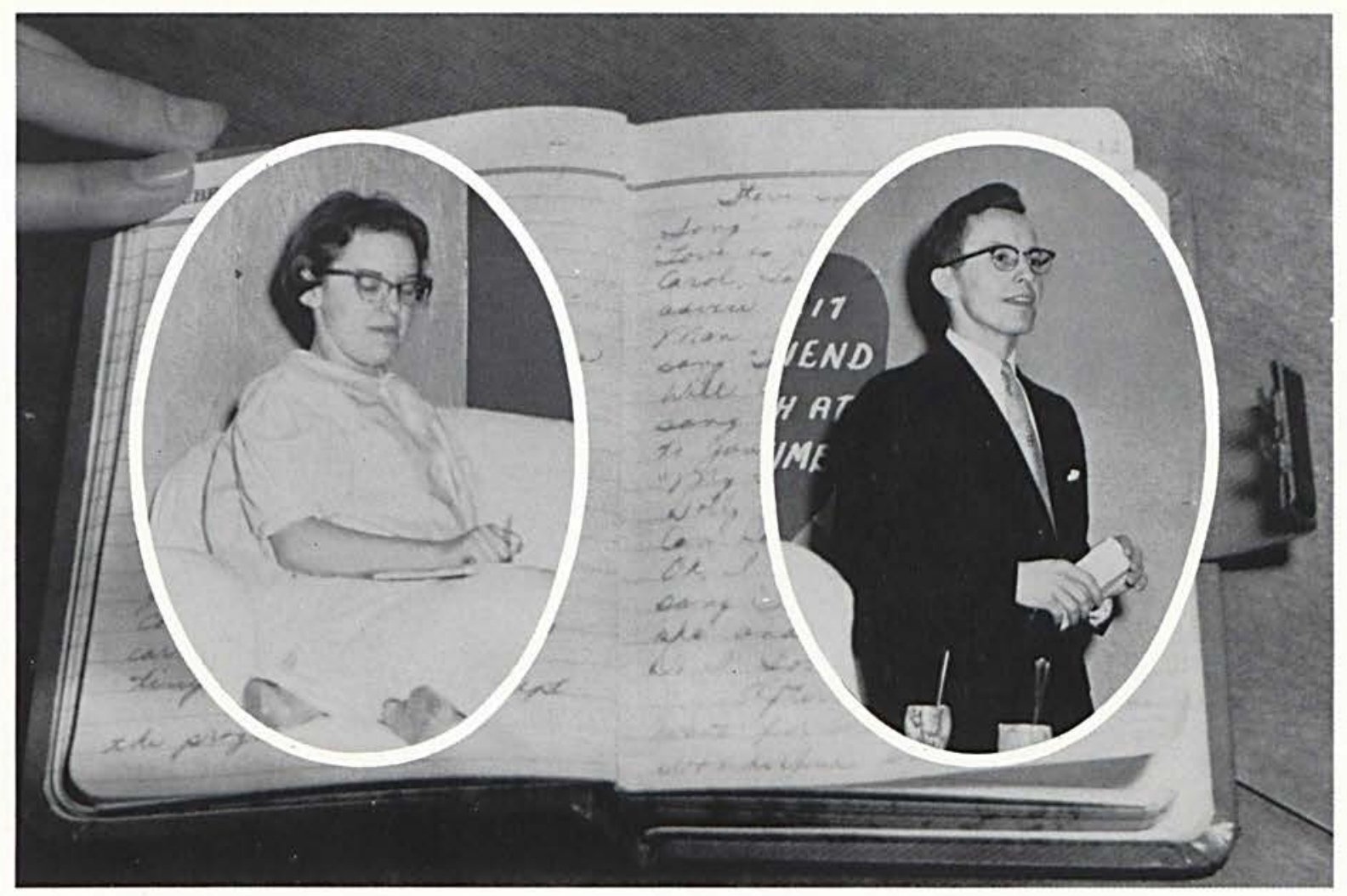

NARRATOR: Ruth Yost MASTER OF CEREMONIES: Rev. Glenn Greenwood

\section{Valentine Banquet}

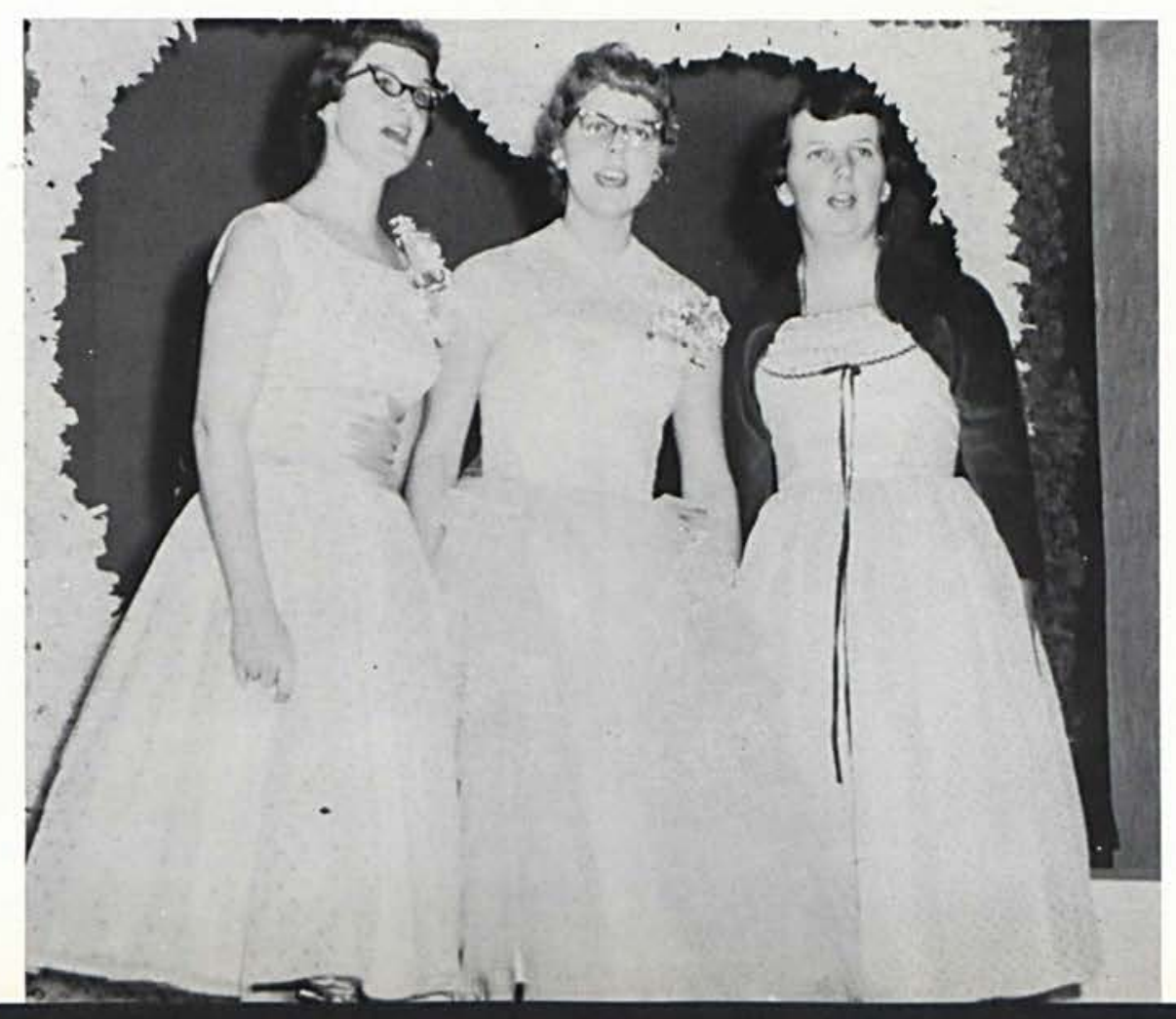




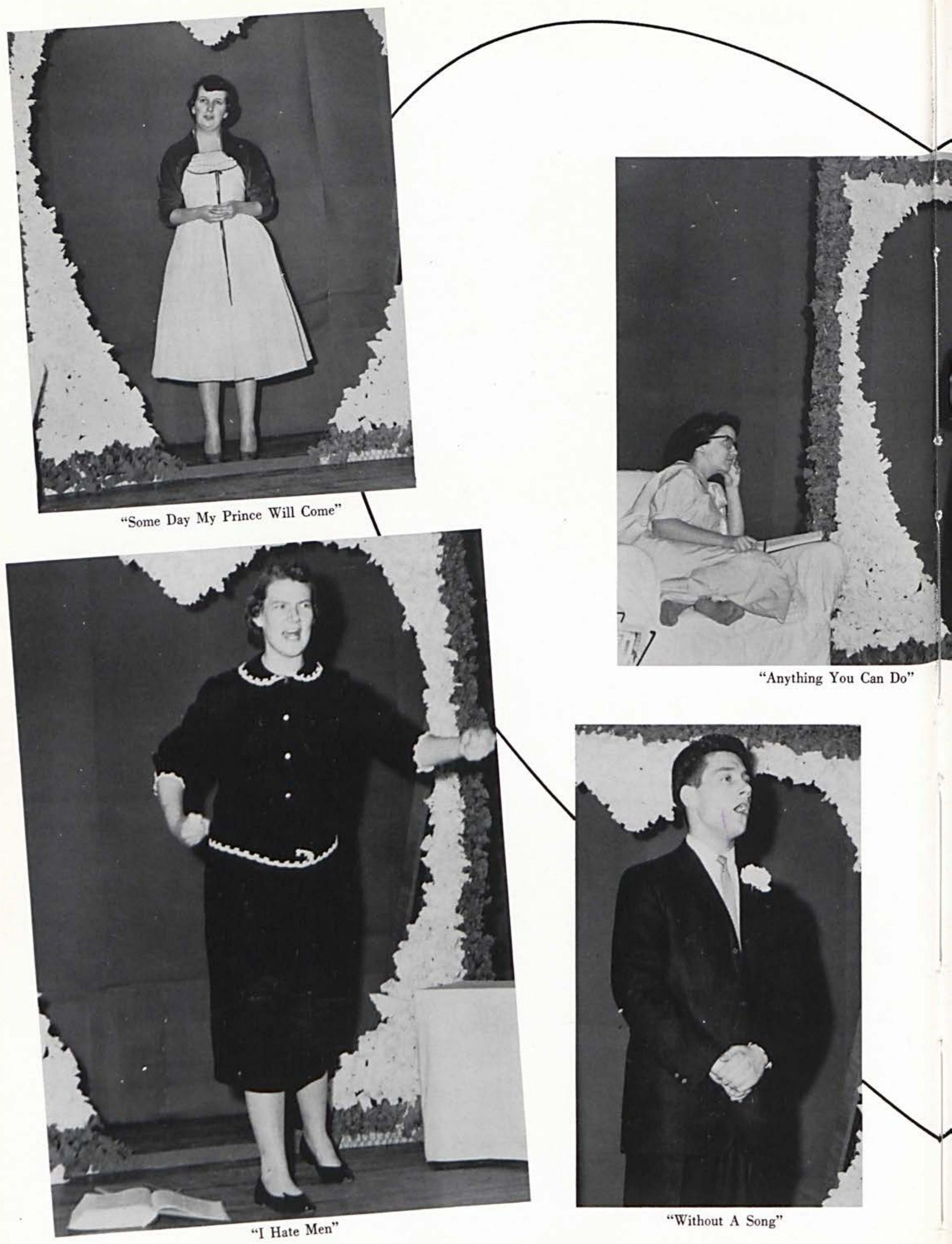




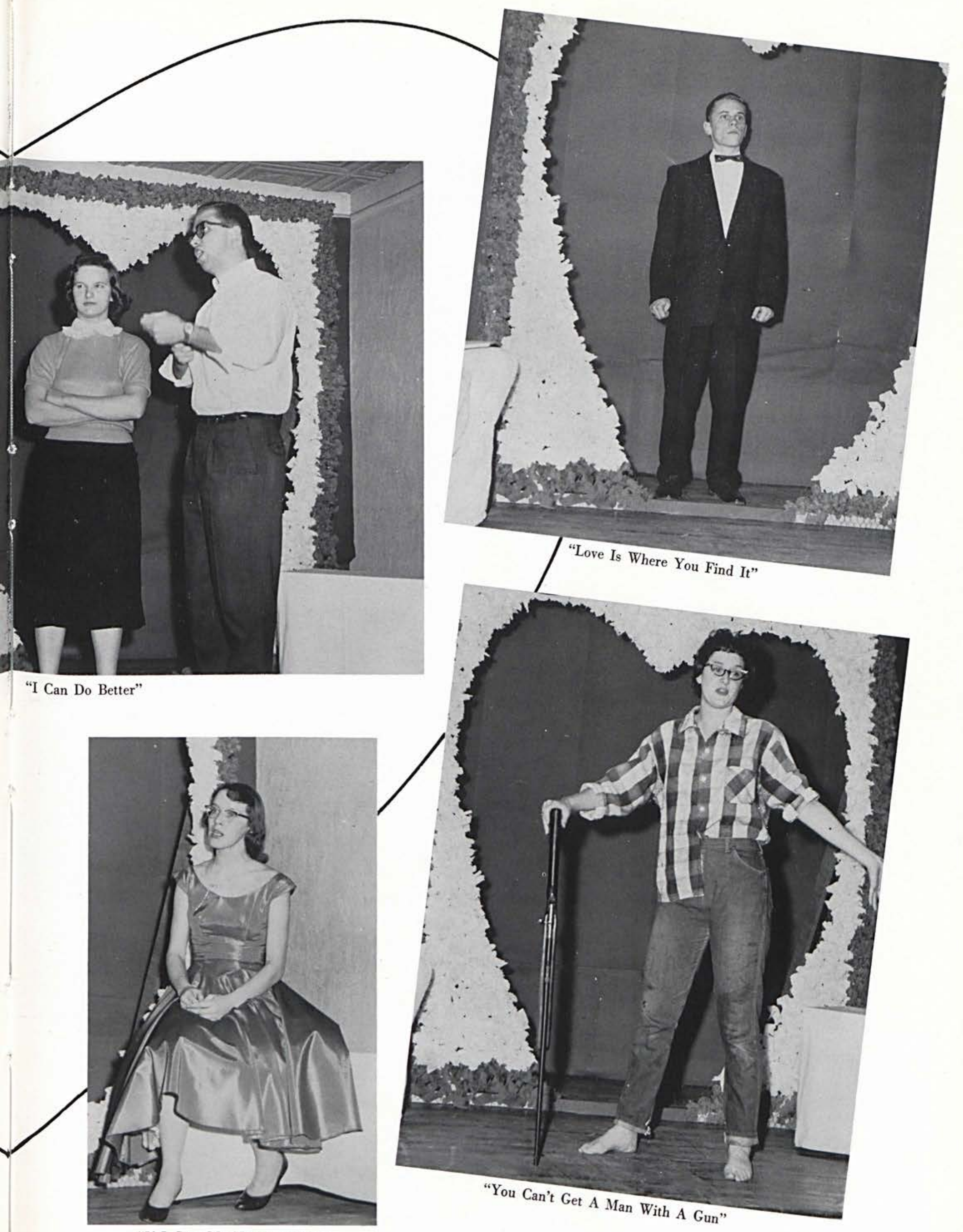

"If I Give My Heart To You" 


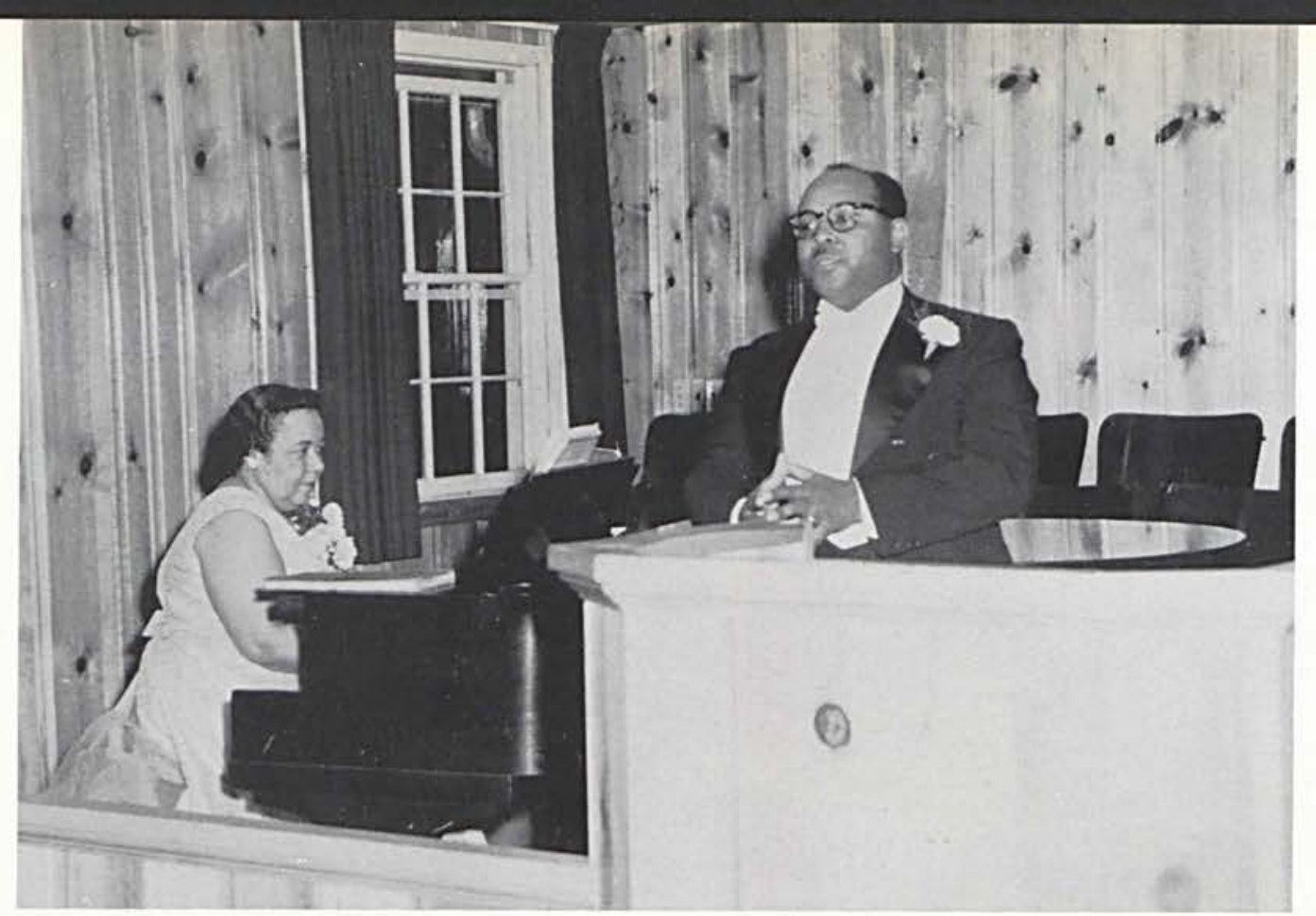

Oscar Henry, Tenor Soloist

\section{Artist Series}

\section{Oberlin String Quartet}

John Dalley, 2nd violin; Andor Toth, 1st violin; Peter Howard, violoncello; William Berman, viola.

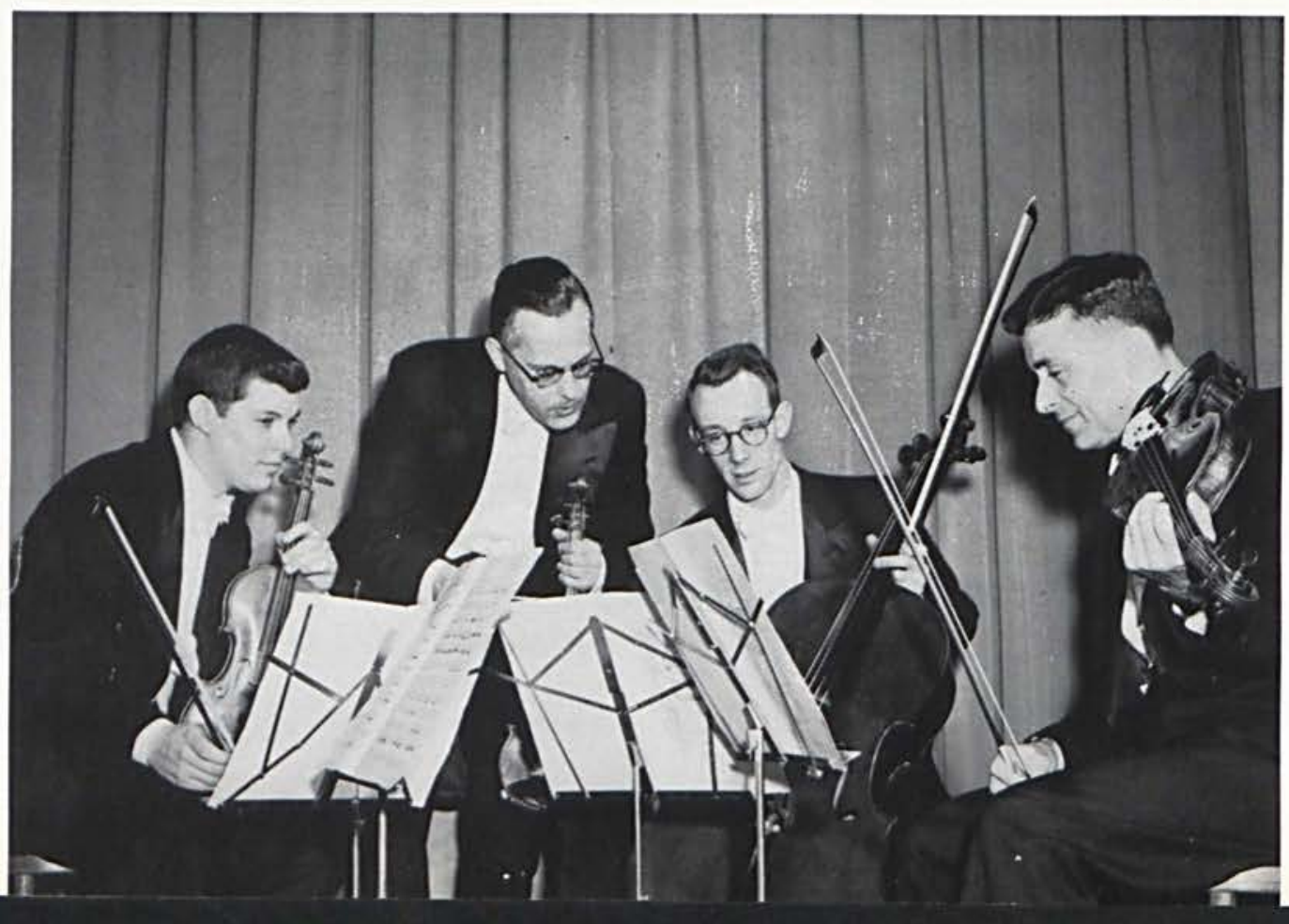




\section{Student Council}

The Student Council is chosen by the student body to act as their representatives to the faculty and administration. It strives to develop a well-rounded social, spiritual, and academic life on the campus.

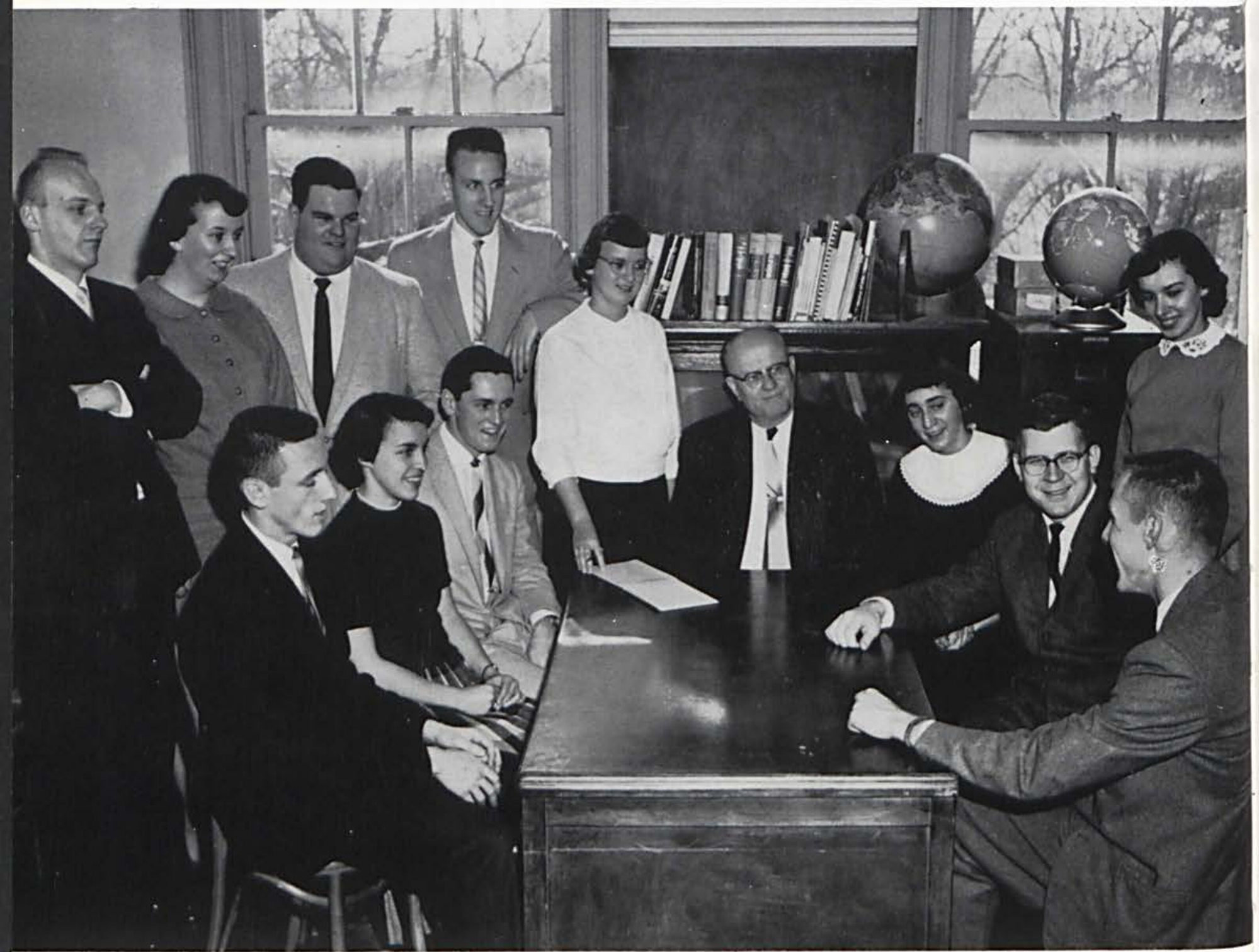

SEATED: Robert Howder, Esther Weiss, Merlin Ager, Dr. Maddox, advisor; Kay Lamb, Robert Humphreys, John Entner. STANDING: David Thomas, Grace Willetts, Tony Kilbourn, James Entner, Carol Johnson, Lucy Lyons.

President JOHN ENTNER

Vice President
MERLIN AGER
Secretary CAROL JOHNSON

Treasurer ROBERT HOWDER 


\section{Fellowship}

\section{for \\ World Missions}

The purpose of Fellowship for World Missions is to remember our missionaries in prayer, to create an interest within the student body for missions, and to correspond with missionaries with whom we are familiar.

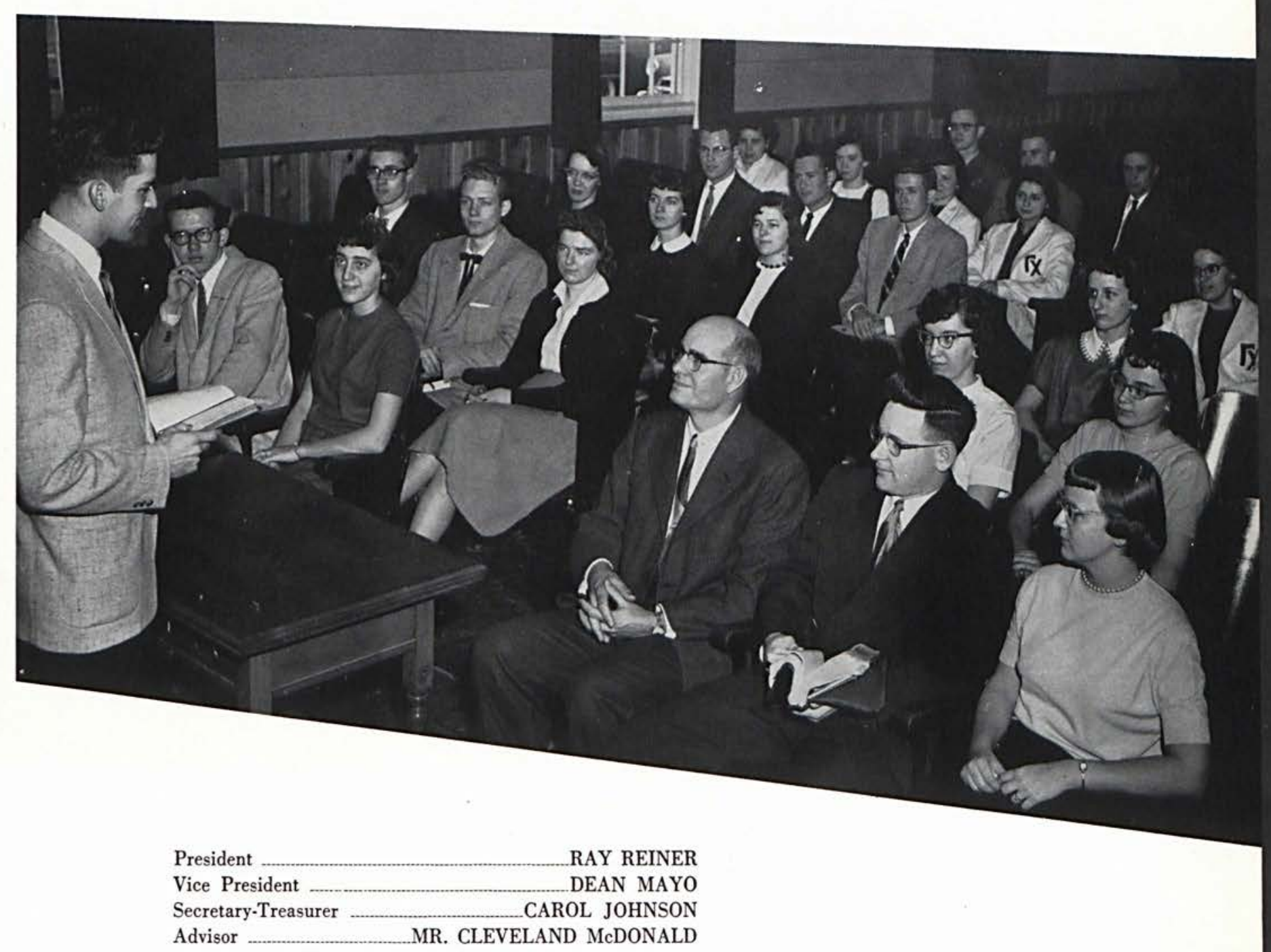


The Varsity "C" Club encourages and promotes intramural and intercollegiate athletics in order to bring glory to our Lord and Saviour Jesus Christ and to produce good sportsmanship and strong bodies.

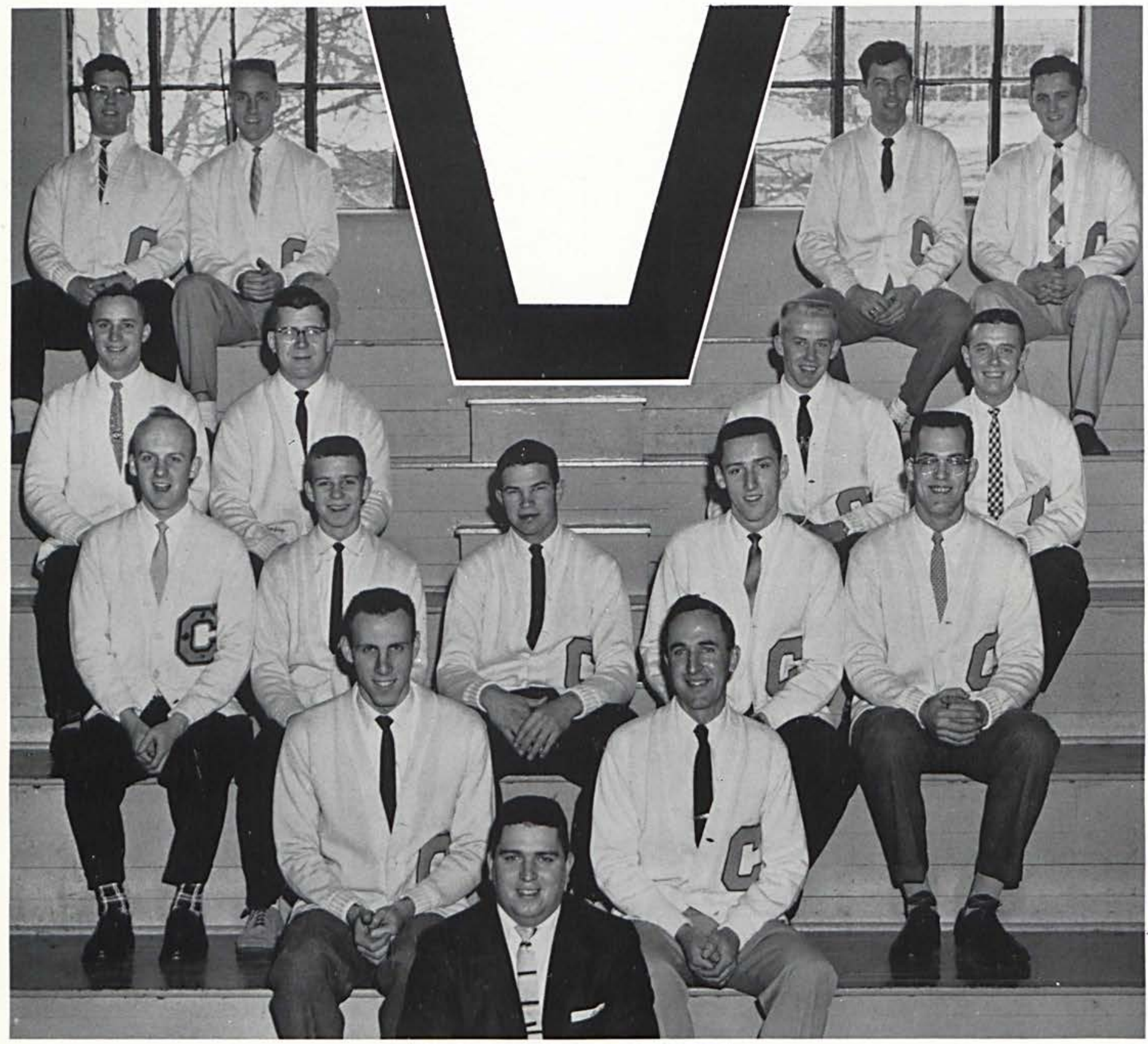

Dave Dautel, Jim Entner, Dave Cochran, Bob Humphreys, Dave Thomas, Keith Collett, Pete Reese, John Entner.

President

Vice President OHN ENTNER Chaplain STU CHAFFE BOB HUMPHREYS
Willy Wentzel, Merlin Ager, Dan Purdy, Les Webster, Bob Howder, Roy Carr, Stu Chaffe, Coach Bowser.

Secretary DAVE THOMAS Treasurer Business Manager KEITH COLLETT BOB HOWDER 


\section{Dramatics Club}

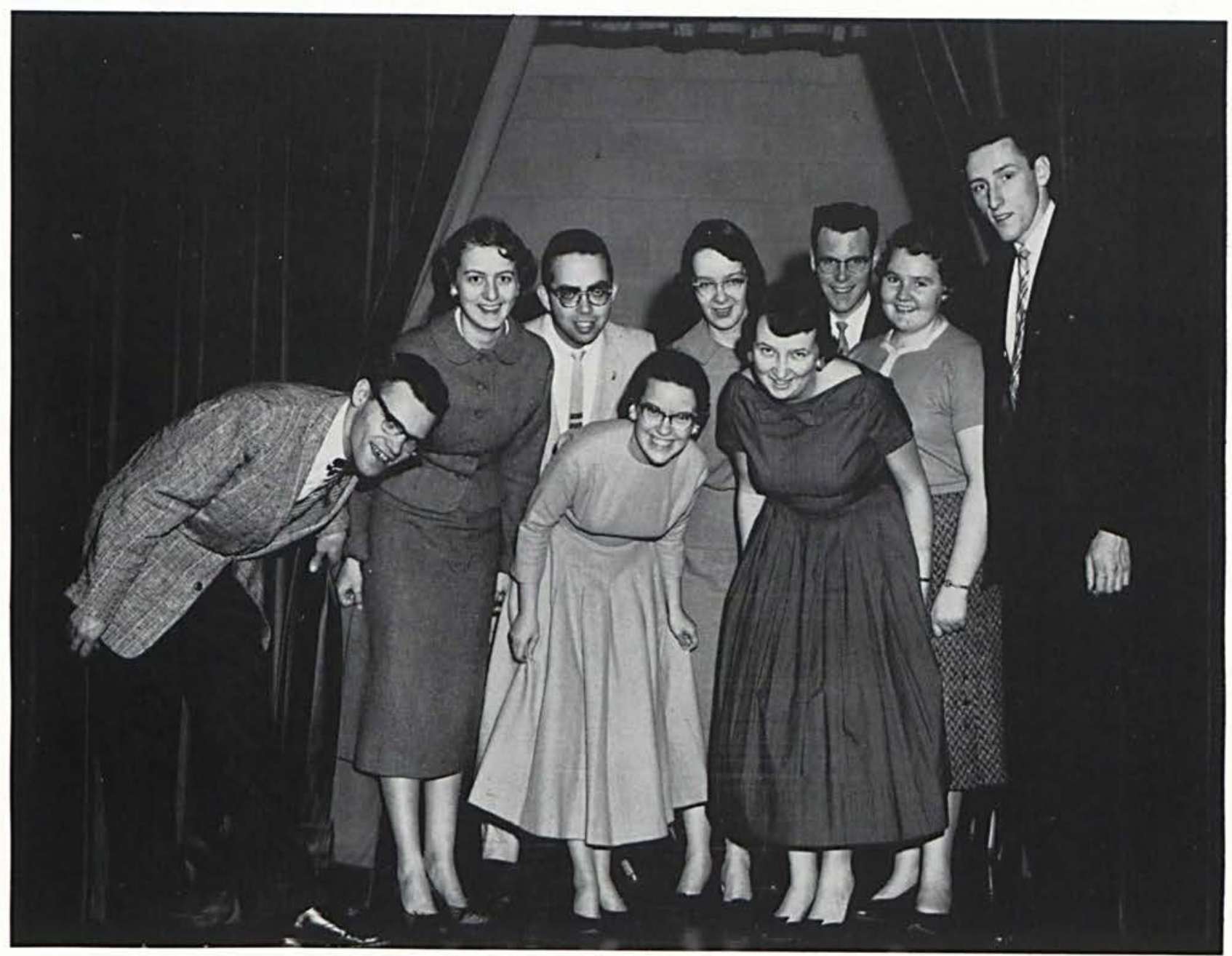

From L. to R.: Keith Webster, Lois Jeremiah, Dave Matson, Ruth Yost, Rosemary Smith, Grace Willetts, Dwayne Frank, Delores Osborn, Bob Howder.

"The purpose of the Dramatics Club is to provide the opportunity for dramatic experience through occasional productions for campus events and to recognize students who have contributed this service."

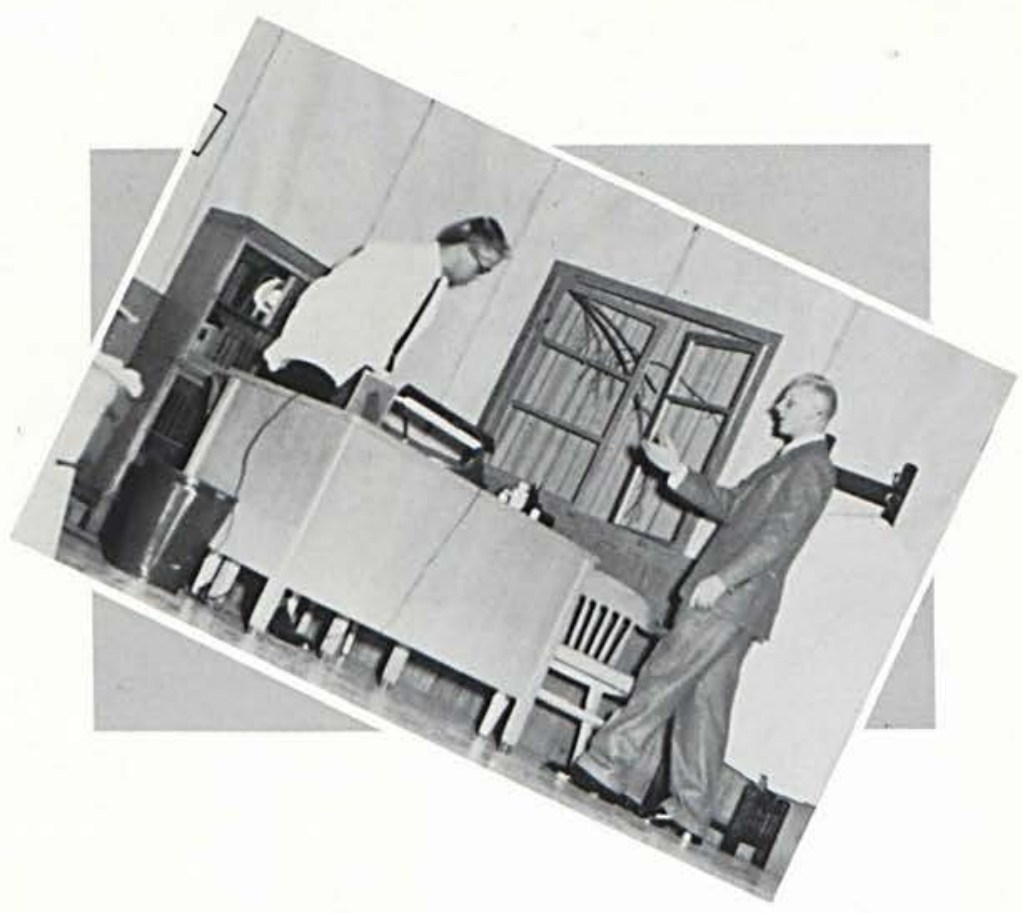



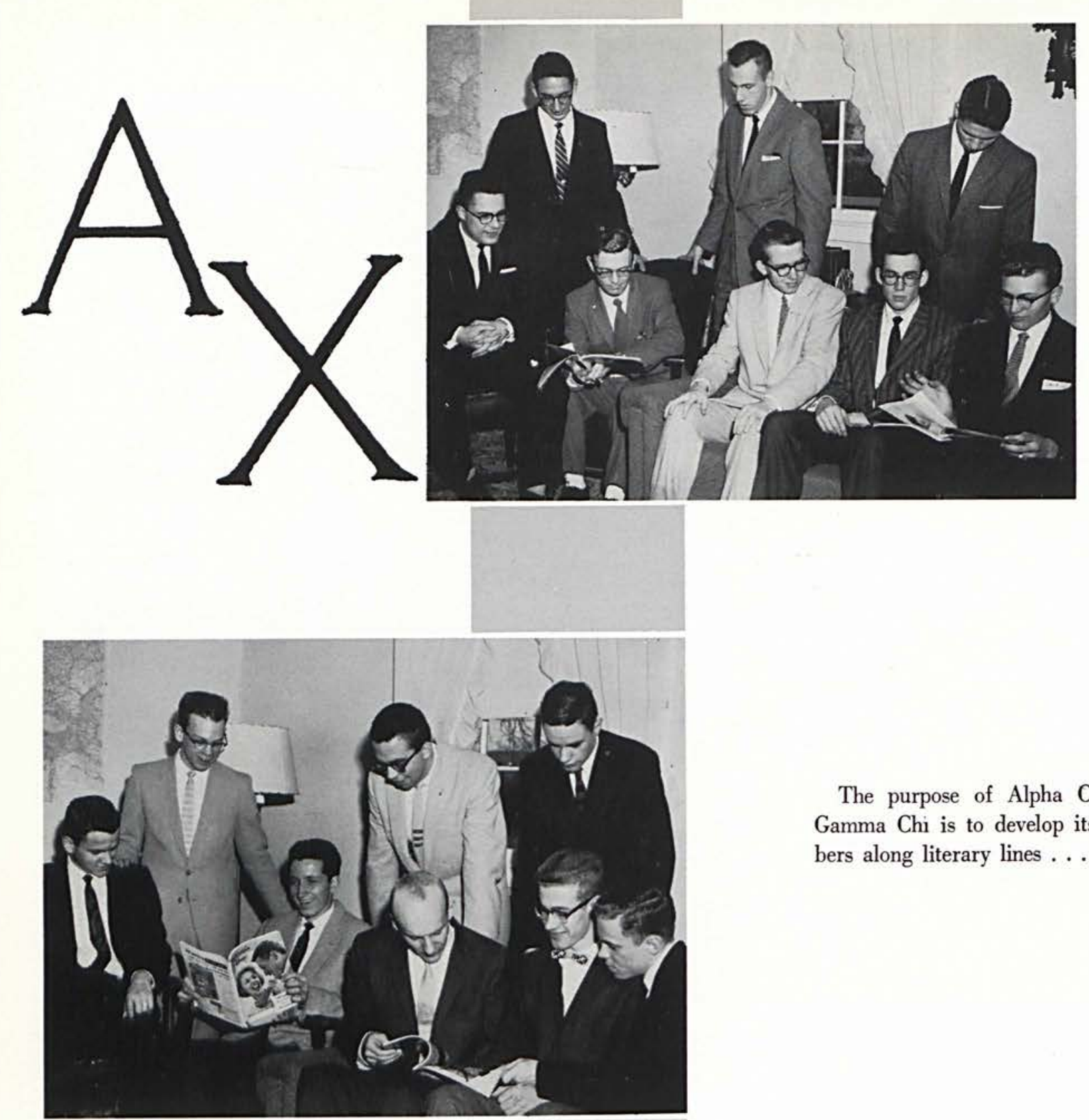

The purpose of Alpha Chi and Gamma Chi is to develop its members along literary lines...

President

Vice President

Secretary-Treasurer

Parliamentarian

Advisor
BOB HOWDER

DAVE THOMAS

TERRY GOODRICH

DAVE MATSON MR. HARMON BERGEN

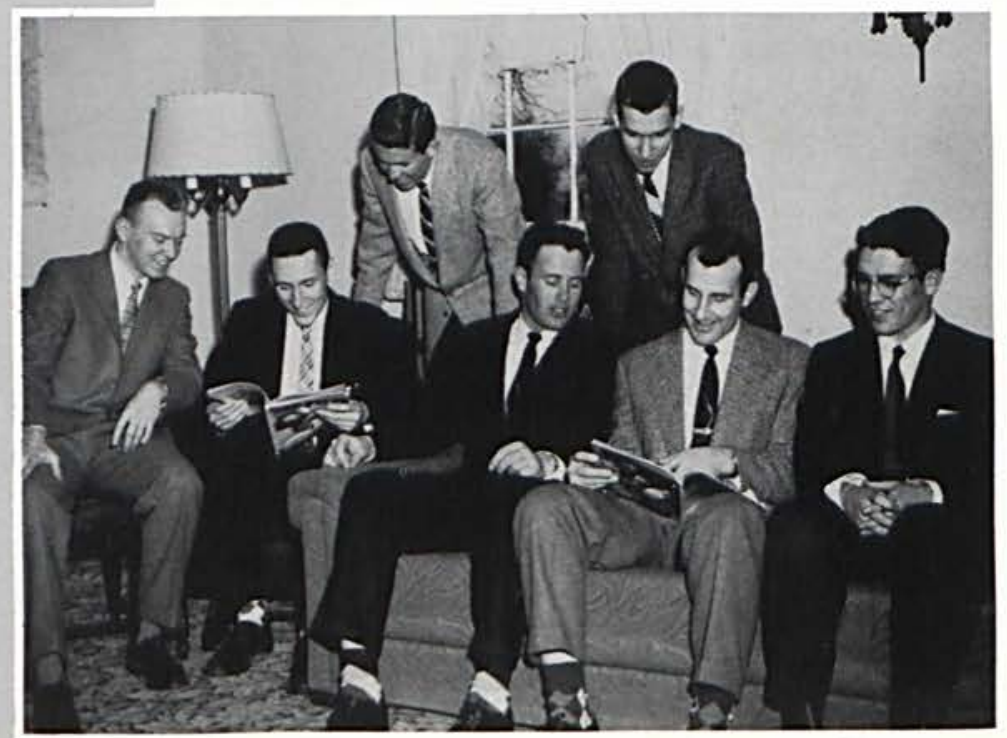



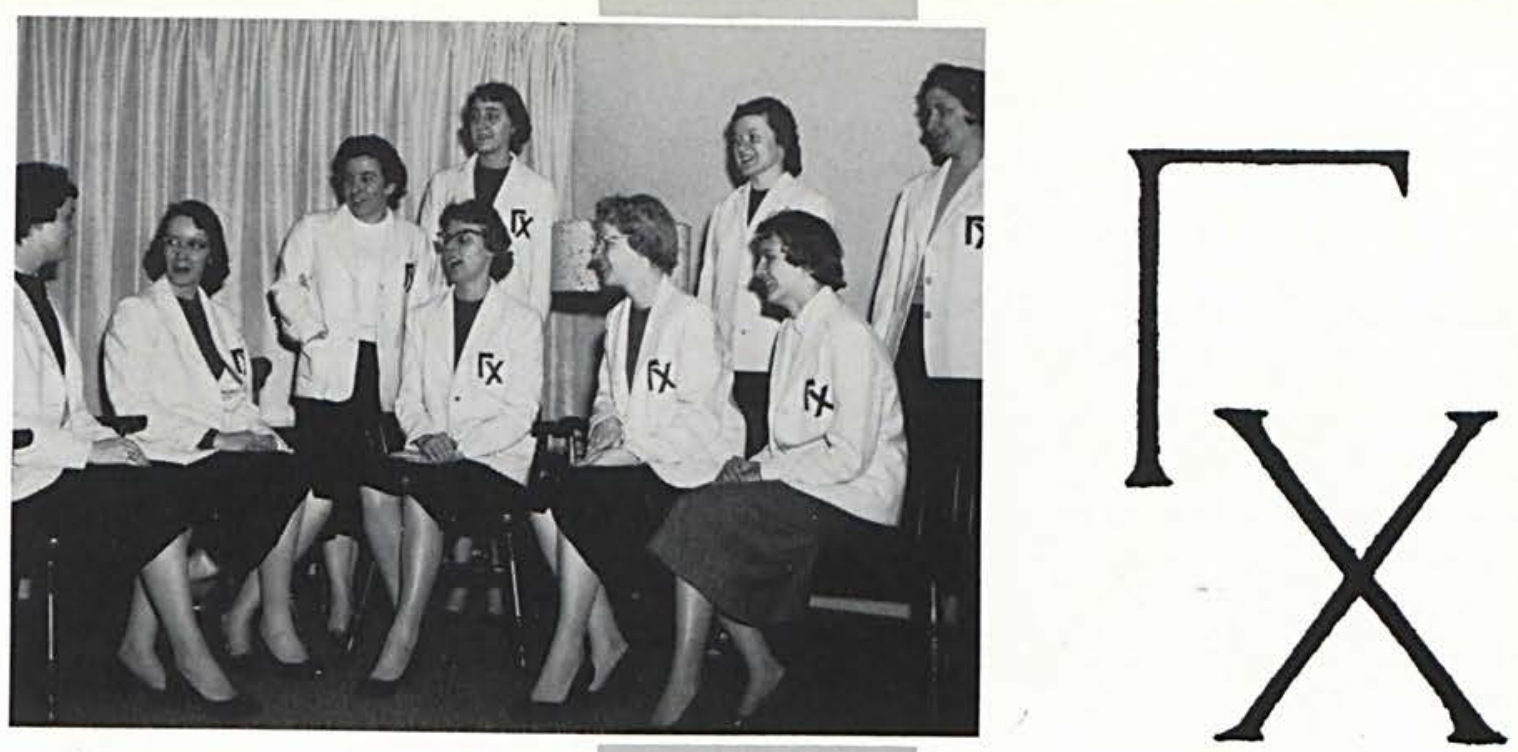

... to promote the spirit of refinement and social tastes; to develop poise, personality, and leadership at both formal and informal events.
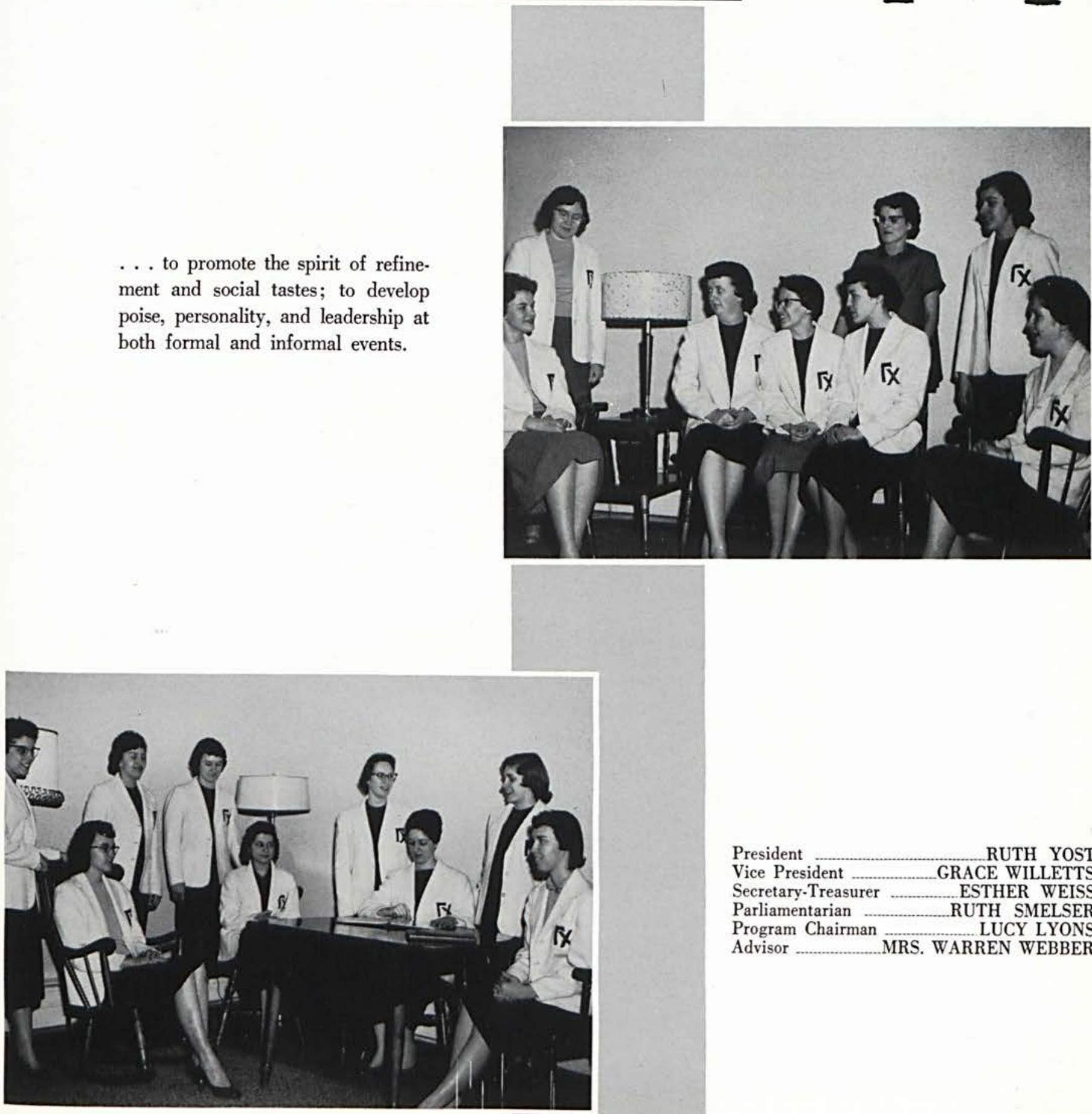

President RUTH YOST Vice President CE WILLETTS

Secretary-Treasure ESTHER WEISS Parliamentarian RUTH SMELSER

Program Chairman ………... LUCY LYONS Advisor MRS. WARREN WEBBER 


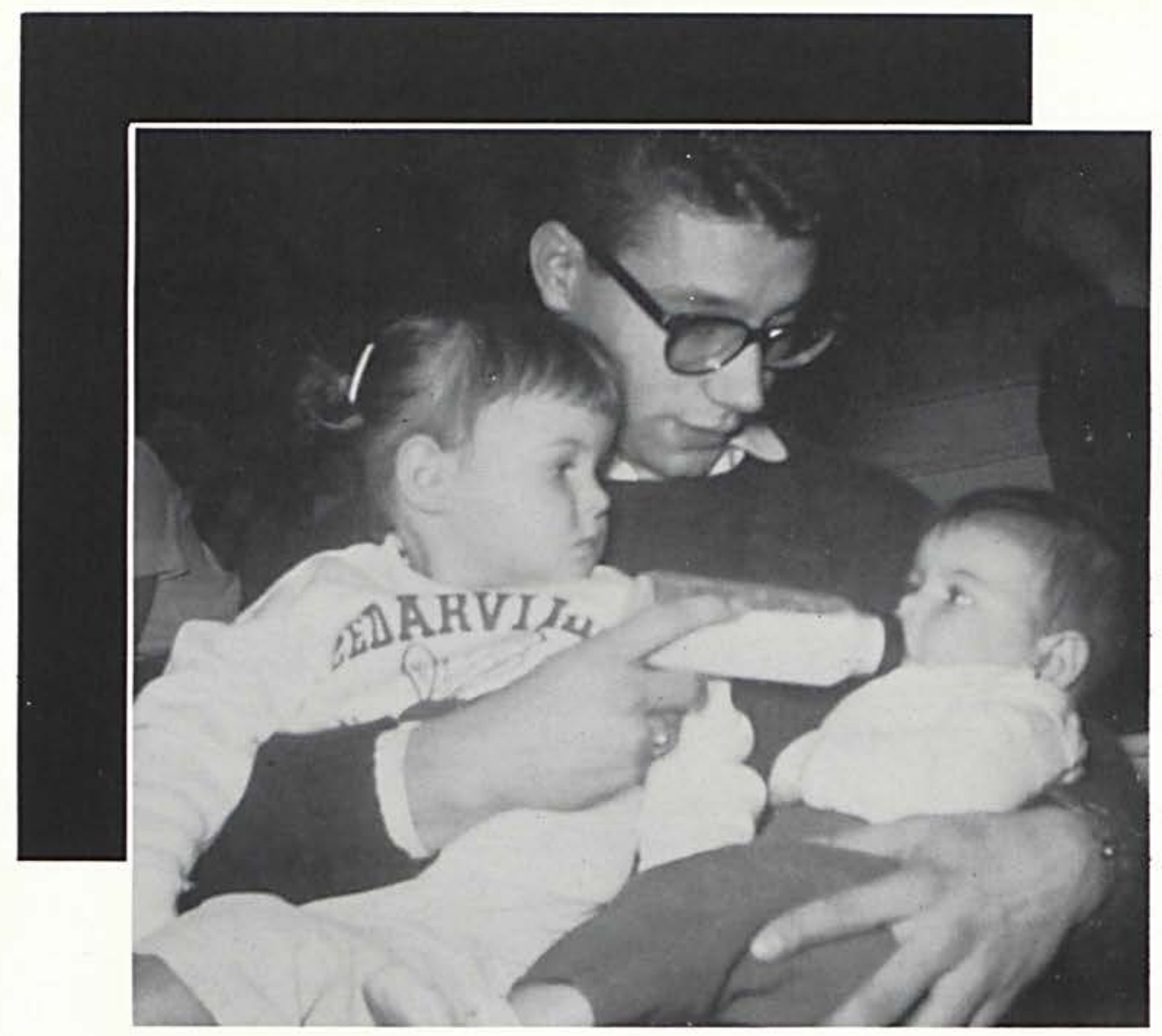

FIRST PRIZE: "Half time with 'Papa Steve.'” taken by Dolly Jesse

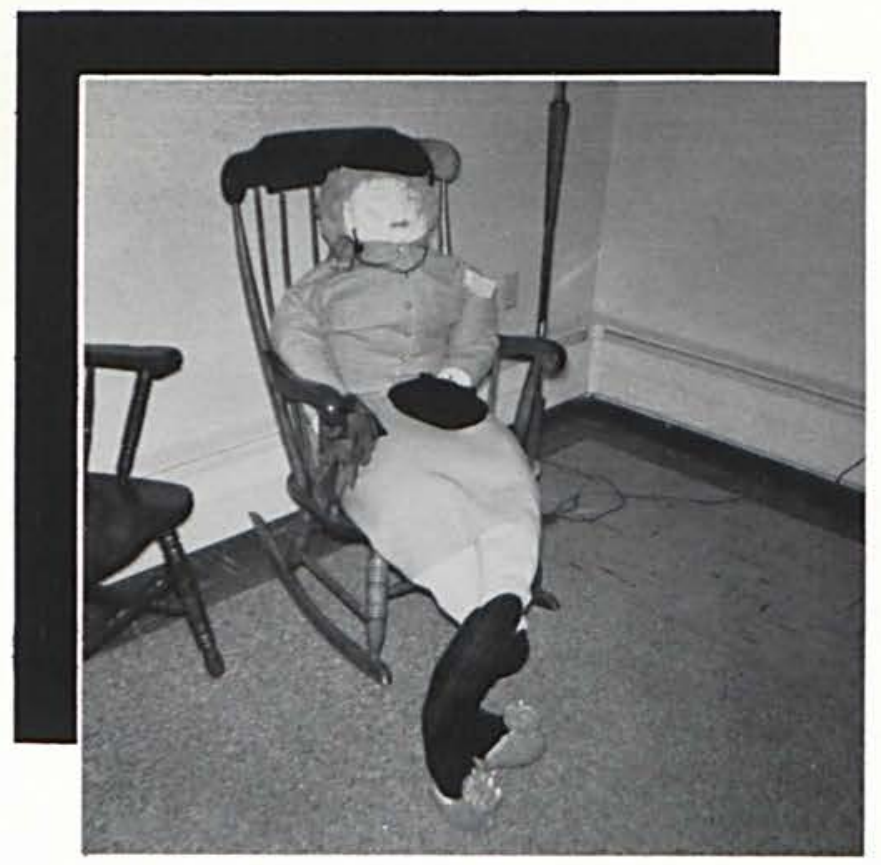

SECOND PRIZE: "I'm so-oo tired after that Smorgasbord." taken by Carmen Manchester.

\section{Photo Contest}




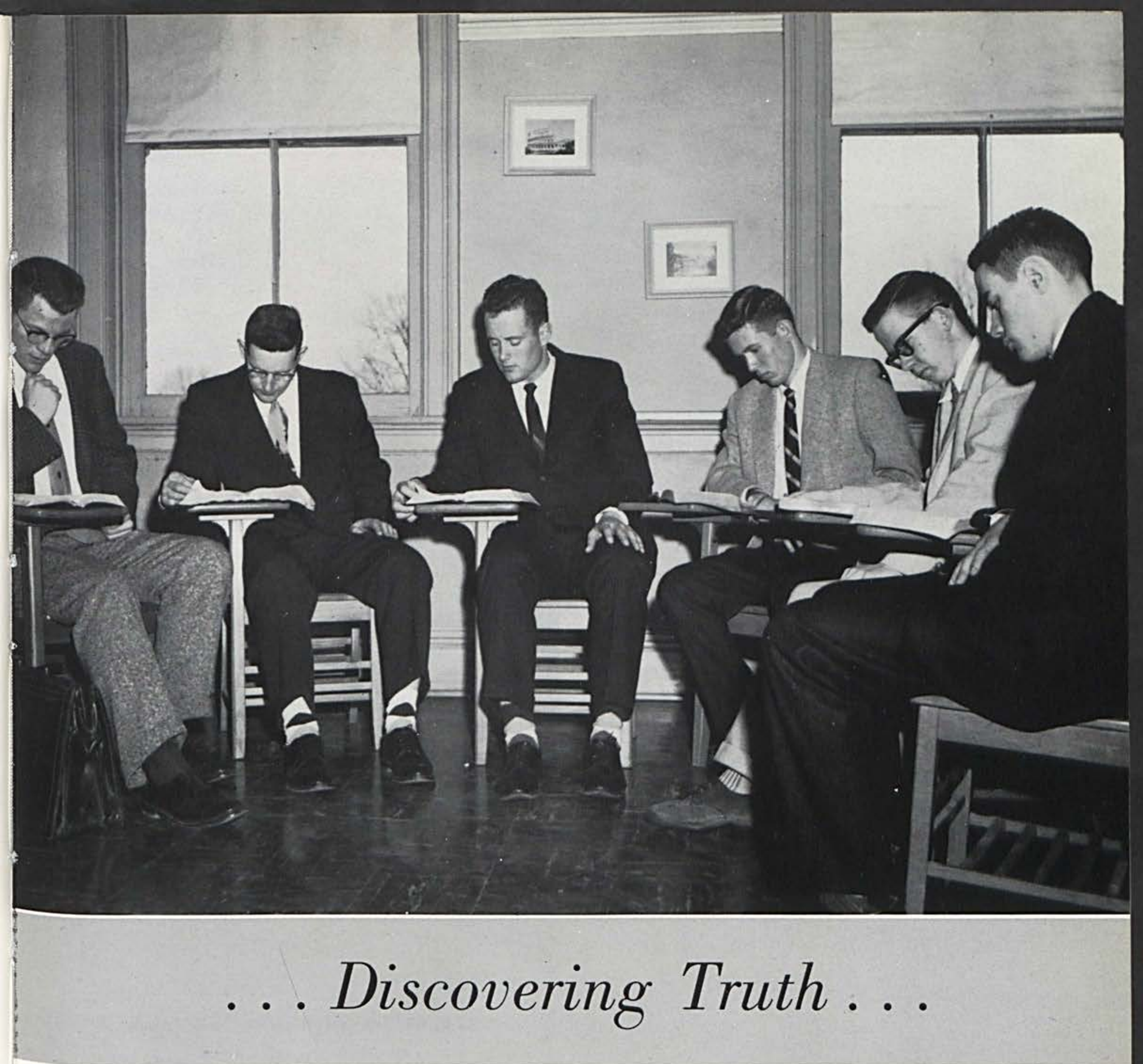




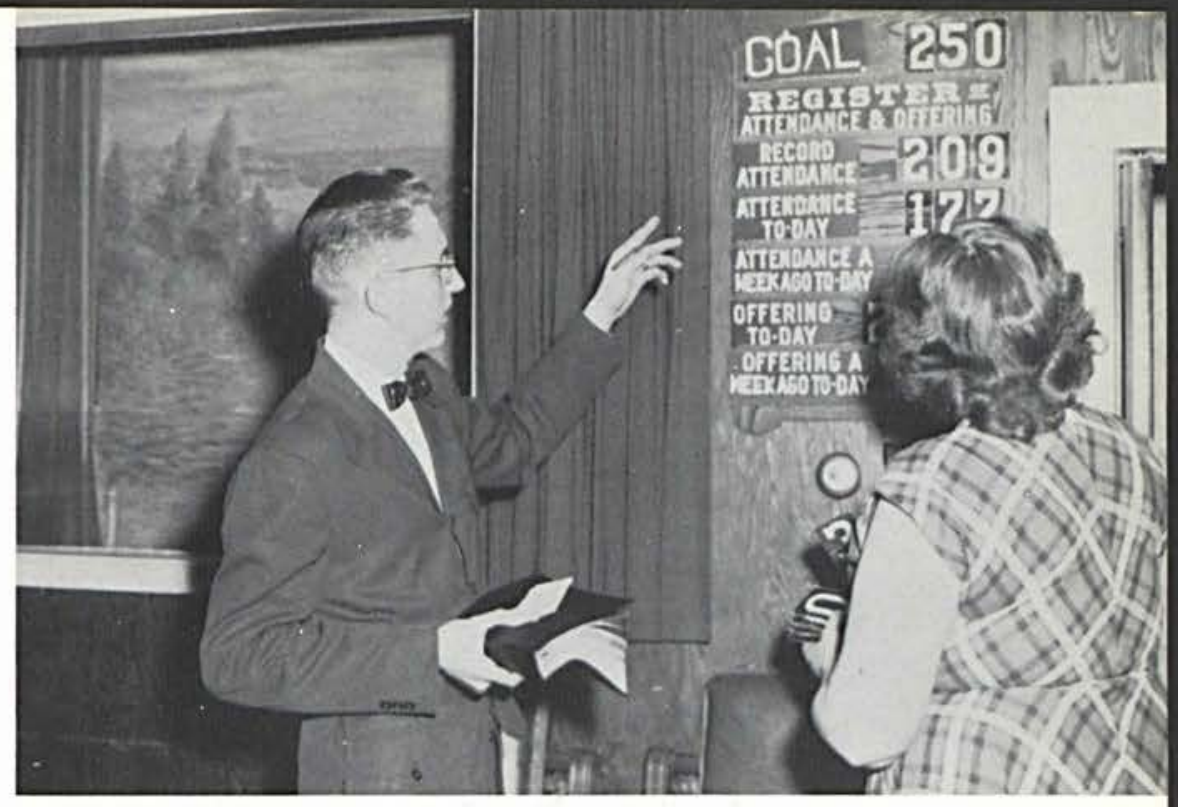

Ken Pierpont checks attendance as one of his many duties as Sunday School Superin. tendent.

\section{. . PREACH THE WORD, BE ZEALOUS IN SEASON AND OUT OF SEASON; . . .}

Don Sewell as pastor of the Williamsburg Baptist Church.
Keith Collett directing young people's choir.

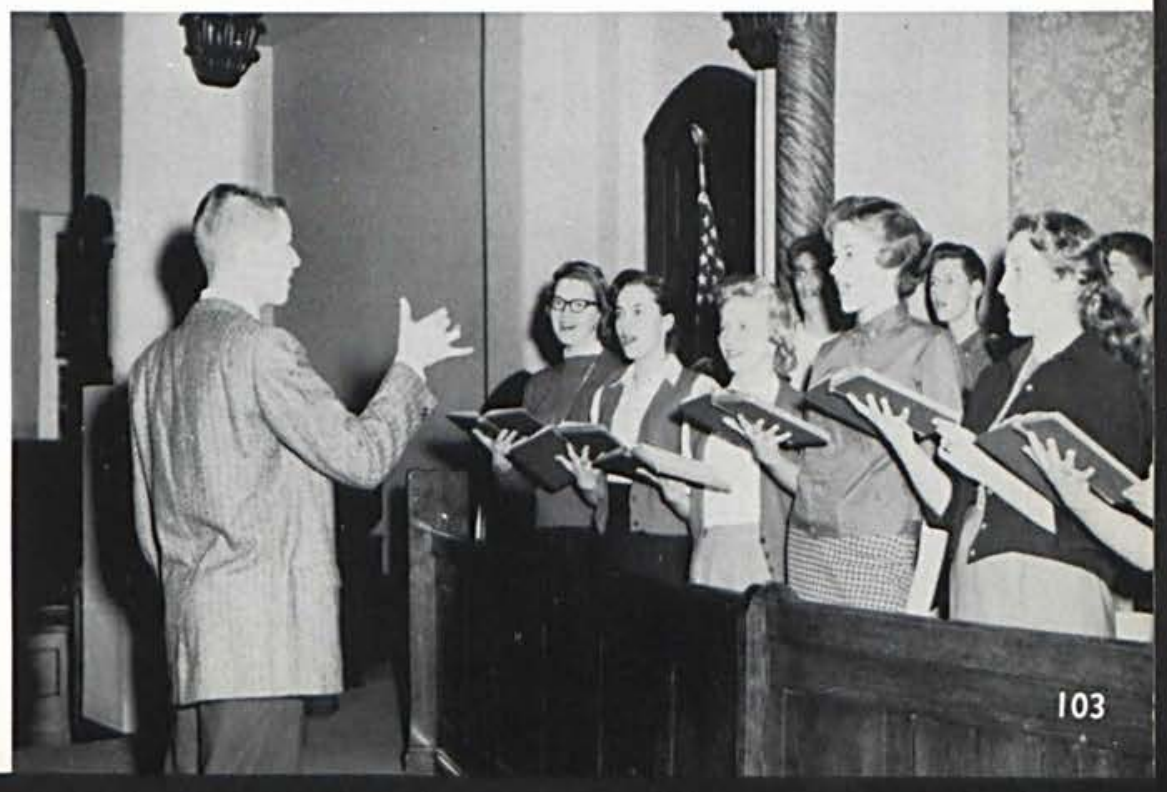




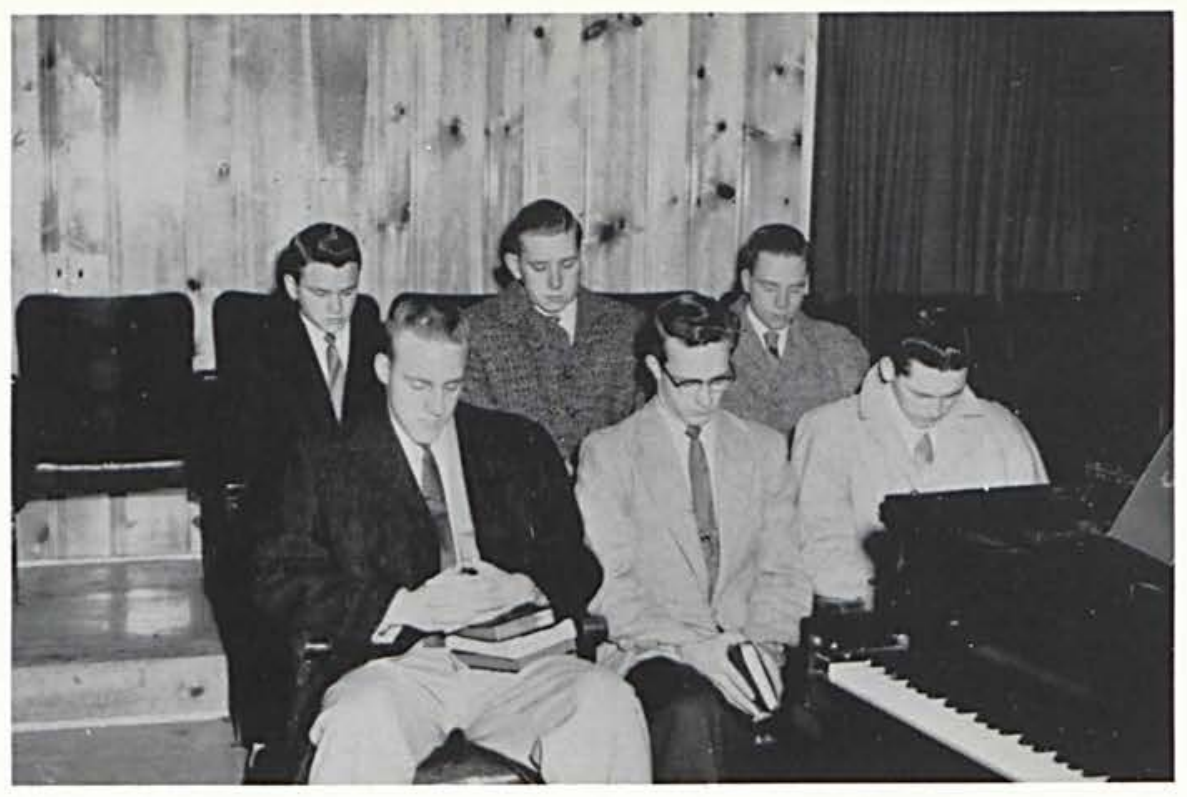

The Springfield Jail group meeting for prayer.

\section{... THEN WILL I TEACH TRANSGRESSORS THY WAYS; AND SINNERS SHALL BE CONVERTED UN- TO THEE . . .}

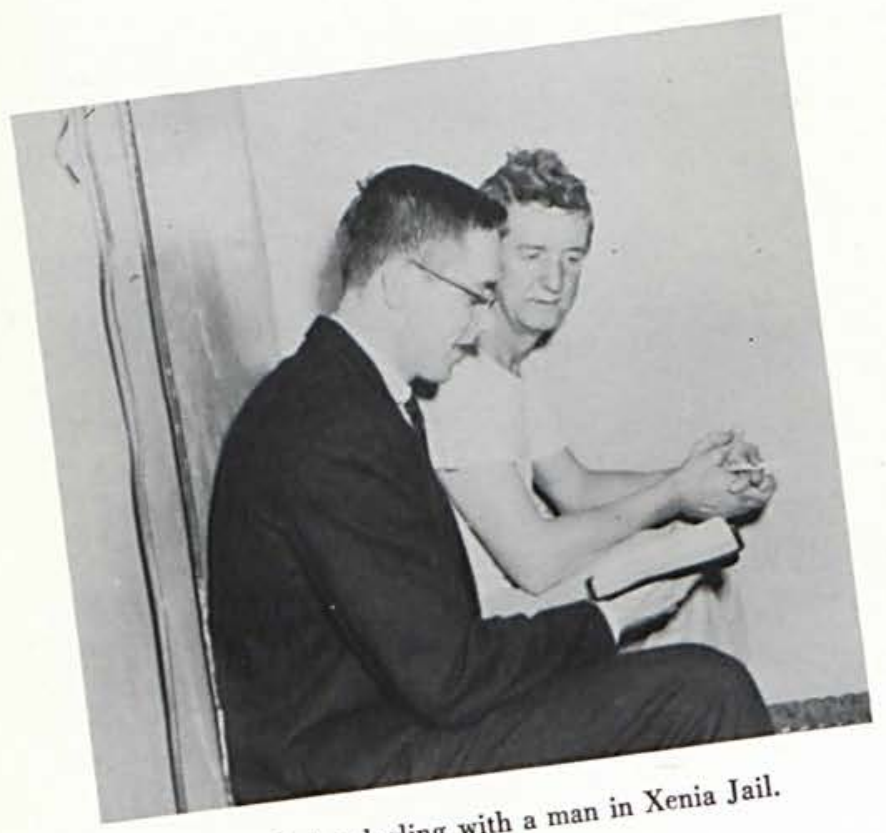

Terry Zerby dealing with a man in 


\section{Our Field Representative}

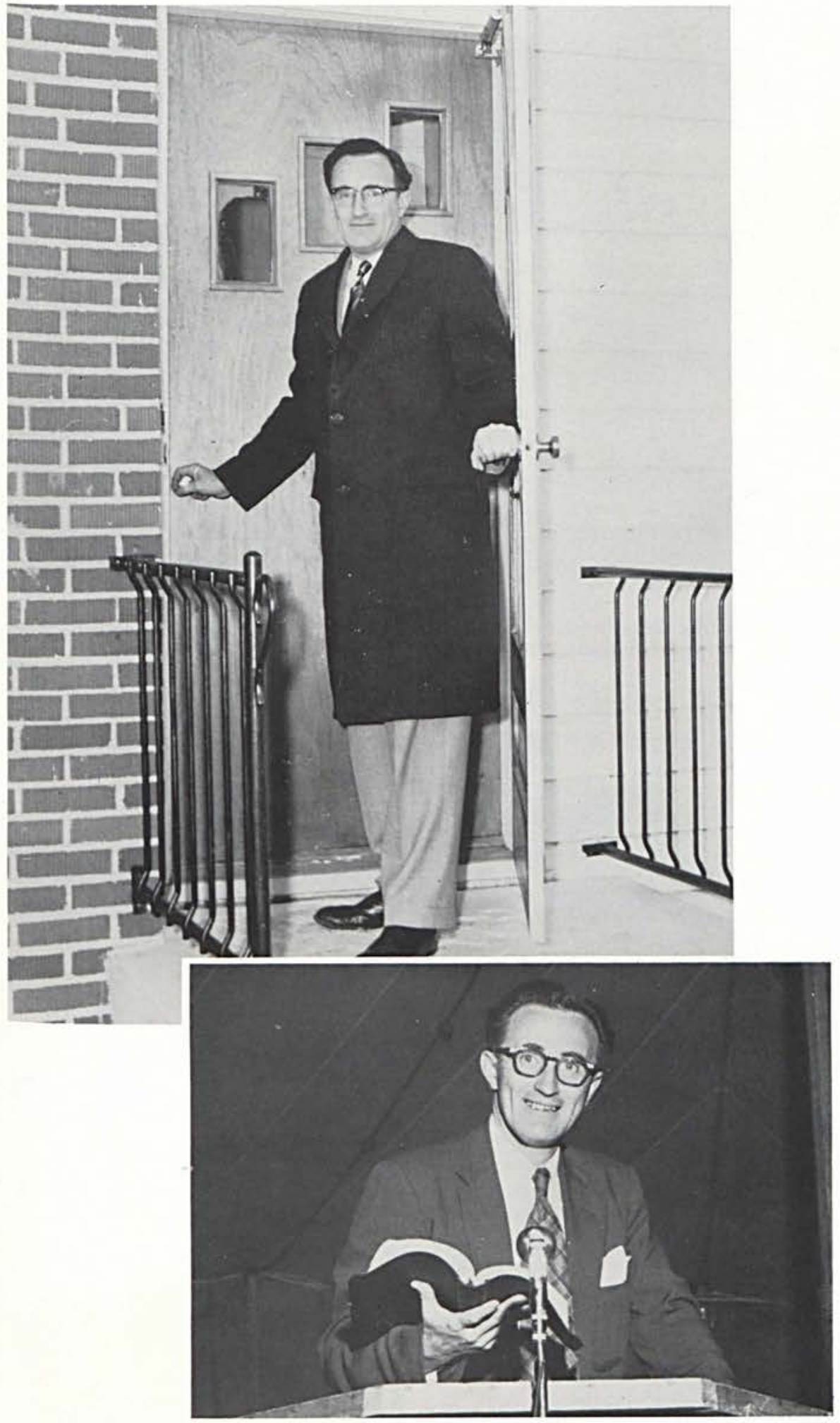

From Seattle, Washington, to Vestal, New York, Rev. Ernest A. Lockerbie has represented our college to the young people of our churches as he proclaimed the Gospel of Christ.

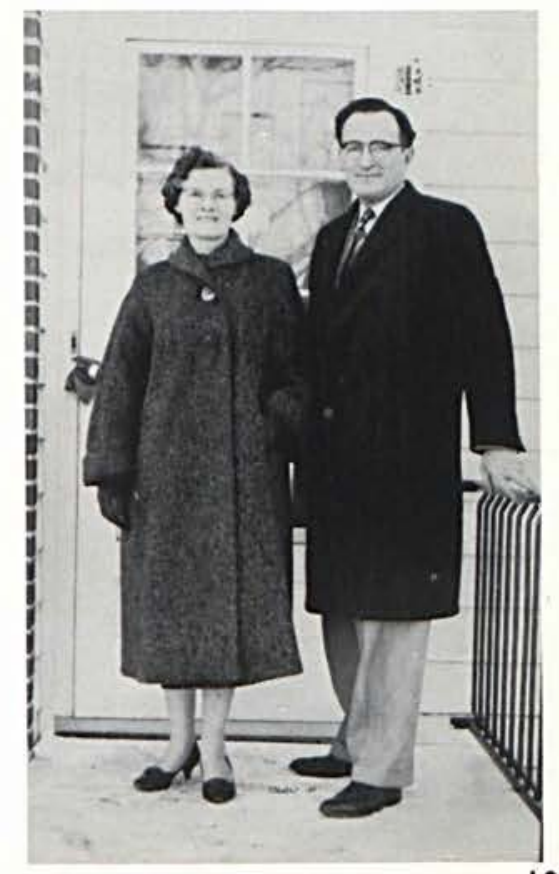




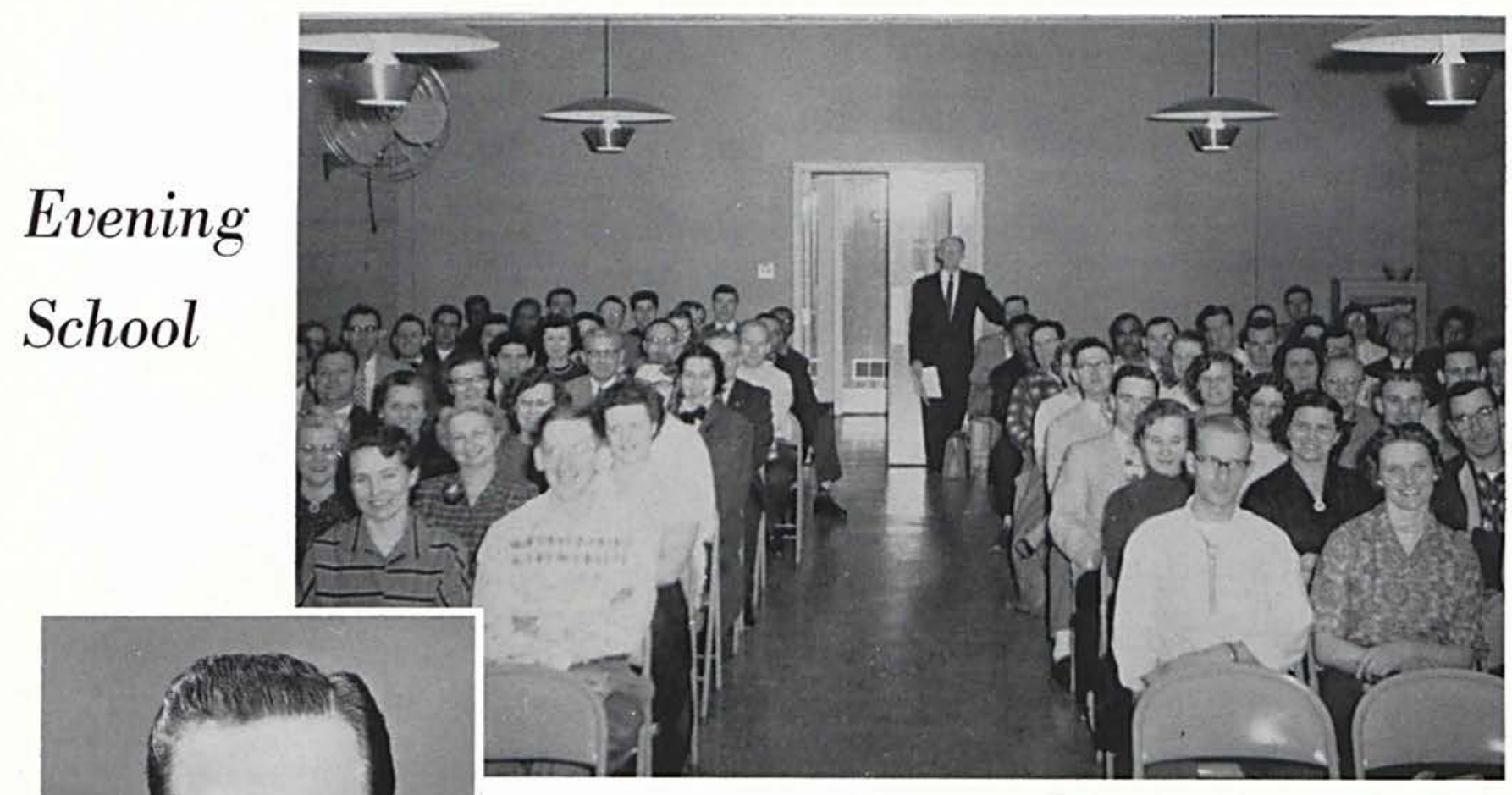

Evening school students meet at the Euclid-Nottingham Baptist Church, Cleveland, Ohio, for classes in Bible. During the first semester 103 students were enrolled.

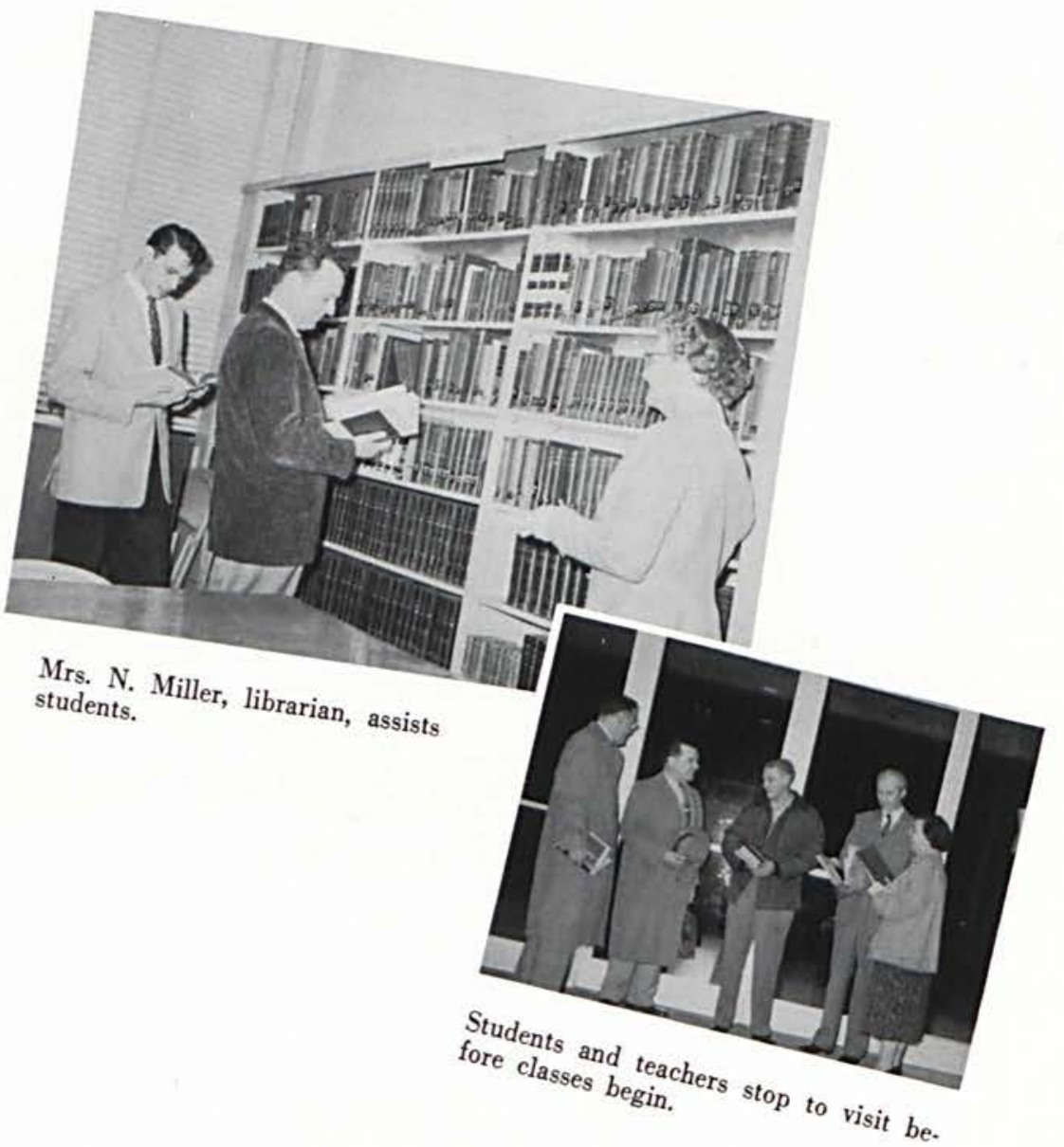




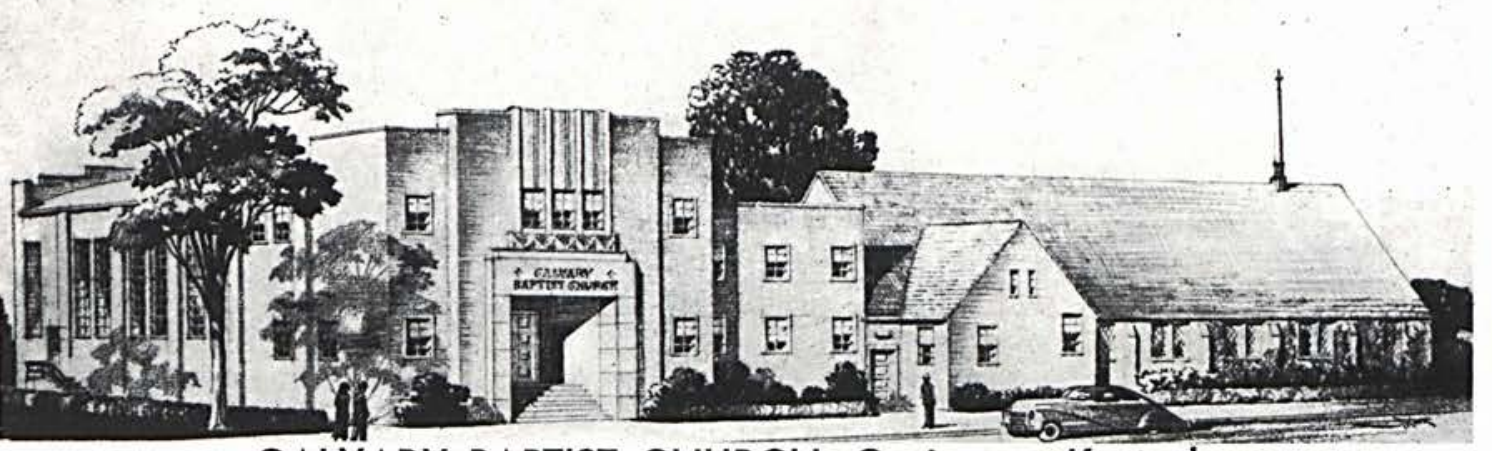

CALVARY BAPTIST CHURCH, Covington, Kentucky

D. B. EASTEP, Pastor
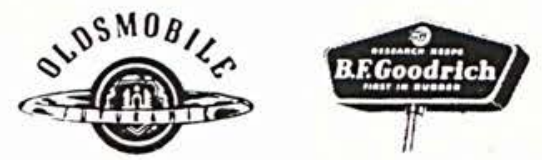

ADVERTISING
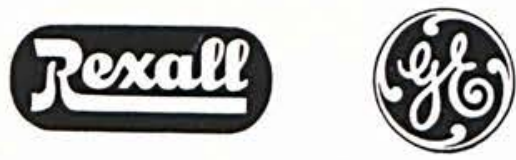

\section{CONGRATULATIONS}

to the

GRADUATING CLASS OF 1959

and to

CEDARVILLE for the important task excellently done of training its young men and women to serve God and their fellow men.

From the

GRAND RAPIDS "School of the Prophets," where the same spiritual emphasis is maintained and further training for Christian service is offered," that the man of God may be perfect, thoroughly furnished unto all good work."

\section{GRAND RAPIDS BAPTIST THEOLOGICAL SEMINARY}

8II Wealthy Street S. E.

GRAND RAPIDS 6, MICHIGAN

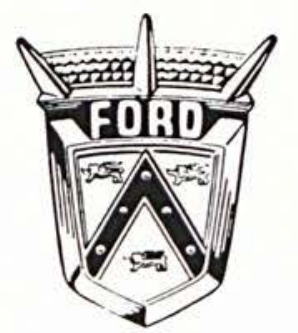

\section{Compliments of \\ ELLIS MOTOR SALES, INC.}

Ford Sales and Service

XENIA, OHIO

DR 2-6978

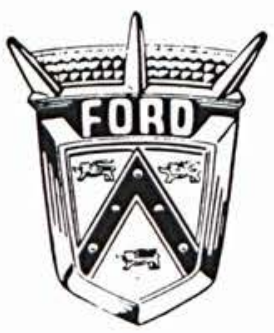




\section{CEDARVILLE}

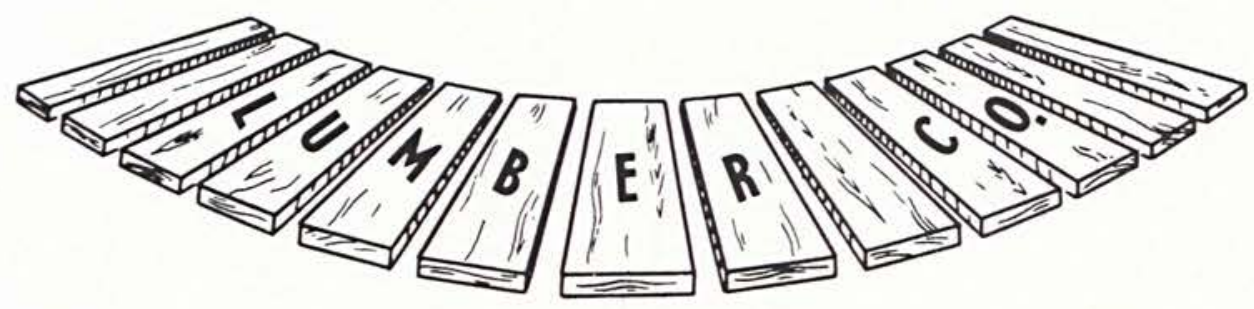

\section{MIAMI DEPOSIT BANK}

Complete Facilities

Ready to Serve You

CEDARVILLE, OHIO and

YELLOW SPRINGS, OHIO

Member of the FEDERAL DEPOSIT INSURANCE CORPORATION

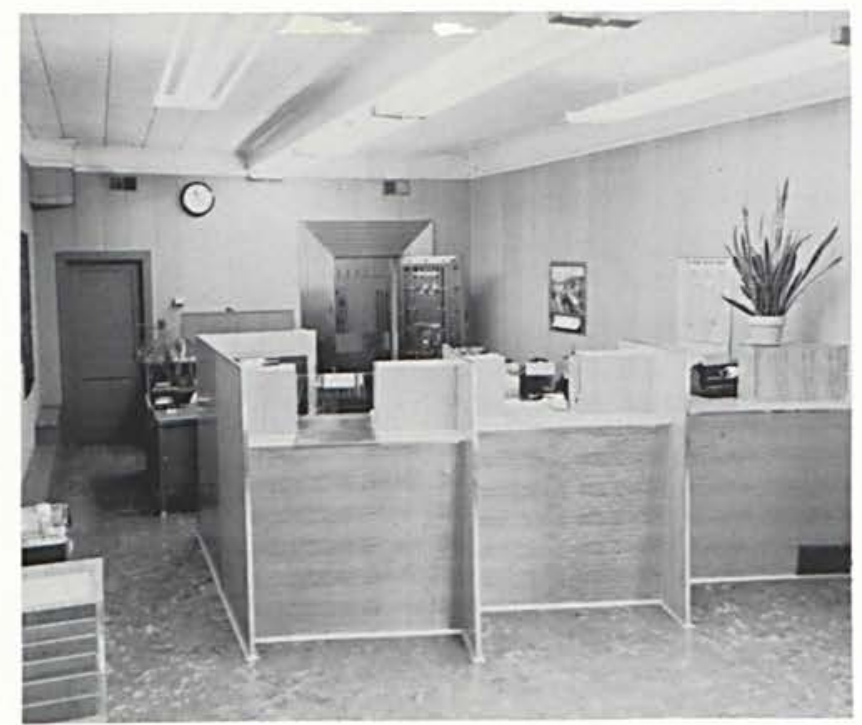

MUSIC

Is Our Business

BAND BOX MUSIC CENTER

3I West Main Street Xenia, Ohio
ELYRIA PLACEMENT SERVICE DYKE DIRECT MAIL SERVICE 30 Century Block ELYRIA, OHIO 


\section{FIRST REFORMED CHURCH}

Detroit and Church Streets

XENIA, OHIO

(Where the Bible Is Taught)

RUSSELL MAYER, Pastor

Independent, Fundamental, Premillennial

Congratulations

From the

FIRST BAPTIST CHURCH

of

Russell, lowa

ROBERT FELTZ, Pastor

Visit Us When You Visit lowa
Congratulations, Class of 1959 RIVERSIDE BAPTIST CHURCH

Corner South Jasper and East Cleveland Streets DECATUR, ILLINOIS

Pastor, GEORGE A. BATES

"To Him Who ever loves us and once for all released us from our sins by $\mathrm{His}$ blood, and has made us a kingdom of priests for His God and Father: to Him be glory forever. Amen."

Williams Translation Revelation I:5b and 6 


\title{
BEREA BAPTIST CHURCH
}

\author{
250 West Street \\ BEREA, OHIO
}

Bible School

9:30 A.M.

Morning Worship

10:45 A.M.

Bible Training Union

6:30 P.M.

II III

7:45 P.M. 7:45 P.M.

"That in all things $\mathrm{He}$ might have the pre-eminence"

EARL V. WILLETTS, Pastor

THE REYNOLDSBURG BIBLE MISSION BAPTIST CHURCH

\section{Pastor. LLEWELLYN THOMPSON}

Located on

South Taylor Road REYNOLDSBURG, OHIO

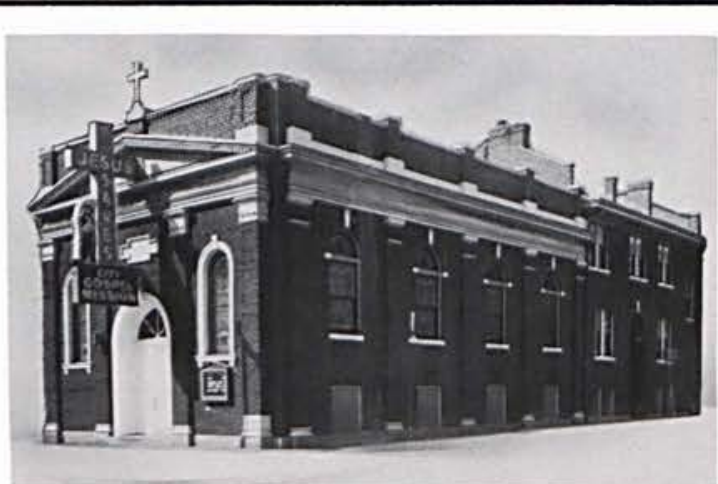

CITY GOSPEL MISSION

1419 Elm St., Cincinnati, Ohio

CH $\quad 1-5525$

Mrs. Marguerite Conn Superintendent

Ray Shepherd Religious Director Mrs. M. Von Briesen......Social Service Director Francis Francia Children's Worker

Open seven days a week, the mission ministers to the needy of the basin area with meals, clothing, lodgings, groceries, etc. 


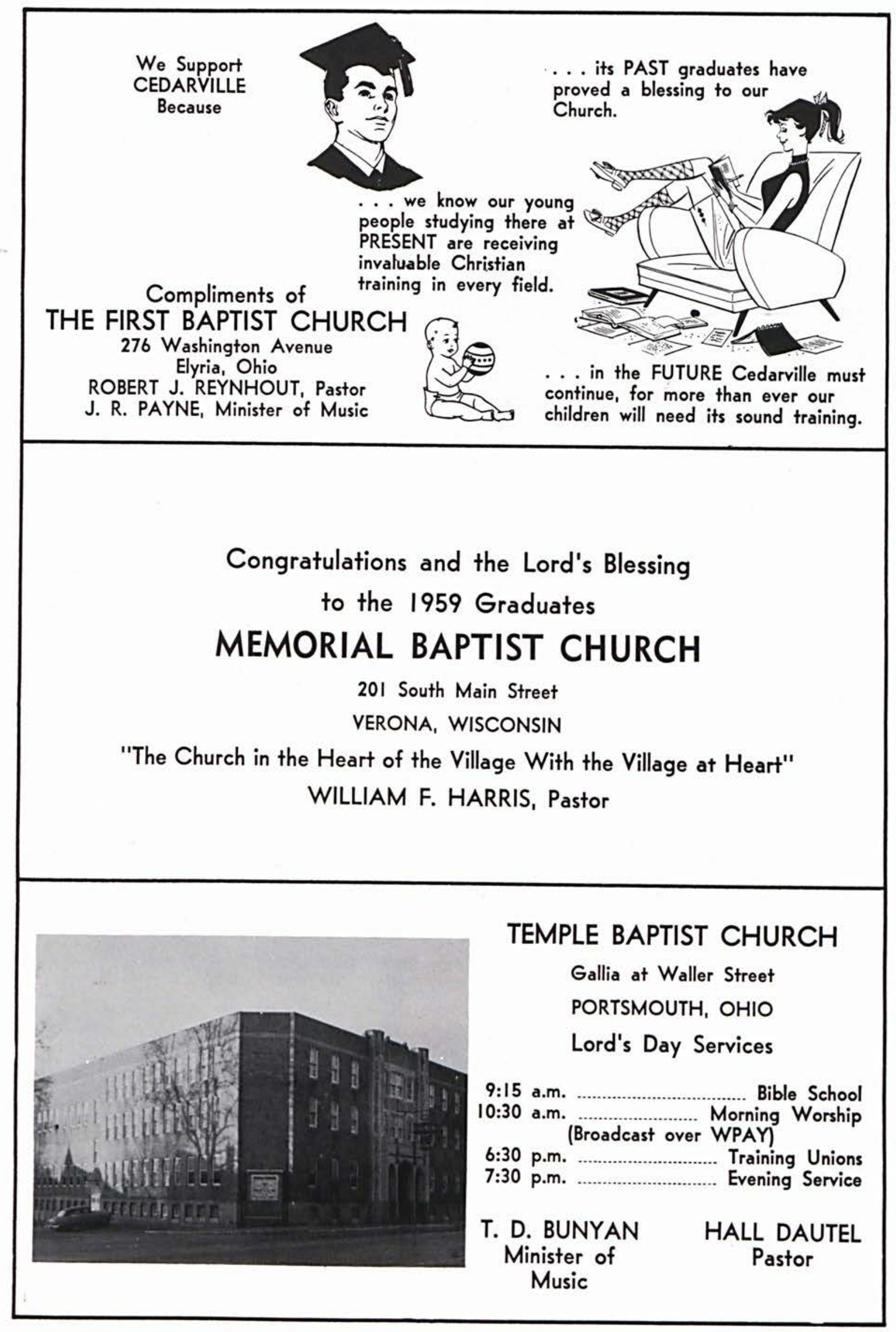




\title{
Holding Forth the Word of Life
}

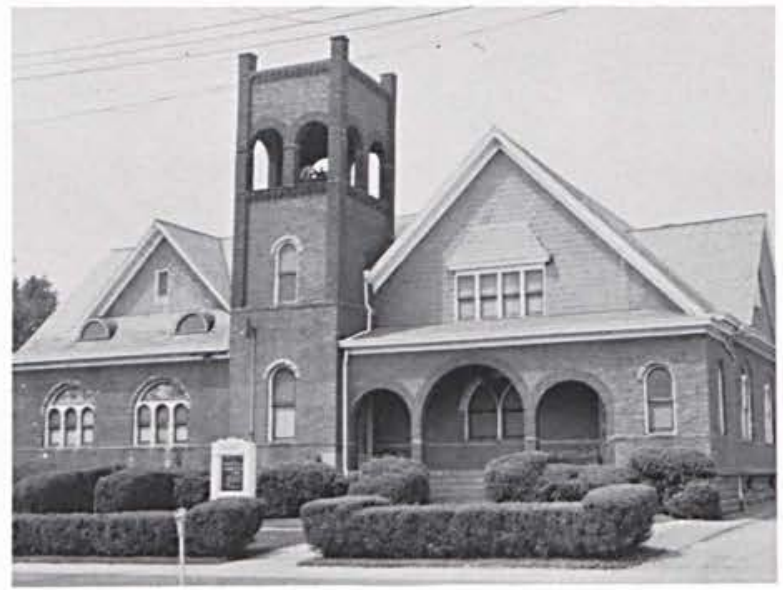

\section{FIRST BAPTIST CHURCH}

\author{
MEDINA, OHIO
}

Just Off the Square on

West Liberty

In the Heart of Medina, With Medina on Its Heart

R. KENNETH SMELSER, Pastor

Congratulations to the Senior Class

From the

\section{EUCLID-NOTTINGHAM BAPTIST CHURCH}

I890| Lake Shore Boulevard

Euclid 19, Ohio

REV. ALLAN E. LEWIS, Pastor

FIRST BAPTIST CHURCH

\section{II8 West Walnut Street Galion, Ohio}

SUNDAY

Bible School

Worship Service

Baptist Youth Fellowship

Gospel Service 9:30 a.m. 10:30 a.m. $6: 30$ p.m. 7:45 p.m. WEDNESDAY

Prayer Meeting and Bible Study 7:30 p.m.

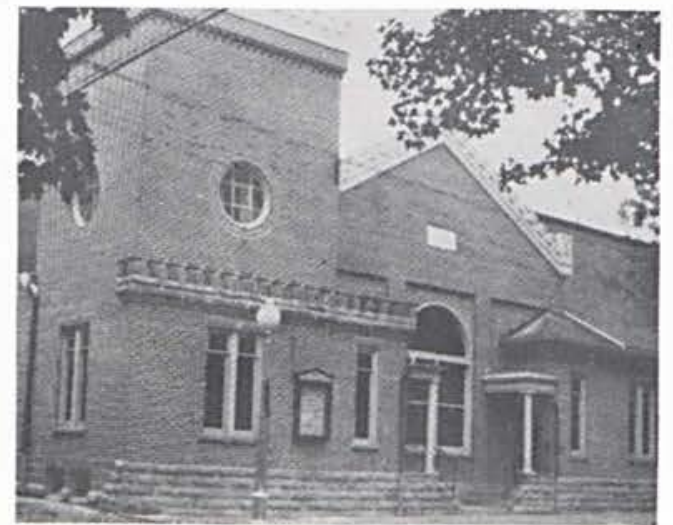

REV. WILFRED BOOTH, Pastor 


\title{
Best Wishes HALE DRUGS
}

\author{
Your Walgreen Agency
}

Friendly, Personalized Service

Prescription Specialists

TELEPHONE TU 2-3113

MAIN STREET

SYLVANIA, OHIO

\section{JIM BURKS \\ BARBER SHOP}

70 Main Street

CEDARVILLE, OHIO

Special Rates to College Students

\section{LANG'S}

CHEVROLET OLDSMOBILE

$$
\text { Xenia, Ohio }
$$

Fairborn, Ohio

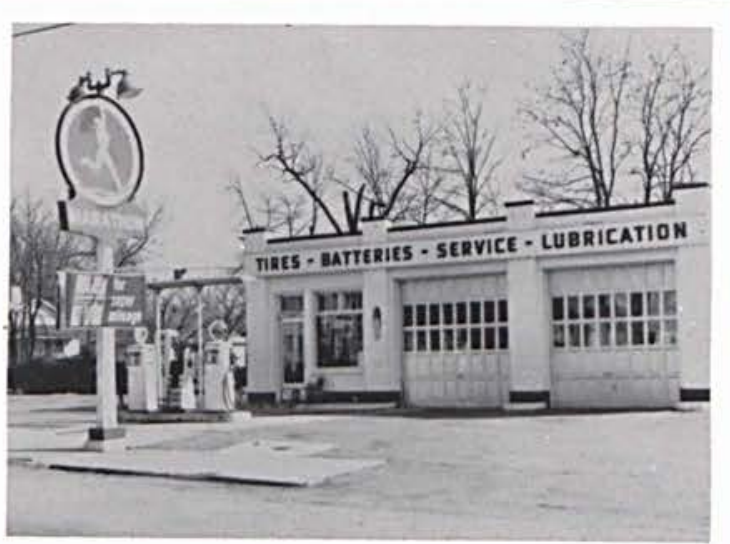

Marathon

HAGLER

SERVICE STATION

Phone SO 6-0110

CEDARVILLE, OHIO 


\section{Compliments of}

\section{MORRIS BEAN}

AND COMPANY

YELLOW SPRINGS, OHIO

CEDARVILLE, OHIO

Compliments

of

STOKES

\section{MOTOR COMPANY}

South Main Street

CEDARVILLE, OHIO

Phone SO 6-4021

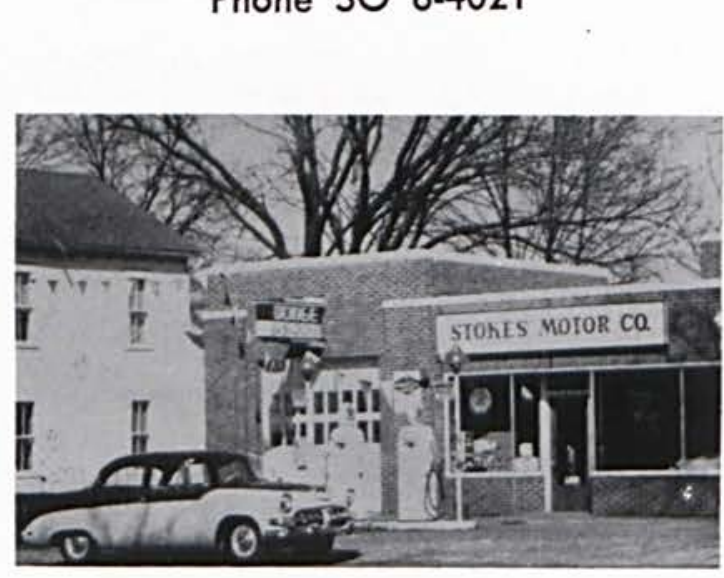

\section{Compliments of CEDARVILLE HARDWARE}

Progressive Hardware Service

CEDARVILLE, OHIO

PHONE SO 6-1941

Your GE Appliance Dealer

RANDALL

\&

STORMONT

INC.

Phone SO 6-1031

CEDARVILLE $\mathrm{OHIO}$

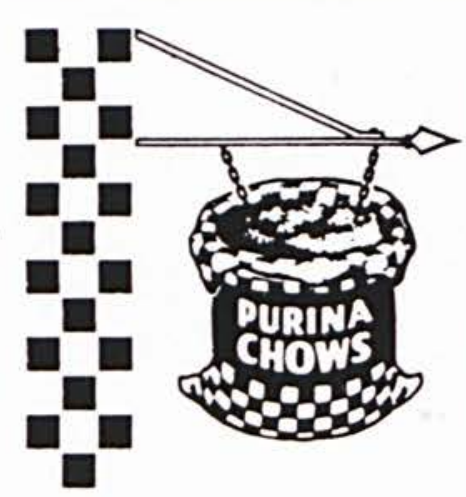




\begin{tabular}{|c|c|}
\hline 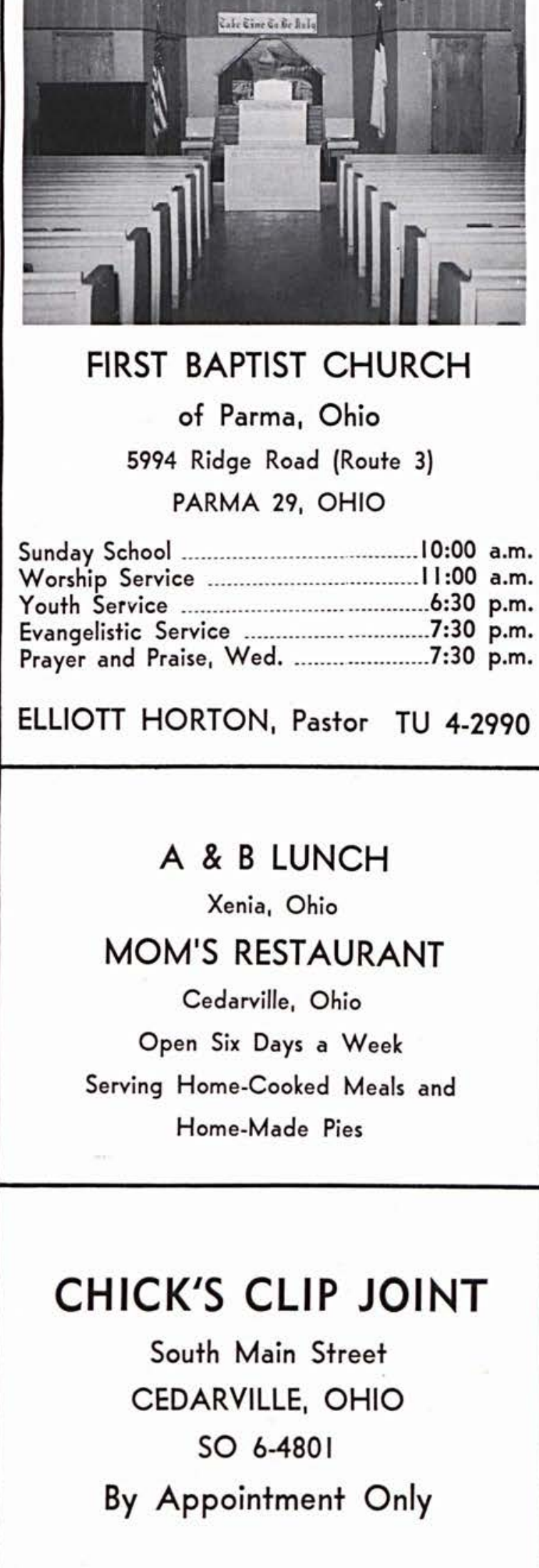 & 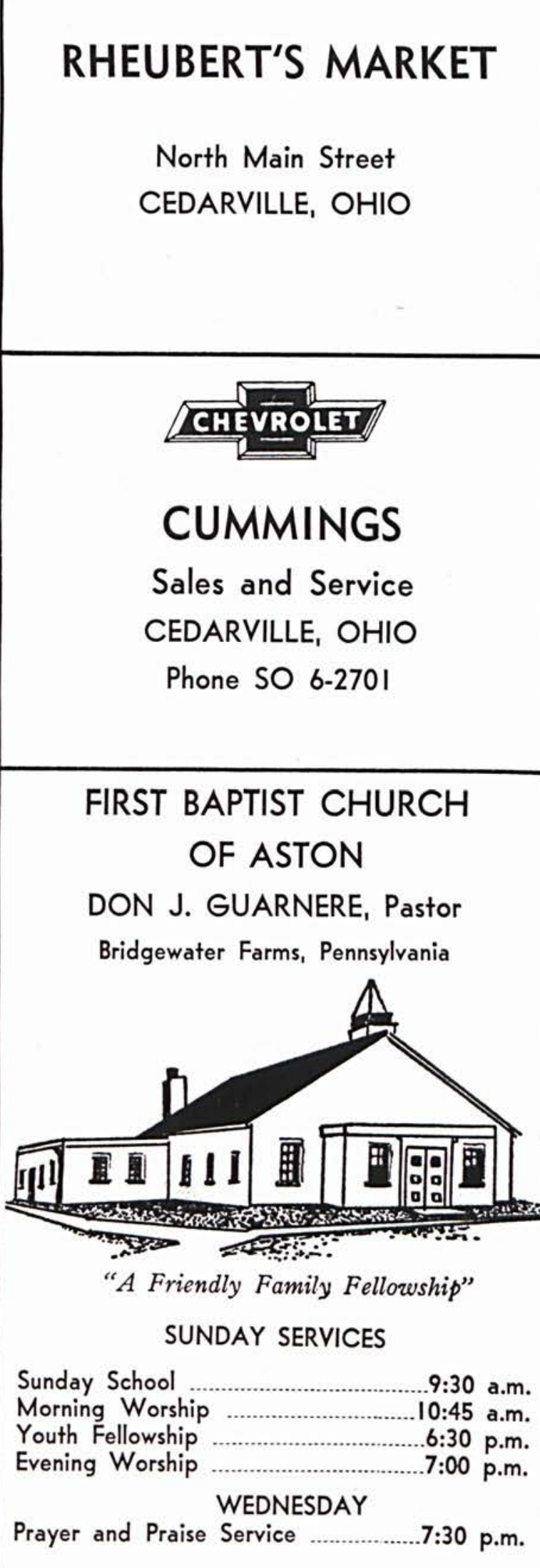 \\
\hline
\end{tabular}




\section{JAMES CUT-RATE}

The Rexall Store

Phone SO 6-177I

CEDARVILLE, OHIO

\section{CHAPLIN CLEANERS}

Laundry Service

Cleaning

Shoe Repair

Pressing

CEDARVILLE, OHIO

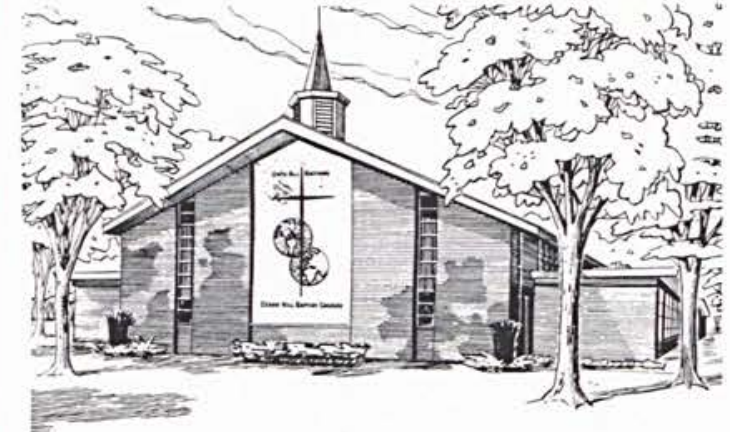

CEDAR HILL BAPTIST CHURCH

John G. Balyo, pastor

Earl Leiby, associate pastor

12601 Cedar Road, Cleveland Heights 6, O.

\section{HARNER'S \\ CEDARVILLE MARKET}

Meats

Produce Groceries

Phone SO 6-1201

CEDARVILLE, OHIO
CONGRATULATIONS, CEDARVILLE on Another

Miracle Year!!!

"God Answers Prayer!" $* * * * * *$

FELLOWSHIP OF BAPTISTS FOR HOME MISSIONS ELYRIA, OHIO *******

Re-Churching America With True Baptist Churches 




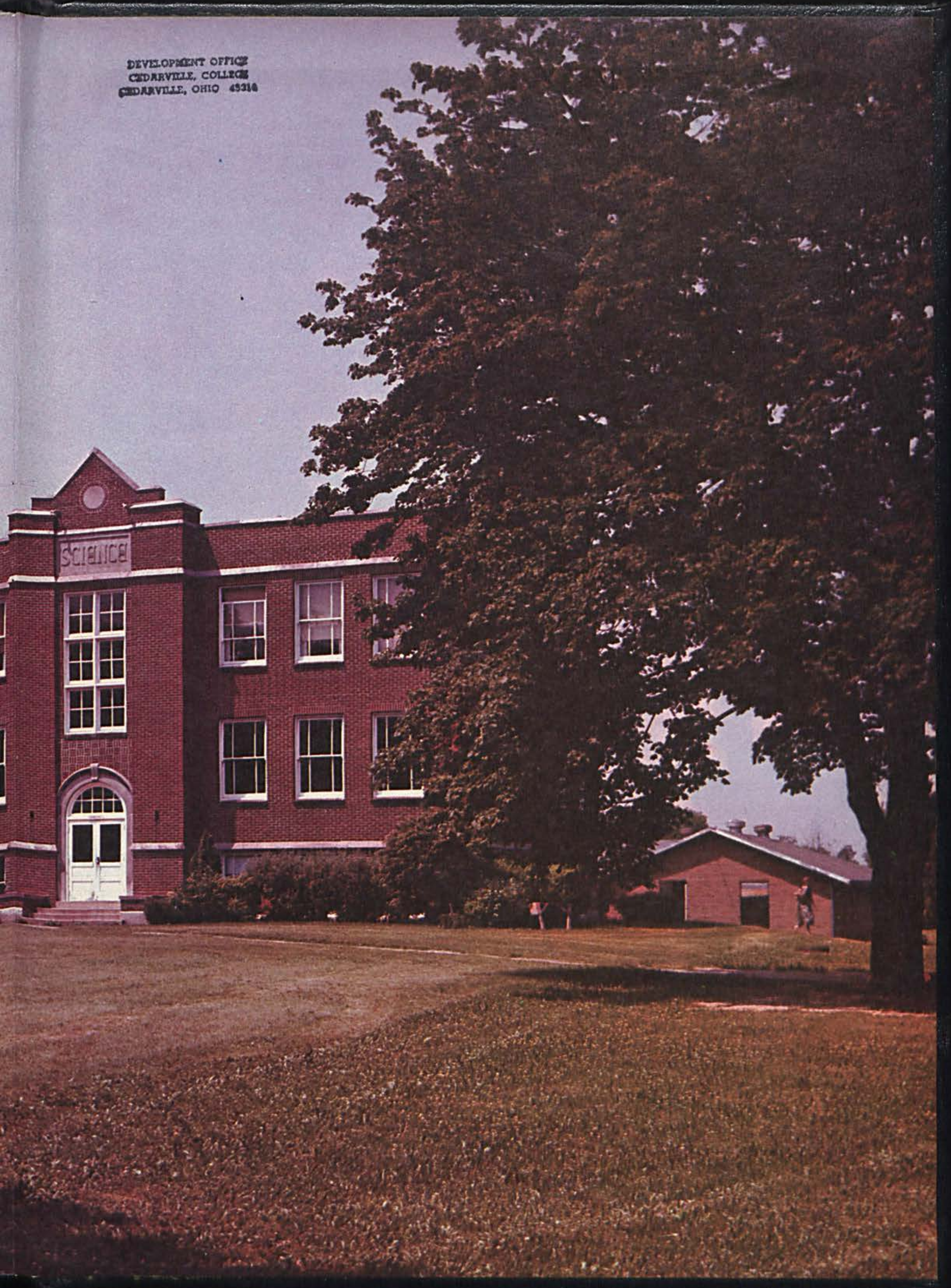


